\title{
Quaderni Veneti
}

Nuova serie digitale

Vol. 9

Dicembre 2020

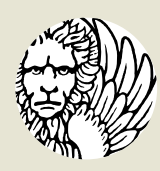

Edizioni

Ca'Foscari 



\section{Quaderni Veneti}

Direttore

Tiziano Zanato

Edizioni Ca' Foscari - Digital Publishing Fondazione Università Ca' Foscari Dorsoduro 3246, 30123 Venezia URL http://edizionicafoscari.unive.it/it/edizioni/riviste/quaderniveneti/ 


\title{
Quaderni Veneti
}

\author{
Rivista annuale
}

Direzione scientifica Tiziano Zanato (Università Ca’ Foscari Venezia, Italia)

Comitato scientifico Rossend Arqués Corominas (Universitat Autònoma de Barcelona, España) Daniele Baglioni (Università Ca’ Foscari Venezia, Italia) Francesco Bruni (Università Ca' Foscari Venezia, Italia) Eugenio Burgio (Università Ca' Foscari Venezia, Italia) Michele Cortelazzo (Università degli Studi di Padova, Italia) Elisa Curti (Università Ca' Foscari Venezia, Italia) Luca D’Onghia (Scuola Normale Superiore di Pisa, Italia) Riccardo Drusi (Università Ca' Foscari Venezia, Italia) Andrea Fabiano (Université Paris-Sorbonne, France) Angela Fabris (Alpen-Adria-UniversitätKlagenfurt, Österreich) Carla Marcato (Università degli Studi di Udine, Italia) Anna Rinaldin (Università Telematica Pegaso, Italia) Franco Tomasi (Università degli Studi di Padova, Italia) Lorenzo Tomasin (Universitéde Lausanne, Suisse) Pier Mario Vescovo (Università Ca' FoscariVenezia, Italia) Nikola Vuletić (Università di Zadar (Zara), Croazia)

Segreteria di redazione Samuela Simion (Università Ca' Foscari Venezia, Italia)

Direttore responsabile Michela Rusi (Università Ca' Foscari Venezia, Italia)

\section{Direzione e redazione}

Università Ca' Foscari Venezia, Dipartimento di Studi Umanistici

Dorsoduro 3246, 30123 Venezia, Italia

Editore Edizioni Ca' Foscari - Digital Publishing | Fondazione Università Ca' Foscari | Dorsoduro 3246, 30123 Venezia, Italia | ecf@unive.it

C 2022 Università Ca' Foscari Venezia

(C) 2022 Edizioni Ca' Foscari - Digital Publishing per la presente edizione

\section{(ㄷ)(1)}

Quest'opera è distribuita con Licenza Creative Commons Attribuzione 4.0 Internazionale This work is licensed under a Creative Commons Attribution 4.0 International License

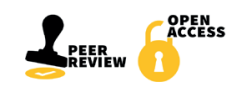

Certificazionescientifica delle Opere pubblicate da Edizioni Ca' Foscari - Digital Publishing: tutti isagg pubblicati hanno ottenuto il parere favorevole da parte di valutatori esperti della materia, attraverso un processo di revisione anonima sotto la responsabilità del Comitato scientifico della rivista. La valutazione è stata condotta in aderenza ai criteri scientifici ed editoriali di Edizioni Ca’ Foscari.

Scientific certification of the works published by Edizioni Ca' Foscari - Digital Publishing: all essays published in this volume have received a favourable opinion by subject-matter experts, through an anonymous peer review process under the responsibility of the Scientific Committee of the journal. The evaluations were conducted in adherence to the scientific and editorial criteria established by Edizioni Ca' Foscari. 
Quaderni Veneti

e-ISSN 1724-188X

Vol. 9 - Dicembre 2020

\section{Sommario}

ARTICOLI

Il volgare trentino in una tariffa rivana del 1409

Stefano Cristelli, Mario Wild

La biblioteca di una filantropa

Libri e letture di Stéphanie Omboni (1839-1917)

Vincenzo Vozza

Rapporti culturali nella tradizione poetica del Novecento dalle lettere di Ernesto Calzavara

Anna Rinaldin

Omaggio ad Andrea Zanzotto

Patrizia Valduga e Riccardo Held

Silvana Tamiozzo Goldmann

NOTE E RECENSIONI

Mario Isnenghi

Vite vissute e no. I luoghi della mia memoria

Francesca Bisutti 



\section{Articoli}





\title{
Quaderni Veneti
}

Vol. 9 - Dicembre 2020

\section{Il volgare trentino in una tariffa rivana del 1409}

\author{
Stefano Cristelli \\ Universität Zürich, Schweiz \\ Mario Wild \\ Universität Zürich, Schweiz
}

\begin{abstract}
This article analyses the language of an early fifteenth-century manuscript concerning the muta (excise taxes) of Riva del Garda (Trentino, Italy). The linguistic interest of said document, written mainly in Latin, lies in the number of vernacular terms and their nature (mostly mercantile goods). The authors provide a partial edition of the source, which includes also a series of business notes, a linguistic analysis, a glossary and onomastic indices. In order to better characterise the document's linguistic phenomenology comparisons are drawn with other Northern Italian Vernaculars (such as Veronese and Bresciano) and with the few known texts from medieval Trentino.
\end{abstract}

Keywords Northern Italian vernaculars. Trentino. Riva del Garda. Muta. Fifteenth century.

Sommario 1 Introduzione. - 2 II testo. - 3 Commento linguistico. - 3.1 Grafia. 3.2 Fonetica: vocalismo. - 3.3 Fonetica: consonantismo. - 3.4 Morfologia. - 3.5 Lessico. -3.6 Antroponimi e toponimi.

$\begin{array}{lll} & \text { Peer review } & \\ \text { Edizioni } & \text { Submitted } & 2021-08-03 \\ \text { Ca'Foscari } & \text { Accepted } & 2021-09-09 \\ & \text { Published } & 2022-02-17 \\ & \text { Open access } & \end{array}$

(C) 2022 | @( Creative Commons Attribution 4.0 International Public License 


\section{Introduzione}

L'Archivio di Stato di Trento conserva un interessante tariffario primoquattrocentesco relativo al dazio della muta di Riva del Garda. Alla muta rivana sono state dedicate diverse ricerche, perlopiù incentrate sul materiale ospitato presso l'Archivio comunale di Riva (cf. Sardagna 1884; Reich 1903; Grazioli 1987, 60-8; Martinelli 2000; si veda inoltre Malossini 2003, 36, 41, 59-60, 83, 101 nota 354, 160-3). Il documento di cui qui si tratta non è menzionato nei lavori succitati; al testo si è fatto invece cenno in due importanti saggi sulla storia economica e commerciale del Trentino (cf. Varanini [1996] 2020, 1088; [2004] 2020, 1129) e, con attenzione rivolta a fatti linguistici, in un intervento dedicato al Memoriale di Graziadeo di Castel Campo (cf. Magagna 1995, 292).

Proprio la lingua del documento è l'aspetto che interessa in questa sede: il testo ospita infatti una discreta quantità di brani e forme volgari rimasti finora inesplorati. Questa circostanza, sommata alla datazione del tariffario - tutto sommato alta, nel quadro complessivo dei documenti in antico trentino,$-{ }^{1}$ ci ha fornito ragioni sufficienti per impostare uno studio del manoscritto, di cui procureremo una descrizione, un'edizione parziale (§ 2) e per l'appunto una schedatura linguistica relativa alle parti in volgare (§ 3). Ragioni di spazio ci impediscono d'indagare problemi di tipo propriamente storico ed economico-fiscale (natura delle imposizioni, rapporto tra muta

Il saggio, pur scritto congiuntamente dai due autori, va così suddiviso a fini accademici: Stefano Cristelli §§ 1, 2, 3.2, 3.3, 3.5; Mario Wild §§ 3 (introduzione), 3.1, 3.4, 3.6. Siamo grati a Nello Bertoletti, Anita Malossini, Adriana Paolini e Luca Pesini, che ci hanno aiutato a risolvere alcune oscurità. Riferimenti archivistici: ASTn = Archivio di Stato di Trento; APV = Archivio del Principato Vescovile.

1 Per la questione ci permettiamo di rinviare a Cristelli (in preparazione), dove si proporrà l'edizione commentata di una nuova silloge di testi trentini antichi. Quanto a Riva del Garda, si ricorderà qui il significativo studio di Stenico (2013), che ha scoperto e pubblicato una carta in volgare del 1321 relativa proprio al territorio rivano; Malossini $(2003,176-81)$ ha edito un documento del 1385 - ma in copia primoquattrocentesca - in cui si leggono due suppliche rivolte ad Antonio della Scala rispettivamente dagli abitanti di Tenno e Riva. Nel corso della trattazione, anche per economia di spazio, il riferimento a questi e ad altri testi trentini sarà limitato ai fenomeni più notevoli. Si avverte che a due importanti documenti di area trentina, l'inventario giudicariese edito da Postinger (1901) e gli statuti dei Battuti di Trento pubblicati da Schneller (1881), si farà riferimento in forma abbreviata (Inventario giudicariese; Statuti dei Battuti) associando alle forme citate il numero di pagina della relativa edizione (si prescinde, per gli statuti, da Ressegotti 2010-11); anche lo zibaldone quattrocentesco studiato da Benedetti, Brugnolo (2002) sarà menzionato servendosi di un titolo accorciato (Zibaldone del Buonconsiglio). Un altro testo utile, il già ricordato Memoriale di Graziadeo di Castel Campo, sarà citato dall'originale (ASTn, APV, Sezione latina, capsa 68, nr. 226) facendo precedere all'indicazione di carta e rigo le lettere A o B, con riferimento rispettivamente alla prima metà del manoscritto (quella che reca, a c. $1 r$, la datazione più antica: 1400) o alla seconda (vergata capovolgendo il quaderno e ripartendo dal fondo). 
magna e muta parva, possibili identificazioni dell'estensore e di alcuni personaggi nominati nel manoscritto, ecc.), che lasceremo pertanto insondati. ${ }^{2}$ Questa prima ricognizione linguistica - particolarmente la sezione lessicale (§ 3.5) - potrà comunque agevolare chi, in futuro, vorrà dedicarsi a uno studio più approfondito del documento.

\section{Il testo}

ASTn, APV, Atti trentini, serie I, busta 76, fascicolo 14a; cartaceo, di 8 cc.; due filigrane, una del tipo 'testa di bue con occhi sormontata da fiore' (alle cc. 4 e 6, molto simile al nr. 14744 di Briquet 1923 [attestata a Würzburg nel 1405, ma forse di provenienza veneta]), l'altra del tipo 'testa di cervo con occhi sormontata da stella' (alle cc. 7 e 8, non identificata ma confrontabile, quanto al disegno, con i nrr. 82126-59 di Piccard 1987); misura mm $298 \times 209$ in media; cartulazione moderna a matita nell'angolo inferiore interno del recto; bianche le cc. $5 v-7 r$ e $8 r$; composto di un unico quaternione. Lo stato di conservazione è complessivamente buono, eccetto che per il foglio esterno: maggiormente degradata la c. $8 v$, per la cui lettura è talora indispensabile il ricorso alla lampada a ultravioletti; la c. 1 è inoltre priva dell'angolo inferiore esterno, ritagliato senza evidenti lacune testuali; tutte le cc. recano traccia di piegature nel senso della lunghezza (una, al centro, più marcata), ciò che spiega il maggior degrado di c. $8 v$, che dev'essersi trovata in posizione esterna. Il testo della tariffa della muta, datato marzo 1409 e di una sola mano, occupa le cc. $2 r$ - $5 r$; alle cc. $1 r$ e $8 v$, accanto ad alcuni brani in latino di varia natura (un solo scrivente primoquattrocentesco), una serie di note di credito, debito e vendita in volgare, coeve (una datata 21 dicembre 1409) e attribuibili paleograficamente all'autore della tariffa; ${ }^{3}$ la stessa mano è forse responsabile - oltre che di Muta in

2 Ci limiteremo a ricordare che il «feudo della Muda o Muta consisteva nel dazio che si esigeva a Riva su tutte le merci e generi che entravano ed uscivano dalla terra di Riva»; questo diritto, «risalente almeno al 1270» (se ne conserva l'atto di investitura da parte del vescovo di Trento), costituì a lungo «uno dei principali cespiti municipali» (Malossini 2003, 59 nota 179). Da notare che all'altezza del 1409 si era appena concluso un «momento decisivo per la storia tardomedievale di Riva», segnato dall'«assoggettamento ad altri poteri politici dell'Italia padana, quelli degli Scaligeri di Verona (1350-1387) e dei Visconti di Milano (dal 1387 al 1405; e in realtà [era stata] ancora la città dell'Adige - sede di un consiglio di governo per le "partes ultra Mincium" del dominio di Gian Galeazzo Visconti [...] - a costituire il punto di riferimento per il comune di Riva)» (Varanini [2011] 2020, 1161). Fino al 1440, anno della conquista da parte di Venezia (la cui dominazione terminò solo nel 1509), la cittadina fu soggetta, oltre che al principato vescovile trentino, alla contea del Tirolo e al ducato di Milano (cf. Malossini 2003, 78-9).

3 Ringraziamo la dottoressa Adriana Paolini, che ha confermato l'identificazione con una perizia sul testo di cui riproduciamo qui in nota il contenuto: «Le annotazioni del- 
Rypa alle cc. $1 r$ e $1 v$ e di Muta in Ripa a c. $8 v$, in scrittura calligrafica - di analoghe annotazioni in latino presenti a c. $7 v$, scritte in senso inverso rispetto al resto del documento e seguite da un frammento epistolare in tedesco di altro scriba quattrocentesco; da segnalare ancora, a c. $1 v$, un appunto archivistico d'epoca moderna (a matita, in tedesco) e il seguente testo, di difficile interpretazione (forse vergato dall'autore della lettera a c. 7v?): Car vegh pre sup(er) aug men | ypocrita mynor. Il documento è inedito.

Del manoscritto offriamo, come anticipato, una trascrizione parziale: riprodurremo anzitutto una breve porzione del tariffario (c. $2 r$ ), utile a dare un'idea della natura del testo e del rapporto che intercorre, nello stesso, tra latino e volgare (come si vedrà, quest'ultimo è impiegato sempre e solo per indicare le merci e, talora, la quantità sottoposta a tassazione); pubblicheremo poi integralmente le annotazioni in volgare presenti alle cc. $1 r$ e $8 v$ (trascurando le altre scritture ivi presenti). ${ }^{4}$

le cc. $1 r$ e $8 v$ sono della stessa mano che trascrive con una scrittura più posata il tariffario. La morfologia delle lettere varia anche all'interno del testo principale e si possono ritrovare forti analogie con le lettere delle note: dalla $F$ maiuscola al modo di segnare Item, al compendio per pro, alla $z$ a forma di 3 ecc.» (comunicazione personale dell'11 maggio 2021).

4 I criteri, validi anche per i brani qui non editi ma citati nel $\S 3$, sono i seguenti: divisione delle parole, punteggiatura, diacritici, maiuscole e minuscole corrispondono all'uso moderno; indichiamo il cambio di rigo con una barra verticale, doppia in corrispondenza dei righi 5, 10, 15, ecc. (segnalati, insieme al primo, anche tramite numerazione con cifra in esponente); tra parentesi tonde si trovano le lettere frutto di scioglimento di segni abbreviativi (nel caso di $c o(m)$ prà si opta per $m$ in considerazione dell'assenza di forme con $m$ o $n$ a piene lettere davanti a labiale); per dar conto di un'integrazione si ricorre alle parentesi quadre, per segnalare i depennamenti dello scrivente alle parentesi angolari invertite; inseriamo tre punti spaziati in corrispondenza delle porzioni di testo che, nonostante il ricorso alla lampada di Wood, risultano illeggibili; $v$ è distinta da $u ;<j>$ è resa con $i$ tranne che nei casi in cui indica l'unità o l'ultima unità nelle cifre; le preposizioni articolate sono stampate univerbate; distinguiamo graficamente $a$ 'a' da à 'ha', de 'di' da de' 'deve', dè 'diede'; i numeri romani sono riprodotti in maiuscoletto; la nota tironiana a forma di 7 è sciolta in contesto volgare con $e$ (non si ha mai et a piene lettere); il tratto orizzontale con cui lo scrivente collega alcune zone del testo è reso, a prescindere dalla lunghezza effettiva, con un trattino lungo; manteniamo inalterate le abbreviazioni dei nomi dei mesi, il simbolo $\div$ per 'mezzo' e le seguenti altre forme: car. 'carati', dat. 'date, -i', d. e dr. 'denaro, -i', duc. 'ducati', fass. 'fascio', gal. 'gallete' (misura di volume), gros. 'grosso' (moneta), libr. 'libbra, -e', m. 'maestro', mod. 'moggio, -i', r. segno di avvenuto pagamento, res. 'resto', s. 'soldo, -i', t. e trid. 'trentino, -i', v(er). e v(er)on. 'veronese, -i'; si scioglie con (once) il segno a forma di chiocciola presente a c. $8 v 22$, simile a quelli registrati - per l'appunto con il valore di 'oncia, -e' - in Cappelli 2011, 410, colonna di destra; non sembrano esserci alternative utili allo scioglimento proposto per $p$ (rim)o $8 \mathrm{v} 13$, anche se il $\mathrm{ms}$. reca, in luogo dell'atteso $p^{\circ}$, un insolito po; nel caso del toponimo $\mathrm{Gar}^{\circ}$, l'assenza di proposte di scioglimento sicure impone di mantenere la forma abbreviata. Nel riportare i brani del tariffario distinguiamo tra volgare e latino stampando il primo in tondo e il secondo in corsivo (le indicazioni di prezzo, sempre in forma abbreviata, sono attribuite al latino); nel caso delle annotazioni, dove la distinzione è talora difficile o impossibile, si rinuncia ad adottare la stessa soluzione. Alcune delle scritture a c. $8 v$ sono state vergate nel margine destro in relazione a prece- 
Stefano Cristelli, Mario Wild

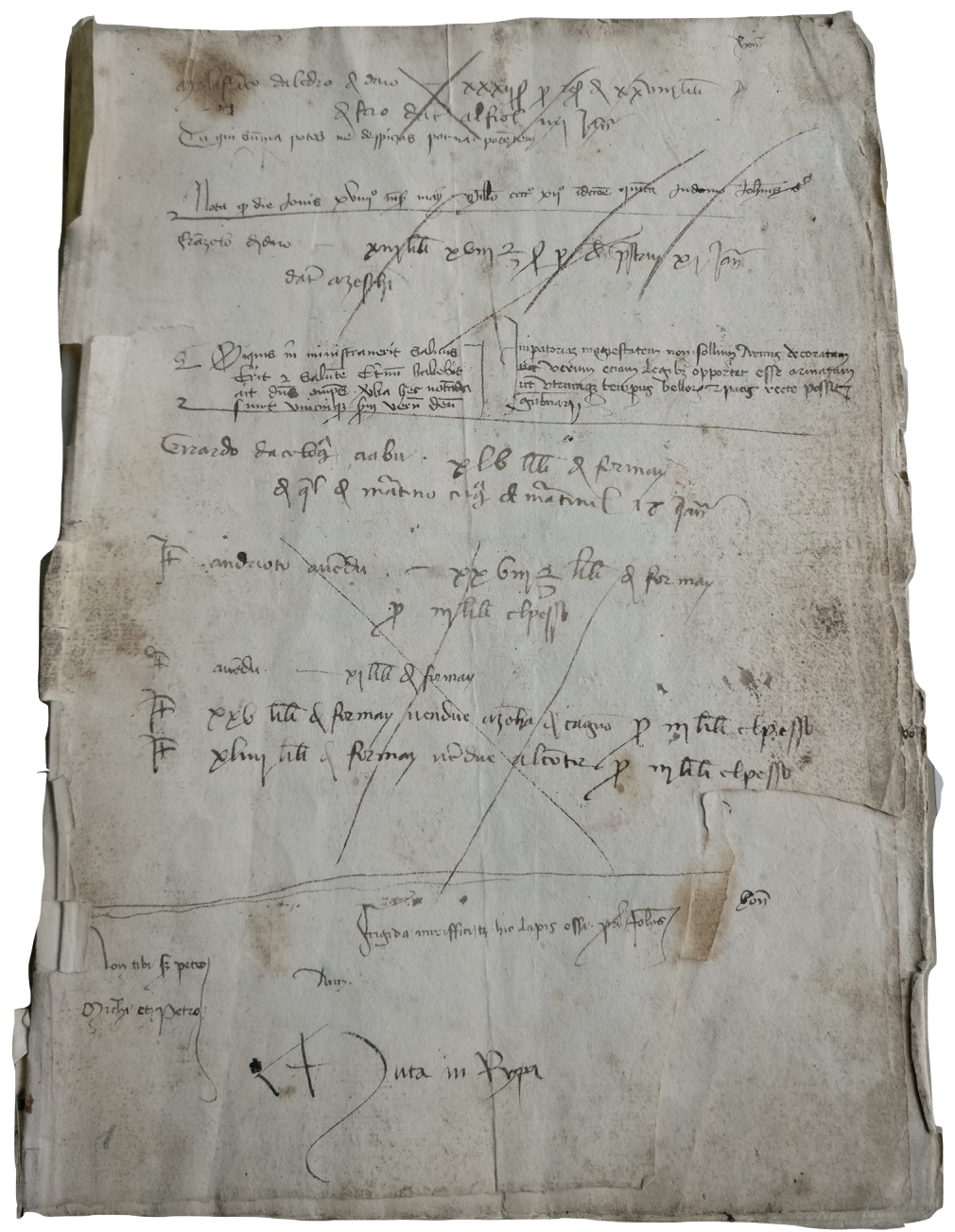

Figura 1 ASTn, APV, Atti trentini, serie I, busta 76, fasc. 14a, c. $1 r$ 
Stefano Cristelli, Mario Wild Il volgare trentino in una tariffa rivana del 1409

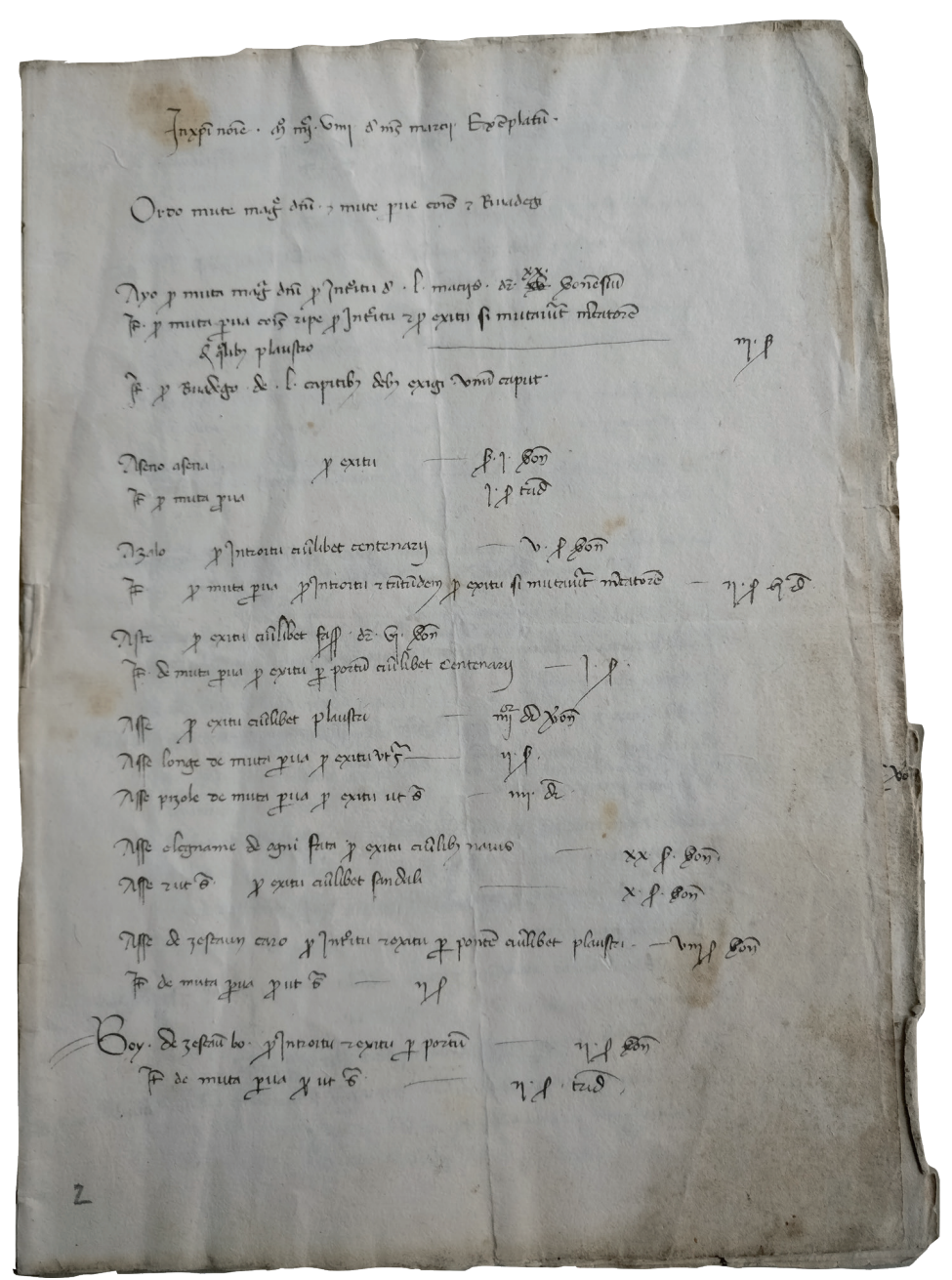

Figura 2 ASTn, APV, Attitrentini, serie I, busta 76, fasc. 14a, c. $2 r$ 


\section{Testo della tariffa (parziale: c. $2 r$ )}

${ }^{1}$ In (Christ)i no(m)i(n)e. M IIIJ $^{c} \operatorname{VIIIJ} d(e)$ m(en)s(e) marcii exe(m)platu(m). |

Ordo mute mag(n)e d(omi)ni (et) mute p(ar)ve co(mun)is (et) rivadegi. |

Ayo $p$ (ro) muta mag(n)a d(omi)ni $p$ (ro) int(r)oitu d(e) L maciis $d r$. $>\mathrm{XV}<$ $\mathrm{xx} v(\mathrm{er}) \mathrm{one}(\mathrm{n}) \operatorname{siu}(\mathrm{m})$. |

It(em) p(ro) muta p(ar)va co(mun)is Ripe p(ro) int(r)oitu (et) p(ro) exitu si mutav(er)it m(er)catore $(m) \|{ }^{5} d(e) q(u) o l i b(e t)$ plaustro - IIJ s. | It(em) p(ro) rivadego de L capitib(us) deb(et) exigi unu(m) caput. | Aseno, asena $p(r o)$ exitu - s. J v(er)on. |

It(em) $p$ (ro) muta p(ar)va J s. trid.

Azalo p(ro) introitu cui(us)libet centenarii - vs. v(er)on. \|

${ }^{10}$ It(em) $p$ (ro) muta $p$ (ar)va $p$ (ro) introitu (et) ta(n)tu(n)dem p(ro) exitu si mutav(er)it m(er)catore $(m)$ - IJ s. vJ d. |

Aste $p(r o)$ exitu cui(us)libet fass. dr. vJ v(er)on. |

It(em) de muta $p$ (ar)va $p$ (ro) exitu $p(e r)$ portu(m) cui(us)libet centenarii - J $s$.

Asse $p($ ro) exitu cui(us)libet plaustri - IIIJor dr. v(er)on. |

Asse longe de muta p(ar)va p(ro) exitu ut s(upra) - IJ s. \|

${ }^{15}$ Asse pizole de muta $p$ (ar)va $p$ (ro) exitu ut s(upra) - IIJ dr. I

Asse e legname de ogni fata $p$ (ro) exitu cui(us)lib(et) navis - Xx s. v(er)on. | Asse (et) ut s(upra) p(ro) exitu cui(us)libet sandali - X s. v(er)on. |

Asse de zescaun caro $p$ (ro) int(r)oitu (et) exitu p(er) ponte(m) cui(us)libet plaustri - VIIJ s. v(er)on. |

It(em) de muta p(ar)va p(ro) ut s(upra) - IJ s. \|

${ }^{20}$ Boy de zescau(n) bo' $p$ (ro) introitu (et) exitu p(er) portu(m) - IJ s. v(er)on. $\mid$

It(em) de muta p(ar)va p(ro) ut s(upra) - IJ s. trid.

3. $\mathrm{XX}$ ] aggiunto sopra la cifra depennata. 


\section{Annotazioni in volgare a c. $1 r$}

${ }^{1}$ M. Lafra(n)co da Ledro d(e') daro - xxxij s. p(ro) res. d(e) XxVIIJ libr. | d(e) fero dat. al fiol III ian. |

Fra(n)zolo d(e') daro - XIIJ libr. XVIIJ $\div$ s. p(er) d. p(re)stay XJ ian. | dat. a Zeschi(n). ॥

${ }^{5}$ Girardo da Colog(n)a à abù XLV libr. d(e) formay | d(e) q(ue)l d(e) Ma(r)tino $\operatorname{cug}(n)$ à de Ma(r)tinel 18 ian. |

It(em) Andrioto à ve(n)dù - XXVIJ $\div$ libr. d(e) formay | p(ro) IIJ libr. el pesso. |

It(em) à ve(n)dù - XJ libr. d(e) formay. ॥

${ }^{10}$ It(em) xxv libr. d(e) formay vendue a Zoha(n) d(e) Cagno(n) p(ro) IIJ libr. el pesso. |

It(em) XLIIIJ libr. d(e) formay ve(n)due al Co(n)ter p(ro) IIJ libr. el pesso.

6. ian.] i sembra il risultato di una correzione.

\section{Annotazioni in volgare a c. $8 v$}

${ }^{1}>$ No < Nicolò d(e) Enselmo de' av(er) - XXX libr. VIIJ s. p(ro) | XVJ gal. d(e) sal p(er) xxxij s. gal. | R. vj libr. | It(em) xJ s. dat. || ${ }^{5}$ a Andrea. | It(em) IIIJ s. dat. ai | mesuradori. |

Ava(n)cino e Iache(m) d(e') daro p(er) XJ mod. (e) viJ gal. | d'olio p(ro) xxv libr. mod. xxJ dece(m)br. 1409. ॥

${ }^{10}$ R. XxxvJ duc. |

Resta XVIIJ duc. ... J gros. |

Ava(n)cino e Iachem d(e)la Val da No(n) d(e') daro: | p(rim)o - LXVIIIJ duc. IIJ libr. p(er) xv mod. ol(e)i | p(er) xxv libr. . . . s. mod. ano xxx dece(m)br. || ${ }^{15}$ R. IJ duc. | da Ava(n)cin | dì s(upra)s(crip)to. | R. LxxJ libr. | p(er) coramo vendù $\mid{ }^{20}$ a Ventura da Gar ${ }^{\circ}$. R. xviIj car. p(ro) xviIJ | libr. VJ (once) d(e) sonz(a) de | ulti(m)o dece(m)br. |

R. XIJ duc. i q(u)ai à dà Iache(m) || ${ }^{25}$ del Ligo XIIJ ian. |

It(em) $>$ de' $^{\prime}$ av(er) < de' dar Zoha(n) fradel de Ava(n)cin xxxJ | libr. p(er) resto $d(e) v$ mod. ol(e)i ano XIIJ ian. |

Ventura da $\mathrm{Gar}^{\circ}$ de' daro - Xxvij libr. p(ro) resto d(e) coramo | co(m)prà da Ava(n)cin e seg(n)à ala soa raxo(n). ॥

${ }^{30}$ It(em) IIIJ libr. v s. p(ro) IJ pelle che ge dè Peder d(e) Nicolò da Brezo. | It(em) IIJ libr. XV s. p(ro) . . . ch(e) ge dè el fradel d(e) Ava(n)cin. | R. J duc. d'or IIIj ian. | R. IJ duc. vj ian. | R. xviuj libr. x s.

1. Enselmo] la seconda e forse corretta su altro.

6. It(em)] il primo tratto della tè appoggiato al corpo della lettera precedente, ma risulta comunque distinguibile.

11. Resta XVIIJ duc.... J gros.] più a destra, in prossimità del margine, un appunto frammentario di lettura non del tutto chiara a causa di un guasto del supporto: XLVIIJ, 
con J di lettura incerta, e al disotto la sal XIIJ duc. seguito da alcune lettere depennate (forse LIJ?).

26. > de' av(er)<] segue, coperta dallo stesso depennamento, una o o un'altra lettera lasciata incompiuta (d?). La distanza da av(er) è comunque sufficiente per escludere la lettura av(er)o.

30. Nicolò] lettura incerta: forse Nicalò.

\section{Commento linguistico}

Presentato il documento, veniamo all'analisi linguistica, che articoleremo distinguendo fatti grafici (§ 3.1), fonetici (§§ 3.2 e 3.3) e morfologici (§ 3.4) per poi registrare lessico (§ 3.5) e forme onomastiche (§ 3.6); vista la natura della fonte, non si proporranno osservazioni di sintassi.

Come si vedrà, la fisionomia del testo è sufficientemente caratterizzata da escludere l'appartenenza dello scrivente non solo all'area lombardo-orientale, ma anche a quella veronese, considerate microspie quali la mancata sincope nei proparossitoni lareso, pegora e pevero o la presenza - pur circoscritta a nomi propri - di forme anaptittiche come Co(n)ter e Peder, che presuppongono l'apocope della vocale finale dopo un nesso di occlusiva e vibrante; si considerino anche le diverse attestazioni di formay, con riduzione -io $>-i$ (documentabile solo con difficoltà a Verona: cf. Bertoletti 2005, 161). Il testo, insomma, può essere attribuito al Trentino anche su base linguistica, non solo quanto al contenuto e alla menzione di toponimi locali nelle annotazioni alle cc. $1 r$ e $8 v$; allo stato attuale delle conoscenze sui volgari trentini, rappresentati da un numero ridotto di documenti legati a poche aree regionali, sembra comunque sconsigliabile tentare una localizzazione più precisa.

Si avverte che le occorrenze saranno citate sempre con riferimento alla c. e al r.; se una stessa forma ricorre più di quattro volte, ci si limita a indicare i luoghi delle prime tre attestazioni facendo seguire il numero totale (indicato con $t$.) tra parentesi tonde. Nei §§ 3.1-3.4 ci si avvarrà solo eccezionalmente di glosse e sarà rara l'indicazione delle basi etimologiche; per chiarire l'interpretazione delle singole voci si può fare riferimento al glossario (§ 3.5). Si noti ancora che in alcuni casi (ad es. mel de q(u)olib(et) modio p(ro) introitu d(e) alieno ep(iscop)atu - xx dr. v(er)on. $3 v 8$ ) il nome della merce tassata, data l'ambiguità tra latino e volgare, potrebbe risultare inutilizzabile ai fini del commento linguistico (ci si può chiedere, in altre parole, se non si tratti semplicemente di una forma latina); che si abbia di fronte un'attestazione volgare pare comunque assicurato dal fatto che, là dove non sussistono dubbi simili, le porzioni di testo che ospitano l'indicazione dei beni sottoposti al dazio non si presentano in nessun caso in latino. 


\subsection{Grafia}

L'occlusiva velare sorda è resa sempre con $<\mathrm{c}>$ davanti ad a (Cagno(n) 1r10, capeli 'cappelli' 2v27, carne 2v24, 2v26, case 'casse' 3v1, castag(n)e 3r4, m(er)candaria 5r21, ecc.), mentre davanti a vocale

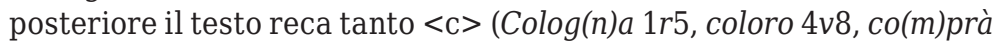
8v29, Co(n)ter 1r11, coramo 8v19, 8v28, cori 2v12, 2v15, cug(n)à 1r6, fianco 3r13, Lafra(n)co 1r1, Nicolò 8v1, 8v30, porco 3v6, saco 3v21, $4 r 26,4 v 1$, scudi $5 r 5$ ) quanto, pur in un solo caso, <ch > (becho 2v8); prima di vocale palatale si trova $<\mathrm{ch}>$ (che $8 v 30$, Iache $(m) 8 v 8,8 v 24$, -m $8 v 12$, vache $2 v 24,4 v 13$, Zeschi(n) 1r4). La sonora ricorre in tutti i contesti come $<\mathrm{g}>$ : Garzaria 2v15, i(n)tregi 2v20, -o 3v11, largà $3 r 24$, legumi 3v5, longe 2r14, orbage 3v26, 4r3, 4r5, pegola 4r28, pegora $4 r 7,4 r 20 .^{5}$

Se non si tratta di un suono intermedio ([c]) tra la conservazione e la completa risoluzione del nesso con laterale, troviamo <chi> a rappresentare l'affricata palatale sorda in schiete $5 r 11$, sechie $5 r 9,5 r 11$.

Per quanto riguarda la resa dell'affricata dentale (sorda e sonora), il testo ricorre sempre a $<\mathrm{z}>$, mai a $\langle$ ç> : bronzo 2v1, falze 3r21, 4r24, Fra(n)zolo 1r3, ma(n)zo 2v12, 2v24, mezani 2v20, Zeschi(n) 1r4, ecc. Andrà letta con affricata dentale sorda una serie di esempi in cui, in conformità con la grafia del (medio)latino, troviamo $<\mathrm{c}>$ : Ava(n)cin 8v16, 8v26, 8v29, 8v31, Ava(n)cino 8v8, 8v12, cera 2v10, Cipri 4v21, civole $3 r 1$, porci $4 r 6,{ }^{6}$ specificada $5 r 21$. Risponde probabilmente a un uso conservativo anche la $\langle\mathrm{g}>$ impiegata, per la sonora, in $\mathrm{Gi}$ rardo 1 r5.

La nasale palatale è resa con <gn> in agnelo 4r20, Cagno(n) $1 r 10$, legname 2r16, -o 3v1, legno 5r11, ogni 2r16, 4r20, scalogne 5r17; si hanno invece $g^{a}, g^{e}, g^{o}, g^{i}$ nei seguenti casi: castag(n)e 3r4, Colog(n)a 1r5, cug(n)à 1r6, og(n)i 2v3, 3v1, 3v21 (t. 5), pig(n)olà 4r12, 4r15, pug(n)o 3v21, 4v1, seg(n)à 8v29.

Alla sibilante sorda intervocalica corrisponde <ss > in asse $2 r 13$, $2 r 14,2 r 15$ (t. 6), pesso 'pesce' 4r13; altrove si registra <s>: case 'casse' $3 v 1$, grosa $4 v 21$, -i $2 v 18$. Si rileva l'impiego del grafema $<x>$ per [s] in brexa(n) 3v24, 3v27 ed exir 4v13, forme giustificate dal modello latino; $<\mathrm{x}>$ anche nel dotto Alexandria $4 v 21$, d'interpretazione fonetica meno immediata. La sibilante sonora è resa con $<$ s $>$ nella maggior parte dei casi: asena $2 r 7$, -o $2 r 7$, lareso $3 r 6,3 r 10$, mesurado-

5 Da segnalare a parte il pronome ge $8 v 30,8 v 31$, la cui interpretazione fonetica non è univoca (vedi nota 31 ).

6 È vero che nel Memoriale di Graziadeo di Castel Campo, vergato nei primissimi anni del Quattrocento nell'altipiano del Lomaso (una ventina di chilometri a nord di Riva), alle cc. A3r e $v$ si legge diverse volte po(r)chi; pare tuttavia oneroso ammettere, per l'esempio del nostro testo, una lettura [k] del grafema $<\mathrm{c}>$ davanti a vocale palatale (come si è visto, del resto, davanti a $e$ e $i$ la velare è resa soltanto con $<\mathrm{ch}>$ ). 
ri $8 v 7$, presoro $4 r 22$, rasa $4 v 2$, roso $3 r 15,{ }^{7} v($ er)oneso $3 v 22,3 v 27$; con $<\mathrm{x}>$ in noxe $3 v 21$, raxo(n) $8 v 29$ e pexo 'peso' $3 r 24$, cui si affianca però, più spesso, la variante pesso $1 r 8,1 r 10,1 r 11$ (t. 5).

Si nota la presenza di $<y>$ in ayo $2 r 3$ e in boy $2 r 20,2 v 24$, formay $1 r 5,1 r 7,1 r 9$ (t. 5), $p(r e) s t a y 1 r 3$ (però ai $3 v 1,8 v 6$, foia $3 r 15$, formaio $3 r 11$, ecc.).

\subsection{Fonetica: vocalismo}

Trascurando le sorti di I e Ū, che non presentano particolarità (si trovano rispettivamente $i$ e $u$ ), mette conto osservare l'assenza di dittongamenti spontanei a partire da $\breve{\mathrm{E}}$ e ŏ (si considerino dè 'diede' $8 v 30,8 v 31$, Peder $8 v 30$, pegora $4 r 7,4 r 20$, prede 'pietre' $4 r 24$; fiol 1r2, Fra(n)zolo 1r3, mola 3v15, 3v17, 3v19, nove 'nuove' 4v15); le stesse vocali non presentano alterazioni dovute a condizionamenti metafonetici. Analoghi gli sviluppi di $\overline{\mathrm{E}}, \breve{\mathrm{I}}$ e ō, Ŭ, che - tolto il cultismo capitoli $5 r 21$ - dànno rispettivamente $e$ e $o$ (anche in presenza di -i: canteri $4 v 4$, peri $4 r 26$; mesuradori $8 v 7$, pomi $4 r 27$ ); nessuna alterazione, a partire da *-ōRJU(M), in presoro $4 r 22 .{ }^{8}$ Da notare che [o] resta intatta anche davanti a nasale: cf. ad es., da -ōNE(M), Cagno(n) 1r10, castro(n) $2 v 17,4 r 20$, monto(n) $4 r 20$, raxo(n) $8 v 29 .{ }^{9}$

Quanto ad A tonica, la vocale è sempre conservata; notevole l'esito non palatalizzato di -ARJU(M) in zentenaro $2 v 1$, che avvicina il testo alle condizioni del veronese più che a quelle del lombardo orientale, dove lo sviluppo normale è a -er (cf. Bonelli, Contini 1935, 142; Ghinassi [1965] 2006, 91; Bertoletti 2005, 162-4; Tagliani, Bino 2011, 114). ${ }^{10}$

Da AU tonico Nicolò $8 v 1,8 v 30$, or $8 v 32$, soma 3r21, 4r11, 5r21.

Per le vocali toniche in iato notiamo l'assenza di alterazioni in $A n$ drea $8 v 5$, bo' $2 r 20$ (con apocope di -e), boy $2 r 20,2 v 24$ e soa $8 v 29$;

7 Per la pronuncia di questa forma ci si avvale del confronto con forme moderne quali ['rozolo], ['rozola] 'scotano, sommacco' in Rigobello, s.v. ròfola.

8 I dati sono in linea con quelli del corpus di testi trentini analizzati in Cristelli (in preparazione), che si mostrano immuni da fenomeni di dittongamento e che solo marginalmente sono toccati dalla metafonia. Si ricorderà che i volgari di Brescia e Verona sono accomunati dall'assenza di dittongamento, mentre differiscono quanto all'incidenza delle chiusure metafonetiche (solo -et $(h)<-$ ATİ a Brescia di contro a una più massiccia presenza del fenomeno a Verona, dove pure, come a Vicenza, si ha -oro <-ōRJU(M)); per quest'ultimo aspetto in particolare cf. Bertoletti 2001, 238-9; 2005, 42-50.

9 Per la chiusura di [o] tonica davanti a nasale nel vicino volgare veronese, e più in generale nell'Italia del Nord, cf. Bertoletti 2005, 51-2; 2007, 43 nota 52.

10 L'opposizione si conserva modernamente (cf. Bonfadini 1983, 32). In questo quadro il Trentino rappresenta un'area di transizione: la palatalizzazione di -ARJU(M) «si estende a tutto il Trentino occidentale, compreso il Medio e Basso Sarca (dove abbiamo però qualche infiltrazione di -àr)»; interessa anche il Roveretano, «ma è estranea al nucleo centrale che testimonia compattamente -àr» (cf. Bonfadini 1992, 39). Tale situazione è in parte rispecchiata dai testi commentati in Cristelli (in preparazione). 
cf. invece $i<e$ in osia $3 v 15 .{ }^{11}$ Quanto ai gruppi vocalici secondari -ao, -uo, ecc., si registra:12 da -ATU(M) Co(m)prà 8v29, cug(n)à 1r6, largà 3r24, lavorà 3r13, $4 r 17$ bis (t. 5), pig(n)olà 4r12, 4r15, scartà 3r19, seg(n)à 8v29; da -ATI p(re)stay 1r3; da -ATAE lavorè 3r5; da -UTU(M) vendù $8 v 19$; da -UTAE vendue $1 \mathrm{r} 10,-(n)-1 r 11$.

In protonia si trova $i<\bar{I}$ in Nicolò $8 v 1,8 v 30$ e pig(n)olà $4 r 12,4 r 15$; nessun esempio per $\overline{\mathrm{U}}$. Quanto a $\breve{\mathrm{I}}$ e a $\overline{\mathrm{E}}$, $\breve{\mathrm{E}}$, si ha di norma $e$ : brexa(n) $3 v 24,3 v 27$, legname $2 r 16$, -o 3v1, legumi 3v5, mezene 3v6, pelame $4 r 17,4 r 21$, vedelo $2 v 26,4 r 20$, ecc.; si trova $i$ in civole $3 r 1$, dove il timbro è giustificato dalla vicinanza di c- (cf. Ghinassi [1965] 2006, 65; ma si veda la nota 11), nel dotto specificada 5 r21 e in distin[a]to, altro esempio di trattamento culto quanto alla $i$ immediatamente protonica, ma interessante soprattutto per la presenza di dis- < DES-; da segnalare, per quanto riguarda le serie prefissali, anche $i(n)$ - in $i(n) d i$ 'nei' $5 r 21$ e in i(n)tregi $2 v 20,-o$ 3v11. In protonia sintattica si trova sempre la preposizione de, attestata passim molte volte. Da Ŭ e ō, ŏ solo o (coloro 4v8, lavorà 3r13, $4 r 17$ bis [t. 5], -è 3r5, molino 3v17, ecc.; davanti a nasale: co(m)prà $8 v 29$, monto(n) $4 r 20)^{13}$ eccetto che in $\operatorname{cug}(n) a ̀$ 1r6, forma ben nota in area settentrionale, nella quale l'innalzamento è dovuto all'elemento palatale attiguo (cf. ad es. Bertoletti $2005,88) ; i^{14}$ come si è già osservato in apparato nel $\S 2$, non è sicuro

11 Si avverte qui che nel caso di civole $3 r 1$, in mancanza di altri elementi, si sceglie di leggere la voce come un parossitono, per quanto nei dialetti moderni di area trentina le forme di 'cipolla' possano essere generalmente riportate a CEPŬLA (cf. LEI, 13, 973-4). Se anche per la nostra forma valesse quest'ultimo etimo, bisognerebbe postulare il dileguo di - $v$ - < - P-, la chiusura in iato secondario della tonica - con la conseguenza concreta di dover schedare la forma in questo capoverso - e la successiva ricomparsa di - $v$ - come consonante epentetica antiiatica.

12 E noto che il trattamento dei gruppi vocalici finali secondari ha carattere individuante nel quadro degli antichi testi veneti: cf. al proposito Tomasin 2004, 111-13; Bertoletti $2005,64,76$. In area trentina il problema si lega a un altro aspetto particolarmente delicato, vale a dire agli esiti delle dentali intervocaliche, ancor oggi più o meno conservativi a seconda della vallata (cf. ad es. Mastrelli Anzilotti 1992, 8-9 e 17; l'Alto Garda rientra nella zona in cui - $d->$ Ø: cf. Battisti, Ventura 1955, 13). Da questo punto di vista, le attestazioni antiche devono essere valutate caso per caso: per es., quando l'Inventario giudicariese offre fruà, spinà, spadolà, ecc., non di riduzione da -ao si tratterà, visto che altrove si hanno solo - $a d a$, -ade, -adi, ma di apocope di -o in -ado e successivo ammutolimento della dentale riuscita finale; diverse le conclusioni a cui si giunge analizzando gli Statuti dei Battuti, che allato ad -à < -ATU(M) e -ù < -UTU(M) recano -ay

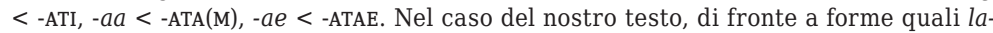
vorè (con contrazione nota anche all'area veronese: cf. Bertoletti 2005, 71), p(re)stay e vendue, -(n)- non si può che riportare -à ad -ao e -ù a -uo. Nella trattazione non si considerano le forme abù $1 r 5$, dà $8 v 24$, ve(n)dù $1 r 7,1 r 9$ per l'impossibilità di stabilirne con certezza il genere e il numero; includiamo lavorà 4r21 bis tra gli ess. di -ATU(M), ma, visto il contesto, non si può escludere a rigore che si tratti di -à < -ATA(M): pelame d(e) monto(n), cavra, cavre, pegora, castro(n), agnelo, vedelo e d(e) ogni pele menuda, lavorà e no lavorà, d(e) zescauna pelle - J dr. $4 r 21$.

13 Per la possibile chiusura di [o] protonica davanti a nasale cf. Bertoletti 2005, 87.

14 Si veda anche cugnà B5r23, B9r4 nel Memoriale di Graziadeo di Castel Campo. 
Nicolò $8 v 30$, che potrebbe essere trascritto Nicalò (con una dissimilazione che, per questo antroponimo, non sarebbe priva di riscontri: vedi § 3.6). È sempre conservata A protonica (da considerare a parte zescaun $2 r 18,2 v 1,-(n) 2 r 20,2 v 18,2 v 20$ [t. 14] e zescauna $3 r 21$, $4 r 11,4 r 15$ [t. 9], gallicismi: cf. Rohlfs, § 501); si noti a questo proposito anche il mantenimento di ar intertonico in m(er)candaria $5 r 21$. Da AU protonico si giunge a o in lorino 3v27, orbage 3v26, 4r3, $4 r 5$.

Per le vocali atone in iato si segnalano gli antroponimi Andrioto $1 r 7$, con innalzamento di e protonica, e il conservativo Zoha(n) $1 r 10,8 v 26$.

Quanto alle vocali postoniche, si ha $e$ da I ed Ӗ: asena $2 r 7$, -o $2 r 7$, lareso $3 r 6,3 r 10$, pevero $4 r 9 .{ }^{15} \mathrm{Da}$ Ŭ e ŏ si ha $o$ in capitoli $5 r 21$, pegola $4 r 28$, pegora 4r7, 4r20; notevole Iache $(m) 8 v 8,8 v 24,-m$ 8v12 (su cui vedi § 3.6). Come si ricava dagli esempi citati, il testo non reca forme sincopate del tipo di quelle attestate in veronese antico (larzo, pegra, pevro: cf. Bertoletti 2005, 109-13); è comunque degna di nota la caduta della postonica in masna $3 v 15 .{ }^{16}$

Venendo al trattamento delle vocali atone finali, che rappresenta un aspetto particolarmente delicato, ${ }^{17}$ osserviamo anzitutto che tanto - $a$ quanto -e e -i morfi plurali risultano sistematicamente mantenuti (i si conserva anche in ogni, og(n)i, per le cui occorrenze vedi § 3.4); isolato il caso del maschile plurale ingeste $2 v 22$ (germanismo: vedi $\S 3.5$, s.v. ingesto), da spiegare forse come un errore dello scrivente.

La vocale $e$, quando non è marca di plurale, può andare incontro a 1) conservazione (dopo l < L e LL: barile 4r28, pele 4r20, pelle 4r21; dopo lz: falze 4r24; dopo m: legname $2 r 16$ [allato a legnamo $3 v 1$, per cui cf. infra], pelame 4r17, 4r21; dopo rn: carne 2v24, 2v26; dopo v: nave $3 v 1$ ); 2) apocope, attestata con sicurezza solo dopo laterale (mel $3 v 8$, sal $4 v 18,4 v 21,8 v 2$ [cf. anche sal registrato in apparato nel § 2], Val $8 v 12$ ) e vibrante (dar $8 v 26$ ed exir $4 v 13),{ }^{18}$ mentre, prescindendo dall'arabismo oton $4 r 1$, non si può fare affidamento su forme compendiate come Cagno(n) 1r10, castro(n) 2v17, 4r20, monto(n) 4r20, raxo(n) $8 v 29$, Zoha(n) $1 r 10,8 v 26$; 3) sostituzione con $o$, fenomeno esemplifi-

15 Si aggiungano, in contesto latino, rivadegi $2 r 2,-o$ 2r6, 3r3, $3 r 4$ (t. 9).

16 Per il resto la sincope è attestata soltanto in protonia: co $(m)$ prà $8 v 29$, largà $3 r 24$. Quanto alle forme di 'larice' e 'pecora', vale la pena di segnalare la presenza di occorrenze non sincopate anche nell'Inventario giudicariese (ad es. larexo 27, pegora 28).

17 In quanto il Trentino doveva rappresentare un'area di transizione fra la Lombardia orientale, in cui l'apocope aveva raggiunto uno stadio molto avanzato, e il territorio veneto (veronese in specie), maggiormente conservativo; alle particolari condizioni del volgare di Verona, caratterizzato dalla presenza di un'-o (anche per - $e$ ) non restitutiva, ma fonologicamente presente, sono state dedicate importanti osservazioni da parte di Bertoletti $(2005,116-37)$. Sulla complessa storia delle atone finali in Italia settentrionale cf. Loporcaro 2005-06.

18 Inutile la forma compendiata $a v(e r) 8 v 1,8 v 26$. 
cato non solo da una serie di maschili di III declinazione, poco utili all'analisi in quanto potenzialmente riconducibili a metaplasmi (azalo $2 r 9$, coloro $4 v 8$, coramo $8 v 19$, 8v28, lareso 3r6, 3r10, legnamo 3v1, pesso $4 r 13$, pevero $4 r 9$, ramo 4 r30), ma anche dagli infiniti amolaro $3 v 19$, molaro $3 v 15$ e daro $1 r 1,1 r 3,8 v 8$ (t. 5). ${ }^{19}$ Per alcune delle forme apocopate è evidente il concorso del contesto prosodico (cf. in particolare sal menuda 4v18, sal grosa $4 v 21$ e Val da No(n) 8v12).

Quanto a -o, le forme che la presentano intatta sono numerose; la vocale ricorre tanto dopo nesso consonantico (per es. bronzo $2 v 1$ e ma(n)zo 2v12, 2v24, fianco 3r13, lardo 3r23, Ledro 1r1, porco 3v6) quanto dopo consonante singola (non solo in casi come Andrioto 1r7, i(n)trego 3v11, mozo 3v22, 3v24, ecc., ma anche, dopo sonante, in mulo $3 v 11,3 v 13$, vedelo $2 v 26,4 r 20$, Ava(n)cino 8v8, $8 v 12$ [accanto ad Ava(n)cin citato oltre], molino 3v17, vino 4v10, fero $1 r 2,2 v 27,3 r 17$ [t. 5], zentenaro $2 v 1$, ecc.); visto quel che si dirà infra circa la riduzione -io > -i, cf. anche ayo 2r3, formaio 3r11 e olio 3r10, 3v22, 3v24 (t. 6). La caduta di - o è attestata dopo $l$ (< L e LL; oltre a el 1r8, 1r10, 1r11, 8v31, al 1r2, 1r11, del 8v25, cf. fiol 1r2, fradel 8v26, 8v31, Ma(r)tinel 1r6, q(ue)l 1r6), m (Iachem 8v12), ${ }^{20} n$ (Ava(n) cin 8v16, 8v26, 8v29, $8 v 31$, zescaun $2 r 18,2 v 1){ }^{21} r$ (or $8 v 32$ ) e, con sviluppo più tipicamente lombardo, dopo iod (formay 1r5, 1r7, $1 r 9$ [t. 5]) e nesso di dentale e vibrante $(\mathrm{Co}(n)$ ter $1 \mathrm{r} 11$, Peder $8 v 30$, con epentesi di una vocale d'appoggio: cf. il paragrafo seguente). Un certo numero di forme può trovare spiegazione nel contesto fonosintattico (zescaun, ad es., ricorre solo come aggettivo preposto al sostantivo: de zescaun caro 2 r18, de zescaun zentenaro $2 \mathrm{v} 1$ ); è nondimeno possibile individuare alcuni esempi in posizione sicuramente prepausale (è il caso di Ava(n)cin $8 v 31$ e formay $1 r 9$ ).

In generale, è da osservare la differenza tra la lingua della tariffa e quella delle annotazioni alle cc. $1 r$ e $8 v$ : sono infatti prevalentemente queste ultime a presentare forme apocopate (nella tariffa unicamente exir $4 v 13$, mel 3v8, sal 4v18, $4 v 21$ e zescaun $2 r 18,2 v 1$; e si noti l'interessante opposizione tra l'ivi attestato formaio $3 r 11$ e formay 1r5, 1r7, 1r9 [t. 5] delle note), ciò che - stante la medesima paternità delle scritture - potrà essere ricondotto alla diversa natura dei documenti (segnatamente alla maggiore informalità delle annotazioni), al basso numero di contesti fonosintatticamente favorevoli nel testo della tariffa (che in buona parte registra nomi di merci

19 In assenza di argomenti analoghi a quelli addotti da Bertoletti $(2005,116-37)$ è difficile determinare l'esatto valore di -o in questi esempi: si potrebbe trattare di forme restitutive (visto anche l'avanzamento dell'apocope di -o in Co(n)ter, formay e Peder, per cui cf. infra), ma è bene non escludere altre interpretazioni.

20 Altrimenti Iache $(m) 8 v 8,8 v 24$.

21 Altrove solo brexa(n) 3v24, 3v27, zescau(n) $2 r 20,2 v 18,2 v 20$ (t. 14) e Zeschi(n) $1 r 4$. 
isolati in contesto latino), nonché alla presenza di antroponimi locali alle cc. $1 r$ e $8 v$.

\subsection{Fonetica: consonantismo}

L'occlusiva dentale sorda in posizione intervocalica si sonorizza in fradel 8v26, 8v31, lavorada 5r7, menuda 4r20, 4v18, mesuradori 8v7, prede $4 r 24$ (si veda al termine di questo paragrafo), scudi 5r5, specificada $5 r 21$, vedelo $2 v 26,4 r 20 ;{ }^{22}$ si registra invece il dileguo della consonante nei continuatori di -ATU(M), -ATI, -ATAE, -UTU(M), -UTAE (cf. gli esempi elencati nel paragrafo precedente) e in zescaun 2 r18, $2 v 1,-(n) 2 r 20,2 v 18,2 v 20$ (t. 14), zescauna 3r21, 4r11, 4r15 (t. 9). La consonante è conservata come $t$ nei cultismi capitoli $5 r 21$ e - ammessa la bontà dell'integrazione - distin[a]to $3 v 1$; nulla di veramente utile si ricava, naturalmente, dalla forma dat. 'date' $1 r 2$, 'dati' $1 r 4,8 v 4$, 8v6. Da Petru(M) si giunge a Peder $8 v 30$ per mezzo della trafila $P e-$ dro > Pedr > Peder; analogo lo sviluppo che ha portato a Co(n)ter $1 r 11$, ipocoristico di 'Buonincontro'. Per le sorti dei nessi di dentale + vibrante segnaliamo anche il toponimo Ledro $1 r 1$.

Per il trattamento delle occlusive velari notiamo le sonorizzazioni di -C- in largà 3r24 (precedente alla sincope della vocale protonica), orbage 3v26, 4r3, 4r5, pegola $4 r 28$ (cf. anche pegule $4 r 29$ in contesto latino), pegora $4 r 7,4 r 20$. La sonora è conservata in legumi $3 v 5 \mathrm{e}$ in i(n)tregi 2v20, -o 3v11 (< INTEGRU(M) con metatesi: cf. oltre nel paragrafo); si ammutolisce ed è sostituita da un suono di passaggio in dove $3 r 6,3 r 8,3 r 10$ (cf. Rohlfs, § 217; si veda anche dovas poco oltre in contesto latino). Degna di nota la presenza di $c r$ - in crope $3 r 5 .^{23}$

$\mathrm{Da}$-P- si ha - $v$ - in civole $3 r 1$ (ma vedi la nota 11 ) e pevero $4 r 9$; identico lo sviluppo di -B- in av(er) 8v1, 8v26, cavale 2v22, -i 2v18, 2v20, $2 v 22$, lavorà $3 r 13,4 r 17$ bis (t. 5), lavorè $3 r 5$, sevo $5 r 15$, travi $3 v 1,4 v 4$ (cf. anche $A v a(n) \operatorname{cin} 8 v 16,8 v 26,8 v 29,8 v 31$ e Ava(n)cino 8v8, 8v12, da 'Avanzo'). Il nesso -PR- dà -vr- in cavra $2 v 17,4 r 20$, -e $4 r 20,{ }^{24}$ mentre si conserva nel toponimo di matrice dotta Cipri $4 v 21$. La sorda resiste in posizione intervocalica nel cultismo capitoli 5r21. Da notare ancora il participio abù 1r5, spiegabile, se vale l'interpretazione proposta da Bertoletti $(2005,253)$ per analoghe forme veronesi,

22 Cf. inoltre rivadegi $2 r 2$, - o $2 r 6,3 r 3,3 r 4$ (t. 9) in contesto latino, rilevanti anche per il trattamento delle occlusive velari e bilabiali (su cui cf. infra).

23 Per altre attestazioni con velare sorda cf. TLIO, s.v. groppa; si veda anche il § 3.5, s.v. crope.

24 È vero che le varietà semiladine del Trentino presentano oggi forme quali ['tfaưra], ['çaưra] e che nello stesso capoluogo è normale il tipo ['kaora]; non pare il caso, tuttavia, di leggere la $<u>$ presente in caura, caure del ms. come $u$, visto anche che l'Alto Garda e le valli del Sarca e del Chiese conoscono esclusivamente ['kavra] (cf. ALD-I, 1, 118). 
ipotizzando un rifacimento su un «congiuntivo aba 'abbia' analogo a deba 'debbia' (forse da *aiba, oppure rifatto sulla II pers. sing. abi)».

Da C- + vocale palatale si ha un'affricata alveodentale sorda: si vedano zente $5 r 9$, zentenaro $2 v 1$, zenture $5 r 19$; identico l'esito in posizione postconsonantica: falze $3 r 21,4 r 24 .{ }^{25}$ Si presentano con grafia conservativa, oltre a Cipri $4 v 21$, cera $2 v 10$, civole $3 r 1$ e porci $4 r 6$ (vedi § 3.1). Tra vocali la consonante passa a una sibilante sonora: lareso 3r6, 3r10, masna $3 v 15$ (dove la sincope della vocale postonica è cronologicamente posteriore all'assibilazione), noxe $3 v 21$; si ha $c$ in specificada $5 r 21$, su cui si veda ancora il § 3.1.

Registriamo qui l'antroponimo Fra(n)zolo 1r3, che adatta il consonantismo del francese frange (vedi § 3.6), e formay $1 r 5,1 r 7,1 r 9$ (5 t.), formaio 3r11, tipo su cui cf. da ultimo Bertoletti 2005, 171-2.

Per gli esiti di J si segnalano veze $4 v 15$ e Zoha(n) $1 r 10,8 v 26$, cui si affianca l'antroponimo di matrice culta Iache $(m) 8 v 8,8 v 24,-m$ 8v12; per CJ azalo 2r9, pezo 3r8; per DJ ma(n)zo 2v12, 2v24, mezani $2 v 20$, mezene 3v6 (seguito da mezena in contesto latino), mozo 3v22, 3v24, v(er)ze 4v17; per NGJ sonza 5r1, sonz(a) 8v22; per LJ ayo 2r3, fiol 1r2, foia $3 r 15$ e olio 3r10, 3v22, 3v24 (t. 6); ${ }^{26}$ per NJ Cagno(n) $1 r 10$ (vedi § 3.6), ${ }^{27} \operatorname{castag}(n) e$ 3r4, Colog(n)a $1 r 5$ (vedi § 3.6), pig(n)olà 4r12, 4r15, scalogne $5 r 17$ (qui anche ogni $2 r 16,4 r 20$, og(n)i 2v3, 3v1, 3v21 [t. 5]); per RJ canteri $4 v 4$, coramo $8 v 19,8 v 28$, cori $2 v 12,2 v 15$, presoro $4 r 22$, stora $4 v 11$, zentenaro $2 v 1$; per SJ rasa $4 v 2$; per TJ peza $4 r 15$, $4 r 16$, raxo(n) 8v29 («per sovrapposizione del suffisso -SJONEM»: Bertoletti 2005, 168); ${ }^{28}$ per xJ brexa(n) 3v24, 3v27.

Come già osservato da Magagna (1995, 292), il testo offre interessanti esempi di risoluzione dei nessi di consonante $+\mathrm{L}$, sia per la serie labiale sia per quella velare: biava $2 v 3$, fianco $3 r 13$, sechie $5 r 9$, $5 r 11$; cf. anche il germanismo schiete $5 r 11$. Il dato è notevole se confrontato con le particolari condizioni dell'Inventario giudicariese, degli Statuti dei Battuti e di altri antichi testi trentini, che presentano esclusivamente sequenze di consonante $+l$; sequenze di difficile interpretazione, anche perché, com'è noto, il Trentino ospita ancor oggi varietà che mantengono saldi i nessi di consonante $+\mathrm{L} .{ }^{29}$ All'anali-

25 Segnaliamo anche l'antroponimo Zeschi(n) 1r4 (vedi § 3.6).

26 Sugli esiti di OLEU(M) in territorio veneto, dove la conservazione di LJ appare caratteristica di veronese e padovano, cf. Bertoletti 2007, 56 nota 90; in generale cf. Rohlfs, $\S 280$. L'esito conservativo nelle forme di 'olio' è la norma negli antichi testi trentini (cf. Cristelli in preparazione); su oleo e olio nello Zibaldone del Buonconsiglio cf. già Benedetti, Brugnolo 2002, 141.

27 L'alterato, come in italiano, sarà «formato sul femm. cagna» (TLIO, s.v. cagnone).

28 Si potranno forse aggiungere Ava(n) cin $8 v 16,8 v 26,8 v 29,8 v 31, A v a(n)$ cino 8v8, $8 v 12$, da 'Avanzo' (probabilmente con $<c>=$ [ts]: vedi § 3.1).

29 Cf. ad es. Battisti 1910, 200-1; Tomasini 1955, 148 e 156-7; Bonfadini 1992, 43; vedi anche l'isoglossa 2 nella Carta dei dialetti d'Italia di Pellegrini (1977). 
si della documentazione antica, che offre esempi di risoluzione anche in altri testi quattrocenteschi (cf. già Benedetti, Brugnolo 2002, 144 e 146; Ressegotti 2012, 197-8), sarà dedicata particolare attenzione in Cristelli (in preparazione), a cui si rinvia; qui si noti, in riferimento a Riva, che la carta del 1321 edita da Stenico (2013) offre solo plovo 'misura agraria' passim, mentre esempi di palatalizzazione della L nel nesso PL sono attestati dalle suppliche tardotrecentesche - ma in copia, lo si ricorda, del primo Quattrocento - rivolte dai Tennesi e dai Rivani ad Antonio della Scala (ad es. piassa, più; sui due testi vedi la nota 1$)$.

Il nesso CT dà $t$ in fata $2 r 16,2 v 3,4 r 27,4 v 10$, zente $5 r 9$ e zenture 5r19; notevole in questo senso fruite 4 r26, che rappresenta, tuttavia, un tipo discretamente diffuso in area nord-orientale, non veramente indicativo per le sorti del nesso (sulla questione cf. Bertoletti 2005, 190). ${ }^{30}$

Per quanto riguarda la laterale, si osservano capeli 'cappelli' $2 v 27$ e cavali $2 v 18,2 v 20,2 v 22$, con trattamento conservativo di -LLİ, accanto a $q(u) a i \quad 8 v 24$, che mostra invece una significativa riduzione di

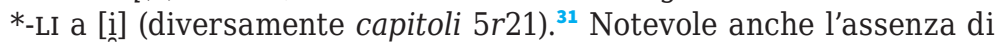
vocalizzazione in falze $3 r 21,4 r 24$, smalzo $5 r 2 .^{32}$

Tolto il caso aspecifico di no 'non' 4r17, 4r21, 5r21, la nasale alveolare in posizione d'uscita si conserva in Ava(n) cin 8v16, 8v26, 8v29, 8v31, oton $4 r 1$, zescaun $2 r 18,2 v 1$, quella bilabiale in Iachem $8 v 12$; la qualità della consonante rimane incerta nei casi di brexa(n) 3v24, 3v27,

30 Nei più antichi testi trentini la regola è cT $>t$ (cf. Cristelli in preparazione); sulle sorti del nesso in regione cf. già Battisti 1906, 174-5. Per gli esiti di cT nei testi settentrionali del Medioevo cf. Arcangeli 1990, 17-18.

31 Negli antichi testi veronesi, come in quelli di area veneto-centrale, è normale lo sviluppo -LLī > -[dzi] (begi, igi, quigi, ecc.), mentre -Lī è tendenzialmente conservato; le aree lombarda e veneto-settentrionale rispondono invece con la riduzione a [i] tanto di -LLì quanto di -Lī (fradey, quey, ecc., ma anche animay, fioy, ecc.): in generale, si vedano le considerazioni di Bertoletti (2005, 180-2), che pure segnala, per Verona, isolati fenomeni di palatalizzazione (anche con esito -i) di -Lİ e *-LI. Mette conto osservare, in questo senso, che l'Inventario giudicariese reca botexey 37 , gay 28,34 , ecc. allato a faxoy 26, linzoy 24, ecc., mentre gli Statuti dei Battuti attestano solo la riduzione di -LLİ (ad es. fradey 16, 20, 22 e passim, quey 18, 22, ma lençoli 38); Benedetti, Brugnolo $(2002,143)$ registrano, nello Zibaldone del Buonconsiglio, "sempre li non gi, queli non quigi, ecc.», ma cavegni 'capelli' (con <gn> = [d]]?); le suppliche ad Antonio della Scala (vedi la nota 1) presentano forme palatalizzate di tipo veronese (igi, quigi); per questi dati e per altre osservazioni si rinvia a Cristelli (in preparazione). Si noti che per il pronome ge $8 v 30,8 v 31$ (vedi § 3.4 ) non è necessario supporre un esito palatalizzato di -LLİ ([d]e] < ILLĪ), dato che la forma può essere letta con un'occlusiva velare ed essere riportata a HIC (cf. Rohlfs, § 459).

32 Per le sorti di L preconsonantica cf. in generale Tuttle 1991; si ricorda che la vocalizzazione (con eventuali ulteriori sviluppi) è ancora caratteristica di alcune varietà del Trentino (cf. Battisti 1910, 201; Bonfadini 1989, 49-50; 1992, 43). Da notare in questo senso autra 28, fauz 40, ecc. (però smalz 42, smalzarola 37) nell'Inventario giudicariese e autramenter 'altrimenti' nel quaderno scolastico attribuito a Nicolò di Castel Campo (sec. XV in.; ed. Zingerle 1900, 392). 
Cagno(n) 1r10, castro(n) 2v17, 4r20, Iache(m) 8v8, 8v24, monto(n) 4r20, No(n) 8v12, raxo(n) 8v29, zescau(n) 2r18, 2v18, 2v20 (t. 14), Zeschi(n) $1 r 4$, Zoha(n) 1r10, 8v26, che pure permettono di osservare l'assenza di fenomeni di dileguo in posizione finale. Nulla da notare quanto alle nasali preconsonantiche, sempre conservate e, dove esplicitamente rappresentate, prive di alterazioni rispetto all'etimo latino. ${ }^{33}$

Per gli esiti della labiovelare si possono indicare solo $q(u) a i$ 8v24, $q(u e) l 1 r 6$ e, con l'attesa riduzione a [k], che 8v30, ch(e) 8v31 (relativi).

Si ha sempre conservazione di s-, anche davanti a vocale anteriore (si noti in particolare sia in osia $3 v 15) .{ }^{34} \mathrm{Ad}$ interno di parola -(N)S- dà una sibilante sonora (per le grafie $\langle\mathrm{s}\rangle,\langle\mathrm{ss}\rangle,\langle\mathrm{x}\rangle$ vedi § 3.1): asena 2r7, -o 2r7, mesuradori 8v7, pesso 'peso' 1r8, 1r10, 1 r11 (t. 5), pexo 'id.' 3r24, presoro 4r22, v(er)oneso 3v22, 3v27. Da -PS- e -ss- si ha [s]: case 'casse' 3v1, grosa 4v21, -i 2v18.

In posizione intervocalica -X- dà una sibilante sorda in asse $2 r 13$, 2r14, 2 r15 (t. 6; cf. LEI, 3, 2724); lo stesso esito in posizione iniziale secondaria: sonza $5 r 1$, sonz(a) $8 v 22$. Si registra grafia etimologica in exir $4 v 13$, dove $<x>$ varrà sicuramente [s], e in Alexandria $4 v 21$.

Un solo esempio per gli esiti di sc davanti a vocale palatale: pesso 'pesce' 4r13, con sibilante sorda.

Segnaliamo in questa sede, infine, la metatesi di $r$ in i(n)tregi $2 v 20$, -o 3v11, fenomeno ben diffuso nelle varietà settentrionali (cf. Rohlfs, $\S 322$, cui si aggiungano, ad es., Ghinassi [1965] 2006, 79; Stussi 1965, LIX; Tomasin 2004, 159); prede 'pietre' 4 r24 dipende dal tipo *PRETA, su cui cf. Aebischer 1943.

\subsection{Morfologia}

Per quanto riguarda la morfologia nominale, si consideri anzitutto la forma pevero $4 r 9$, che muove dall'accusativo *PIPERE(M) come in genere nei volgari settentrionali (cf. Bocchi 1991, 81 nota 117; Bertoletti 2005, 203).

I sostantivi di I declinazione escono in - $a$ al singolare e in - $e$ al plurale: aste 2r11, cavra 2v17, 4r20, -e 4r20, erbe 4v17, lana 2v27, 3r25, 4r16, stora $4 v 11$, vache $2 v 24,4 v 13$, zenture $5 r 19$, ecc.; apparterrà al gruppo, proseguendo TINA(M), anche il plurale tine 3r10 (cf. Bertoletti 2005, 204

33 Il testo, quindi, non documenta né il dileguo di - $n$, tratto ben diffuso nel bresciano antico (cf. Bertoletti 2001, 241), né fenomeni quali quelli descritti dallo stesso Bertoletti $(2005,182-5)$ per il veronese (in particolare $-n>-m$ e l'assai più raro $-m>-n ; n>$ $m$ in posizione preconsonantica). Per il trattamento della nasale (preconsonantica e in sede finale) nelle vallate del Trentino odierno cf. Bonfadini 1992, 39-40.

34 Per la presenza di $s c<\mathrm{s}$ davanti a vocale anteriore nei testi antichi di Verona e del resto dell'Italia settentrionale - per l'appunto anche in casi come scia, sciando, ecc. - e per le possibili spiegazioni del fenomeno cf. invece Bertoletti 2005, 187 e nota 468. 
nota 518; per tina nei dialetti moderni del Trentino cf. Pedrotti 1936, 65). Foia $3 r 15$ e pegora 4r7, 4r20 sono forme di neutro plurale rianalizzate come femminili singolari di I declinazione; si registra al proposito, per quanto attestato solo al plurale, anche fruite 4 r26 (cf. infra).

I sostantivi di II declinazione escono in - $o$ al singolare e in - $i$ al plurale: aseno $2 r 7$, canteri $4 v 4$, cavali $2 v 18,2 v 20,2 v 22$, ma(n)zo $2 v 12$, $2 v 24$, molino 3v17, porco 3v6, -i 4r6, saco 3v21, 4r26, 4v1, vedelo $2 v 26$, $4 r 20$, ecc. Non sono attestate forme plurali in -a ed -e (carra, brazze, ecc., su cui cf. Loporcaro 2018, 208-11).

Quando non presentano apocope della vocale finale, i sostantivi di III declinazione escono al singolare in -e (maschili: legname 2r16, pelame 4r17, 4r20; femminili: barile 4r28, carne 2v24, 2v26, falze 4r24, nave $3 v 1$, pele $4 r 20,-l l-4 r 21$ ); resta incerto se nei casi di azalo $2 r 9$, coloro $4 v 8$, coramo $8 v 19$, $8 v 28$ e di altri maschili in -o si debba pensare agli effetti di un fenomeno di natura morfologica o fonetica (vedi § 3.2). Al plurale le uscite sono -i (maschili: boy 2r20, 2v24, legumi 3v5, mesuradori $8 v 7$, travi $3 v 1,4 v 4$ ) ed -e (femminili: asse 2r13, 2r14, $2 r 15$ [t. 6], falze 3r21, noxe 3v21, pelle $8 v 30)^{35}$

Per la IV declinazione si noti fruite 4 r26, per cui si deve pensare a un passaggio alla II declinazione come neutro e a una successiva rianalisi del plurale in -A come singolare femminile (cf. l'analogo caso di frute commentato in Bertoletti 2005, 204 e 209).

Dalla v declinazione il singolare dì $8 v 17$.

Quanto al genere si notino, oltre a peri 4 r26 (cf. Rohlfs, § 382), asse femminile (asse longe $2 r 14$, asse pizole 2r15) e travi maschile (ai travi $3 v 1$ ), atteso in quest'area come sal femminile (sal menuda $4 v 18$, sal grosa $4 v 21$; inoltre la sal segnalato in apparato nel $\S 2) ;{ }^{36}$ notevole de zescauna barile $4 r 28$, che trova un riscontro in [la ba'ri:l] attestato dall'AIS, 8, 1325 a Mortaso (punto 330). ${ }^{37}$ È maschile dì 8v17 (dì s(upra)s(crip)to).

Gli aggettivi di I classe presentano paradigmi uscenti in -o/-i (maschile) e -a/-e (femminile); più interessante il metaplasmo documentato dall'aggettivo di II classe gra(n)da 3v17, tipo assai diffuso nei volgari settentrionali (cf. Rohlfs, § 396). Di origine metaplastica - più che dovuta agli sviluppi di -e finale (vedi § 3.2) - sarà la -o di v(er)oneso $3 v 22,3 v 27$.

35 Naturalmente, non si può escludere che asse e noxe rappresentino sostantivi passati alla I classe, visto il comportamento delle varietà settentrionali antiche e moderne (per Verona cf. Bertoletti 2005, 204-5); si noti ad ogni modo che i dialetti trentini attestano solo raramente (e solo in area orientale) il tipo nosa (cf. ALD-I, 3, 512), mai il tipo assa (cf. LEI, 3, 2717-19).

36 Per il genere assunto dai continuatori di $\mathrm{AXE}(\mathrm{M})$ nelle varietà italoromanze si veda LEI, 3, 2710 ss. Per travi e sal cf. Bertoletti 2005, 212 e l'ivi citato Rohlfs, §§ 385 e 394

37 Si consideri anche, in contesto latino, de q(u)alib(et) barile 4r29. Pro qualibet barille è attestato due volte nella tariffa della muta del 1370 (cf. Malossini 2003, 161). 
Per le forme dell'articolo si registrano i determinativi el (tre volte in contesto distributivo: $p$ (ro) IIJ libr. el pesso $1 r 8,1 r 10,1 r 11$; inoltre ch(e) ge dè el fradel 8v31), i (r. XIJ duc. i q(u)ay 8v24), la (la sal, per cui cf. l'apparato dell'edizione di c. $8 v$ nel $\S 2$ ); quanto ai continuatori di UNU(M), ha valore chiaramente numerale l'unico esempio utile: cori de ma(n)zo d(e) uno a(n)no 2v12. Preposizioni articolate: al fiol $1 r 2$, al Co(n)ter $1 r 11$, ai travi 3v1, ai mesuradori $8 v 7$, ala soa $8 v 29$, ale case 3v1, del Ligo 8v25, d(e)la Val 8v12; notevole i(n)di 'nei' in i(n)di s(upra)s(crip)ti capitoli $5 r 21 .^{38}$

Per la serie dei pronomi si segnala la forma obliqua atona di III pers. sg. ge in che ge dè $8 v 30$, ch(e) ge dè $8 v 31$ 'che gli diede'. I contesti citati, come si vede, documentano anche il relativo che, con funzione di oggetto come nel caso di i $q(u) a i$ 8v24. Quanto ai dimostrativi, si registra q(ue)l in XLV libr. d(e) formay d(e) q(ue)l d(e) Ma(r)tino $1 r 6$; per gli indefiniti si notano l'invariabile ogni $2 r 16,4 r 20, o g(n) i$ $2 v 3,3 v 1,3 v 21$ (t. 5) ${ }^{39}$ e zescaun 'ciascuno' $2 r 18,2 v 1,-(n) 2 r 20,2 v 18$, $2 v 20$ (t. 14), zescauna $3 r 21,4 r 11,4 r 15$ (t. 9); per i possessivi si trova solo soa in ala soa raxo(n) 8v29.

Quanto alla morfologia verbale, tolti i participi passati deboli già registrati nel $\S 3.2$ s'incontrano solo à 'ha' $1 r 5,1 r 7,1 r 9,8 v 24, d\left(e^{\prime}\right)$ 'deve, devono' 1r1, 1r3, 8v8, 8v12, de' 'deve' 8v1, 8v26 bis, 8v28, dè 'diede' $8 v 30,8 v 31$, resta $8 v 11$ (resta XVIIJ duc.), gli infiniti amolaro $3 v 19$ e molaro $3 v 15$, av(er) $8 v 1,8 v 26$, dar $8 v 26$, daro $1 r 1,1 r 3,8 v 8$ (t. 5), exir $4 v 13$ e le forme di natura participiale distin[a]to $3 v 1$, lavorada $5 r 7$, specificada $5 r 21$ e zente $5 r 9$; si può registrare anche il congiuntivo sia in osia $3 v 15$.

38 Sul tipo indel cf. la bibliografia citata in Bertoletti 2005, 218 nota 551. Per la presenza di indel (e intel) nello Zibaldone del Buonconsiglio cf. Benedetti, Brugnolo 2002, 144

39 Sul rapporto tra gli invariabili ogna e ogni a Verona e più in generale sulle condizioni dei volgari di area lombarda e veneta si veda Bertoletti 2007, 59 e nota 99. I testi trentini considerati in Cristelli (in preparazione) recano sia ogna (invariabile) sia ogni. 


\subsection{Lessico}

Si propone di séguito una schedatura pressoché integrale delle forme volgari offerte dal testo. ${ }^{40}$ Le voci più interessanti sono dotate di approfondimenti volti a chiarirne la natura e a fornire riscontri utili da fonti latine e volgari, nonché dai dialetti moderni; uno spazio particolare è stato destinato ai confronti con altri tariffari relativi alla muta rivana. ${ }^{41}$ In tutti i casi in cui la parola si trova in un brano volgare, considerato anche che della tariffa non si è data una trascrizione completa, è sembrato opportuno riprodurre in tutto o in parte il contesto (la voce in esame è abbreviata alla sola iniziale). Le forme in esponente sono ordinate alfabeticamente senza distinguere tra $i$ e $y, s$ e $x$ e trattando le doppie come scempie. A lemma, nel caso di varianti, si inserisce il tipo più frequente o il primo attestato là dove si contino pari occorrenze. I sostantivi compaiono al singolare, gli aggettivi al maschile singolare, i verbi all'infinito; in assenza di tali forme si lemmatizzano i sostantivi e gli aggettivi così come sono attestati (al plurale o al femminile), mentre si ricostruisce l'infinito dei verbi ponendolo tra parentesi quadre. ${ }^{42}$ A differenza di quanto fatto sinora, non si dà mai conto dello scioglimento dei compendi; si accentano invece tutti i lemmi sdruccioli e terminanti in -ìa. ${ }^{43}$ Per i rinvii interni al glossario si ricorre al simbolo $\rightarrow$.

40 Sono esclusi gli articoli e le forme articolate, i pronomi, le voci dei verbi 'avere', 'dare', 'dovere' e poche altre forme ritenute di scarso interesse.

41 Si farà riferimento, nella fattispecie, alla tariffa del 1370 (in latino; cf. Malossini 2003, 160-3) e a quelle volgari del secondo Quattrocento pubblicate e glossate da Sardagna (1884). Trascuriamo i lavori di Reich (1903) - traduzione italiana di una tariffa in latino del 1426 - e Martinelli (2000), dove si ripropone un documento già noto al Sardagna attribuendolo per errore al 1458 (e non al 1490) e traendolo forse - come risulta da alcuni elementi emersi durante un colloquio con la dottoressa Anita Malossini (Archivio comunale di Riva) - da una copia più tarda.

42 Dato che il testo offre, in forma piena, solo infiniti uscenti in - 0 , alle forme ricostruite è assegnata la stessa terminazione.

43 Nel caso di ingesto, -e l'accentazione resta incerta. 
Stefano Cristelli, Mario Wild

Il volgare trentino in una tariffa rivana del 1409

agnelo: pelamede [...] a. 4r20.

ayo 'aglio' $2 r 3$.

amolaro: mola pizola da a. pro exitu - $v$ s. veron. $3 v 19$. La voce, vista anche la menzione della mola pizola (cf. già Sardagna 1884,414$)$, non coinciderà semanticamente con $\rightarrow$ molaro e indicherà invece l'atto di 'affilare' (cf. TLIO, s.v. ammolare [1]).

asse 'assi' $2 r 13,2 r 17$; a. longe $2 r 14$; a. pizole 2r15; a. e legname de ogni fata 2r16; a. de zescaun caro [...] viIJ s. veron. $2 r 18$.

àsena 'asina' $2 r 7$.

àseno 'asino' $2 r 7$.

aste $2 r 11$.

azalo 'acciaio' 2r9. Da ACIĀLE(M). Cf. LEI, 1, 408 ss.; TLIO, s.v. acciale.

barile: pegola de zescaunab. [...] J s. veron. 4 r28.

becho 'becco, caprone' $2 v 8$.

biava 'biada': $b$. de ogni fata $2 v 3$.

bo' 'bue': boy de zescaun b. [...] IJ s. veron. 2r20; pl. boy: oltre al contesto appena citato, cf. carne de b., de vache e de manzo $2 v 24$.

brexan 'bresciano' (detto dell'olio proveniente dalla sponda bresciana del Garda): olio b. de zescaun mozo [...] xxvJ s. VIIJ dr. veron. 3v24; olio suprascripto veroneso e b. e lorino $3 v 27$.

bronzo: b. de zescaun zentenaro [...] vs. veron. $2 v 1$.

canteri 'travi di sostegno': travi e c. 4v4. Da CANTHĒRIU(M). Cf. LEI, 10, 1425 sS.; sul tipo cf. anche Bertoletti 2005, 460. Si noti cantèri in Sardagna 1884, 410.

capeli 'cappelli, copricapi': c. de lana e de fero $2 v 27$. Si veda in generale TLIO, s.v. cappello; cf. inoltre capelo de fero 'tipo di elmo' in Bertoletti 2005, 460.

capitoli: zescauna soma de mercandaria no specificada indi suprascripti c. $5 r 21$.

carne: c. de boy, de vache e de manzo $2 v 24 ; c$. de vedelo 2 v26.

caro 'carro' (come unità di misura di volume): asse de zescaun c. [...] viIJ s. veron. 2r18; vino de ogni fata de zescaun c. pro exitu - vIIJ s. ver. 4v10; sal menuda [...] de zescaun c. J libr. x s. ver. 4v18. Cf. TLIO,
S.v. carro, 2; sul carro come unità di misura per il vino a Trento (pari a 628 litri) cf. Curzel, Gentilini, Varanini 2004, 86.

case 'casse': legnamo distin[a] to ai travi e ale c. de ogni nave $3 \mathrm{v} 1$.

castagne $3 r 4$.

castron 2v17; pelamede [...]c. 4r20.

cavale 'cavalle': cavali ingeste e c. pro exitu de zescaun - vs. veron. $2 v 22$.

cavali 'cavalli': c. grosi $2 v 18$; c. intregi mezani $2 v 20$ (cf. $\rightarrow$ intrego e mezani); c. ingeste e cavale pro exitu de zescaun - v s. veron. 2v22 (cf. $\rightarrow$ ingesto).

cavra 'capra' 2v17; pelame de monton, c., cavre 4r20; pl. cavre (solo nel contesto appena citato).

cera $2 v 10$.

civole 'cipolle' $3 r 1$. Per l'accentazione piana si veda la nota 11.

coloro 'colore': tera de c. 4v8. Cf. $\rightarrow$ tera.

[compraro]: part. pass.: comprà $8 \mathrm{v} 29$ (pro resto de coramo c. da Avancin).

coramo 'cuoio': r. LxxJ libr. perc. vendù a Ventura da $\mathrm{Gar}^{\circ}$ 8v20; pro resto de c. comprà da Avancin 8 v29. Cf. TLIO, s.v. cuoiame; Tomasin 2004, 246.

cori 'cuoi': c. de manzo de uno anno 2 v12; c. de Garzaria e nostrani 2v15. Su Garzaria vedi §3.6.

crope 'groppe', con riferimento alle pelli ricavate dal dorso degli animali: c. lavorè $3 r 5$. Nell'Inventario giudicariese si trova la terza part d'una cropa 39. Cf. SellaE, s.v. croppa 'il cuoio della groppa delle bestie' (Modena, 1327); Sella, s.v. cropa 'il dorso della bestia, la groppa' (Venezia, 1271; Verona, 1318). Cropa laborata è anche nella tariffa del 1370 (cf. Malossini 2003, 162); si aggiunga cròpe de bò in Sardagna 1884, 411, che glossa con «cuojo della groppa del bue» e attribuisce la voce al «dialetto bresciano e trentino». Per le varietà della Lombardia orientale cf. Melchiori, s.v. cropa 'cuoio' e 'groppa'; Tiraboschi, s.vv. cropa 'groppa' e cropa de cöràm 'cuoio'; per il Trentino cf. ad es. Quaresima, s.v. cròpa, gròpa.

cugnà 'cognato': Martino c. de Martinel $1 r 6$. 
distin[a]to: legnamo d. ai travi e ale case de ogninave - $\mathrm{xx}$ s. veron. $3 \mathrm{v1}$. Forma ricostruita; il manoscritto ha distinto.

dove 'doghe': $d$. de lareso $3 r 6$; $d$. de pezo $3 r 8$; d. de lareso da tine da olio $3 r 10$.

erbe: verze e e. $4 \mathrm{v} 17$.

exir 'uscire': vache per e. de zescauna- J s. ver. $4 v 13$. Per exir corrisponde al lat. pro exitu, frequentemente impiegato nel testo con riferimento alla tassazione sulle merci in uscita.

falze 'falce': prede da f. $4 \mathrm{r} 24$ (cf. $\rightarrow$ prede); pl. falze: $f$. de zescauna soma [...] x s. veron. $3 r 21$.

fata 'fatta, sorta, tipo': asse e legname de ogni f. 2r16; biava de ogni f. 2v3; pomi de ogni f. 4227 ; vino de ogni f. $4 v 10$.

fero 'ferro' 3r17; xxvII libr. de f. 1r2; capeli de lana e de f. 2v27; f. scartà $3 r 19$ (cf. $\rightarrow$ scartà); sechie zente de f. $5 r 9$.

fianco: f. lavorà 3r13. È la pelle del fianco dell'animale: cf. ad es. Sella, s.v. flancus; SellaE, s.vv. fianchus e flanchus. Per Riva cf. fla $[n]$ cho nella tariffa della muta del 1370 (cf. Malossini 2003, 162); si vedano inoltre fianco e fianchi nelle tariffe del secondo Quattrocento (cf. Sardagna 1884, 398 e 405).

fiol 'figlio': xxvis libr. de fero dat. al $f$. $1 r 2$.

foia 'foglia': f. de roso 3r15. Cf. $\rightarrow$ roso. Da notare che il veronese antico attesta il solo foia 'foglia di sostanza impiegata nella concia' (cf. Bertoletti 2005, 478); nei dialetti trentini sono sopravvissute, con il valore di 'scotano', forme quali foiaròla (e varianti) e foiól, «quasi ad indicare la foglia per eccellenza, quella che i nostri contadini una volta diligentemente raccoglievano per la concia delle pelli» (Pedrotti, Bertoldi 1930, 328).

formay 'formaggio': xLV libr. de f. 1r5; xxVIIJ $\div$ libr. de f. 1 r 7 ; xu libr. de f. 1r9; xxV libr. de f. 1r10; xLIIIJ libr. de f. 1r11; formaio: $f$. de zescaun pesso [...] IIIJ dr. veron. $3 r 11$.

fradel 'fratello': Zohan f. de Avancin 8v26; elf. de Avancin 8v31.

fruite 'frutti': peri ef. 4 r26.

granda 'grande': mola g. da molino $3 v 17$.

grosi 'grossi': cavalig. 2v18; grosa, con riferimento al sale (contrapposto a $\rightarrow$ menuda): sal g. de Ciprio de Alexandria $4 v 21$.

ingesto 'castrato' (detto di muli e cavalli): mulo i. e mula $3 \mathrm{v} 13$; pl. ingeste (probabile errore per ingesti): cavali i. e cavale 2v22. Da confrontare con il medio-alto-tedesco heng(e)st 'cavallo castrato'; in tedesco moderno il significato della voce è quello opposto di 'stallone' (documentato a partire dall'inizio del sec. XV: cf. DW, 10, 986; EWD, s.v. Hengst), ma la semantica medio-alto-tedesca è conservata in Baviera (cf. Schmeller, 2, 214) e in varietà tirolesi (cf. Schatz, s.v. hengest). Quanto alla presenza del tipo nei volgari italoromanzi, cf. Mussafia 1873,53 , che documenta il sostantivo engiosto - glossato per l'appunto con hengst - in un vocabolario veneto-tedesco del sec. XV («ein deutsches Wort, das ich sonst nicht nachweisen kann»); all'attestazione, su cui cf. anche Salvioni 1914, 579-80 nota 4 e 1916, 1019, va associato engesto in un glossario appartenente alla stessa famiglia di quello studiato da Mussafia (cf. Höybye 1974, 191). Il lessema è presente nel tariffario del 1370 (pro quolibet equo ingesto et equa; cf. Malossini 2003, 160). Un riscontro utile anche in Sella, s.v. engistus (equum magnum de armis et equum engistum; la forma, non compresa, è datata Treviso, a. 1339).

intrego 'intero', detto del mulo e del cavallo atti alla riproduzione (in quanto non castrati): mulo i. $3 \mathrm{v} 11$; intregi: cavali i. mezani $2 v 20$. Per intero 'che non ha subito castrazione' cf. GDLI, s.v. intéro, 12. Da notare equo integro, mulo integro nella tariffa della muta del 1370 (cf. Malossini 2003, 160 e 163) e cavalli integri in uno dei testi editi da Sardagna (1884, 405); si consideri anche caval intrego, chaval intrego in un documento relativo al dazio del Tonale del 1460-1461 (cf. Stenico 1979, 53, 59, 60, 73).

lana 3r25; capelidel. e de fero 2v27; panno de l. de s peza [...] J s. t. $4 r 16$.

lardo $3 r 23$.

làreso 'larice': dove de l.3r6; dove de l. da tine da olio $3 r 10$. Sul tipo in Italia 
settentrionale si veda Pellegrini 1976, 621-2. Alcuni dati relativi all'area trentina - antica e moderna - sono raccolti in Bertoletti 2005, 482-3.

largà 'resina di larice': l. de zescaun pexo pro exitu - IIIJ dr. veron. 3r24. Da LARǏCĀTU(M), sulla cui presenza in Italia settentrionale cf. Pellegrini 1976, 622. L'attestazione trova un riscontro antico nel largado presente nella Legenda di glorioxi apostoli misier sen Piero e misier sen Polo (cf. Brusegan Flavel 2005, 93, che glossa con 'bitume'); cf. anche largao in Mussafia 1873, 72. Per Riva cf. largati nella tariffa della muta del 1370 (cf. Malossini 2003, 163); largado in Sardagna 1884, 412.

lavorà 'lavorato, trattato' (detto di pellame): fianco l. 3r13; pelame l. e no l. 4r17; pelame [...] l. e no l. 4 r21 (se in questo caso non si tratta di 'lavorata': vedi la nota 12); lavorada: spala l. 5r7; lavorè crope l. $3 r 5$.

legname: asse e l. de ogni fata $2 r 16$; legnamo: l. distin[a] to ai travi e ale case de ogni nave - $\mathrm{xx}$ s. veron. $3 \mathrm{v} 1$.

legno: sechie de l. schiete $5 r 11$.

legumi $3 v 5$.

lino $3 v 3$.

longe 'lunghe': asse l. 2 r14.

lorino 'laurino, di alloro' (detto dell'olio ricavato dalla pianta): olio suprascripto veroneso e brexan e l. $3 \mathrm{v} 27$. Nel testo anche olio de orbage (cf. $\rightarrow$ orbage).

manzo: coride m. de uno anno 2v12; carne de boy, de vache e de m. 2 v24.

masna 'macina': mola da molaro osia $m$. $3 v 15$.

mel 'miele' $3 v 8$.

menuda 'minuta', detto di pelle di animale di dimensioni medio-piccole: pelame de monton, cavra, cavre, pegora, castron, agnelo, vedelo e de ogni pele m. 4r20; detto del sale (contrapposto a grosa, per cui cf. $\rightarrow$ grosi): sal m. [...] de zescaun caro J libr. x s. ver. $4 v 18$. Cf. pro quolibet plaustro salis minuti nella tariffa della muta del 1370 (Malossini 2003, 160).

mercandarìa 'mercanzia':zescauna soma de m. no specificada indi suprascripticapitoli 5r21. Cf. TLIO, s.v. mercanteria. mesuradori 'misuratori': item IIIJ s. dat. aim. $8 v 7$.

mezani 'di medie dimensioni' (detto di cavalli): cavali intregi m. 2v20. Che il riferimento sia alle dimensioni e non, per es., all'età degli animali pare confermato dalla presenza di cavali grosi $2 v 18$.

mezene 'tagli di carne suina corrispondenti alla metà della bestia' (forse con riferimento al solo lardo): $m$. de porco 3v6. Da *MĔDIĒNA (REW, 5460). Cf.j mezen de porch 31 nell'Inventario giudicariese; inoltre pro qualibet mezena nella tariffa della muta del 1370 (cf. Malossini 2003, 161) e mezène registrato in Sardagna 1884,413 . La voce è ben attestata nei dialetti di area trentina: cf. ad es. Quaresima, s.v. mezéna; Ricci, s.v. meżena; Salvadori, s.v. mezéna.

mola 'macina': m. da molaro osia masna 3v15; m. granda da molino 3v17; 'mola per affilatura': $m$. pizola da amolaro 3v19. Cf. $\rightarrow$ amolaro, molaro e masna.

molaro 'macinare': mola da m. osia ma sna 3v15. Cf. TLIO, s.v. molare (2). La lettura d'amolaro (cf. $\rightarrow$ amolaro) è da escludere per la chiara presenza di uno spazio tra preposizione e verbo.

molino: molagrandada m. $3 \mathrm{v} 17$.

monton: pelame de m. $4 r 20$.

mozo 'moggio' (unità di misura per l'olio): olio veroneso de zescaun $m$. [...] xx dr. veron. 3v22; olio brexan de zescaun $m$. [...] XXVJ s. VIIJ dr. veron. 3v24. Sul valore del moggio nella Trento medievale (circa 170 litri) cf. Curzel, Gentilini, Varanini $2004,86$.

mula: mulo ingesto e m. $3 v 13$.

mulo: $m$. intrego $3 v 11 ; m$. ingesto e mula $3 \mathrm{v} 13$ (cf. $\rightarrow$ intrego e ingesto).

nave: legnamo distin[a]to ai travi e ale case de ogni $n$. - xx s. veron. $3 v 1$.

noxe 'noci': n. [...] de ogni saco pugno $J$ $3 v 21$.

nostrani: cori de Garzaria e n. 2v15. Cf. la scheda relativa a Garzaria nel § 3.6.

nove 'nuove': veze $n .4 v 15$.

olio: cf. i contesti citati in riferimento a $\rightarrow$ brexan, lorino, orbage e veroneso, cui si aggiunga il seguente: $x J$ mod. e vis gal. d'o. $8 v 9$. 
orbage 'bacche dell'alloro' $4 r 3,4 r 5$; olio de o. 3v26. Da LAURI BACCA con discrezione dell'articolo. Documentazione antica e moderna in LEl, 4, $113 \mathrm{ss}$. Accanto a olio de 0 . il testo reca olio lorino: $\mathrm{cf} \rightarrow$ lorino.

oton 'ottone' $4 r 1$.

panno $4 r 11 ; p$. de lana de s peza [...] J s. t. $4 r 16$.

pégola 'pece': p. de zescauna barile [...] J s. veron. $4 r 28$.

pégora 'pecora' $4 r 7$; pelame de [...] $p$. $4 r 20$.

pelame 'pellame': $p$. lavorà e no lavorà $4 r 17$; p. de monton, cavra, cavre, pegora, castron, agnelo, vedelo $4 r 20$.

pele 'pelle': de ogni p. menuda $4 r 20$ (cf. $\rightarrow$ menuda); pelle: de zescauna $p .-\mathrm{J} d r$. 4r21; pl. pelle: item IIIJ libr. v s. pro is p. che ge dè Peder de Nicolò da Brezo $8 v 30$.

peri 'pere': $p$. e fruite $4 r 26$.

pesso $^{1}$ 'peso' (come unità di misura): pro IIJ libr. el p. 1r8, 1r10, 1r11 (sempre in riferimento al formaggio); formaio de zescaun p. pro exitu - IIIJ dr. veron. 3r11; presoro de zescaun $p$. pro exitu - IIIJ dr. veron. 4r22; pexo: largà de zescaun $p$. pro exitu - IIIJ dr. veron. 3r24. Per il Trentino cf. ad es. Schneller 1898, 153.

pesso $^{2}$ 'pesce' $4 r 13$.

pévero 'pepe' $4 r 9$.

peza 'ritaglio di stoffa' (come unità di misura): pignolà de zescauna $p$. [...] vJ dr. 4r15; panno de lana de J p. [...] J s. t. 4r16. Cf. TLIO, s.v. pezza, 2.4 .

pezo 'abete rosso': dove de p. 3r8. Da *PǏCEU(M). Cf. Bertoletti 2005, 495 e, per la diffusione moderna della voce, l'ivi citato Pellegrini 1976, 614-16.

pignolà 'stoffa ricamata con motivi simili a pinoli' $4 r 12 ; p$. de zescauna peza [...] vJ dr. $4 r 15$. Sul tipo si veda il materiale raccolto in Bertoletti 2005, 495-6. Cf. pro qualibet petia pig[n]olati e pro qualibet soma pig[n]olati nella tariffa della muta del 1370 (Malossini 2003, 161); cf. inoltre pignolado in Sardagna 1884, 415. Per la voce in Trentino si considerino ancora Quaresima e Ricci, s.v. pignolà. pìzola 'piccola': mola $p$. da amolaro $3 v 19$; pl. pizole: asse p. $2 r 15$.

pomi: p. de ognifata $4 r 27$.

porco: mezene de p. $3 v 6$; pl. porci $4 r 6$.

prede 'pietre': in $p$. da falze 'pietre per affilare la falce, coti' 4 r24. Cf. pro qualibet soma lapidum prede laboratarum a falcis nella tariffa della muta del 1370 (Malossini 2003, 162) e prede da fauz 40 nell'Inventario giudicariese.

presoro 'caglio, presame': $p$. de zescaun pesso pro exitu - IIIJ dr. veron. 4r22. Si ricostruisce *PRENSŌRIU(M): cf., per il Trentino, Rosalio 1979, 139 e Tomasini 1990, 157; più in generale cf. Plomteux, s.v. presū, e l'ivi citato AIS, 6, 1212. Per attestazioni mediolatine cf. Du Cange, s.v. praesorium (2), e specialmente presorii nella tariffa della muta del 1370 (cf. Malossini 2003, 162). Cf. anche presór registrato in Sardagna 1884, 416.

[prestaro]: part. pass.: prestay 1 r3 (Franzolo de'daro - XIIIJ libr. XVIIJ $\div$ s. perd. p.).

pugno (come unità di misura per la frutta): de ogni saco p. J 3v21; de zescaun saco s p. $4 r 26,4 v 1$.

ramo 'rame' $4 r 30$.

ranzi: r. [...] de zescaun saco s pugno $4 v 1$. Da chiarire: il fatto che per misurare la merce - com'è per le castagne, le mele, le pere, le noci e in generale le fruite - ci si riferisca a sacchi e pugni lascia pensare alle arance (dialetto trentino moderno narànzi; cf. ad es. Ricci, s.v. narànz); ma andrebbe approfondito il problema del commercio degli agrumi in area gardesana nel primo Quattrocento. Per 'arancia' in antico cf. TLIO, s.Vv. arancia e arancio.

rasa 'resina di abete' $4 v 2$. Per * $R A S I A$ in Trentino e nel resto dell'Italia settentrionale cf. Pellegrini 1976, 612-13.

raxon 'ragione': in segnà ala soa r. 'registrato a suo debito' 8 v29.

[restaro]: ind. pres.: III pers. resta $8 \mathrm{v} 11$ (r. XVIIJ duc.... J gros.).

resto 'rimanente': de' dar Zohan fradel de Avancin xxxs libr. per r. de $v$ mod. olei 8v27; cf. anche: Ventura da $\mathrm{Gar}^{\circ} \mathrm{de}$ ' $d a-$ ro xxvis libr. pro r. de coramo comprà da Avancin e segnà ala soa raxon 8v29. 
roso 'sommacco (Rhus coriaria L.)' o 'scotano (Rhus cotinus L.)' (impiegato come sostanza colorante nella concia): foia de r. 3r15. Da Rōs. Informazioni sul tipo e attestazioni mediolatine in Bosshard 1938, 232-4; cf. anche foliam rosii nello statuto veronese del Misterium Solarolorum del 1319 (citato in Bertoletti 2005, 478). Per Riva cf. pro quolibet modio folei rosi nella tariffa della muta del 1370 (cf. Malossini 2003, 161); inoltre foia de roso e legno de roso registrati in Sardagna 1884, 411. Continuatori dialettali di area trentina sono segnalati in Pedrotti, Bertoldi 1930, 328.

saco 'sacco' (come unità di misura per la frutta): de ogni s. 3v21; de zescaun s. $4 r 26,4 v 1$.

sal 'sale': s. menuda [...] de zescaun caro $\mathrm{u}$ libr.x s. ver. $4 v 18$; s. grosa de Ciprio de Alexandria 4v21; xvs gal. de s. 8v2; cf. inoltre la sal segnalato nell'apparato dell'edizione di c. $8 v(\$ 2)$. Per altre attestazioni di 'sale di Cipro' si veda DI, 1 , 514.

scalogne 'scalogni' 5r17. Cf. LEI, 3, 1528 ss.; TLIO, s.v. scalogna. Scalogne è in Sardagna 1884, 417.

scartà 'assottigliato' (detto del ferro): fero s. 3r19. Per alcuni riscontri cf. LEI, 13, 1354-5, che spiega tutte le forme a partire da carta 'carta di vetro' (cf. it. scartare 'scartavetrare'). Tale connessione, nel caso del nostro esempio e di altre attestazioni antiche (cf. infra), non sembra valida; si consideri d'altra parte che tra le fonti del LEl figurano anche repertori di area trentina e lombarda in cui non si fa alcuna menzione del ricorso alla carta abrasiva: cf. in particolare Aneggi, s.v. scartàr 'assottigliare il ferro rovente battendolo', e per il bresciano Melchiori, s.v. scartà 'battere il ferro' («Vale assottigliarlo quasi a guisa di carta. Locchè si fa per lo più col maglio»). Cf. ad ogni modo ferri scartati nella tariffa della muta del 1370 (Malossini 2003, 162); per (ferrum) scartatum in altri documenti medievali di area trentina cf. Varanini, Faes [2001] 2020, 1194 e 1196. schiete 'semplici, prive di ornamenti o aggiunte': sechie de legno s. $5 r 11$ (cf. invece sechie zente de fero 5r9). Cf. per es. Sella, s.v. scletus.

scudi 'armi da difesa' $5 r 5$.

sechie: s. zente de fero $5 r 9$; s. de legno schiete $5 r 11$. Il primo contesto può essere messo a confronto con $j$ secla zenta de fer 40 nell'Inventario giudicariese.

[segnaro]: part. pass.: segnà $8 \mathrm{v} 29$ (Ventura da Gar ${ }^{\circ}$ de' daro xvis libr. pro resto de coramo comprà da Avancin e s. ala soa raxon).

\section{sele 'selle' $5 r 4$.}

sevo 'sego' $5 r 15$.

smalzo 'strutto' o 'burro' $5 r 2$. Dall'alto-tedesco schmalz (REW, 7697; DEDI, s.v. smàlzo). Cf. ij chaze de smalz 42 nell'Inventario giudicariese; smalzo in Sardagna $1884,397$.

soma (unità di misura di peso): falze de zescauna s. [...] x s. veron. 3r21; panno [...] de zescauna s. [...] x s. veron. 4r11; zescauna s. de mercandaria $5 r 21$.

sonza 'sugna' $5 r 1$; vs once de s. $8 v 22$.

spala: s. lavorada $5 r 7$. Si tratta della pelle della spalla dell'animale. Cf. spala laborata nella tariffa della muta del 1370 (Malossini 2003, 162); spalla, -e anche in Sardagna 1884, 398 e 405.

[specificaro]: part. pass.: specificada $5 r 21$ (zescauna soma de mercandaria no s. indi suprascripti capitoli).

stopa 'stoppa' $5 r 13$.

stora 'stuoia' (come unità di misura per la vallonea): valania de zescauna s. [...] J libr. IIIJ s. 4v11. Cf. Sella, s.v. stora, dove si legge per l'appunto vendiderit valaniam ad storam (Verona, 1318); inoltre pro qualibet stara [ma sarà errore per stora] valanie nella tariffa della muta del 1370 (cf. Malossini 2003, 160).

tera 'terra': in $t$. de coloro 'polvere colorante, pigmento' 4v8. Da confrontare con terre a colori nella tariffa della muta del 1370 (cf. Malossini 2003, 162); si veda anche terra da color in Sardagna 1884, 402.

tine 'tini' (recipienti per olio): dove de lareso da t. da olio $3 r 10$. 
travi: legnamo distin[a] to ai t. e ale case de ogni nave - xx s. veron. 3v1; t. e canteri $4 \mathrm{v} 4$.

vache: carne de boy, de v. e de manzo 2v24; v. per exir de zescauna - J s. ver. $4 v 13$.

valanìa 'vallonea, sostanza colorante impiegata nella concia': $v$. de zescauna stora [...] J libr. IIIJ s. 4v11. Sul tipo cf. Bertoletti 2005, 513. La voce è presente nella tariffa della muta del 1370 (Malossini 2003, 160); si aggiunga valoniastampato con accento sulla $o$ - in Sardagna $1884,418$.

vedelo 'vitello': carne de v. 2v26; pelame de [...] v. 4r20.

[véndero]: part. pass.: vendù $1 r 7$ (Andrioto à v. - xxvIIJ $\div$ libr. de formay), 1 rg (à v. - xu libr. de formay), 8v19 (per coramo v. a Ventura da Gar ${ }^{\circ}$; vendue $1 r 10$ (xxv libr. de formay v. a Zohan de Cagnon), 1r11 (xLIIIJ libr. de formay v. al Conter).

veroneso 'veronese' (detto dell'olio): olio $v$. de zescaun mozo [...] xx dr. veron. 3v22; olio suprascripto v. e brexan e lorino $3 v 27$. verze: v. e erbe $4 v 17$.

veze 'botti': v. nove pro introitu de zescauna J s. ver. $4 v 15$. Dal latino tardo VEIA (REW, 9177; DEDI, s.v. véża). L'Inventario giudicariese attesta il derivato vezoy 27; cf. anche vèza, vezuòlo in Sardagna $1884,418$.

vino: v. de ogni fata de zescaun caro pro exitu - vIIJ s. ver. $4 v 10$.

zente 'cinte, cerchiate': sechie $z$. de fero $5 r 9$.

zentenaro 'unità di misura di peso': bronzo de zescaun z. [...] v s. veron. $2 v 1$. Cf. LEI, 13, 842 ss.; TLIO, s.v. centinaio (2).

zenture 'cinture' $5 r 19$. Si tratterà forse delle cinghie necessarie a fermare la sella (cf. TLIO, s.v. cintura, 4); nella tariffa della muta del 1370, in effetti, la menzione delle cinture segue immediatamente a quella delle selle (cf. Malossini 2003, 161). Si noti comunque che nei dialetti moderni del Trentino il tipo 'zentura' indica il collare di cuoio del bestiame (cf. Pedrotti 1936, 77; altra documentazione in LEl, 14, 274 ss.). 


\subsection{Antroponimi e toponimi}

Anche in questo paragrafo nel citare le forme del testo si rinuncia a segnalare lo scioglimento dei compendi. Come di consueto, si registrano gli antroponimi in due gruppi distinti (a seconda che la menzione sia diretta o indiretta).

\section{Antroponimi I}

Andrea $8 v 5$.

Andrioto $1 r 7$. A un magister Andriotus si fa riferimento nelle annotazioni in latino - di mano forse dell'autore delle registrazioni in volgare (vedi § 2) - presenti a c. $7 \mathrm{v}$.

Avancin 8v16,8v29. Alterato di 'Avanzo': cf. Clt, s.vv. Avancini e Avanzini, Avanzino.

Avancino 8v8 (A. e lachem), 8v12 (A. e lachem dela Val da Non). Cf. l'entrata precedente.

Conter 1r11. Ipocoristico di 'Buonincontro'. Cf. ad es. Boni(n)conter da Magnan B2v11 nel Memoriale di Graziadeo di Castel Campo; si veda anche Cesarini Sforza 1991, 66.

Franzolo 1r3. Da collegare al francese frange (cf. Olivieri 1923, 242) come il Fra(n)çono citato in Bertoletti 2005, 171.

Girardo da Cologna 1 r5.

lachem 8v8 (Avancino e I.), 8v12 (Avancino e I. dela Val da Non). Il tipo con e postonica, diffuso nel padovano ma estraneo al veronese (cf. Bertoletti 2005, 101 e nota 251), è attestato anche nel Memoriale di Graziadeo di Castel Campo, dove si menziona una certa Iachema B4r6; cf. inoltre lacheminus a Villa Banale nel 1391 (citato in Cesarini Sforza 1991, 159).

lachem del Ligo 8v24. Cf. l'entrata precedente.

Lafranco da Ledro, m. 1r1. Cf. Lafrancho in Bertoletti 2005, 529; Lafranchin in Stussi 1965, 275.

Martino cugnà de Martinel 1 r6.

Nicolò de Enselmo $8 \mathrm{v} 1$.

Peder de Nicolò da Brezo 8v30.

Ventura da Gar $^{\circ} 8 \mathrm{v} 20$, 8v28.

Zeschin 1r4. Da 'Francesco' (cf. Cesarini Sforza 1991, 51; Clt, s.v. Ceschìn, Ceschina, Ceschini).

Zohan de Cagnon 1 r10.

Zohan fradel de Avancin 8 v26.

\section{Antroponimi II}

Avancin, Zohan fradel de 8v26; Avancin, el fradel de 8v31.

Cagnon, Zohan de 1r10. Forse semplicemente 'grosso cane'; per riscontri di area trentina e per altre ipotesi etimologiche cf. Clt, s.v. Cagnóne, Cagnóni.

Enselmo, Nicolò de $8 \mathrm{v} 1$.

Ligo, lachem del 8v25. L'antroponimo si ripresenta anche altrove nella documentazione trentina medievale (cf. ad es. Cesarini Sforza 1991, 142). Ligo emerge in area toscana: cf. al proposito, in particolare, Brattö 1953, 130 e 145, dove il tipo è riportato a 'Federico' e la l- è giustificata ipotizzando una «pronuncia vezzeggiante o infantile»; per un riscontro in volgare si vedano i Testi pratesi di Serianni $(1977,643)$.

Martinel, Martino cugnà de 1 r6.

Nicolò da Brezo, Peder de 8v30. La lettura corretta, come si è segnalato in apparato nel $\S 2$, potrebbe essere Nicalò (ben diffuso a Verona: cf. Bertoletti 2005, 88). 


\section{Toponimi}

Alexandria: in sal grosa de A. o de Cipri $4 \mathrm{v} 21$.

Brezo 8v30. Corrisponde forse all'odierno Brez in Val di Non, di etimo discusso (cf. Mastrelli Anzilotti 2003, 330); da segnalare che a c. $7 v$, in una serie di annotazioni in latino forse attribuibili allo scrivente autore della tariffa (vedi § 2), si menziona un Hendricus de Brezio vallis Ananie.

Cipri: in sal grosa de Alexandria o de C. 4v21. Per le forme assunte dal toponimo nei testi antichi cf. Baglioni 2006, 272.

Cologna 1r5. In Trentino il toponimo (< COLŌNIA) si ritrova nel Tennese, ma anche in Val di Non e Valle del Chiese: cf. Mastrelli Anzilotti 2003, 135.

$\operatorname{Gar}^{\circ} 8 \mathrm{v} 20,8 \mathrm{v} 28$. Difficile identificare il toponimo, che potrebbe forse essere letto Gar(don)o (odierno Gardone [BS]) o Gar(dum)o, Gar(dun)o, «termine che un tempo indicava tutti i paesi della Val di Gresta» (Mastrelli Anzilotti 2003, 433).

Garzarìa 2v15. L'interpretazione di questa forma è molto incerta: il contesto (cori de G. e nostrani) fa pensare a un nome di luogo non locale, che potrebbe essere identificato con le 'Sgarzarie' veronesi, su cui cf. Faccioli 1965; il rapporto con questo toponimo urbano, tuttavia, dovrebbe essere chiarito. L'opposizione tra cuoio a Gazeria e nostrano si ripresenta nella tariffa del 1426 (cf. Reich 1903, 27); in quella del 1370 si legge pro qualibet caro [sic, forse per coro] gazarie (cf. Malossini 2003, 160).

Ledro 1r1. Il nome «dovrebbe derivare dall'etnico ALEUTRENSES per ALUTRENSES (citato da Plinio, Naturalis Historia III, 19)» (Mastrelli Anzilotti 2003, 124).

Val da Non 8v12. «Il nome Non potrebbe derivare dalla forma *Anaunis, de Anonis, de (A)nonis» (Mastrelli Anzilotti 2003, 323). 


\section{Bibliografia}

Aebischer, P. (1943). «La forme métathetique preta < petra en Italie». Zeitschrift für romanische Philologie, 63, 403-6.

AIS = Jaberg, K.; Jud, J. (Hrsgg) (1928-40). Sprach- und Sachatlas Italiens und der Südschweiz. Zofingen: Ringier.

ALD-I = Goebl, H. et al. (Hrsgg) (1988). Atlant linguistich dl ladin dolomitich y di dialec vejins I. 7 Bde. Wiesbaden: Reichert.

Aneggi = Aneggi, A. (1984). Dizionario cembrano (triangolo Sovér-MontesovérPiscine): parole e cose, frasi, modi di dire, proverbi del dialetto della Valle di Cembra. Revisione linguistica a cura di P. Rizzolatti. San Michele all'Adige: Museo degli Usi e Costumi della Gente Trentina.

Arcangeli, M. (1990). «Per una dislocazione tra l'antico veneto e l'antico lombardo (con uno sguardo alle aree contermini) di alcuni fenomeni fono-morfologici». L'ttalia dialettale, 53, 1-42.

Baglioni, D. (2006). La scripta italoromanza del regno di Cipro. Edizione e commenti di testi di scriventi ciprioti del Quattrocento. Roma: Aracne.

Battisti, C. (1906). La traduzione dialettale della "Catinia" di Sicco Polenton. Ricerca sull'antico trentino. Trento: Zippel. Estratto da Archivio Trentino, 19-21.

Battisti, C. (1910). «Lingua e dialetti nel Trentino». Pro Cultura, 1, 178-205.

Battisti, C.; Ventura, E. (1955). Atlante toponomastico della Venezia Tridentina: commento al foglio 14: Riva: I nomi locali del Basso Trentino Occidentale. Firenze: Il Rinascimento del Libro.

Benedetti, R.; Brugnolo, F. (2002). «Tra Lombardia e Veneto: uno zibaldone trentino del Quattrocento». Daniele, A. (a cura di), Antichi testi veneti. Padova: Esedra, 137-50.

Bertoletti, N. (2001). «Una lettera volgare del Trecento dal carcere di Modena». Studi linguistici italiani, 27, 233-47.

Bertoletti, N. (2005). Testi veronesi dell'età scaligera. Padova: Esedra.

Bertoletti, N. (2007). «Note in volgare veronese di Giacomo da Pastrengo (12741281 circa)». Lingua e Stile, 42, 13-71.

Bertoluzza, A. (a cura di) (1992). Atti del Il convegno sui dialetti del Trentino (1819-20 ottobre 1991). Trento: Centro culturale Fratelli Bronzetti.

Bocchi, A. (a cura di) (1991). Le lettere di Gilio de Amoruso, mercante marchigiano del primo Quattrocento. Edizione, commento linguistico e glossario. Tübingen: Max Niemeyer. https://doi.org/10.1515/9783110933789.

Bonelli, G.; Contini, G. (1935). «Antichi testi bresciani». L'Italia dialettale, 11, 115-51. Analisi linguistica (a cura di G. Contini) rist. in Contini 2007, 1199-212.

Bonfadini, G. (1983). «ll confine linguistico veneto-lombardo». Cortelazzo, M. (a cura di), Guida ai dialetti veneti. Padova: Cleup, 23-57.

Bonfadini, G. (1989). «La posizione linguistica della Val Rendena». Archivio per l'Alto Adige, 83, 1-74.

Bonfadini, G. (1992). «I dialetti trentini occidentali». Bertoluzza 1992, 35-60.

Bosshard, H. (1938). Saggio di un glossario dell'antico lombardo. Compilato su Statuti e altre Carte Medievali della Lombardia e della Svizzera Italiana. Firenze: Olschki.

Brattö, O. (1953). Studi di antroponimia fiorentina. Il Libro di Montaperti (An. mCCLX). Göteborg: Elanders.

Briquet, C.-M. (1923). Les Filigranes. Dictionnaire historique des marques du papier dès leur apparition vers 1282 jusqu'en 1600. 2ème éd. 4 vols. Leipzig: Hiersemann. 
Brusegan Flavel, E. (2005). «La Legenda di glorioxi apostoli misier sen Piero e misier sen Polo (codice Venezia, B.M.C. Correr 1497)». Quaderni veneti, 41, 7-108.

Cappelli, A. (2011). Lexicon abbreviaturarum. Dizionario di abbreviature latine ed italiane. 7 a ed. ampliata e rinnovata da M. Geymonat e F. Troncarelli. Milano: Hoepli.

Cesarini Sforza, L. (1991). Per la storia del Cognome nel Trentino. Nuova ed. con indici di G. Mastrelli Anzilotti. Firenze: Istituto di Studi per l'Alto Adige.

$\mathrm{Clt}=$ Caffarelli, E.; Marcato, G. (2008). I cognomi d'Italia. Dizionario storico ed etimologico. 2 voll. Torino: UTET.

Contini, G. (2007). Frammenti di filologia romanza. Scritti di ecdotica e linguistica (1932-1989). A cura di G. Breschi. 2 voll. Firenze: Edizioni del Galluzzo.

Cristelli, S. (in preparazione). Antichi testi trentini: (ri)edizione, commento linguistico e glossario [tesi di dottorato]. Zurigo: Università di Zurigo.

Curzel, E.; Gentilini, S.; Varanini, G.M. (a cura di) (2004). Le pergamene dell'Archivio della Prepositura di Trento (1154-1297). Bologna: il Mulino.

DEDI = Cortelazzo, M.; Marcato, C. (1992). Dizionario etimologico dei dialetti italiani. Torino: UTET.

DI = Schweickard, W. (2002-). Deonomasticon Italicum. Dizionario storico dei derivati da nomi geografici e da nomi di persona. 4 voll. più un suppl. Tübingen: Niemeyer.

Du Cange = Du Cange, C. Du F. (1883-87). Glossarium mediae et infimae latinitatis. Editio nova. Niort: Favre. Rist. anast. Graz: Akademische Druck- u. Verlagsanstalt, 1954.

DW = Grimm, J.; Grimm, W. (1984). Deutsches Wörterbuch von Jacob und Wilhelm Grimm. Hrsg. von der Deutschen Akademie der Wissenschaften zu Berlin in Zusammenarbeit mit der Akademie der Wissenschaften zu Göttingen. 33 Bde. München: Deutscher Taschenbuchverlag.

Faccioli, G. (1965). «Le "Sgarzarie"». Studi storici veronesi, 15, 177-86.

EWD = Etymologisches Wörterbuch des Deutschen (1989). Erarbeitet von einem Autorenkollektiv des Zentralinstituts für Sprachwissenschaft unter der Leitung von W. Pfeifer. 3 Bde. Berlin: Akademie-Verlag.

GDLI = Battaglia, S.; Bàrberi Squarotti, G. (a cura di) (1961-2002). Grande Dizionario della Lingua Italiana. 21 voll. (con 2 suppl. a cura di E. Sanguineti, 2004 e 2009). Torino: UTET.

Ghinassi, G. [1965] (2006). «Nuovi studi sul volgare mantovano di Vivaldo Belcalzer». Ghinassi, G., Dal Belcalzer al Castiglione. Studi sull'antico volgare di Mantova e sul "Cortegiano". Firenze: Olschki, 3-128.

Grazioli, M. (1987). «Riva veneziana. La finanza pubblica: le entrate ordinarie». Il Sommolago, 4, 49-102.

Höybye, P. (1974). «Glossari italiano-tedeschi del Quattrocento». Studi di filologia italiana, 32, 143-203.

LEI = Pfister, M.; Schweickard, W. (dal vol. 8, 2001); Prifti, E. (dal vol. 15(129), 2019) (a cura di) (1979-). Lessico etimologico italiano. Wiesbaden: Reichert.

Loporcaro, M. (2005-06) [ma 2007]. «I dialetti dell'Appennino tosco-emiliano e il destino delle atone finali nel(l'italo-)romanzo settentrionale». L'Italia dialettale, 66-7, 69-122.

Loporcaro, M. (2018). Gender from Latin to Romance: History, Geography, Typology. Oxford: Oxford University Press. https://doi.org/10.1093/ oso/9780199656547.001.0001. 
Magagna, F. (1995). «Una testimonianza di volgare scritto in una famiglia quattrocentesca trentina: il "Memoriale" di Graziadeo di Castel Campo». Banfi, E. et al. (a cura di), Italia settentrionale: crocevia di idiomi romanzi = Atti del convegno internazionale di studi (Trento, 21-23 ottobre 1993). Tübingen: Niemeyer, 289-98. https://doi.org/10.1515/9783110910346.289.

Malossini, A. (2003). «lstituzioni e società a Riva dagli inizi del Trecento alla dominazione Veneziana». Il Sommolago, 20(1), 5-222.

Martinelli, F. (2000). «ll dazio e la Muda di Riva in epoca veneziana». /l Sommolago, 17(1), 5-24.

Mastrelli Anzilotti, G. (1992). «I dialetti trentini centrali». In Bertoluzza (1992, 7-20).

Mastrelli Anzilotti, G. (2003). Toponomastica trentina: i nomi delle località abitate. Trento: Provincia autonoma di Trento Servizio beni librari e archivistici.

Melchiori = Melchiori, G.-B. (1817). Vocabolario bresciano-italiano. Brescia: Franzoni e Socio.

Mussafia, A. (1873). Beitrag zur Kunde der norditalienischen Mundarten im XV. Jahrhunderte. Wien: s.n.

Olivieri, D. (1923). I cognomi della Venezia Euganea. Genève: Olschki.

Pedrotti, G. (1936). Vocabolarietto degli arnesi rurali della Val d'Adige e delle altre valli trentine. Trento: Società per gli Studi Trentini.

Pedrotti, G.; Bertoldi, V. (1930). Nomi dialettali delle piante indigene del Trentino e della Ladinia Dolomitica presi in esame dal punto di vista della botanica della linguistica e del folclore. Trento: G.B. Monauni.

Pellegrini, G.B. (1976). «Le denominazioni di alcune conifere nei dialetti friulani». Scritti in onore di Giuliano Bonfante, vol. 2. Brescia: Paideia, 605-38.

Pellegrini, G.B. (1977). Carta dei dialetti d'Italia. Pisa: Pacini.

Piccard, G. (1987). Wasserzeichen Hirsch. Stuttgart: Kohlhammer.

Plomteux = Plomteux, H. (1975). I dialetti della Liguria orientale odierna: la Val Graveglia. 2 voll. Bologna: Pàtron.

Postinger, C.T. (1901). «Documenti in volgare trentino della fine del Trecento relativi alla cronaca delle Giudicarie: lotte fra gli Arco, i Lodron, i Campo ed il Vescovo di Trento». Atti dell'Accademia roveretana degli Agiati, serie 3, 7(1/2), 21-235.

Quaresima = Quaresima, E. (1964). Vocabolario anaunico e solandro, raffrontato col trentino. Venezia; Roma: Istituto per la collaborazione culturale.

Reich, D. (1903). «L'antico dazio di Riva». Tridentum, 1, 25-8.

Ressegotti, D. (2010-11). Gli Statuti della Confraternita dei Battuti di Trento: nuova edizione e commento linguistico [tesi di laurea magistrale]. Trento: Università degli Studi di Trento.

Ressegotti, D. (2012). «"Spala de portadoro": una nota quattrocentesca in volgare trentino». Studi Trentini. Storia, 91(1), 191-202.

REW = Meyer-Lübke, W. (1935). Romanisches Etymologisches Wörterbuch. 3. Ausg. Heidelberg: Winter.

Ricci = Ricci, V. (1904). Vocabolario trentino-italiano. Trento: Zippel.

Rigobello = Rigobello, G. (1998). Lessico dei dialetti del territorio veronese. Verona: Accademia di Agricoltura Scienze e Lettere di Verona.

Rohlfs = Rohlfs, G. (1966-69). Grammatica storica dell'italiano e dei suoi dialetti. 3 voll. Torino: Einaudi.

Rosalio, M.R. (1979). Studi sul dialetto trentino di Štivor (Bosnia). Firenze: La Nuova Italia Editrice. 
Salvadori = Salvadori, G.B. (1999). Vocabolario del dialetto di Roncone. Roncone: Rendena.

Salvioni, C. (1914). «Centuria di note etimologiche e lessicali». Romania, 43, 371402,560-86. https://doi.org/10.3406/roma.1914.4803; https://doi. org/10.3406/roma.1914.4815. Rist. in Salvioni 2008, 4: 1075-133, dove si riproduce la paginazione originale (cui ci si riferisce nel presente saggio).

Salvioni, C. (1916). «Dell'elemento germanico nella lingua italiana; A proposito di un libro recente». Rendiconti del Reale Istituto Lombardo di scienze e lettere, 49, 1011-67. Rist. in Salvioni 2008, 4: 1134-91, dove si riproduce la paginazione originale (cui ci si riferisce nel presente saggio).

Salvioni, C. (2008). Scritti linguistici. A cura di M. Loporcaro et al. 5 voll. Bellinzona: Edizioni dello Stato del Cantone Ticino.

Sardagna, G. (1884). «Documenti circa il feudo della Muta in Riva di Trento». Archivio veneto, serie 2, 28(1/2), 11-35.

Schatz = Schatz, J. (1955). Wörterbuch der Tiroler Mundarten. Für den Druck vorbereitet von K. Finsterwalder. Innsbruck: Universitätsverlag Wagner.

Schmeller $=$ Schmeller, J.A. (1827-36). Bayerisches Wörterbuch. 3 Bde. Stuttgart: Cotta.

Schneller, C. (1881). «Statuten einer Geißler-Bruderschaft in Trient aus dem XIV. Jahrhundert». Zeitschrift des Ferdinandaeums für Tirol und Vorarlberg, 25, 3-54.

Schneller, C. (1898). Tridentinische Urbare aus dem dreizehnten Jahrhundert. Mit einer Urkunde aus Judicarien von 1244-1247. Innsbruck: Wagner.

Sella = Sella, P. (1944). Glossario latino italiano. Stato della Chiesa - Veneto Abruzzi. Città del Vaticano: Biblioteca Apostolica Vaticana.

SellaE = Sella, P. (1937). Glossario latino emiliano. Città del Vaticano: Biblioteca Apostolica Vaticana.

Serianni, L. (1977). Testi pratesi della fine del Dugento e dei primi del Trecento. Firenze: Accademia della Crusca.

Stenico, R. (1979). «Dazio al Passo del Tonale, 6 agosto 1460-13 ottobre 1461». Studi trentini di scienze storiche, 80, 15-77.

Stenico, M. (2013). «Una carta rivana del primo Trecento in volgare». Studi trentini. Storia, 92, 157-72.

Stussi, A. (1965). Testi veneziani del Duecento e dei primi del Trecento. Pisa: Nistri-Lischi.

Tagliani, R.; Bino, C. (2011). «Testi confraternali e "memoria” della Passione a Brescia fra Tre e Quattrocento. Il Planctus virginis Mariae e la Sententia finalis iudicii dei Disciplini di San Cristoforo». Filologia \& Critica, 36, 75-126.

Tiraboschi = Tiraboschi, A. (1873). Vocabolario dei dialetti bergamaschi antichi e moderni. 2a ed. Bergamo: Bolis.

$\mathrm{TLIO}=$ Tesoro della Lingua Italiana delle Origini. http://tlio.ovi.cnr.it/.

Tomasin, L. (2004). Testi padovani del Trecento. Edizione e commento linguistico. Padova: Esedra.

Tomasini, R. (1990). Il dialetto della Val Rendena. San Michele all'Adige: Museo degli Usi e Costumi della Gente Trentina.

Tomasini, G. (1955). Le palatali nei dialetti del Trentino: appunti sopra un'indagine linguistica. Roma: Bocca Editori.

Tuttle, E.F. (1991). «Considerazione pluristratica sociale degli esiti di AU e AL + alveodentale nell'Italia settentrionale». Kremer, D. (éd.), Actes du XVIIIe Congrès International de Linguistique et de Philologie Romanes (Université de Trèves [Trier], 1986), vol. 3. Tübingen: Niemeyer, 571-83. 
Varanini, G.M. [1996] (2020). «Itinerari commerciali secondari nel Trentino bassomedioevale». Varanini 2020, 2: 1109-39.

Varanini, G.M. [2004] (2020). «L'economia. Aspetti e problemi (XIII-XV secolo)». Varanini 2020, 2: 1041-108.

Varanini, G.M. [2011] (2020). «Note sulla documentazione fiscale di Riva del Garda nel Quattrocento». Varanini 2020, 2: 1151-74.

Varanini, G.M. (2020). Studi di storia trentina. A cura di E. Curzel e S. Malfatti. 2 voll. Trento: Università degli Studi di Trento, Dipartimento di Lettere e Filosofia.

Varanini, G.M.; Faes, A. [2001] (2020). «Note e documenti sulla produzione e sul commercio del ferro nelle Valli di Sole e di Non (Trentino) nel Trecento e nel Quattrocento». Varanini 2020, 2: 1175-213.

Zingerle, W. (1900). «Eine wälschtirolische Handschrift». Zeitschrift für romanische Philologie, 24, 388-94. https://doi.org/10.1515/ zrph.1900.24.1-4.388. 


\title{
La biblioteca di una filantropa Libri e letture di Stéphanie Omboni (1839-1917)
}

Vincenzo Vozza

Ricercatore indipendente

\begin{abstract}
The aim of this article is to deepen the complex personality of Stéphanie Etzerodt Omboni (1839-1917), the Anglo-Belgian philanthropist and activist for women's rights who lived in Padua in the second half of the nineteenth century by considering which readings were at the basis of her cultural, human, and spiritual formation, with a special account on the books or authors marked her philanthropic work or nourished her intellect or her curiosity, which kind of literary genres could be found in her library, in which languages, their quality, and their extent. The sources for this research are the correspondence between Stéphanie Omboni and some of the most preeminent personalities of her time (particularly with the anthropologist Paolo Mantegazza, the feminist and writer Sibilla Aleramo and the philosopher Gaetano Meale) and the remains of her family library, a partial book collection that constituted the much larger personal library of the Omboni couple, currently kept at the Museum of the Education of the University of Padua.
\end{abstract}

Keywords Stéphanie Omboni. Middle-class library. First feminism. Cultural History.

Sommario 1 Introduzione. - 2 Per la fisionomia di una lettrice di fine Ottocento: la biblioteca domestica di Stéphanie Omboni. - 3 Libri, letture e brogliacci. Ciò che emerge dagli scambi epistolari. - 3.1 Paolo Mantegazza. - 3.2 Rina Faccio/Sibilla Aleramo. 3.3 Gaetano Meale, alias Umano. - 4 Conclusioni.

$\begin{array}{lll} & \text { Peer review } & \\ \text { Edizioni } & \text { Submitted } & \text { 2021-06-01 } \\ \text { Ca'Foscari } & \text { Published } & 2021-08-25 \\ & \text { 2022-02-17 } \\ & \text { Open access } & \end{array}$

(c) 2022 | () Creative Commons Attribution 4.0 International Public License 


\section{Introduzione}

La recente pubblicazione della biografia di Stéphanie Etzerodt Omboni (1839-1917) a cura di Stefania Masiero (2020) ha ridato vigore agli studi sulla personalità complessa e inesauribile della filantropa e benefattrice anglo-belga, che nell'arco dei cinquant'anni trascorsi a Padova ha lasciato un segno indelebile del suo passaggio nelle opere sociali da lei avviate. Il volume di Masiero offre altresì una ricca appendice, costituita soprattutto da lettere, articoli di giornale e fotografie d'epoca. Questi documenti, interrogati di volta in volta secondo prospettive differenti, aprono nuove piste che ampliano significativamente l'orizzonte della ricerca. Limitandosi alla sezione epistolare, i temi centrali degli scambi sono le attività a sostegno della causa emancipazionista e le opere di beneficenza in favore della società padovana, ma non mancano riferimenti occasionali ai fatti di cronaca, alle piccole o grandi incombenze quotidiane, e, non ultime, alle novità editoriali, alle letture, agli acquisti o ai prestiti librari.

Data la prossimità dei coniugi Omboni all'élite culturale internazionale e padovana - Giovanni, infatti, era professore di geologia presso la Regia Università (Canadelli 2015) - non era insolito trovare tra le righe della corrispondenza di Stéphanie Etzedodt il riferimento a qualche lettura edificante, ad un autore o ad una autrice contemporanei, oppure, nel caso di interlocutori più intimi alla donna, il richiamo alle bozze di un'opera letteraria in corso di pubblicazione sulla quale veniva chiamata ad esprimersi.

In un contesto vivace come quello emancipazionista, che incitava un nuovo protagonismo femminile, la costruzione delle relazioni intellettuali, vere e proprie alleanze muliebri, si rinsaldava sulla condivisione di interessi, di un orizzonte ideale di valori che si traducevano in azioni concrete, e poteva accadere che i rapporti più longevi nascessero e si rinsaldassero, benché a distanza, via lettera. E forse grazie alla voluminosa produzione epistolare dell'Otto-Novecento che si è potuto sottrarre all'oblio della storiografia contemporanea la straordinarietà dell'ordinario. La febbrile necessità delle donne di raccontarsi e raccontare il mondo che le circonda (al principio del lungo processo di autorappresentazione) si manifesta in un genere letterario, quello epistolare, che rompe alcuni cliché proprî del paradigma narrativo maschile. Queste nuove prospettive sulla quotidianità hanno permesso così di restituire alcune informazioni apparentemente marginali rispetto alle esigenze narrative della grande Storia, che diventano tuttavia sostanziali quando ci si pone come obiettivo la ricerca delle fonti per la storia culturale.

Scopo di questo saggio è di restituire complessità alla fisionomia di Stéphanie Etzerodt Omboni considerando quali letture siano state alle base della sua formazione culturale, umana e spirituale, di quali libri o di quali autori abbia conservato memoria o ne abbiano se- 
gnato l'opera filantropica, di cosa abbia nutrito l'intelletto per curiosità o per circostanza, di quali generi letterari e in quale lingua, in che misura e in che quantità. Le fonti per questa ricerca sono principalmente le lettere che costituiscono il suo epistolario, parziale ma sufficientemente ampio da dare un risultato quantomeno indiziario. ${ }^{1}$ A questo si accosterà la ricognizione del piccolo fondo librario che costituiva la ben più ampia biblioteca personale dei coniugi Omboni, attualmente conservato presso il Museo dell'Educazione dell'Università di Padova. ${ }^{2}$

\section{Per la fisionomia di una lettrice di fine Ottocento: la biblioteca domestica di Stéphanie Omboni}

Immagine della tipica famiglia intellettuale borghese dell'Italia postunitaria, gli Omboni potevano accedere facilmente al fiorente mercato librario italiano e internazionale, favorito dalla legislazione sabauda, dalla circolazione degli almanacchi bibliografici e dagli investimenti d'impresa nell'arte tipografica. ${ }^{3}$ In una lettera del 1903 all'amica Rina Faccio (poi nota con lo pseudonimo di Sibilla Aleramo), Stéphanie Omboni confessa che la biblioteca di famiglia è così carica di libri da ammassarsi gli uni contro gli altri sugli scaffali, e che le riviste alle quali lei e il marito sono abbonati sono talmente tante che molto spesso rimangono intonse, "sans être même feuilletés». ${ }^{4}$ Se si osserva invece oggi la consistenza della biblioteca della Ombo-

1 Oltre alle lettere pubblicate in Masiero 2020, 205-448, si segnala anche la corrispondenza della Omboni con Sibilla Aleramo pubblicato in Vozza 2020.

2 Al momento della dismissione della sede dell'Istituto per l'infanzia abbandonata, sito in Via Campagnola (Padova), vennero prelevate numerose suppellettili appartenute alla residenza degli Omboni e divenute poi parte del patrimonio dell'istituto in virtù del lascito testamentario di Stéphanie Etzerodt (Vozza 2017). Tra queste, oltre al mobilio, andarono a costituire il Museo dell'Educazione anche i libri della coppia.

3 Nella seconda metà dell'Ottocento, anche grazie alla Legge Casati (1859) sull'istruzione obbligatoria, si assistette ad un incremento della stampa e della circolazione di libri e periodici: si vedano, a tal proposito, le statistiche sulla produzione libraria nel Sommario delle statistiche $(1968,46)$. Nel 1861, anno dell'Unità, le pubblicazioni riportate nel Catalogo dei libri italiani dell'Ottocento sono 4.802. Le opere stampate in Italia passarono da 3.314 nel 1836 a 4.243 nel 1863, fino a 6.317 nel 1872 (Ragone 1999, 16); il numero di periodici, compresi i quotidiani, passò da 220 nel 1845 a 450 nel 1864, a 1.126 nel 1872 (Alaimo 1991, 30). Si veda a tal proposito anche Vittoria 2011; Finocchi, Gigli Marchetti 2000; Chemello 2002.

4 «Vous oubliez que je suis une illettrée et que je n'ai jamais le temps d'ouvrir un livre, à peine le temps de jeter un coup d'œil sur le journal du jour. Une immense quantité d'imprimés hebdomadaires ou mensuels, auxquels il a fallu s'abonner, arrivent chez nous et preument rang sur les rayons de la bibliothèque sans être même feuilletés. Mon mari lit le N.A. [= Nuova Antologia] à son cabinet de lecture, et je trouverai moyen de l'avoir pour y lire le roman de son illustre directeur» (Lettera di Stéphanie Omboni a Rina Faccio del 1903, 18 agosto è pubblicata in Vozza 2020, 110-12). 
ni, conservata in un armadio espositivo del Museo dell'Educazione di Padova, la sensazione di straniamento è grande: di quella biblioteca domestica non risultano che pochi titoli, tali da non rappresentare adeguatamente la complessa fisionomia della filantropa, né tantomeno del marito.

La dispersione dei volumi che dovevano affollare la casa degli Omboni in Via del Torresino 3, a Padova, fu tuttavia una conseguenza diretta delle ultime volontà di Stéphanie. Nel testamento, infatti, redatto un anno prima della sua morte, la vedova Omboni nomina suo erede universale l'Istituto per l'infanzia abbandonata, l'opera per la quale si era spesa con zelo, e incarica il marchese di Pietro Buzzacarini, l'esecutore testamentario, di alienare i beni non ancora assegnati secondo il proprio giudizio. ${ }^{5}$ Tra questi, vi sono anche i libri e i documenti della famiglia. Fatta eccezione per i libri, gli appunti e la collezione di minerali di Giovanni Omboni, che andarono ad arricchire il fondo della facoltà di Geologia della Regia Università di Padova, la biblioteca domestica venne verisimilmente dispersa per lo più nei due anni successivi alla morte di Stéphanie (1917-1919), per intervento dello stesso Buzzacarini ma anche dei colleghi e amici degli Omboni, tra i quali l'assistente e successore di Giovanni alla cattedra di geologia Giorgio Dal Piaz (1872-1962), il mineralista Angelo Bianchi (1892-1970) e il medico oculista Giuseppe Albertotti (18511936). ${ }^{6}$ Il mobilio della villa, insieme ad altre suppellettili e ai libri, andò a costituire l'arredamento della direzione dell'Istituto fino alla sua soppressione (fine anni Novanta) e alla collocazione degli stessi nei locali del Museo dell'Educazione (Vozza 2017, 141).

Ad oggi manca un inventario che assegni un record catalografico ai libri della Omboni; pertanto, si sono riportati i titoli così come compaiono a scaffale (si veda l'Appendice di questo saggio). È possibile riconoscere i libri appartenuti a Stéphanie Omboni dall'ex libris stampigliato con inchiostro blu sul frontespizio, o recanti la firma autografa della filantropa. Si tratta di trenta pezzi, alcuni dei quali appartenenti a serie numerate in collana (come l'opera omnia del poeta Friedrich Schiller, della quale restano quattro dei dodici volumi di cui era composta) corrispondenti a ventisei opere, non solo letterarie.

5 Si veda il testamento di Stéphanie Omboni pubblicato in Masiero 2020, 457-62.

6 Una prova documentaria di questi interventi si legge in una nota manoscritta di Giuseppe Albertotti sul recto di una busta che contiene le lettere di Paolo Mantegazza rivenute tra gli effetti di Stéphanie Omboni: «Padova 14 marzo 1919. In casa Omboni col prof. Dal Piaz il direttore Bianchi mi regala le lettere accluse del Mantegazza alla signora Omboni deplorando che sieno andate smarrite quelle del Mantegazza al prof. Omboni» (di mano di Giuseppe Albertotti). La busta è a sua volta contenuta in una busta intestata R. Biblioteca Universitaria di Padova, su cui è annotato: «Lettere di Paolo Mantegazza a Stéphanie Omboni. Dono del prof G. Albertotti a mezzo prof. Faggiotto», deposito avvenuto nel 1933. Si veda Padova, Biblioteca Universitaria (BU), Fondo manoscritti, ms. provv. 217. 
Una prima e consistente parte dello scaffale è costituita da ben otto volumi inerenti allo studio delle lingue, sussidi per l'apprendimento della grammatica e della sintassi (inglese, francese e tedesca) nonché dizionari bilingui. Alcuni di questi, come il vocabolario inglese-tedesco curato dal lessicografo e linguista Jacob Heinrich Kaltschmidt (1849), potrebbero costituire quel bagaglio librario che seguì Stéphanie negli anni della sua formazione, dal Belgio alla Russia fino a Milano. Anche dopo il matrimonio e il trasferimento a Padova Stéphanie continuò a viaggiare, sia al seguito del marito durante le spedizioni sulle Alpi, sia per conto proprio, raggiungendo Alessandrina Ravizza a Milano e ai bagni termali in Svizzera, o Sibilla Aleramo nell'Agro pontino o ancora la sorella Margarethe a Millstat, in Carinzia. Non stupisce dunque che un secondo consistente nucleo sia costituito da opere di geografia, come i primi due volumi dei Précis de géographie universelle (1829) di Conrad Malte-Brun, ma anche una guida del Lazio curata dal Touring Club italiano e un catalogo pubblicitario di alberghi e itinerari della penisola pubblicato dall'editore Hachette (1888).

Un terzo nucleo di libri è quello riguardante l'attività filantropica della Omboni e la sua crociata per la moralità pubblica. Si trova, ad esempio, la Nuova legge sulle opere pie del 1891, un codice legislativo ampiamente sottolineato e commentato a margine con noterelle autografe a matita. Questo testo, di natura giuridica, venne utilizzato dalla Omboni per la stesura degli statuti e dei regolamenti delle associazioni caritatevoli da lei ispirate. Tra le pubblicazioni che corroborano la fisionomia di educatrice e moralizzatrice della condizione femminile si trova l'Ecole de la Pureté di Emma Reichenbach Pieczynska. Si tratta di un saggio sull'educazione sessuale destinato alle giovani madri di famiglia, pubblicato per la prima volta nel 1898 (Stéphanie Omboni possiede la quarta edizione del 1902), un libro molto lodato da alcuni moralisti ma, sotto certi aspetti, controverso per l'uso di categorie religiose e scientiste per introdurre le donne alla vita sessuale. L'opera costitutiva la tesi che la Reichenbach avrebbe dovuto discutere all'Università di Ginevra per conseguire il dottorato, ma le sue condizioni di salute (una sordità invalidante) la ostacolarono nel raggiungimento dell'obiettivo (Käppeli 1990, 79102). ${ }^{7}$ Si trovano, ancora, alcune novelle morali e satiriche scritte

7 Emma Reichenbach (1854-1927), persa l'opportunità di conseguire il dottorato, conobbe Josephine Butler, la fondatrice della International Abolitionist Federation, un comitato internazionale che si adoperava per porre fine alla prostituzione, una crociata nella quale si impegnò anche Stéphanie Omboni con la Lega contro la tratta delle bianche. Nel 1891 fondò l'Union des femmes de Genève e nel 1900 il Bund Schweizerischer Frauenvereine. Nel 1906 contribuì alla fondazione della Lega svizzera dei consumatori e nel 1915 partecipò alla Commissione nazionale per l'educazione. Nell'Ecole de la pureté descrive la prospettiva di educazione sessuale cristiana per le giovani don- 
da Louis Reybaud (Jerome Paturot à la recherche d'une position sociale, 1847), da Louis Desnoyers (Les Avventures de Jean-Paul Choppart, 1836) e dalla scrittrice e filantropa inglese Maria Edgeworth (1857). Due numeri di periodici concludono questa rassegna: L'Ami des Enfants, serie di fascicoli sull'educazione infantile pubblicati a Parigi dall'abate Roussel, e The Englishwoman's Yearbook del 1887, un annuario curato dalla riformatrice sociale inglese Louisa Maria Hubbard e destinato alle donne, contenente un elenco delle diverse esperienze filantropiche aggiornate ad annum. Tra i romanzi di critica sociale, poi, si trova anche il primo dei due volumi di Sans famille (1886) di Hector Malot. Come Honoré de Balzac per Parigi o Charles Dickens per Londra, anche Malot voleva rappresentare la società contemporanea e le sue contraddizioni: il centro e le periferie, l'abisso tra le diverse classi sociali e più in particolare tra la borghesia e il nascente proletariato urbano, ma anche la povertà e l'emarginazione dei fanciulli nella società industrializzata, tutti temi cari a Stéphanie Omboni e fondanti la sua attività filantropica a favore del suo Istituto per l'infanzia abbandonata in Padova.

Un quarto nucleo, ancora, è costituito da alcuni saggi storico-letterari, come quello su Albertino Mussato (1884) scritto da Antonio Zardo e la Vita di San Francesco di Sales compilata sui manoscritti originali da Andrè-Jean-Marie Hamon (1885, opera della quale resta soltanto il secondo volume). Non si possono non menzionare le copie di volumi con dedica, Il Gabinetto di geologia della R. Università di Padova con una nota autografa di Giovanni Omboni alla moglie ( Alla mia diletta compagna, questa breve storia di quasi 30 anni di vita padovana», 1898), e la pubblicazione dello studioso e 'collezionista' Julius Pée, con nota autografa dell'autore («Aan Mevrouw Omboni, van den verzamelaar», datata Bonn, 4 aprile 1895) con le lettere di personali di Tine, la sfortunata moglie del poeta Edouard Douwes Dekker. La pubblicazione della corrispondenza di Everdina Huberta (Tine) van Wijnbergen, venne vissuta da Stéphanie Omboni con un certo fastidio, poiché non desiderava diventare oggetto vivente del pettegolezzo suscitato dalla critica letteraria belga. La van Wijnber-

ne: «Et ailleurs? Que faites-vous dans la philanthropie, dans toutes les œuvres de charité, sinon porter aux malheureux un cœur et des conseils maternels? Quel est l'idéal de la diaconesse, de la sœur de charité ? N'est-ce pas cette union de patience et de courage - le courage de faire souffrir s'il le faut - qui caractérise le mieux un cœur de mère?» (Reichenbach Pieczynska 1902, IX) e ancora «c'est une école qu'il faut à la pureté, - une école, dans toute l'acception du terme. Son programme doit comprendre des informations positives, des notions précises sur le corps humain et ses lois, mais aussi, la recherche de principes moraux de conduite et la science de leur application. Enfin, il en est de la pureté comme de tout autre objet d'enseignement; pour en prendre une connaissance intime, il faut encore l'exercice personnel, qui seul fournit les données indispensables de l'expérience. C'est à celui qui fait la volonté de Dieu qu'est promise la connaissance de sa pensée» (Reichenbach Pieczynska 1902, XIX). 
gen, infatti, era stata una grande amica della famiglia Etzerodt, e malgrado la differenza di età con Stéphanie, coltivò con la giovane un rapporto profondo, quasi materno, di amicizia e confidenza. Tine visse una vita difficile a causa delle avventatezze e delle infedeltà del marito, motivo per cui decise di trasferirsi in Italia non senza l'aiuto economico di Stéphanie e Giovanni Omboni. Dopo la morte della van Wijnbergen, avvenuta nel 1874 a Venezia, Julius Pée scrisse più volte e con insistenza alla Omboni per avere informazioni dettagliate sugli ultimi anni della donna: Stéphanie accettò soltanto per restituire onore e verità sulla vita dell'amica (Masiero 2020, 50-1). ${ }^{8}$

Un altro libro che vale la pena segnalare è quello di Annie Besant, Esoteric Christianity, pubblicato nel 1902 per la Theosophical Society di Londra. L'interesse della Omboni per la ricerca di un'armonia tra lo spirito e la natura è comune a molte delle emancipazioniste che avevano le proprie radici culturali nell'utopismo umanitarista e nell'aspirazione di creare una società nuova e più moralizzata. Le donne che si avvicinarono alla Società Teosofica, alla quale Stéphanie Omboni non aderirà direttamente ma che osserverà con interesse, erano influenzate dal riformismo progressista, dalle teorie evoluzioniste che avevano trovato altri spazi al di fuori della religione cristiana. Inoltre, attraverso la pratica dello spiritismo, ovvero la possibilità di trovare prove concrete e scientifiche dell'esistenza dell'aldilà, vedeva nella via esoterica della conoscenza la risposta alle domande fondamentali sulla vita umana in un percorso al tempo stesso spirituale e scientifico (Masiero 2020, 64-6). È tuttavia complesso stendere un profilo della religiosità di Stéphanie Omboni, una donna tanto spirituale quanto figlia del laicismo anticlericale proprio delle posizioni più liberali del tardo Ottocento. Non deve sorprendere, dunque, veder coesistere nella prassi filantropica della benefattrice l'esaltazione delle virtù morali che potevano essere rintracciate e condivise anche dal cristianesimo liberale, da Lamennais a Gioberti, pur rivendicando l'autonomia e l'indipendenza del percorso emancipazionista dalla Chiesa cattolica. Ecco dunque manifestarsi nella Omboni, fin dalla giovinezza, l'interesse per la lettura delle ricerche sul 'Gesù storico', dapprima nelle pagine de La Vie de Jésus (1863) di Ernst Renan e poi nel Das Leben Jesu kritisch bearbeitet (1835) del filosofo hegeliano David Strauss. Nel 1876, ormai giunta a Padova, Stéphanie Omboni si cimenterà nella traduzione di un'altra importante opera di

8 Nella lettera del 9 settembre 1894, in risposta a Julius Pée (Masiero 2020, 431) Stéphanie Omboni scrive: «Monsieur, non posso dirvi quanto la vostra lettera mi abbia commosso e compiaciuto suscitando in me i ricordi più dolci e cari di un'amica che ho adorato e della quale coltivo il ricordo per quella sua gentilezza ed eccellenza che mai più ho incontrato in tutta la mia vita mia. Penso di poter dire che è stata l'unica persona che ho incontrato che non mi ha mai causato la minima delusione. Come l'ho conosciuta fin dall'inizio, così è rimasta per quindici anni nell'intimità più perfetta, nell'amicizia più tenera». 
Strauss, Der alte und der neue Glaube (1872), pubblicandola col titolo italiano L'antica e la nuova fede..$^{9}$ Nel saggio, Strauss riconduceva i contenuti delle Scritture alle strutture del discorso mitologico, riconoscendo l'esistenza di un patrimonio di immagini e concetti metafisici comuni a tutta l'umanità. Questo approccio universalista si ritroverà assai di frequente nel pensiero e nelle opere filantropiche della Omboni così come di altre benefattrici italiane del XIX secolo.

Tra i libri della Omboni si trova anche il romanzo satirico e anticlericale The Soul of a Priest, pubblicato dal duca Pompeo Litta Visconti Arese per un editore londinese nel 1907. È la storia di Renato, un giovane intelligente ma ingenuo (ricorda, nella sua fisionomia, il Candido di Voltaire), che decide di diventare sacerdote per fuggire la corruzione del mondo, ma, deluso anche dalla corruzione della Chiesa, si ritrova a meditare in un limbo esistenziale sulla propria coscienza e sulla verità. La storia raccontata dal duca Litta trova ispirazione dalle vicende della propria famiglia, fedele alla causa rivoluzionaria francese e parte integrante dell'amministrazione italiana nel progetto napoleonico per la Repubblica Cisalpina. Uno zio dell'autore, Giulio Renato, che fu cavaliere dell'Ordine di Malta naturalizzato russo, sembra ispirare la figura del protagonista, mentre l'altro zio, il cardinale Lorenzo, fu uno dei più fedeli principi della Chiesa durante l'esilio di Pio VI, tale da ispirare un altro personaggio del romanzo del nipote Pompeo.

\section{Libri, letture e brogliacci. Ciò che emerge dagli scambi epistolari}

Come si è visto nel paragrafo precedente, ciò che resta della libreria di Stéphanie Omboni è un numero esiguo di pezzi, sufficiente per poter avere un quadro generale ma certamente non esaustivo. A questa lacuna materiale suppliscono in parte le informazioni che si possono ricavare dalla ricca corrispondenza di Stéphanie Omboni con alcune delle personalità più note della cultura e della scienza a lei contemporanee, con l'élite intellettuale italiana ma anche internazionale.

A riprova dell'ampiezza degli interessi e delle relazioni di Stéphanie Omboni si possono portare alcuni esempi tratti dalla documentazione fino ad oggi raccolta. Dallo scambio con di Tine si viene a sape-

9 Così scrive Tine il 26 novembre 1863 (Masiero 2020, 251) in una lettera dalla quale si viene a sapere che Stéphanie Omboni avrebbe suggerito all'amica la lettura della Vita di Cristo di Renan: «Concordo con te sul fatto che l'essenza del Vangelo è l'amore e che amare come Cristo ci prescrive è sublime. Non ho letto la Vita di Cristo di Renan. Proverò a ottenerlo, ma in questo momento non ho requie, ho troppe preoccupazioni». Si veda anche la traduzione di Strauss (1876). Per un approfondimento si veda anche Gazzetta 2013. 
re che la giovane Omboni aveva ricevuto una copia dei due romanzi di Edouard Douwes Dekker, il Max Havelaar of de koffij-veilingen der Nederlandsche Handel-Maatschappy (Max Havelaar, ovvero Le aste del caffè della Società di Commercio olandese) pubblicato nel 1860 (Masiero 2020, 233) e Ideën, pubblicato nel 1862 (Masiero 2020, 251-5). ${ }^{10}$ In particolare, nel Max Havelaar Douwes Dekker si era lanciato in un'aspra critica antimperialista, basata sulle vicende di cui era stato protagonista durante la permanenza a Giava, tale da scuotere l'opinione pubblica olandese ed incidere profondamente sulla percezione che i cittadini e la politica avevano dello sfruttamento nelle colonie. È lo stesso autore che, sul finire della terza delle quattro parti in cui è suddiviso il libro, dichiara che il suo romanzo si pone verso i diritti violati dei coloni indocinesi come il libro di Harriet Beecher Stowe, Uncle Tom's Cabin (1852), si poneva nei confronti dei diritti degli schiavi neri che cercavano di fuggire dalle piantagioni americane. Sempre dai Paesi Bassi, l'editore Everhardus J. Potgieter, amico e benefattore di Tine, aveva inviato a Stéphanie insieme ad una sua lettera la copia della biografia di Conrad Jan Hacke van Mijnden (1814-1873), teologo e studioso di letteratura italiana e provenzale, che aveva dedicato la sua vita a Dante (del quale tradusse in olandese la Commedia, 1867; 1870; 1873), Petrarca e Tasso. Van Mijnden morì un anno prima di poter partecipare al tanto atteso centenario petrarchesco (1874), con numerose celebrazioni a Padova e nella piccola villa di Arquà, dove i coniugi Omboni avrebbero incontrato lo stesso Potgieter (Masiero 2020, 413).11

Con l'inizio della sua attività a favore dell'infanzia e la partecipazione al comitato per la fondazione dei Giardini dell'infanzia (Kindergarten), Stéphanie Omboni lesse le opere di Friedrich Fröbel, così come il pamphlet Plus d'animaux (1886), opera dell'attivista per i diritti degli animali Mathilde Kleinmann Van Eys, in occasione della fondazione a Padova la Società Zoofila (1895). La Van Eys, trasferitasi a Sanremo al seguito del marito, si sarebbe cimentata nella traduzione de Il mio ultimo amico, romanzo di Edmondo De Amicis (Mon dernier ami. Traduit de l'Italien par Mathilde Van Eys, avec l'autorisation de l'auteur, 1903). Le sue frequenti lettere a Stéphanie Omboni, le segnalazioni e i dossiers delle sue visite ai diversi ricoveri per animali randagi in Europa, vennero pubblicate nella rivista The Zoofilist, come il reportage $A$ visit to the Homes for stray animals in Paris, Amsterdam, The Hague and Geneva del 1884 (Masiero 2020, 451-5).

10 Lettere del 17 ottobre 1863 e del 26 novembre 1863.

11 Lettera di Stéphanie Omboni a Everardus J. Potgeiter, 1873, 26 novembre: «La vostra gentile lettera e l'interessante articolo del vostro amico mi hanno dato una piacevole sorpresa; come avrete notato, le nostre lettere si sono incrociate, il che deve farmi sembrare più scusabile ai vostri occhi. Abbiamo letto con grande interesse la biografia di Haacke van Mijnden, scritta con tanto cuore ed entusiasmo - riteniamo che ogni pagina ispira l'affetto e i grandi meriti dell'eminente uomo, quale il suo soggetto». 


\subsection{Paolo Mantegazza}

Tornando nel contesto italiano, uno dei più prolifici interlocutori della famiglia Omboni fu l'antropologo Paolo Mantegazza, amico e compagno di studi di Giovanni, nonché grande estimatore di Stéphanie, con la quale intrattenne una breve corrispondenza trattando temi legati alla sua attività filantropica. ${ }^{12}$ La madre di Mantegazza era la filantropa milanese Laura Solera (1813-1873), ispiratrice dell'associazionismo femminile e maestra di due delle più importanti apostole della beneficienza dell'Italia unita, Ersilia Bronzini Majno e Alessandrina Ravizza..$^{13}$ Il Mantegazza nutriva una grande venerazione per la madre, della quale scrisse una biografia, La mia mamma (1876), che divenne un testo di riferimento, quasi un'agiografia, tra le mani di molte emancipazioniste del secondo Ottocento.

L'intento celebrativo delle grandi donne sue contemporanee spinse Paolo Mantegazza a voler dedicare un fascicolo del suo Almanacco igienico-popolare alla moglie del suo migliore amico, Stéphanie. L'Almanacco, che metteva a disposizione della prassi il sapere scientifico per la salute e l'igiene pubblica (cura del corpo, igiene della casa, pulizia della cucina, prevenzione delle malattie), era uno strumento importante per le Leghe della moralità pubblica, che avevano come obiettivo la diffusione di una cultura del bene comune tra le classi sociali più disagiate, e Stéphanie Omboni rappresentava nel pensiero del Mantegazza un'eccellenza in questo campo (Marciano 2004, 82-4). In una lettera del 23 novembre 1896, indirizzata all'amico Giovanni Omboni, Paolo Mantegazza chiese alcune informazioni sull'attività filantropica della moglie Stéphanie, dichiarando la sua inten-

12 Dall'Inventario (Frati 1991) si possono trarre alcune minute interessanti dell'epistolario con Giovanni Omboni, indirizzate anche a Stéphanie: nr. 632 (1873, 23 febbraio), nr. 685 (1874, 14 dicembre) su di un elisir per Stéphanie; nr. 734 (1875, 23 ottobre) il Mantegazza si allontana dalla città in campagna per alcuni disturbi di salute; nr. 793 (Paolo Mantegazza ai coniugi Omboni, 1876, 27 dicembre) il Mantegazza descrive con toni divertenti il Senato. Tra le lettere conservate nel Fondo fiorentino, si trovano anche le lettere inviate dalla sola Stéphanie Omboni: nella nr. 962 (1881, 13 gennaio) e nella successiva, la nr. 973 (1881, 2 aprile) la Omboni raccomanda a Mantegazza il professor Giovanni Weiss, patologo trentino, all'epoca in cattedra a Ferrara, per l'Università di Catania. Nella lettera del gennaio ‘81 Stéphanie Omboni chiede la disponibilità di Mantegazza per una conferenza a Padova. Infine, nella lettera nr. 976 (1881, 15 aprile) Stéphanie Omboni invia all'amico quattro illustrazioni. Lo scambio epistolare, dopo un silenzio documentario di quindici anni - tale da far supporre che le lettere siano andate perdute - riprende nel 1906: nella lettera nr. 2558 (1906, 12 luglio) Stéphanie Omboni scrive a proposito dell'epigrafe commemorativa di Laura Solera, mentre qualche giorno dopo, nella lettera nr. 2559 (1906, 15 luglio) si dice dispiaciuta per la malattia del Mantegazza. In un'ultima lettera, la nr. 2675, non datata ma dell'8 novembre, la Omboni riporta all'amico i giudizi della di lui figlia, Laura (Laurina) Mantegazza Polcari.

13 Le lettere ricevute dal Mantegazza e le minute della corrispondenza inviata sono riportate in regesto in Frati 1991. Si veda anche Capannelli, Insabato 1996; Ehrenfreund 1926; Chiarelli, Pasini 2010. 
zione di volerle dedicare l'Almanacco del 1897 successivo (Frati 1991, 197). A margine della lettera, l'Omboni annota che il 5 dicembre aveva restituito a Mantegazza le bozze corrette della dedica, ma che il giorno successivo, con un poscritto, gli inviava una sua nota sull'opportunità di pubblicarla, dimostrando al Mantegazza la tempra della donna: era noto che Stéphanie vivesse con disagio e ritrosia ogni forma di omaggio per la sua attività di filantropa e benefattrice, preferendo rimanere nelle retrovie, quasi nascosta. Non avendo altra possibilità che accettare le volontà dell'amica, Paolo Mantegazza pubblicherà l'Almanacco con la dedica a Giovanni Omboni.

Nel mazzetto di lettere rinvenuto tra gli effetti di Stéphanie Omboni e oggi conservato presso la Biblioteca Universitaria di Padova, Paolo Mantegazza racconta all'amica le proprie fatiche editoriali, alle quali la donna sembra partecipare con pareri e contributi puntuali. Nel 1899, ad esempio, Mantegazza inviò a Stéphanie tre copie dei Caratteri regionali, preludio di un'opera successiva. ${ }^{14}$ In una seconda lettera, infatti, parlerà alla Omboni del «lungo martirio di gestazione» de I caratteri umani, opera divulgativa che univa l'antropologia alla fisiologia, secondo uno sperimentalismo di frontiera che aveva molti tratti in comune con il behaviourismo lombrosiano, e nel capitolo dedicato agli Altruisti riesce a collocare finalmente la Omboni accanto alla memoria della madre, Laura Solera: «Questo è il vero altruismo, quello che ha sentito la mia santa mamma, quello di cui è alta sacerdotessa la mia illustre amica Stefania Omboni». ${ }^{15}$

Impegnato prima nell'insegnamento e poi come senatore del Regno, Mantegazza impiegherà lungo tempo per portare a termine $L a$ Bibbia della Speranza (1910), l'opera dove ha «condensato - scrive alla Omboni - quanto di vero e di sano pensarono gli uomini». ${ }^{16}$ Man-

14 BU, ms. provv. 217, c. 1: «Felicissimo che il mio povero libretto sui Caratteri regionali vi sia piaciuto, ve ne mando tre copie in omaggio delle vostre indulgenze per un lavoro che giudico modestissimo e del quale quasi mi vergogno» (Lettera di Paolo Mantegazza a Stéphanie Omboni, 1899, 23 gennaio).

15 BU, ms. provv. 217, c. 6: «Sto correggendo le bozze del mio libro I caratteri umani, che dopo un lungo martirio di gestazione, finalmente sta per venire alla luce; e nel capitolo degli "Altruisti" leggo queste parole che desidero siano lette da voi prima che dal pubblico:... (sic!) «Questo è il vero altruismo, quello che ha sentito la mia santa mamma, quello di cui è alta sacerdotessa la mia illustre amica Stéphanie Omboni. Questo è l'altruismo che va a traverso della ragione, e che deve prendere il posto della carità manipolata ad un del purgatorio e dell'inferno dei frati di un medioevo che ancora non è morto». Spero che questo mio nuovo libro vi piacerà. Contemporaneamente se ne sta stampando un altro, Il libro delle malinconie. Addio, e abbracciatemi caramente il nostro Blas. Vostro, Mantegazza». (Lettera di Paolo Mantegazza a Stéphanie Omboni, 1901, 31 maggio). Si veda a tal proposito anche Mantegazza 1876.

16 Mantegazza 1909. Nella lettera di Paolo Mantegazza a Stéphanie Omboni, 1907, 2 febbraio (BU, ms. provv. 217, c. 13r), la Bibbia della Speranza è definita dal Mantegazza il suo «opus magnum»; nelle lettere del 1908, 14 febbraio (BU, ms. provv. 217, c. 15r) e del 1908, 29 aprile (BU, ms. provv. 217, c. 18r) è «quasi finita»; nel 1908, 23 giugno 
tegazza, fin dalle sue lettere del 1907, ebbe a cuore di aggiornare Stéphanie sul processo di scrittura dell'opus magnum, come ebbe a definire la summa delle sue ricerche sull'igiene pubblica a servizio della moralizzazione della società. Mantegazza torna così sui temi trattati nell'Almanacco, non senza le critiche di chi, come Benedetto Croce, riteneva le sue ultime pubblicazioni come una fragile commistione di scienza e letteratura, senza riuscire compiutamente né nell'una né nell'altra (Pireddu 2010, 188). ${ }^{17}$ Tuttavia, l'aspirazione di compendiare in un'unica grande opera le numerose ricerche sulla critica sociale e umanitaria gli valse il plauso di molte delle donne impegnate nella moralizzazione dei ceti disagiati, tra le quali la stessa Omboni. ${ }^{18}$

Ancora due opere vennero sicuramente lette da Stéphanie Omboni, una di carattere storico-autobiografico, A 16 anni sulle barricate di Milano (1900), ${ }^{19}$ scritta dal Mantegazza in occasione della pubblicazione della prima cronaca corale del Risorgimento italiano, e una di filosofia critica positiva, l'Elogio della vecchiaia (1894), ${ }^{20}$ nella quale Mantegazza cercava di dimostrare come la vecchiaia non rappresentasse il deterioramento dell'uomo ma una forma di energia inespressa. La vecchiaia era per Mantegazza la stagione feconda della felicità, della bellezza e del genio, dove l'uomo raggiungeva la pienezza della conoscenza della Natura e della Storia. Ogni vecchio era un vero e proprio 'filosofo della natura' - sosteneva Mantegazza - perché riusciva a percepire il cuore del mondo, dell'etica e della vita stessa (i concetti e le espressioni metaforiche che compaiono nel romanzo sono comuni a Nietzsche e Rousseau). A riprova della fiducia che nutriva nella sua intuizione, nelle lettere in cui men-

(BU, ms. provv. 217, c. 19r), Mantegazza scrive: «In questi giorni ho terminato la mia Bibbia della speranza, ma mi rimane la parte più difficile, trovare l'editore». Trascrive la dedica che comparirà in esergo, in anteprima per gli Omboni (l'aveva tenuta segreta persino alla moglie, Maria): "Ai centomila che credono a tutto, non escluso l'assurdo, ai mille infelici che non credono a nulla dedico questa Bibbia della speranza nella quale ho condensato quanto di vero e di sano pensarono gli uomini da Budda e da Cristo a Dante e da Dante a Darwin».

17 La critica alla fisionomia dello scienziato-letterato viene esposta in Croce 1957.

18 Armeniese 2017, 25: «la migliore igiene della salute possa essere esercitata per il tramite della morale e, per questo, vi riporta un singolare bilancio dell'Igiene della morale caratterizzato da fattori attivi (il lavoro, la castità, la temperanza, la bontà, l'economia di tutte le forze) e passivi (l'ozio, il libertinaggio, l'intemperanza, la malvagità, lo scialacquo, l'ambizione eccessiva, la vanità); seguendo scrupolosamente i principi scatenanti del bilancio attivo della suddetta «igiene», considera che un soggetto possa pervenire alla piena felicità, ispirata proprio dalla «sanità» del corpo e dalla conduzione di una vita igienica (che ben ci dispone ad essere "buoni e cortesi")».

19 Mantegazza 1900, citato nella lettera di Paolo Mantegazza a Stéphanie Omboni, 1899, 23 febbraio (BU, ms. provv. 217, c. 2v).

20 Lettera di Paolo Mantegazza a Stéphanie Omboni, 1908, 18 febbraio (BU, ms. provv. 217, c. 17r). 
zionava l'opera Mantegazza contava la sua età a partire dal giorno in cui aveva pubblicato l'Elogio, quasi a voler dimostrare alla sua interlocutrice di prendere sul serio la sua teoria della 'nuova nascita' nel tempo della vecchiaia. ${ }^{21}$

\subsection{Rina Faccio/Sibilla Aleramo}

Il rapporto tra Stéphanie Omboni e Rina Faccio/Sibilla Aleramo si sviluppa e si consuma nell'arco di quasi quindici anni, tra il 1899 e il 1915, dal primo incontro avvenuto tra le due a Milano, proseguito attraverso un intenso rapporto epistolare recentemente riportato alla luce (Vozza 2020). Oltre al tratteggio del rapporto intimo e profondo tra le due donne, tra le pieghe della corrispondenza si possono ricavare informazioni sui libri letti da entrambe, fino alla pubblicazione di Una donna (1906), il romanzo autobiografico di Rina/Sibilla che costituirà per l'autrice il crinale da oltrepassare verso la maturità. Quelle lettere sono documenti che raccontano le diverse iniziative delle femministe italiane in favore della causa emancipazionista, dagli interventi sui periodici ai convegni, fino ai racconti più toccanti sulle difficoltà coniugali vissute da Rina col marito, Ulderico Pierangeli, e la relazione amorosa con lo scrittore Giovanni Cena.

Il tenore delle lettere scritte dalla Omboni lascia trasparire in controluce una tensione spirituale che attingeva all'esistenzialismo profetico condiviso da scrittori e filosofi del tardo Ottocento italiano, e che troverà in Giovanni Cena ed Gaetano Meale i due interlocutori privilegiati. La consapevolezza dell'esistenza di anime elette, di intelletti superiori, è un tema ricorrente nel carteggio. In particolare, la Omboni è fortemente influenzata dalla lettura di alcuni esiti della letteratura esistenzialista, esperienza che consiglia anche all'amica (Vozza 2020, 97-8). ${ }^{22}$ Il primo libro, citato nello scambio con la Aleramo semplicemente come Journal d'Amiel, è il celebre Fragments d'un journal intime, il diario del filosofo ginevrino Henri-Frédéric Amiel

21 «E mi pento d'aver scritto l' Elogio della vecchiaia, libro che fu tradotto in portoghese, ma che è una sola lingua. L'ho scritto quando mi credevo vecchio (a 65 anni) ma non conoscevo la vecchiaia vera». Così Paolo Mantegazza nella lettera a Stéphanie Omboni, 1909, 9 marzo (BU, ms. provv. 217, c. 20v), mentre nella lettera del 1909, 17 giugno (BU, ms. provv. 217, c. 21r) Mantegazza dichiara di aver scritto l'Elogia all'età di 62 anni.

22 «Je voudrais vous envoyer un livre qui doit être en harmonie avec votre état d'esprit en ce moment, si vous ne l'avez pas déjà lu: "Le journal d'Amiel"? Connaissez-vous aussi un autre livre : "Les grand initiés"?» (Lettera di Stéphanie Omboni a Rina Faccio, 23 settembre 1901) e ancora: «Voyez-vous, notre Umano se trouve dans le cas de tous les grands esprits (les "grands Initiés" comme dit Schuré, voilà un livre que je vous prêterai lorsque vous aurez lu Amiel) depuis que le monde existe» (Lettera di Stéphanie Omboni a Rina Faccio, 2 novembre 1901). 
(1821-1881), scritto sotto forma di lettere ad un altro-da-sé e pubblicato postumo (1884). Insieme a questo, la Omboni consiglia la lettura de Les grands initiés, un saggio di storia delle religioni scritto nel 1889 da Édouard Schuré (1841-1929) il cui titolo originale, Les Grands Initiés. Esquisse de l'histoire secrète des religions. Rama, Krishna, Hermès, Moïse, Orphée, Pythagore, Platon, Jésus, delinea un percorso filosofico e iniziatico dei grandi pensatori e "profeti" dell'antichità alle verità ultime dell'esistenza, affine ai capisaldi della teosofia, dottrina alla quale la Omboni si era avvicinata negli anni Ottanta. Uno degli estimatori più entusiasti di Schuré fu proprio Dino Campana, amante e compagno di Sibilla Aleramo, che riverserà la lezione misterica dello scrittore insieme alla speculazione nietzschiana sull'apollineo e il dionisiaco nei suoi Canti orfici (1914). Anche Rina/Sibilla racconterà di aver letto e riflettuto sulle parole di Amiel, come scriverà nel romanzo Una donna, forse proprio accogliendo l'invito della Omboni di edificarsi con la profondità dei contenuti di quel saggio. ${ }^{23}$

Il libro che ha profondamente segnato la spiritualità laica di Stéphanie Omboni fu Gli Ammonitori, scritto da Giovanni Cena. ${ }^{24} \mathrm{Du}-$ rante la permanenza in Carinzia presso la sorella, nell'agosto del 1904, la Omboni scriveva alla Faccio di avere con sé una copia del romanzo, un libro «profondément pensé, profondément senti et vu, est sorti de ce grand cœur et de cette plume d'or» (Vozza 2020, 110-11). ${ }^{25}$

23 Aleramo 2003, 151: «Sfogliavo in quei giorni con una strana voluttà il Giornale intimo di Amiel. Fantasmi popolavano il mio studio, mi apparivano dinanzi fra le piante del giardino o in mezzo alle vie maestre o in riva al mare: mia madre giovane accanto alla culla delle mie sorelle, in atto d'accettare la sua sorte atroce; questo filosofo ammalato, curvo sulla sua scrivania ad esprimere il suo dolce pessimismo intessuto di lagrime di ruggiti repressi; un famoso scrittore nostro, infine, una delle mie ammirazioni d'adolescente, a cui poco innanzi il figlio ventenne era morto, suicida, vittima forse del dissidio tra i genitori. Simboli sanguinosi della vanità del sacrificio, esempi terribili del castigo incombente su ogni coscienza che si rinnega. Non ero io una di queste coscienze?».

24 Cena 1904, 136: «Ho trovato per gli altri la ragione di vivere e per me la ragione di morire. Il suicidio è viltà quando significa fuga. Io non fuggo. Io m'immergo nella vita: io dò certamente, colla mia morte, comunque fruttifichi, e il sacrifizio non è mai senza frutto, un maggior senso di libertà e di solidarietà ai miei simili. Io dico a chiunque viva oggidì, posto dalla società in qualsiasi condizione: "Bisogna operare e amare. Bisogna limitare il proprio volere, congiungerlo in armonia col volere dei nostri simili. Umile o precipua parte nel coro sociale, espandi in tutta la sua potenza l'anima che ti diede il destino, abbraccia l'umanità e la natura, compenetrati e fortificati di esse: opera ed ama!"». Sulla ricezione del romanzo, si veda il recente Risso 2020.

25 «Quant à moi j'ai une vie très-prise, très-absorbante à Padoue, une occupation s'enchaine à l'autre, au point que, vous ne le croirez pas, je n'arrive jamais à ouvrir un livre. En voilà une ignorante ! Une illettrée ! J’ai avec moi "Gli Ammonitori” que je vais dévorer avec enthousiasme. Naturellement j'ai lu dans les journaux une foule de critiques, qui me disent d'avance quel grand livre, profondément pensé, profondément senti et vu, est sorti de | ce grand cœur et de cette plume d'or. A propos de livre. Je cherche désespérément un livre auquel je tiens tant tant, et que je ne trouve plus dans ma bibliothèque. A qui puis-je l'avoir prêté ? Pas à vous, j'en suis sure, mais si je me trompais, dites-le-moi. Et surtout dites-moi de vous, de l'ouvrage dans lequel vous voulez mettre 
L'opera di Cena era frutto di una puntuale riflessione sui cambiamenti in atto nella società torinese a cavallo dei due secoli, inserita in un filone saggistico-narrativo che già aveva fatto la fortuna di scrittori come Charles Dickens, Emile Zola e Giovanni Verga. I temi affrontati dagli autori del romanzo sociale che rimarranno ai margini della letteratura italiana, come Cena, includono la povertà, le condizioni nelle fabbriche e nelle miniere, la difficile situazione del lavoro minorile, la violenza contro le donne, l'aumento della criminalità e le epidemie a causa del sovraffollamento e le scarse condizioni igieniche nelle città. Per la Omboni un'opera come Gli Ammonitori diventava il rifugio spirituale per coloro che avvertivano l'urgenza di una presa di coscienza collettiva sulle ingiustizie sociali, ma nello stesso tempo viveva il paradosso nietzschiano dell'indifferenza dell'umanità alle profezie delle anime elette, degli 'ammonitori'. Meno di una settimana dopo la prima lettera, la Omboni scriveva nuovamente all'amica, il 20 agosto 1904. I toni dello scritto sono entusiasti, quasi euforici, come se il romanzo avesse restituito alla benefattrice inglese un modello di riferimento nel quale identificare sé stessa e la propria opera filantropica: «L'ho letto. Quanto pensiero e quanta poesia in quel libro. L'autore ha saputo dar forma all'intima essenza delle cose e degli esseri [...], vi fa intravvedere l'inesprimibile, il vero fondo delle cose, il solo vero» (Vozza 2020, 112).

Un entusiasmo non dissimile, due anni più tardi, fu espresso dalla Omboni dopo la lettura del romanzo Una donna. La forza della testimonianza autobiografica, avvolta nell'anonimato e scritta sotto forma di romanzo, diventa la chiave «fin troppo scrupolosa e personale, artisticamente parlando» ma necessaria «all'effetto di rappresentare una esistenza in tutta la sua causalità, in tutte le sue affinità». E così, nella lettera del primo marzo 1907: «Oh! che libro doloroso e potente! - scrive la Omboni - Anche chi non lo sa deve sentire che è una pagina di vita vissuta» (Vozza 2020, 116-17). ${ }^{26}$ L'apprezzamento per il romanzo andava di pari passo con la certezza di sapere Rina/ Sibilla confortata dal ristoro «di vivere in un ambiente puro ed elevato, sostenuta dall'affetto ideale di un'anima buona e devota, da una

toute votre âme, toute la fleur de votre pensée et de votre cœur. Encore et encore je vous remercie pour votre belle chère lettre du 5 Avril, que je relis en ce moment» (Lettera di Stéphanie Omboni a Rina Faccio, 13 agosto 1904).

26 "Sono stata fra le primissime a leggere "Una donna”, appena uscito lo lessi ad alta voce a mio marito, senza, si intende, svelare né a lui né a nessuno il nome dell'autrice. Lo lessi con passione, con una emozione profonda, indicibile, seguendo ogni fase di quella esistenza dolorosa già da me conosciuta, colla Sua cara persona sempre innanzi agli occhi, tale l'ho conosciuta nei primi anni a Milano. È terribile di verità, di analise coraggiosa e franca, e da questa verità scaturiscono la protesta energica, veemente contro questo crudele sacrificio della donna, schiava a casa, l'appello fremente in favore della madre. Oh! che libro doloroso e potente! Anche chi non lo sa deve sentire che è una pagina di vita vissuta» (Lettera del 1907, primo marzo). 
intelligenza superiore!». Stéphanie Omboni con queste parole allusive si riferiva a Giovanni Cena, la cui presenza nella vita di Rina aveva permesso la sua emancipazione umana ed artistica, a prezzo tuttavia di alcune rinunce e censure (come quella relativa alla vita di Rina prima del suo incontro con lui).

\subsection{Gaetano Meale, alias Umano}

Figura controversa del panorama intellettuale italiano di fine Ottocento, lo scrittore Gaetano Meale (1858-1927) meglio noto con lo pseudonimo di Umano, fu un magistrato, filosofo e libero pensatore, nonché uno dei più decisi assertori di un movimento pacifista internazionale. Meale godette della stima e dell'amicizia di alcune personalità non secondarie del movimento emancipazionista, come Alessandrina Ravizza e la stessa Rina Faccio, che lo inserirà nel romanzo Una donna sotto le vesti anonime del 'profeta' (Vozza 2020, 78-84). Nella corrispondenza tra Stéphanie e Rina, Umano compare in dodici delle trentacinque lettere, in molte delle quali è protagonista indiscusso. Più di Gaetano Meale, uomo fragile e nevrastenico (Minuto 2017, 138), benché sospinto da nobili e alti ideali, è l'alias dell'intellettuale e mistico Umano che guadagna la fiducia quasi discepolare delle due donne. In una delle sue lettere, ad esempio, Stéphanie descrive punto per punto il lungo e travagliato percorso di gestazione, scrittura e pubblicazione di Fede eterea (1903-1906), il manifesto del pensiero di Umano, sottoscritto da alcuni degli intellettuali più eminenti del suo tempo. In una lettera del primo febbraio 1902 la Omboni scrive alla Faccio che il nucleo centrale dell'opera è ben evidente fin dalla stesura embrionale che ha potuto leggere del primo volumetto, Antifona e sinfonia, tanto ricco di idealismo da esporsi alle critiche più severe dei suoi detrattori.

Oltre a Fede eterea, Stéphanie dimostra di aver letto le due opere sul pacifismo, Fine delle guerre (Meale 1889) e Patria Lex (Meale 1900). ${ }^{27}$ Mentre nel 1889 si celebrava la Seconda Internazionale e a Parigi si teneva il primo Universal Peace Congress, in Italia prendeva forma il movimento continentale per la promozione dell'arbitrato e la pace internazionale. Gli sforzi della compagine socialista e di quella borghese andavano nella direzione della cooperazione, ambendo al risultato più significativo del disarmo multilaterale. È in questo con-

27 Si legge nella lettera di Stéphanie Omboni a Rina Faccio, 16 gennaio 1901: «La società del nostro caro Umano deve essere un vero conforto per Lei. Quando lo vedrà gli dica che l'ho nel cuore, che sto per scrivergli e che mi dà tanto piacimenti il suo "Patria Lex", la prima parte, quella seria, esposta con una chiarezza di concetto, una fermezza di linea ben superiore alla "Fine delle guerre", la seconda parte di una ironia sanguinosa, magnifica» (Vozza 2020, 91). 
testo, gravido di aspettative, che Meale scrive La fine delle guerre, una critica radicale all'approccio paternalistico dei liberal-democratici, indicati dall'autore come i responsabili di quegli stessi nazionalismi che spingevano ai confini degli stati e soffiavano venti di guerra.

Ma è nel successivo Patria Lex che Umano tende ad assolutizzare le tesi del suo precedente scritto (che, nel frattempo, era arrivato alla terza edizione nel 1896) e che costituiva una nuova riflessione sul pacifismo internazionalista. Lo stile, volutamente sarcastico, teorizzava la fratellanza universale e l'instaurazione di una società che si fondava sul socialismo cristiano. L'ordine internazionale, secondo Meale, doveva essere tuttavia condotto dai paesi più civili, gli unici ai quali era possibile utilizzare la forza per ripristinare la pace (le vicende della guerra anglo-boera, infatti, furono l'occasione della scrittura di Patria Lex). Insoddisfatto degli esiti dell'assemblea tenuta all'Aja che portò all'omonima convenzione (un'assemblea ritenuta poco più di una vana consolazione), lasciò la magistratura, dedicandosi alle sue speculazioni filosofiche.

\section{Conclusioni}

A conclusione di questa rassegna si possono ricavare alcune informazioni che vanno ad arricchire la biografia di Stéphanie Omboni, definendo il milieu culturale e le relazioni intessute dalla donna con la società intellettuale del suo tempo. L'analisi quantitativa e qualitativa basata su di un campione accidentale di libri superstiti, è bene ricordarlo, offre soltanto uno spaccato della realtà materiale che si è voluta esporre in questo saggio. Tuttavia, è interessante considerare, almeno sotto il profilo metodologico, che i trenta pezzi librari conservati presso il Museo dell'Educazione dell'Università di Padova fotografano un processo di selezione avvenuto a posteriori: quelle giunte fino ad oggi sono opere che non vennero portate via dagli amici dei coniugi Omboni - Albertotti, Buzzacarini, Bianchi, e Dal Piaz - e che andarono ad arricchire la Biblioteca di Geologia o gli scaffali delle loro librerie private. Sono opere scartate e che, dunque, non destarono la curiosità o l'interesse scientifico, né vennero stimate per il loro pregio o la rarità. Esse riconsegnano l'immagine del quotidiano, di una semplicità fatta anche di guide turistiche e romanzi, di dizionari e saggi storici, rappresentative - e questo è un tratto insolito nella ricostruzione delle biblioteche borghesi dell'Otto-Novecento - di una ordinarietà spesso ignorata in favore della ricerca di opere ben più significative e diffuse, che con la loro presenza confermano il trait d'union delle élite intellettuali.

Così come Giovanni Omboni si recava al gabinetto di lettura per leggere i quotidiani e periodici, anche Stéphanie Omboni acquistava e leggeva molte riviste, in particolare quelle diretti dalle eman- 
cipazioniste italiane, dal giornale La Donna che lei stessa contribuì a fondare a Padova fino all'Unione femminile, o ancora periodici che godettero di una certa fortuna grazie ai loro direttori, come la Nuova Antologia sotto la direzione di Giovanni Cena. In solidarietà con i comitati femministi dei paesi vicini, Stéphanie Omboni si abbona - e consiglia gli amici di fare altrettanto - ai periodici inglesi e tedeschi (ben più avanzati in tema di diritti delle donne) o francesi, come nel caso del foglio parigino La Fronde, diretto da Marguerite Durand (Vozza 2020, 62).

Il pragmatismo, tratto distintivo di Stéphanie Omboni, si riflette anche sul trattamento riservato alle pagine di quei libri: il tempo e le energie riversate nella beneficienza, nell'associazionismo o nella filantropia (tempo sottratto, per sua stessa ammissione, allo studio e alla lettura, tanto da definire sé stessa una «illettrée») richiese uno sforzo non indifferente per lo studio dei codici di leggi per stendere statuti e regolamenti in punta di diritto. Ecco perché dal punto di vista estrinseco il libro che mostra i segni più evidenti di usura è la Nuova legge sulle opere pie illustrata da brevi cenni intorno alle sue origini ed ai suoi motivi. Susseguita dai regolamenti del 1891. A contemperare la tensione al pragmatismo radicale vi era una costante ricerca di ristoro nella lettura di romanzi filosofici e lato sensu spirituali, come Gli Ammonitori di Cena, il Diario intimo di Amiel o studi critici sul cristianesimo e la figura di Gesù Cristo. La religiosità di Stéphanie Omboni è un tema controverso (Masiero 2020, 54-5), che certamente non trova in questo saggio una risposta risolutiva; tuttavia, la filantropia vissuta come missione laica verso l'umanità emarginata, conserva in sé stessa i tratti di quella tensione verso la condivisione delle prospettive universaliste dei valori intrinseci della religione, come l'amore, la fratellanza, il soccorso dei più deboli. Il terreno su cui si muove Stéphanie Omboni è quello social-liberale della borghesia italiana del Risorgimento, anticlericale ma non antireligiosa, in cui la fede diventava una questione privata, ma i principî morali un patrimonio comune.

Infine, in alcuni passaggi delle lettere prese in esame in questo saggio si legge che Stéphanie Omboni inviava ai suoi interlocutori non solo suggerimenti o dattiloscritti, ma dava in prestito anche i libri di sua proprietà, divenendo essa stessa la prima artefice della dispersione della sua biblioteca. Così scrive in una lettera a Rina Faccio il 13 agosto 1904: «A propos de livres. Je cherche désespérément un livre auquel je tiens tant tant, et que je ne trouve plus dans ma bibliothèque. A qui puis-je l'avoir prêté ? Pas à vous, j'en suis sure, mais si je me trompais, dites-le-moi» (Vozza 2020, 114). Stéphanie Omboni ebbe l'occasione di leggere in anteprima alcune opere e dare il suo personale contributo, come avvenne per I caratteri umani o La Bibbia della Speranza di Mantegazza, Antifona e sinfonia di Gaetano Meale o i pamphlet femministi di Rina Faccio. Ma fu ella stessa autrice di 
articoli di giornale e saggi, oltre che traduttrice, confrontandosi via lettera con il marito Giovanni e con le amiche Alessandrina Ravizza, Ersilia Majno Bronzini e Filomena Cuman Fornasari: più di una dozzina di rendiconti morali delle associazioni delle quali era consigliera o vicepresidente (nonché occulta fondatrice), relazioni di convegni e un saggio monografico Sur un système plus efficace pour la protection des enfants pauvres et malheureux (1910).

Con il proseguire della ricerca, in particolare nei fondi ancora oggi parzialmente inesplorati, presso il Museo dell'Educazione dell'Università di Padova o il Dipartimento di Geoscienze - dove si trovano, depositati, molti documenti personali di Giovanni Omboni - si auspica di poter rinvenire in futuro ulteriori informazioni utili a completare il quadro generale delineato in questo studio introduttivo. 


\section{Appendice. La biblioteca di Stéphanie Omboni}

1

The Soul of a Priest

Duke Litta [alias Litta Visconti Arese, Pompeo].

London, T. Fischer Unwin, 1907.

2

A New Complete Dictionary of English and German Languages

[Kaltschmidt Jacob Heinrich].

Leipsic, Printed for Charles Tauchnitz, 1849

3

A Complete Practical Grammar of the German Language

[Schade, Charles Benjamin].

Leipzig, Printed for J. C. Henricus $1853^{6}$

4

Albertino Mussato. Studio storico e letterario

Zardo, Antonio.

Padova, Libreria Draghi, 1884

5

Guida Itinerario dell'Italia e di parte dei Paesi limitrofi

Seconda parte, vol. III: Lazio

Bertarelli, Luigi Vittorio.

Milano, Touring Club Ciclistica Italiana, 1897

6

Nouvelle Grammaire française sur un plan très-méthodique avec de nombreux exercices d'orthographe, de syntaxe, et de ponctuation

[Noël, François-Joseph-Michel; Chapsal, Charles-Pierre].

Paris, Maire Nyon-Roret-Hachette, 1886.

\section{7}

Istituzioni pubbliche di beneficenza. Nuova legge sulle opere pie illustrata da brevi cenni intorno alle sue origini ed ai suoi motivi. Susseguita dai regolamenti Milano, Edoardo Sonzogno Editore, 1891

8

Esoteric Christianity or the Lesser Mysteries

Annie Besant.

London \& Benares, The Theosophical Publishing Society, 1901 
9

Aux mères de famille. L'Ecole de la Pureté, parMme E. Pieczynska

[Emma Reichenbach Pieczynska]

Paris, Librairie Fischbacher, [1902 $\left.{ }^{4}\right]$

10.1

Schillers Sämmtliche Werke in zwölf Bänden

Zweiter Band [2]

[Schiller, Johann Christoph Friedrich]

Stuttgart und Tübingen, Gotta'scher Verlag, 1847

10.2

Schillers Sämmtliche Werke in zwölf Bänden

Vierter Band [4]

[Schiller, Johann Christoph Friedrich]

Stuttgart und Tübingen, Gotta'scher Verlag, 1847

\section{3}

Schillers Sämmtliche Werke in zwölf Bänden

Achter Band [8]

[Schiller, Johann Christoph Friedrich]

Stuttgart und Tübingen, Gotta'scher Verlag, 1847

10.4

Schillers Sämmtliche Werke in zwölf Bänden.

Zwölfter Band [12]

[Schiller, Johann Christoph Friedrich]

Stuttgart und Tübingen, Gotta'scher Verlag, 1847

11

Jérome Paturot à la recherche d'une position sociale. Par Louis Reybaud. Auteur des Etude sul les Reformateurs ous Socialistes modernes

Tome premier

[Reyabud, Louis]

Paris, Paulin Editeur, 1847

12

New Italian and English Dictionary in two parts

English-Italian. Italian-English.

[F.C. Meadows].

London, Printed for Thomas Tegg \& Son, 1836

13

Italie et Sicilie par P. Joanne. 4 cartes et 12 plans

[Joanne, P.]

Paris, Librairie Hachette et C. ${ }^{\text {ie }}, 1888$ 
14

Les Avventures de Jean-Paul Choppart, reccuilles et racontées a ses jeunes amis par

Louis Desnoyers

Tome premier (quarta ed.).

[Desnoyers, Louis]

Malines et Bruxelles, P. J. Hanicq et C. ${ }^{\text {ie }}$ Editeurs, 1836

15

Early Lessons by Maria Edgeworth.

A New Edition collected into one volume.

[Edgeworth, Maria].

London, Published by Longman Brown \& Co., 1857.

16

Primo libro di lettura e conversazione tedesca

Terza edizione.

Massimo Grünhut.

Torino [et alii], Ditta G. B. Paravia e C., [1902]

17

New English and Italian Dictionary.

By John Millhouse. Third edition. Volume I. With many additions by Ferdinand Bracciforti.

[Millhouse, John].

Milan, Printed for the heirs of the Author, 1864.

18

Nouvelles narrations françaises précédés d'exercices préparatoires par M. Filon (settima ed.).

[Filon, M.]

Paris, Librairie de L. Hachette et C. ${ }^{\text {ie }}, 1860$.

19

Vita di San Francesco di Sales, vescovo e principe di Ginevra. Compilata sui manoscritti e sugli autori contemporanei dal curato di S. Sulpizio di Parigi autore della vita del Card. di Cheverus (tradotta dal francese)

Volume II.

[Hamon, Andre-Jean-Marie].

Torino, Cav. Pietro Marietti, 1885.

20

Lezioni sulle leggi ed usanze del commercio inglese con note italiane per il Prof. Raffaele Gambaro della R. Scuola Superiore d'Applicazione per gli studi commerciali in Genova [Gambaro, Raffaele].

Genova, Tipografia del R. Istituto Sordo-Muti, 1890 
L'Ami des Enfants, ou L'Education à l'école et dans la famille. Directeur M. L. Roussel (partie littèraire).

Année 1878-1879.

[Roussel, Abbé M. L.].

Administration Rue La Fontaine 40, Paris Auteuil [1879]

\section{2}

Il Gabinetto di Geologia della R. Università di Padova

Giovanni Omboni.

Padova, Tipografia all'Università - Fratelli Gallina, 1898

\section{1}

Précis de la Géographie Universelle ou Description de toutes les parties du Monde sur un plan nouveau, d'après les grandes divisions naturelles du globe. Par Malte-Brun. Tome premier. Historie et théorie générale de la géographie.

[Malte-Brun, Conrad].

A Bruxelles, Chez les editeurs Berthot [et alii], 1829.

\section{2}

Précis de la Géographie Universelle ou Description de toutes les parties du Monde sur un plan nouveau, d'après les grandes divisions naturelles du globe. Par Malte-Brun. Tome second. Description de l'Asie, de l'Océanique et de l'Afrique Septentrionale. [Malte-Brun, Conrad].

A Bruxelles, Chez les editeurs Berthot [et alii], 1829.

\section{4}

Tine. Brieven van Mevrouw E.H. Douwes Dekker-van Wijnbergen aan Mejuffrouw Stephanie Etzerodt, later Mevrouw Omboni. Met een schrijven van de laatste en enkele aanteekeningen. Uitgegeven door Dr. Julius Pée.

[Pée, Julius].

's-Gravenhage, Martinus Nijhoff, 1895.

25

San famille. Par Hector Malot. Tome premier.

[Hector Malot].

Paris, E. Dentu Editeur, 1886.

\section{6}

The Englishwoman's Yearbook for 1887. And Directory to all institution existing fort the benefit of women and children. Corrected to date. By L. M. H. [Hubbard, Louisa Maria].

London, Hatchards Piccadilly, 1887. 


\section{Bibliografia}

Alaimo, A. (1991). «Le tipografie a Bologna nella seconda metà dell'Ottocento e il caso della Compositori». Berselli, A. (a cura di), Editoria e università a Bologna tra Ottocento e Novecento = Atti del quinto convegno (Bologna, 26-27 gennaio 1990). Bologna: Istituto per la storia di Bologna, 21-60.

Aleramo, S. (2003). Una donna. Milano: Feltrinelli.

Armeniese, G. (2017). "Sull'identità e funzione della pedagogia mantegazziana». D'Arcangeli Marco Antonio; Sanzo, A. (a cura di), Le "scienze umane" in Italia tra Otto e Novecento. Pedagogia, psicologia, sociologia e filosofia. Milano: Franco Angeli, 245-60.

Canadelli, E. (2015). «Un geologo si racconta: l'autobiografia inedita di Giovanni Omboni». Quaderni per la storia dell'Università di Padova, 48, 311-38.

Capannelli, E.; Insabato, E. (a cura di) (1996). Guida agli Archivi delle personalità della cultura in Toscana tra '800 e '900. L'area fiorentina. Firenze: Olschki, 359-61.

Cena, G. (1904). Gli Ammonitori. Roma: Nuova Antologia.

Chemello, A. (2002). «Lettura e lettrici nella tradizione letteraria italiana dell'Ottocento». Tortorelli, G. (a cura di), in Biblioteche nobiliari e circolazione del libro tra Settecento e Ottocento = Atti del convegno nazionale di studio (Perugia, 29-30 giugno 2001). Bologna: Edizioni Pendragon, 39-72.

Chiarelli, C.; Pasini, W. (a cura di) (2010). Paolo Mantegazza e l'Evoluzionismo in Italia. Nuova edizione. Firenze: Firenze University Press.

Croce, B. (1957). «Scienziati-letterati». La letteratura della nuova Italia, vol. 6. Bari: Laterza, 49-54.

Ehrenfreund, E. (1926). «Bibliografia degli scritti di Paolo Mantegazza». Archivio per l'antropologia e la etnologia, 56, 11-176.

Finocchi, L.; Gigli Marchetti, A. (a cura di) (2000). Editori e lettori. La produzione libraria in Italia nella prima metà del Novecento. Milano: Franco Angeli.

Frati, M.E. (a cura di) (1991). Le carte e la biblioteca di Paolo Mantegazza. Inventario e catalogo. Editrice Bibliografica: Milano.

Gazzetta, L. (2013). «Spiritualità, riforma educativa ed emancipazione femminile: una rete locale in età giolittiana». Chemello, A.; Finotti, F. (a cura di), Fogazzaro nel mondo. Vicenza: Accademica Olimpica, 511-31.

Käppeli, A.-M. (1990). Sublime croisade. Ethique et politique de féminisme Protestant, 1875-1928. Génève: Editions Zoè.

Mantegazza, P. (1876). La mia mamma. Laura Solera Mantegazza. Milano: Rechiedei.

Mantegazza, P. (1900). «A 16 anni sulle barricate di Milano». La vita italiana nel Risorgimento (1846-1849), vol. 3.II. Firenze: R. Bemporad \& Figlio, 5-42

Mantegazza, P. (1909). La Bibbia della Speranza. Torino: Società TipograficoEditrice Nazionale.

Marciano, A. (2004). Alfabeto ed educazione. I libri di testo nell'Italia post-risorgimentale. Milano: Franco Angeli.

Masiero, S. (2020). Amare, operare, sperare. Il contributo di Stéphanie Etzerodt Omboni alla società tra Otto e Novecento. Padova: Edizioni Diodati.

Minuto, E. (2017). «Un polic(t)eman? Il liberalismo umanitario di Gaetano Meale (1888-1900)». Aglietti, M.; Calabrò, C. (a cura di), Cittadinanze nella storia dello stato contemporaneo. Milano: Franco Angeli, 137-50. 
Pireddu, N. (2010). «Paolo Mantegazza: ritratto dell'antropologo come esteta». Chiarelli, C.; Pasini, W. (a cura di), Paolo Mantegazza e l'Evoluzionismo in Italia. Nuova edizione. Firenze: Firenze University Press, 187-203.

Ragone, G. (1999). Un secolo di libri: storia dell'editoria in Italia dall'Unità al postmoderno. Einaudi: Torino.

Reichenbach Pieczynska, E. (1902). Aux mères de famille. L'Ecole de la Pureté. Paris: Librairie Fischbacher.

Risso, R. (2020). «Gli ammonitori di Giovanni Cena: un romanzo sociale». Forum Italicum, 54(I), 297-311.

Sommario delle statistiche storiche dell'Italia (1861-1965) (1968). Roma: Istituto Poligrafico.

Strauss, D. (1876). L'antica e la nuova fede, traduzione autorizzata fatta sulla sesta edizione tedesca da S. O. E. Milano: Maisner.

Umano [Meale, G.] (1889). La fine delle guerre. Milano: Libreria editrice Galli.

Umano [Meale, G.] (1900). Patria Lex. il problema degli armamenti nazionali e delle guerre. Milano: Società editrice lombarda.

Vittoria, A. (2011). «Editoria e giornalismo». L'Unificazione. Roma: Istituto dell'Enciclopedia Italiana.

Vozza, V. (2017). «Fonti di storia dell'educazione a Padova: testimonianze documentarie dell'Istituto per l'infanzia abbandonata presso il Museo dell'Educazione dell'Università di Padova». Quaderni per la storia dell'Università di Padova, 50, 141-50.

Vozza, V. (2020). «Su alcune lettere di Stefania Omboni a Sibilla Aleramo (19011915). Donne moderne tra stampa emancipazionista, attivismo politico e aspirazioni profetiche». Quaderni eretici. Studi sul dissenso politico, religioso e letterario, 1(8), 59-124. https://doi.org/10.5281/zenodo.4569752. 



\title{
Quaderni Veneti
}

Vol. 9 - Dicembre 2020

\section{Rapporti culturali nella tradizione poetica del Novecento dalle lettere di Ernesto Calzavara}

Anna Rinaldin

Università Telematica Pegaso

\begin{abstract}
This article lists the best-known correspondents of Ernesto Calzavara, whose letters are kept in the Ernesto Calzavara Collection of the Archivio Carte del Contemporaneo, Ca' Foscari University of Venice. A complete list of the letters for each of them is provided, with a material description and some of the most exemplary passages. Some documents are also reproduced, as well as letters and some photos kept in the Collection.
\end{abstract}

Keywords Ernesto Calzavara. Archivio Carte del Contemporaneo. Correspondence. Dialectal poetry. Venetian dialect.

$\begin{array}{lll} & \text { Peer review } & \\ & \text { Submitted } & 2021-07-12 \\ \text { Edizioni } & \text { Accepted } & 2021-07-27 \\ \text { Ca'Foscari } & \text { Published } & 2022-02-17 \\ & \text { Open access } & \end{array}$

(c) 2022 | (@) Creative Commons Attribution 4.0 International Public License 
Nel Fondo Ernesto Calzavara, conservato presso l'Archivio Carte del Contemporaneo, ${ }^{1}$ è possibile scorrere i nomi di molti protagonisti della scena culturale del secondo Novecento italiano. Le lettere che compongono il ricco carteggio del poeta trevigiano sono testimoni di uno spaccato di vita letteraria di area specificamente veneta. ${ }^{2}$

In questo contributo, che intende integrare il Catalogo del Fondo Calzavara (cf. Rinaldin 2006), a cui si rimanda per un quadro d'insieme dei pezzi più significativi dell'intero Fondo, sono schedati criticamente alcuni dei più famosi interlocutori di Calzavara.

Le lettere insieme agli articoli costituiscono il materiale che traccia il percorso formativo di Calzavara, i suoi contatti, le fonti, le relazioni interpersonali. Critici, studiosi, giornalisti, poeti e scrittori compongono per Calzavara un comune fronte di crescita, di confronto e talvolta di scontro. Il carteggio nel suo insieme è consistente, visto che oltre alle lettere ricevute da Calzavara si conservano anche molte delle lettere mandate dal poeta stesso, sotto forma di minute.

Per la maggior parte si tratta di figure legate al mondo accademico: Calzavara cercò e mantenne sempre questo legame, con l'obiettivo di far passare la comprensione della propria opera poetica a livello istituzionale. Nella maggior parte dei casi, se si esclude qualche amico di infanzia come i coniugi Mazzolà o Giuseppe Scarpa (carteggi ricchi e personali), si tratta di professori universitari, giornalisti ed esperti del settore, a cui Calzavara inviava le proprie raccolte (del resto lo scrisse Calzavara stesso a Mario Chiesa nella lettera nr. 4).

Spiccano i carteggi con Vanni Scheiwiller, editore di molte delle raccolte poetiche, e Cesare Segre, che fu guida e riferimento fondamentale per il poeta. Col passare del tempo le relazioni e i riconoscimenti si arricchiscono, e fra gli anni Settanta e Ottanta compaiono i nomi di Stefano Agosti, Luciana Borsetto e Gianfranco Folena fra gli accademici, e di Biagio Marin, Giuseppe Pontiggia e Mario Soldati fra gli scrittori. Non mancano vari tentativi di passare a grandi

1 Ne è responsabile Silvana Tamiozzo Goldmann. L'Archivio è una delle anime principali del Centro Interuniversitario di Studi Veneti, afferente all'Università Ca' Foscari Venezia e diretto da Tiziano Zanato. Lettere e immagini del Fondo Calzavara qui riprodotte sono state donate all'Archivio dagli eredi del poeta.

2 Ne aveva già scritto Tamiozzo Goldmann (2006, 8-9). Il Fondo è attualmente in corso di riordino. Tuttavia il censimento sistematico dei corrispondenti (assieme alla descrizione degli interi fondi documentari di Ernesto Calzavara, Pier Maria Pasinetti, Armando Pizzinato, Carlo Della Corte, Bianca Tarozzi, Paolo Zolli) è già disponibile in http://sinapsi.copatitalia.com/sinapsi/. Per i testimoni delle poesie si farà riferimento al catalogo poetico completo curato da Tzortzis Ikonomou (Ernesto Calzavara. Immagini e poesia. Progetto di Digitalizzazione del Fondo Calzavara, prototipo disponibile in DVD in sede). Per le altre tipologie documentarie si darà una descrizione materiale in attesa del completamento della nuova inventariazione. Per una bio-bibliografia del poeta si rimanda al link https://www.unive.it/pag/fileadmin/user_upload/ dipartimenti/DSU/doc/Laboratori_Centri/FEBBR19_FondoErnestoCalzavara.pdf. 
case editrici, come Mondadori, cui Calzavara arrivò tramite Marco Forti e Mario Sansone.

Si può dire che ad ogni nome che compare nel carteggio - e sono tutti nomi di prestigio - sia legata almeno un'iniziativa che Calzavara riuscì a far concretizzare, magari con qualche insistenza. Per citare qualche episodio, rimandando alle singole schede per i dettagli, Mario Baratto presentò la poesia di Calzavara a Pordenone; Manlio Cortelazzo invitò Calzavara a preparare un intervento per il Corso di dialettologia veneta a Padova; Ettore Bonora organizzò un seminario sull'opera poetica di Calzavara. Il poeta Pablo Luis Ávila gli commissionò, insieme con Cesare Segre, alcune traduzioni poetiche in trevigiano per un volume dedicato a Jorge Guillén. E poi Carlo Della Corte fece conoscere Calzavara in RAI. Paolo Cherchi e Annalisa Cima chiesero e ottennero il dono di alcuni autografi per il Fondo manoscritti della Fondazione Schlesinger di Chicago. Con Giovanni Pozzi Calzavara scambiò notizie sul rapporto fra letteratura e immagini visive.

In questo modo il Fondo offre un'interessante mappa di relazioni, che rinvia a un fervente ambiente culturale cercato ansiosamente dal poeta, che in queste carte emerge in modo lucido. Calzavara vide la possibilità di rendere nota la sua poesia al pubblico, e ai suoi interlocutori chiese consigli redazionali ed editoriali. A molti spedì la propria opera: l'intento di diffondere ad ampio raggio i propri versi e il timore sempre palpabile che essi non fossero capiti e apprezzati sviluppò in lui un forte senso di autopromozione, in molti casi ripagato con parole di vivo apprezzamento.

Purtuttavia in questo contesto Calzavara raccontò poco della propria opera, e raramente vi espose le proprie riflessioni, come se aspettasse che fossero gli altri a prendere atto del valore della sua propria opera, senza indicazioni personali (quasi controcorrente in questo senso, si vedano le righe inviate a Ivano Paccagnella, nella lettera nr. 3).

Per ogni interlocutore, qui in ordine alfabetico, si forniscono l'elenco completo delle lettere ${ }^{3}$ e la trascrizione di alcuni dei passi più significativi. ${ }^{4}$ A corollario, è riprodotta una selezione di lettere e foto. ${ }^{5}$

3 Per la descrizione materiale si sono usate le seguenti abbreviazioni:

cart. ill. = cartolina illustrata

c. c. $=$ carta carbone

f. / ff. = foglio/i

lett. $=$ lettera

$\mathrm{mt} .=$ matita

telegr. $=$ telegramma cart. post. $=$ cartolina postale

datt. / datts. = dattiloscritto/i

fotoc. $=$ fotocopia

ms. / mss.= manoscritto/i

sf. = sfera (penna a)

vel. / vell. = velina/e

4 Segnalo che per collocare le singole poesie all'interno delle varie raccolte si può fare riferimento all'incipitario contenuto in Rinaldin 2006, 121-36.

5 Si tratta del nucleo principale dei materiali esposti alla Mostra documentaria contemporanea alla Giornata di studio su Ernesto Calzavara, tenutasi il 9 giugno 2006 pres- 


\section{Stefano Agosti}

Fra i primi a riconoscere il valore della poesia di Calzavara, nel gennaio 1975, Agosti presentò a Venezia la raccolta poetica di Calzavara Come se Infralogie: ${ }^{6}$ il fascicolo, inedito, formato di 22 fogli dattiloscritti e contenente la trascrizione da nastro della registrazione della giornata, è intitolato «Fondazione Querini Stampalia-Venezia. Presentazione del libro di poesie Come se. Infralogie di Ernesto Calzavara, di Stefano Agosti», evento che Calzavara ricordò spesso nelle sue lettere, anche inviando copia della trascrizione. Scrisse a Ottaviano Giannangeli il 13 gennaio 1976: «Il bravo Agosti presentò con molta penetrazione il mio Come se l'anno scorso (in sostituzione di Mario Baratto ammalato) alla Querini Stampalia di Venezia, dove intervenne molto pubblico qualificato». Il 16 dicembre del 1975 il critico scrisse per Il Giorno l'articolo «Come ridare vita e attualità a un linguaggio. Nei buchi del dialetto. Il caso singolare di Ernesto Calzavara che usa il trevigiano per ottenere risultati modernissimi di poesia. Pronomi, congiunzioni, particelle ricchi di significato». Ne scrisse un altro per lo stesso giornale il 5 marzo 1978, che però uscì mutilo e con un titolo redazionale fuorviante (si veda la lettera nr. 3); Agosti comunicò il disguido a Calzavara e inviò una copia del testo completo con il titolo originale «La linea "sintattica" dell'esperienza poetica contemporanea». Nel 1984 uscì la raccolta poetica calzavariana Le ave parole, ${ }^{7}$ di cui Agosti scrisse la prefazione («La lingua di Calzavara», 7-13), e che successivamente l'autore fece confluire in una propria raccolta di studi dal titolo Poesia italiana contemporanea. Saggi e interventi (Agosti 1995, 113-17).

1. E.C., Milano, 2 marzo 1976, lett. f. ms. sf.

«Prima che ci vediamo ti mando i tre saggi di traduzione in trevigiano [...] e 13 poesie mie - Elenco poesie per Agosti / Traduzioni: La salamandra, La mendacità della Radio, Dici sempre d'andar via ma non hai nemmeno la valigia / Poesie mie: Ombre sui veri, Quei conti, La porta tamburo, El menabò, Done sul tran, Omo del Nord Omo del Sud, Den, La corriera di Nax, A Tottenham Court, L'ingenuo computer, Bùtate drento, Ritorno, ${ }^{8}$ Pesi sul treno».

so l'Istituto Veneto di Scienze Lettere ed Arti, Venezia, i cui atti sono disponibili in Tamiozzo Goldmann 2007.

6 Calzavara 1974. Se ne veda, oltre che l'edizione, anche la storia del testo in Calzavara 2017a, 21-8.

7 Calzavara 1984. Se ne veda edizione e storia del testo in Calzavara 2017b, 7-33, 169-228.

8 La poesia «Ritorno» è conservata nel fondo in un unico testimone dattiloscritto, con coll. 8.42.2.4bis, ma resta inedita, quindi sarà esclusa da questo mannello che raccoglie alcune delle poesie di Analfabeto (Calzavara 1979; cf. Calzavara 2017b, 7-33, 37-168). 
2. E.C., Milano, 20 aprile 1977, lett. copia c. c. vel. ms.

3. S.A., Milano, 2 giugno 1978, lett. f. ms. sf. [fig. 1]

«Eccoti il testo integrale dell'articolo, apparso mutilo sul "Giorno" del 5-3-78 e col titolo redazionale La poesia c'è, ma il senso?, titolo che travisava interamente - come vedrai - il senso dell'articolo».

4. E.C., Milano, 27 febbraio 1979, lett. copia c. c. vel. datt.

5. E.C., 4 giugno 1979, lett. vel. ms. sf.

«Finalmente Analfabeto pubblicato. L'ho visto oggi da Guanda. Mi piace e credo che piacerà anche a te».

6. E.C., 21 dicembre 1979, lett. copia c. c. vel. ms.

«Oltre ai seminari propostimi per i prossimi mesi dalle Università di Padova e di Torino, ${ }^{9}$ spero che una rivista mi pubblichi qualcuna delle mie ultime composizioni».

7. E.C., Milano, 7 febbraio 1983, lett. copia c. c. vel. ms.

8. E.C., Milano, 7 maggio 1983, lett. copia c. c. 2 vell. datts.

«Ti mando fotocopie delle traduzioni in francese di poeti italiani in dialetto».

9. E.C., Milano, 18 ottobre 1983, lett. vel. ms. sf.

«Ti lascio Il cane e il cerchio. Vedrai anche tu se sarà il caso d'inserirla con le altre nella raccolta».10

10. E.C., Milano, 3 febbraio 1984, lett. copia c. c. vel. ms.

11. E.C., Treviso, 26 agosto 1984, lett. copia c. c. vel. ms.

«Ti accludo fotocopia della recensione di Porta [...]. Non sono molto d'accordo con lui in taluni punti per es. che il mio latino è folenghiano e macaronico, mentre è di uso e attuale. In ogni modo l'articolo è buono, con qualche spunto felice». ${ }^{11}$

9 Si vedano rispettivamente le schede su Gianfranco Folena ed Ettore Bonora.

10 Comparirà in Calzavara 1984.

11 Vedi Antonio Porta, lettera nr. 2. 


\section{Luciano Anceschi}

Nel 1984 Calzavara fece avere Le ave parole a Luciano Anceschi, fondatore nel 1956 de Il Verri. Sulle pagine della rivista, poco dopo l'uscita della raccolta nel dicembre del 1984, Anceschi ospitò la recensione intitolata «La poesia imperfetta» firmata da Niva Lorenzini (cf. Lorenzini 1984).

1. L.A., Rapallo, 28 aprile 1984, lett. 2 ff. mss. fotoc.

«Leggo - sto leggendo - con vivo interesse le tue poesie».

2. E.C., Milano, 7 maggio 1984, lett. copia c. c. vel. ms.

«Se arriverò ancora in tempo, vorrei pubblicare una antologia del meglio che ho fatto (bisognerà che cerchi l'Editore)».

3. E.C., Milano, 3 marzo 1985, lett. copia c. c. vel. ms.

«Il mio affetto e la mia stima in questa occasione trovano sinceri motivi di piacere».

4. L.A., Bologna, 23 marzo 1985, lett. f. ms. sf.

5. L.A., Bologna, 8 febbraio 1990, lett. f. ms. sf.

\section{Mario Baratto}

L'11 novembre 1977 Baratto presentò a Pordenone un incontro sulla poesia di Calzavara; il fascicolo contenente la trascrizione da nastro, formato da 32 fogli dattiloscritti, è intitolato «Biblioteca Civica di Pordenone. Mario Baratto. Presentazione dell'opera di Ernesto Calzavara». Poco dopo Calzavara gli manifestò le sue speranze per la valorizzazione artistica della cantata «Ritorno de Orfeo», composta l'anno prima dopo un viaggio a New York e che uscirà in Analfabeto (vedi Alfredo De Palchi, Willy Schwarz e Ludovico Zorzi).

1. M.B., Venezia, 3 febbraio 1976, lett. f. datt. [figg. 2-4]

2. E.C., Milano, 15 febbraio 1976, lett. copia c. c. vel. ms.

«In questi mesi [...] mi è saltato fuori qualche altro pezzo per la mia prossima nuova raccolta. Ma se penso che per pubblicarle bene dovrò affrontare forse moltiplicate le difficoltà e le amarezze che mi è costata l'uscita di Come se, mi viene lo sgomento».

3. E.C., Milano, 27 novembre 1977, lett. 2 ff. mss. fotoc. 
«Forse avrai letto il mio Orfeo. Chissà che quelli della Biennale me lo inseriscano in qualche modo nella manifestazione dell'anno venturo. Ripa di Meana mi disse che quanto a lui sarebbe d'accordo, ma poi sono anche gli altri a decidere».

4. M.B., Venezia, 4 dicembre 1977, lett. f. datt.

«Io credo che tu sia degno che si tenti, magari per mente e mano migliori delle mie, di tracciare una tua storia di poeta: anche la diacronia conta molto, in queste indagini, e non è affatto in contraddizione con un'analisi sincronica, se essa nasce al punto giusto e su un corpus individuato con lucidità nell'insieme dei materiali offerti da un autore. Ho letto, con interesse (in senso forte del termine) il tuo Orfeo: ma io, al tuo posto, non conterei molto sulla Biennale [...]. In altre parole, non so quando né in che modo ci occuperemo di nuovi programmi, né chi sarà il reggitore in questo campo. Puoi però contare fin d'ora, sul mio pieno e convinto appoggio: si tratta di cosa nuova, ardita sia nell'impasto linguistico che nella tematica proposta».

\section{Giorgio Bàrberi Squarotti}

Calzavara copiò o fece copiare due lettere ricevute dal critico, avute in risposta agli invii dei suoi versi; come spesso accade, le parole sono di vivo ringraziamento.

1. G.B.S., Torino, 6 marzo 1967, lett. copia c. c. vel. datt.

«La ringrazio vivamente del gentilissimo invio del Suo libro, ${ }^{12}$ che mi ha fatto molto piacere».

2. G.B.S., Torino, 24 marzo 1967, lett. copia c. c. vel. datt.

«Ricevo le Poesie dialettali ${ }^{13}$ e mi affretto a ringraziarLa vivamente anche di questo gentilissimo invio».

\section{Gian Luigi Beccaria}

La missiva di Beccaria si colloca fra le risposte, spesso molto benevole, di critici, scrittori e poeti ai quali Calzavara spediva la propria opera.

12 Probabilmente si tratta di Calzavara 1966, ora in Calzavara 2017a.

13 Calzavara 1960, ora in Calzavara 2006 e in Calzavara 2017a. 
1. G.L.B., Torino, 24 marzo 1984, lett. f. ms. fotoc.

«Che maestria ne Le ave parole, che bravura, quasi al limite, nel maneggio e nella riduzione della "lingua"! Mi ha fatto immenso piacere ricevere la sua raccolta. Altri i poeti dialettali che sino ad oggi conoscevo di più! Di suo io avevo sinora soltanto piluccato testi (non molti) e giudizi qua e là, molto in superficie; ora finalmente ho letto e riletto, silenziosamente e ad alta voce anche, una sua raccolta intera, godendomela tutta. La ringrazio davvero. Credo molto nella poesia "dialettale" (si fa per dire). Il pomeriggio dedicato, nelle tre giornate alessandrine, alla poesia dialettale è stato un successo autentico anche di pubblico (c'erano Baldini e Loi, tra gli altri). Ha visto le pagine bellissime (da un mio intervento d'apertura provocate) di Mengaldo sulla poesia dialettale, in "Sigma 2 "? Credo che Lei si riconoscerebbe molto in quello che Mengaldo scrive. Vivissimi complimenti dunque per i suoi versi».

\section{Adriano Bellotto}

Qualche mese prima della pubblicazione di Cembalo scrivano (Calzavara 1977) Calzavara si informava presso l'Archivio Storico Olivetti, ricorrendo al massimo esperto di cinema olivettiano, Adriano Bellotto, per ottenere le immagini del «più antico strumento di dattilografia», il cembalo scrivano dell'avvocato novarese Giuseppe Ravizza (1811-1885), ideale mezzo di riproduzione dei caratteri. I caratteri tipografici diventano protagonisti della poesia concreta del Cembalo. La ricerca delle origini si collega alla linea che appena due anni prima Calzavara aveva cominciato puntigliosamente a seguire nella ricerca delle più antiche, primitive forme alfabetiche, ricavando quella 'e' che, simbolo distintivo della sua poesia, decise di far scolpire su di un sasso posto nel giardino della sua casa di Treviso (vedi Antonio Zanussi). ${ }^{14}$ Bellotto indirizzò Calzavara al Museo Nazionale della Scienza e della Tecnica di Milano, che gli fece avere due foto, una delle quali sarà pubblicata in Cembalo scrivano [fig. 5].

1. E.C., Milano, 5 aprile 1977, lett. copia c. c. vel. datt.

«Nel mio libretto che dovrà pubblicare Scheiwiller (per iniziativa della Biblioteca Civica di Pordenone) di poesia concreta sperimentale in dialetto veneto, vorrei inserire l'unito capitoletto di dattilogrammi: Genesi con caratteri dattilografici appartenenti possibilmente al più antico strumento di dattilografia (Il cembalo scrivano 
di Ravizza) [...]. Una di queste, o altre foto di tipo longanesiano che fossero reperibili, potrebbe essere opportunamente riprodotta sulla copertina o nel testo».

2. A.B., Milano, 14 aprile 1977, lett. f. datt.

\section{Maria Teresa Biason}

Maria Teresa Biason fu fra le destinatarie de Le ave parole, che lesse e apprezzò.

1. B.M.T., Venezia, 20 giugno 1984, lett. f. ms. sf.

«Grazie per la sua arnia, per i suoi grafi, per quel nero su bianco di cui seguo con gioia l'apparire e il permanere».

2. E.C., Vetriolo Terme, 5 luglio 1984, lett. f. ms. sf.

«Sono contento che le mie api siano felicemente volate al suo giardino [...]. Voglio sperare che i lavori di Lei continuino a essere così apprezzabili e soprattutto così distinguibili come una delle opere sue che ho avuto recentemente per le mani».

\section{Giuseppe Biasuz}

Dal breve carteggio risulta la memoria di un professore di scuola di cui Calzavara serbava un sentito ricordo. Biasuz (1893-1991), di origini feltrine, fu saggista e scrittore, interessato ad argomenti legati alla cultura veneta.

1. B.G., Padova, 24 luglio 1978, lett. f. ms. sf.

«Ma veniamo subito al suo libretto di versi E Parole mate Parole pòvare [...]. L'ho subito letto di seguito e per intero, col piacere, lo dico subito, di ascoltare la voce e l'anima di un poeta nuovo».

2. E.C., Treviso, 21 agosto 1978, lett. copia c. c. vel. ms.

«Perché il mio libretto le è stato gradito e perché lo desidera, Le mando da Milano qualche altra pubblicazione».

3. E.C., Milano, 9 febbraio 1980, lett. copia c. c. vel. ms. 
«Lei una volta ci diede un tema d'italiano (Campane e sirene, o mi sbaglio?) che io invece di sviluppare in prosa, contrariamente certo alle sue intenzioni, svolsi in poesia. Lei ne fu sorpreso in quel momento ma poi alcuni versi le piacquero: Vidi sul campo quattro bovi bianchi / Traevan l'aratro curvi / Curvo su esso / il contadin premeva pieno di forze. Così invece di punirmi per la trasgressione, volle leggerli in classe e io ne fui molto soddisfatto. Vede professore, nella vita d'un uomo ci sono tanti anelli per la catena delle cause. Forse per me tra i tanti c'è stato anche il Suo».

4. B.G., Padova, 3 marzo 1980, lett. f. ms. sf.

«Grazie vivissime del ricordo, della lettera, del libretto Come se e della bella serata al Maldura».

5. E.C., Milano, 20 marzo 1980, lett. copia c. c. vel. ms.

«La ringrazio della sua affettuosa lettera piena di freschezza e di spirito».

6. B.G., Padova, 19 dicembre 1981, lett. f. ms. sf.

«Ricorda quando, a fine di anno, dicevo: Cari ragazzi, non vedo l'ora di non vedervi più. E voi: E si immagini noi, professore!».

\section{Giovanni Bonalumi}

Nel marzo 1990 Calzavara fu contattato dal prof. Renato Martinoni, che insieme con Bonalumi dirigeva a Basilea la rivista ticinese L'Almanacco. I due chiesero al poeta un inedito in dialetto da pubblicare sulla rivista. Calzavara inviò le inedite «Di una figura perduta»e «Reogio mato», pubblicate l'anno dopo nel nr. 10 della rivista, alle pp. 26-8; compariranno nel 1996 come poesie di apertura della sezione inedita di Rio terrà dei pensieri (Calzavara 1996). Bonalumi scrisse due articoli sulla poesia di Calzavara, che apparvero sul Corriere del Ticino e che Calzavara apprezzò molto: il primo uscito il 5 maggio 1990 e intitolato «Cosmogonia e vita quotidiana nell'opera di Ernesto Calzavara» (51), e il secondo «Un poeta nato due volte. Edito da Scheiwiller un volumetto di prose e versi dello scrittore Ernesto Calzavara: i sapidi divertimenti di un autore novantenne», (53), senza data ma certamente successivo all'invio di Rio terrà.

1. E.C., Milano, 22 febbraio 1990, lett. copia c. c. vel. ms.

2. G.B., Minusio, 7 marzo 1990, lett. f. ms. sf. [figg. 6-7] 
«La ringrazio dello splendido libro che subito ho saggiato nei suoi - come definirli? - gangli vitali. Uno straordinario libro!». ${ }^{15}$

3. E.C., Milano, 18 marzo 1990, lett. copia c. c. vel. ms.

4. E.C., Milano, 20 maggio 1990, lett. copia c. c. vel. ms.

«Ho molto apprezzato quello che Lei ha scritto specialmente per quanto riguarda la valutazione di quello che Lei chiama la mia filosofia».

5. E.C., Milano, 28 settembre 1990, lett. copia c. c. vel. ms.

«Come d'intesa, Le accludo in fotocopia le seguenti poesie: Inedite: I. Reogio mato (1988) in dialetto; II. Di una figura perduta in lingua».

\section{Ettore Bonessio di Terzet}

Calzavara conobbe Ettore Bonessio di Terzet in occasione dell'invito al convegno di poesia tenutosi fra New York e Genova nell'aprile del 1980, organizzato dal Centro Turati di Genova, per il quale Calzavara scrisse e presentò la poesia «La restera», che comparirà ne Le ave parole (Calzavara 1984). L'evento riscosse ampio interesse anche sulla stampa: il 27 aprile su Il Corriere della Sera comparve un articolo di Fernanda Pivano intitolato «Autori italiani e americani per un convegno a Palazzo Doria Spinola. Genova-New York, via poesia» (3) e uno su Il lavoro, il 26 aprile, «Grande interesse per l'incontro culturale di Palazzo Spinola. Genova e New York sono poeticamente gemelle. Si è concluso ieri il convegno internazionale Poesia e Interpretazione dedicato ai nuovi movimenti letterari italiani e statunitensi», di Roberto Amen. Poco dopo Bonessio di Terzet chiese a Calzavara alcuni consigli in merito alla pubblicazione di alcune sue opere che gli fece leggere e sulle quali Calzavara fornì precise indicazioni critiche ed editoriali, pur velate di un certo pessimismo.

1. E.C., Milano, 27 febbraio 1980, lett. copia c. c. vel. datt.

«Le mando una mia composizione (La restera) per la pubblicazione del Convegno insieme con una breve nota contenente i miei dati biografici e quelli delle mie raccolte di poesie».

2. B.T.E., Genova, 3 marzo 1980, lett. f. ms. sf.

3. B.T.E., Milano, 10 marzo 1980, telegr.

15 Si tratta di Calzavara 1990. 
4. B.T.E., Milano, 19 marzo 1980, telegr.

5. B.T.E., Genova, 19 marzo 1980, lett. 3 ff. datts.

6. B.T.E., Genova, 21 maggio 1980, lett. f. ms. sf.

7. E.C., Milano, 15 giugno 1980, lett. f. datt.

«Ho inseguito le parole della tua svelta poesia. Nel perimetro del tuo discorso che ricorda qualche volta il clinamen di certa area nordatlantica tra i dati reali e quelli culturali innervositi dalla fantasia, sai muoverti, mi pare, con humor, con leggerezza pensosa, con cronachismo divertito [...]. Tu mi chiedi della stampabilità poetica [...]. Credo che, sia pure dopo qualche riflessione e con qualche inevitabile taglio, tu possa tentare».

8. B.T.E., Genova, 1 luglio 1980, cart. post. ms. sf.

«Non negarmi qualche consiglio, perché ti stimo ragionevole e saggio oltre che poeta».

9. E.C., Vetriolo Terme, 9 luglio 1980, cart. ill. ms. sf.

«Come già ti dissi, di canali non ce n’è che uno: quello del potere culturale e sue ramificazioni, quali le case editrici spesso inaccessibili. Personalmente, io non ho alcun potere e di ciò feci esperienza attraverso lunghi anni di attese e di mortificazioni. Il tempo, la maturazione e l'insistenza paziente presso chi ha voce in capitolo e relativo potere, accontentandosi in principio di modeste edizioni, potranno farti raggiungere ciò che desideri».

10. B.T.E., Genova, 20 ottobre 1980, lett. f. datt.

11. E.C., Milano, 1 novembre 1980, lett. copia c. c. vel. datt.

12.E.C., Milano, 6 ottobre 1981, lett. f. ms. sf.

\section{Ettore Bonora}

Ettore Bonora scrisse un capitolo sulla poesia dialettale che compare nella Storia della letteratura italiana, dal titolo «Profilo della poesia dialettale. La poesia napoletana da Salvatore Di Giacomo a Eduardo de Filippo. Tessa, Giotti e altri» (Bonora 1976), in cui è citato anche Calzavara. Nella lettera del 20 dicembre 1979 Calzavara sottopose allo studioso l'esame delle poesie «Da Eraclito», «Studio n. 1», «Farmacia serada», «La Restera», «Finzion», «Studio n. 4», «Vis grata puellae», «Chiccus», «La ombra», e in quella del 13 gennaio «L'autorità del giudicato», «L'atto pubblico», «Perché tra uomo e uomo», «Teoria della presunzione», «L'assenza», «Ultrasuoni», «Incassato». Su questa scia, il 21 gennaio 1980 lo studioso organizzò presso l'Università 
di Torino un seminario sull'opera poetica di Calzavara. La trascrizione è conservata nel Fondo: il fascicolo di 3 fogli dattiloscritti è intitolato «Università di Torino. Facoltà di magistero. Seminario sull'opera poetica di Ernesto Calzavara col suo intervento e con la direzione di Ettore Bonora», che, insieme all'incontro del febbraio dello stesso anno organizzato a Padova da Folena (vedi Gianfranco Folena), sarà uno dei momenti maggiormente ricordati dal poeta. Nel 1981 Bonora scrisse l'articolo intitolato «Il dibattito sulla letteratura dialettale dall'età veristica ad oggi», pubblicato nel Giornale Storico della Letteratura Italiana, e nel 1984 sulla stessa rivista l'articolo «La condizione del poeta dialettale oggi», citando Calzavara in entrambe le sedi (cf. Bonora 1981; 1984). Nel settembre 1984 Bonora si complimentò con Calzavara per l'assegnazione del «Premio Dino Campana» (nel Fondo è conservata copia della «Motivazione del Premio Dino Campana ad Ernesto Calzavara (1984) letta da Aldo Rossi»).

1. E.C., 25 dicembre 1975, lett. f. ms sf.

2. E.B., Milano, 22 agosto 1978, lett. f. ms sf.

«Ho gradito molto le ultime poesie che mi ha dato modo di leggere. Pure non tentando vie imprevedibili, mi sembra che Lei non si accontenti dei risultati raggiunti e che non voglia ripetersi. La mia impressione è poi che sempre più nelle Sue ultime cose sia venuto castigando la vena idilliaca, rendendo più mordenti le Sue invenzioni. Circa i dattilogrammi del Cembalo scrivano [...] mi sembrano esercizi di wit [...] ma appunto per questo non capisco tutto, e non ne sono molto convinto».

3. E.C., Milano, 20 dicembre 1979, lett. vel. ms sf.

4. E.C., Milano, 13 gennaio 1980, lett. copia c. c. vel. datt.

5. E.C., Milano, novembre 1980, lett. vel. ms sf. e mt.

6. E.B., Milano, 8 giugno 1983, lett. f. ms sf.

«Non voglio perdere l'occasione di ringraziarti del pensiero di farmi avere anche le traduzioni delle tue poesie pubblicate in Euro$p e .{ }^{16}$ Sono contento che ti sia venuto un così apprezzabile riconoscimento».

7. E.B., Milano, 13 aprile 1984, lett. f. ms. fotoc.

8. E.B., Milano, 29 settembre 1984, lett. f. ms sf.

9. E.C., Milano, 5 ottobre 1984, lett. copia c. c. vel. ms.

16 Vedi Philippe Di Meo. 


\section{Luciana Borsetto}

Luciana Borsetto vanta una lunga conoscenza con il poeta e la sua opera, che segue - si può dire - passo passo. Nel 1979 scrisse l'articolo «La poesia di Ernesto Calzavara tra lingua e dialetto». Del 1980 è la recensione alla raccolta Analfabeto in Paragone (Borsetto 1979; 1980). Si citano almeno anche i saggi «Dialetto, antidialetto, "deriva". La scrittura poetica degli anni Settanta», «"Can-can, can-ton [...] canicole". Ernesto Calzavara tra lingua e dialetto», fino al più recente «Il "bestiario" di Ernesto. Per un ritorno alla poesia di Calzavara» (Borsetto 1984; 1989b; 2007).

1. L.B., Caselle, 28 maggio 1976, lett. 2 ff. datts.

«Sono una contrattista dell'Università di Padova e mi interesso di poesia dialettale del Novecento».

2. E.C., Milano, 2 giugno 1976, lett. copia c. c. vel. ms.

«Spero quindi in quel periodo di potermi incontrare con Lei per intrattenerci più comodamente e diffusamente sugli argomenti».

3. L.B., Caselle, 10 giugno 1976, lett. 2 ff. datts.

«La ringrazio moltissimo per la risposta così sollecita e così preziosa per la quantità e la qualità di materiale che mi ha reso disponibile».

4. L.B., Padova, 15 ottobre 1976, lett. f. datt.

5. E.C., Milano, 23 ottobre 1976, lett. copia c. c. 2 vell. mss.

6. E.C., Milano, 18 dicembre 1976, lett. copia c. c. vel. ms.

7. L.B., Padova, 13 dicembre 1976, lett. f. ms. sf.

8. L.B., Padova, 19 dicembre 1976, lett. f. datt.

9. E.C., Milano, 26 dicembre 1976, lett. copia c. c. vel. ms.

10. L.B., Padova, 4 gennaio 1977, lett. f. datt.

11. L.B., Padova, 2 maggio [1977], lett. f. ms. sf.

12.E.C., Milano, 11 maggio 1977, lett. copia c. c. 3 vell. mss.

«Ti mando qui unito, come desideravi, le fotocopie dell'articolo di Zanzotto per B. Marin».

13.E.C., Milano, 19 giugno 1977, lett. copia c. c. vel. ms.

14. L.B., Padova, 31 ottobre 1977, lett. f. datt.

15.E.C., Milano, 5 novembre 1977, lett. copia c. c. 2 vell. mss.

16. L.B., Padova, 18 novembre 1977, lett. f. datt.

«Sono costretta a chiederti un altro favore [...], cercare di sapere 
da Raboni (che tu mi dicesti conoscere bene) da dove si ricavano le citazioni di Giudici, Pasolini e Zanzotto».

17. E.C., Milano, 22 novembre 1977, lett. copia c. c. vel. datt.

18. L.B., Padova, 29 novembre 1977, lett. f. datt.

19. E.C., Milano, 18 dicembre 1977, lett. copia c. c. vel. datt.

20. E.C., Esino Lario, 31 dicembre 1977, lett. 4 ff. mss. fotoc.

21. L.B., Padova, 1 gennaio 1978, cart. ms. sf.

22.E.C., Milano, 9 aprile 1978, lett. copia c. c. 2 vell. mss.

«Torno adesso dal Circolo Turati dove in questi ultimi tre giorni ho assistito dal mattino alla sera allo svolgersi del seminario di studio sul tema Il movimento della poesia italiana negli anni '70».

23.L.B., Padova, 17 aprile 1978, lett. f. datt.

24. E.C., Milano, 24 aprile 1978, lett. copia c. c. vel. ms.

25.L.B., Padova, 27 maggio 1978, lett. f. datt.

26. E.C., Milano, 1 giugno 1978, lett. copia c. c. vel. ms.

27. E.C., Milano, 16 giugno 1978, lett. copia c. c. vel. ms.

28. L.B., Veli Losinj, 26 luglio 1978, lett. f. ms. sf.

29. E.C., Treviso, 20 agosto 1978, lett. copia c. c. vel. datt.

30. L.B., Roma, 26 ottobre 1978, lett. f. ms. sf.

31. E.C., Milano, 27 novembre 1978, lett. copia c. c. 2 vell. mss.

32.E.C., Milano, 9 dicembre 1978, lett. copia c. c. vel. ms.

33. L.B., Padova, 28 settembre 1979, lett. f. ms. sf.

34.E.C., 15 agosto 1979, lett. copia c. c. vel. ms.

35. L.B., Losino, 28 luglio 1979, lett. 3 ff. mss. sf.

36. L.B., Mali Losinj, 21 agosto 1979, lett. f. ms. sf.

37. L.B., Losino, 15 agosto 1979, lett. f. ms. sf.

38.E.C., Milano, 21 novembre 1979, lett. copia c. c. vel. ms.

«Ti ho telefonato tre volte in questi giorni [...] per dirti che Giovanni Raboni non avrebbe difficoltà a farti avere qualche sua lettera dichiarativa che vorresti».

39. E.C., Esino Lario, 28 dicembre 1979, lett. copia c. c. vel. ms.

40.E.C., Esino Lario, 2 gennaio 1980, lett. copia c. c. 3 vell. mss.

41. E.C., Esino Lario, 6 febbraio 1980, lett. copia c. c. 2 vell. mss.

42. E.C., Milano, 14 aprile 1980, lett. copia c. c. vel. ms.

43. L.B., Cagliari, 17 aprile 1980, f. ms. sf.

44.L.B., Padova, 11 dicembre 1980, f. ms. sf. mt.

45. L.B., Padova, 1 gennaio 1981, f. ms. sf.

46. E.C., Milano, 29 giugno 1981, lett. copia c. c. 2 vell. mss.

47. L.B., Padova, 1 gennaio 1982, f. ms. sf.

48. L.B., Veli Losinj, 10 agosto 1982, f. ms. sf.

49. E.C., Treviso, 18 agosto 1982, lett. copia c. c. vel. ms. 
«Ho cominciato in questi giorni la lettura del tuo studio sulle poetiche del ' $500 » .{ }^{17}$

50.E.C., Treviso, 21 agosto 1982, lett. copia c. c. vel. ms.

51. E.C., Treviso, 31 agosto 1982, lett. copia c. c. vel. ms.

52. L.B., Padova, 1 gennaio 1983, f. ms. sf.

53.E.C., Milano, 28 gennaio 1983, lett. copia c. c. vel. ms.

54.E.C., Milano, 18 febbraio 1983, lett. copia c. c. vel. ms.

55. E.C., Milano, 2 marzo 1983, lett. copia c. c. vel. ms.

56. L.B., Bressanone, 12 agosto 1983, 2 ff. mss. sf.

57. E.C., Cascina Valsassina, 22 agosto 1983, lett. copia c. c. vel. ms.

58. L.B., Torino, settembre 1983, f. ms. sf.

59. L.B., Padova, 1 gennaio 1984, f. ms. sf.

60.E.C., Milano, 2 aprile 1984, lett. copia c. c. 2 vell. mss.

61. L.B., Padova, 1 gennaio 1985, f. ms. sf.

62. L.B., Bressanone, 8 luglio 1985, 2 ff. mss. sf.

63. L.B., Padova, Pasqua 1985, f. ms. sf.

64. L.B., Padova, 15 gennaio 1986, lett. f. datt.

«Ti scrivo per ringraziarti del bellissimo dono natalizio, davvero inaspettato. Conoscevo Tessa attraverso i soliti prestiti della biblioteca. Ora entra nella mia pur modesta libreria».

65.E.C., Levico, luglio 1986, lett. copia c. c. f. ms.

66. L.B., Bressanone, 7 agosto 1986, f. ms. sf.

67. L.B., Veli Losinj, 20 agosto 1988, 2 ff. mss. sf.

«Nel corso dell'anno ho scritto anche un saggio sulla poesia di Giacomini. Non ho ancora pensato dove pubblicarlo, incerta se riunire tutti i miei lavori sui poeti in dialetto del Novecento in un volumetto». ${ }^{18}$

68.E.C., Treviso, 28 agosto 1988, lett. copia c. c. 2 ff. mss.

69. L.B., Padova, 1 gennaio 1989, f. ms. sf.

70.E.C., Milano, 9 agosto 1989, lett. copia c. c. f. ms.

71. E.C., Milano, 20 novembre 1989, lett. copia c. c. f. ms.

«A causa dell'incresciose vicende tipografiche editoriali non ti posso mandare ora che una delle copie [...] di Ombre sui veri con correzioni a penna». ${ }^{19}$

17 Si tratta probabilmente di Borsetto 1982.

18 Borsetto 1989c.

19 Si tratta della prima tiratura del volume del 1989, ritirato e ristampato l'anno dopo: Calzavara 1990. 
72. E.C., Milano, 1 maggio 1990, lett. copia c. c. vel. ms.

«Come tu desideravi ti mando qui unite le fotocopie di recensioni per Ombre sui veri in vari giornali».

73.E.C., Milano, 28 febbraio 1991, lett. copia c. c. f. ms.

74. L.B., Padova, 1 gennaio 1992, f. ms. sf.

75. E.C., Milano, 26 maggio 1992, lett. copia c. c. f. datt.

76. L.B., Padova, 1 gennaio 1993, f. ms. sf.

77. L.B., Padova, 23 dicembre 1994, f. ms. sf.

78. L.B., Padova, 24 agosto 1996, f. ms. sf.

\section{Thea Bozzi Dalla Cola}

Scrittrice di fiabe e racconti per ragazzi, Thea Bozzi è stata amica attenta e discreta di Ernesto Calzavara. Nel 1988, quando sembrava che le sue condizioni di salute stessero peggiorando, a lei raccomandò la pubblicazione della raccolta antologica il cui primo titolo Trilogia della ' $e$ ' ed altri versi venne in seguito sostituito con Ombre sui veri, e che nel 1990 lui stesso riuscì a portare a buon fine.

1. B.T., Moggio, 30 luglio 1978, lett. f. ms. sf.

«Questa volta, mio caro, ci sei: scrittore di alto e difficile sperimentalismo, ${ }^{20}$ come Zanzotto, tu, Ernesto Calzavara!».

2. E.C., Milano, 25 gennaio 1988, lett. copia c. c. 2 ff. mss.

«Nel caso che le mie condizioni di salute peggiorassero e non fosse più fisicamente possibile continuare a trattare e occuparmi con Scheiwiller, Garzanti e altri della pubblicazione antologica (Trilogia della e ed altri versi) che ora mi sta a cuore più di tutto, ti raccomando caldamente di occupartene tu».

\section{Franco Brevini}

Il rapporto con Brevini risale almeno alla data della prima lettera: l'8 dicembre 1979 la Radio della Svizzera Italiana mandò in onda, per la rubrica «Diario culturale», un'intervista del critico a Calzavara (nel

20 Si cita dall'articolo di Alberto Frasson dal titolo «Continua la nostra inchiesta sulle letterature regionali: Venezia e le altre province con uno sguardo al Trentino. Veneto, il dialetto come aristocrazia», in Tuttolibri, 4(29), 29 luglio 1978, 6-7. 
Fondo in 3 fogli dattiloscritti). Il 22 aprile 1990 sul Corriere della Sera apparve un articolo di Brevini sulla poesia in dialetto, dal titolo «Tutti gli specialisti da Pasolini in poi», in cui cita anche Calzavara. Lo stesso anno il critico pubblicò Le parole perdute, ${ }^{21}$ ampia e documentatissima raccolta di saggi sui dialettali del Novecento. Calzavara mostrò di non condividere alcune opinioni, e al riguardo scrisse un testo di risposta rimasto poi inedito (si veda Mario Chiesa, lettera nr. 32, a cui sono allegate le pagine).

1. E.C., 25 giugno 1979, lett. copia c. c. vel. datt.

2. E.C., 17 maggio 1991, lett. f. ms. sf.

«Ho letto recentemente il Suo nutrito volume Le parole perdute, $\mathrm{e}$ con particolare attenzione, quanto Lei ha scritto sulla mia opera. Mentre sono d'accordo con Lei per il suo riconoscimento del mio contributo al rinnovamento della poesia in dialetto, di ciò ringraziandola, [...] devo dichiararle invece che non sono d'accordo su alcuni punti che mi riguardano. Perciò ho ritenuto subito di chiarire a me stesso prima ancora che ad altri i motivi del mio dissenso e ho buttato giù alcune righe in proposito».

\section{Carlo Carena}

Nel marzo 1969 Calzavara chiedeva a Natale Mazzolà (vedi Natale Mazzolà, nrr. 9-10) qualche notizia sulle case editrici per una «eventuale pubblicazione». Mazzolà consigliò di contattare Mondadori e Pozza, interessate a pubblicare poesia, oltre che «i minori editori». Calzavara ribatteva che «c'è anche Einaudi che ha una nota collana di poesia moderna», e nell'ottobre di quell'anno a questa inviava le sue poesie. La risposta analitica ma essenziale arrivò per il tramite di Carena.

1. C.C., Torino, 21 maggio 1970, lett. f. datt.

«Abbiamo esaminate e discusse le Sue poesie a più riprese, facendole leggere a più colleghi come verifica di giudizio. L'impressione che si ricava da queste opinioni è di un indubbio interesse e di una poesia felicemente sostenuta nei suoi vari momenti. Ci pare però che, accanto a una grande cura e vigilanza formale, si avvertano ristagni di discorso e qualche compiacimento fine a se stesso, con ripetizioni di motivi e particolare insistenza su certe meditazioni e certi toni cantilenanti, che paiono a volte ricercati, voluti, a scapito della 
freschezza e dello scatto. La ricerca dovrebbe dunque svolgersi, a nostro parere, verso una maggior essenzialità e necessità, con un controllo più esigente della scrittura. Ma sono soprattutto dei motivi assai più concreti che ci costringono a rinunciare alla pubblicazione: e cioè il programma già fitto della Collana di poesia e lo spazio limitato, in essa, per i poeti italiani contemporanei, e dialettali».

\section{Paolo Cherchi}

Nel 1976 Calzavara donò alla biblioteca dell'Università di Chicago la raccolta Come se. Infralogie, ricevendo parole entusiastiche da Cherchi, che lì insegnava Letteratura italiana. Qualche anno dopo Cherchi propose a Calzavara di donare alcuni dei suoi autografi al fondo manoscritti della Fondazione Schlesinger di Chicago (vedi Annalisa Cima); Calzavara accettò di buon grado, allestendo appositamente una scelta di manoscritti.

1. E.C., Milano 3 giugno 1976, lett. copia c. c. vel. ms.

2. P.C., Chicago, 15 giugno 1976, lett. f. ms. sf.

«La ringrazio dell'omaggio alla nostra biblioteca e del grande piacere d'avermi concesso una lettura della Sua poesia: quanta intelligenza del mondo e quanto sentimento in quel gioco della disgregata infralogia! Un collega mi ha promesso che segnalerà il Suo libro su una rivista americana».

3. E.C., Milano, 18 dicembre 1976, lett. copia c. c. vel. ms.

4. P.C., Chicago, 5 luglio 1988, lett. 2 ff. datts.

«In veste di consulente del fondo autografi della Fondazione Schlesinger, e su suggerimento della dott. Annalisa Cima, presidente della stessa fondazione, mi permetto di scriverLe invitandoLa a considerare la possibilità di una donazione a questo fondo [...]. L'idea risale a Eugenio Montale, e il nucleo centrale del fondo è costituito proprio da autografi montaliani. A Eugenio Montale risale anche l'idea di dare a questo fondo una sede americana designata dalla Fondazione Schlesinger. La scelta è caduta sull'University of Chicago per varie ragioni, fra le quali basta menzionare la centralità della sede, il prestigio dell'istituzione, e soprattutto il fatto che nel dipartimento di manoscritti e rari siano depositati gli archivi della rivista Poetry, con autografi, quindi, dei maggiori poeti americani e inglesi del novecento. A questo si aggiunga la vasta raccolta di manoscritti legati all'università da molti dei suoi illustri docenti, da G.A. Borgese, a R. Stern, da E. Fermi a S. Bellow e M. Eliade [...]. L'italianistica americana di questi ultimi decenni è una disciplina in 
esplosione, e la letteratura contemporanea è oggetto di attenzione speciale. Sono convinto che una raccolta di autografi italiani verrà valorizzata al massimo. Il fondo accoglie autografi di poesia, di narrativa, di saggi a queste legati, carteggi, bozze e traduzioni. Data la natura della raccolta, gli autori invitati sono per lo più italiani e italo americani [...]. La nostra impresa è alle origini, ma sembra destinata solo a crescere. La Fondazione Schlesinger Le sarebbe infinitamente grata se volesse partecipare a questo processo di crescita con una donazione di autografi la cui scelta dipende soltanto da Lei».

5. E.C., Treviso, 18 agosto 1988, lett. copia c. c. vel. datt.

«Sono convinto della bontà della loro iniziativa che non mancherò di segnalare quando mi si presenterà l'occasione. Annalisa Cima me ne aveva parlato tempo addietro e per parte mia aderisco volentieri. Mi propongo di parlare ancora al più presto con l'amica comune dell'argomento per una prima scelta di autografi da inviare e, se gradito, altro materiale quale nastri, articoli, foto e filmati [...]. Intanto, nel ringraziarLa ancora dell'invito di cui mi dichiaro onorato, Le assicuro che Le sarò preciso più avanti».

6. E.C., f. ms. sf.

«Ho guardato tra le mie carte, ma di autografi veri e propri scritti a mano o a macchina in modo sistematico comprensibile delle mie raccolte, non ne possiedo».

7. P.C., Chicago, 14 dicembre 1988, f. ms. sf.

«Due righe per ringraziarLa di cuore della donazione al fondo autografi».

\section{Mario Chiesa}

Mario Chiesa curò con Giovanni Tesio (a cui si rimanda) il libro Il dialetto da lingua della realtà a lingua della poesia. Da Porta e Belli a Pasolini, ${ }^{22}$ molto apprezzato da Calzavara. Del 1982 è l'articolo «Appunti sullo studio della poesia contemporanea in dialetto» (Chiesa 1982). Nel 1984 ne uscì un altro, «Per una mappa della poesia contemporanea in dialetto» (Chiesa 1984). Nel 1984 Mario Chiesa e Giuseppe Tesio pubblicarono Le parole di legno, un'antologia in due volumi di poesia in dialetto del Novecento (Chiesa, Tesio 1984): Calzavara non solo è incluso nell'antologia 
con una selezione di poesie che i due autori tradussero in lingua con l'aiuto dell'autore, ${ }^{23}$ ma è anche l'ispiratore del titolo, tratto dal v. 10 della poesia «Ai materiali», compresa in Come se. Le lettere segnalate con asterisco sono state donate al Fondo da Mario Chiesa il 9 giugno 2006.

1. E.C., Milano, 14 aprile 1978, lett. copia c. c. 2 vell. datts.

2. E.C., Milano, 18 aprile 1978, lett. f. datt.*

«Il libro è veramente pregevole sotto molti aspetti; fra l'altro per la inquadratura generale, la penetrazione, la chiarezza, la ricchezza dei riferimenti e delle citazioni, la buona scelta dei testi riprodotti con le relative versioni e annotazioni per spiegare un discorso che è del tutto convincente».

3. M.C., Devesi di Ciriè, 21 aprile 1978, lett. f. datt.

«La ringrazio delle gentili espressioni di consenso per il lavoro mio e dell'amico Tesio. Tanto più che si vede chiaramente che non sono frasi d'occasione, ma opinioni frutto d'una attenta lettura».

4. E.C., Vetriolo Terme, 18 luglio 1979, lett. f. ms. sf.*

«Lei non può credere quanto sia di stimolo per il mio lavoro futuro il sentirmi capito fino alle mie ultime composizioni dagli addetti ai lavori».

5. M.C., Devesi di Ciriè, 25 gennaio 1980, lett. f. datt.

«Le tue poesie lette da te diventano comprensibilissime, non hanno veramente bisogno di commento [...]. Perciò se Segre [...] volesse farti un regalo (e farlo al pubblico) dovrebbe farti fare un audiolibro». ${ }^{24}$

6. E.C., Milano, 7 febbraio 1980, lett. f. ms. sf.*

«Quasi sempre nasce dentro di me la composizione come suono, nasce talora la pulsione-pensiero da dover scriverle prima ancora che me ne renda conto, che ne sia del tutto cosciente». ${ }^{25}$

23 Alla lettera nr. 17 sono accluse le traduzioni corrette dal poeta, in $4 \mathrm{ff}$. datts. Le poesie sono, in traduzione, «Parole matte», «Vengono da oltre quegli occhi», «Nel sonno», «La porta tamburo», «Il menabò», «Katacuna», «La casa», «I segni», «La catastrofe», «La risurrezione», «Le macchie di luce», «Numeri sempre Lucrezio», «L'Eterno».

24 Solo la riedizione di Ombre sui veri (Calzavara 2001) contiene un cd-rom con la lettura di Marco Paolini.

25 È nota l'importanza dei giochi fonetici nelle poesie di Calzavara, per cui cf. Calzavara $2017 \mathrm{a}, 15,24-5$. 
7. E.C., Milano, 23 febbraio 1980, lett. f. ms. sf.*

8. M.C., Devesi di Ciriè, 15 settembre 1980, lett. f. ms. sf.

9. M.C., Devesi di Ciriè, 18 settembre 1980, lett. f. ms. sf.

«Probabilmente prepareremo una antologia dei poeti in dialetto del Novecento in una collana di Einaudi per la scuola media».

10. E.C., Milano, 30 settembre 1980, lett. f. ms. sf.*

11. M.C., Devesi di Ciriè, 9 novembre 1980, lett. f. datt.

12.E.C., Milano, 13 novembre 1980, lett. f. ms. sf.*

13. M.C., Devesi di Ciriè, 19 dicembre 1980, lett. f. ms. sf.

14. E.C., Milano, 12 gennaio 1981, lett. f. ms. sf.*

15. M.C., Devesi di Ciriè, 25 aprile 1982, lett. f. ms. sf.

16. M.C., Ciriè, 11 gennaio 1984, lett. f. ms. sf.

«Ti mando copia della mia traduzione: manca una parola per $\mathrm{Ka}$ tacuna come vedrai; ma non sono molto tranquillo neppure del resto delle mie traduzioni».

17. E.C., 21 gennaio 1984, lett. f. ms. sf.*

«Ti accludo il tuo dattiloscritto di traduzioni delle mie poesie per la tua Antologia e fotocopie delle stesse con le mie correzioni. Si intende però, salvo per gli errori materiali, che tu ne terrai quel conto che credi».

18. M.C., Ciriè, 26 gennaio 1984, lett. f. ms. sf.

19. M.C., Ciriè, 22 marzo 1984, lett. f. ms. sf.

20. E.C., Milano, 2 aprile 1984, lett. f. ms. sf.*

«Ora devo occuparmi a fondo di formare una raccolta antologica del meglio che ho fatto finora. Ma le difficoltà editoriali mi sgomentano. Poi, una successiva raccoltina di scampoli in prosa (narrativa, la vecchia comunicazione all'Ateneo Veneto e idee sparse del mio taccuino notturno). E intanto il tempo stringe. Beati gli anni quando potevo dire che Il tempo non passa». ${ }^{26}$

21. E.C., Milano, 24 maggio 1984, lett. f. ms. sf.*

22.E.C., Vetriolo Terme, 4 luglio 1984, cart. ill. ms. sf.*

«Desidero ringraziare anche te come ho fatto con Tesio a voce per Le parole di legno con cui avete intitolato la vostra antologia [...].

26 Si tratta di Calzavara 1990 e 1996. L'ultimo è un riferimento alla prima plaquette del poeta, Calzavara 1946. 
Mi avete dimostrato la vostra stima, la vostra affettuosa amicizia e ve ne sono grato».

23. E.C., Milano, 20 gennaio 1985, lett. 3 ff. mss. sf.*

«Quando Pasolini lesse le mie composizioni nelle prime plaquettes, mi scrisse subito proponendomi di collaborare e promettendomi che mi avrebbe incluso nella nuova edizione della sua antologia». ${ }^{27}$

24. M.C., Ciriè, 24 gennaio 1985, lett. f. ms. sf.

25. M.C., Ciriè, 17 gennaio 1989, lett. f. ms. sf.

26. E.C., Milano, 28 gennaio 1989, lett. f. ms. sf.*

«L'influenza di certe poesie straniere che si va più diffondendo in Italia, porta arricchimenti notevoli e quasi imprevedibili alla poesia in dialetto».

27. E.C., Milano, 21 maggio 1990, lett. f. ms. sf.*

28. E.C., Milano, 22 maggio 1990, lett. copia c. c. vel. ms.

29. E.C., Treviso, 20 agosto 1990, cart. ill. ms. sf.*

30. E.C., Milano, 10 novembre 1990, lett. f. ms. sf.*

31. M.C., Ciriè, 14 marzo 1991, lett. f. ms. sf.

32.E.C., Milano, 27 marzo 1991, lett. f. ms. sf.*

«Sulla parzialità e gli atteggiamenti negativi di costui avevo cominciato a scrivere alcune cartelle da pubblicare (delle quali ti accludo fotocopia in via riservata) ma poi ho cambiato idea perché il discorso dovrebbe essere più lungo e per altri motivi del tutto diversi di cui ti parlerò a voce alla prima occasione». ${ }^{28}$

\section{Felice Chilanti}

Il 2 luglio 1969 Chilanti scrisse un articolo apparso sul Gazzettino di Venezia che fra titolo e sottotitolo recita «Viaggio tra i veneti sparsi in Italia. Gli intellettuali a Milano. Il poeta Calzavara rimane in volontario esilio per scrivere parole mate, parole pòvare. Adesso Comisso non c'è più ma Sozzati continua a dire: Treviso la nostra piccola Atene. Il necessario contatto con la poesia europea». Chilanti scrisse anche in seguito sulla poesia di Calzavara: il 12 giugno 1976 uscì

27 Le prime plaquettes del poeta risalgono tutte agli anni Quaranta. Si tratta probabilmente di Pasolini 1952; se ne fece una nuova edizione con prefazione di Giovanni Tesio (Pasolini 1995), ma Calzavara non compare neanche in questa.

28 Calzavara fa riferimento a Franco Brevini, alla cui scheda si rimanda. 
sulla Gazzetta del popolo «La poesia di Ernesto Calzavara. Il canto della ragione», di cui Calzavara fu entusiasta.

1. E.C., Milano, 15 dicembre 1975, lett. copia c. c. vel. ms.

«Mi fu poi gratissima la Sua assicurazione di aver già preparato un articoletto per il Corriere della Sera ${ }^{29}$ sul mio Come se che Le era piaciuto [...]. Pubblicare libri buoni va bene, ma se poi le voci migliori ne tacciono, viene lo sgomento».

2. E.C., Milano, 16 dicembre 1975, lett. copia c. c. vel. ms.

3. F.C., Roma, 4 aprile 1976, lett. 4 ff. datts e mss. fotoc.

4. E.C., Treviso, 23 agosto 1976, lett. copia c. c. vel. ms.

«Il Suo articolo mi ha dato soddisfazione perché ho sentito quella intensa comprensione che, anche per la comune conoscenza del nostro dialetto, Lei ha avuto della mia poesia e del sottofondo che me la fa lievitare. La ringrazio».

\section{Annalisa Cima}

Nel 1978 Annalisa Cima, insieme con Segre e Montale, diede vita alla Fondazione Schlesinger. ${ }^{30}$ Tramite Paolo Cherchi (a cui si rimanda) propose a Calzavara di donare alcuni autografi, e di pubblicare un volume di selezione di poesie (rimasto - questo - un proposito). Nel 1984 Calzavara scrisse la recensione del libro di poesie di Annalisa Cima Ipotesi d'amore (Cima 1984), da pubblicare in rivista dopo la supervisione di Cesare Segre.

1. E.C., Milano, 30 ottobre 1983, lett. copia c. c. 2 vell. mss.

2. E.C., Milano, 4 settembre 1984, lett. f. ms. fotoc.

«Eccoti il pezzo sul tuo libro che, come Thea ${ }^{31} \mathrm{mi}$ ha riferito ieri, tu desideravi. Non so se può andar tutto bene. In ogni modo prima di spedirlo per la pubblicazione a "L'occhio (o L'ozio?) letterario" o ad altra rivista o giornale come preferisci, sarà opportuno che

29 Ma uscirà sulla Gazzetta del popolo.

30 La Fondazione si costituì, in collaborazione con il Graduate Center di New York, nella seconda metà degli anni Settanta grazie al lascito della nonna austriaca della poetessa Annalisa Cima. Cf. Pivano, F., «Autori italiani e americani per un convegno a Palazzo Doria Spinola. Genova-New York, via poesia». Corriere della Sera, 27 aprile 1980, 3.

31 Thea Bozzi Dalla Cola, alla cui scheda si rimanda. 
tu lo rilegga e lo faccia rileggere al nostro bravo "Padre Cesare"32 per una verifica».

3. A.C., Lugano, 3 ottobre 1984, lett. f. ms. datt. fotoc.

«Nel prossimo anno [...] dovrebbe uscire il volume Selected poems by Ermesto Calzavara, California Press Fondazione». ${ }^{33}$

4. E.C., Milano, 27 maggio 1985, lett. copia c. c. f. datt.

5. E.C., Milano, 9 gennaio 1986, lett. copia c. c. vel. datt.

6. E.C., Milano, 3 febbraio 1986, lett. copia c. c. vel. datt.

7. E.C., Milano, 1 maggio 1990, lett. copia c. c. vel. ms.

\section{Pietro Cimatti}

Giornalista, caporedattore della Fiera Letteraria e presente nei programmi culturali della Radio, Cimatti fece uscire il 24 maggio del 1978 su Il Messaggero una segnalazione del settimo Almanacco dello Specchio (a cura di Marco Forti, a cui si rimanda) ${ }^{34}$ in cui compariva anche Calzavara (il titolo della recensione è «Il settimo Almanacco dello Specchio. Ricordate Scotellaro? C'è anche lui in questa vetrina disordinata»). ${ }^{35}$ Calzavara chiese spiegazioni sul giudizio espresso dal critico nei suoi confronti.

1. E.C., Milano, 19 giugno 1978, lett. copia c. c. vel. datt.

«Ho letto recentemente il Suo interessante articolo sul Messaggero del 25 Maggio u.s. Le sarei molto grato se volesse chiarirmi privatamente il significato della frase che mi riguarda: il falso

32 Il riferimento scherzoso è a Cesare Segre, a cui si rimanda.

33 Nel 1984 Calzavara appuntò su una velina: «Queste traduzioni in italiano dal dialetto dovranno servire allo scopo di facilitare la traduzione delle poesie in lingua straniera (eventualmente anglosassone)» (cf. la riproduzione della scheda in Rinaldin 2006, 92, dove si trova anche la riproduzione dell'autotraduzione in lingua di «Angelo», 24) Questa nuova attività iniziò proprio quando Annalisa Cima gli propose di curare la raccolta di poesie scelte "da tradurre in inglese e pubblicare in America | Novembre 1985» (è il fascicolo nr. 164 del Fondo, che porta sul frontespizio l'indicazione «Traduzione delle mie poesie in dialetto»). Si veda Calzavara 2017a, 32.

34 Segnalo anche la recensione di Cesare Viviani, uscita su Il Giorno l'11 giugno 1978: «L'Almanacco dello Specchio n. 7 a cura di Forti e Pontiggia. È di scena il corpo della poesia».

35 Calzavara 1978. Le poesie sono «Ombre sui veri», «La morte del giorno in paese», «La porta tamburo», «Croze fata col gesso», «Done sul tran», «El menabò», «Katacuna», «Le màcie de luce», «Disdoto respiri al minuto», «Iside», «Grafiti». Usciranno l'anno dopo in Analfabeto. 
dialettale zanzottiano Ernesto Calzavara. Voglio sperare che Lei mi accontenti. Ho il massimo rispetto delle opinioni altrui anche quando non mi sono gradite».

2. P.C., Roma, 30 giugno 1978, lett. f. datt.

«Perché dovrei non accontentarla? Per quello che l'Almanacco conteneva di suo, non ricordo da chi presentato, mi è parso come già detto che lei fosse un falso dialettale zanzottiano che è: un dialettale misto di non dialettale, con l'orecchio e la lingua ad altro, un po' indigeno e un po' metropolitano, un po' locale e un po' importato, un po' che sembra semplice e un po' che sembra complesso, o semplicemente colto. Questi miscugli di falso dialetto, magari per diventare un dialetto nuovo (ma parlato da una persona sola, l'autore) li ha fatti Zanzotto, e lei caro Calzavara li sa far bene. Tutto qui. Spero che anche questa estensione della mia opinione non le sia gradita: non amo essere gradito, preferisco giocare con i fiammiferi».

3. E.C., Milano-Treviso, 21 agosto 1978, lett. f. datt.

«Quanto poi a Zanzotto, come Lei sa, è appena da due o tre anni che scrive poesie in dialetto; io invece da più di trenta, evolvendomi in continuità. Quindi se colpa Lei ci vede, non è certo la prima quella del bravo Zanzotto ma la mia che l'ha preceduto di molto in tentativi di poesia diversa dalla tradizionale».

\section{Giuseppe Conte}

Calzavara mandò le sue opere allo scrittore Giuseppe Conte, che espresse in merito il suo compiacimento. Una delle raccolte poetiche di Conte, L'Oceano e il Ragazzo (1983), fu tradotta in francese da Jean-Baptiste Para, con cui Calzavara fu in contatto: per suo tramite, Philippe Di Meo (a cui si rimanda) tradusse alcune delle poesie di Calzavara.

1. G.C., Sanremo, 23 gennaio 1977, f. ms. sf.

«Non ho più dimenticato l'eloquenza rara, incantata, interrogante del suo Come se».

2. G.C., Sanremo, 14 maggio, 1978, f. ms. sf.

«Le devo queste due righe per dirle che ho trovato davvero fine e prezioso il suo Cembalo scrivano. Per non essere solo intenditore specifico di poesia visuale e concreta, ho trovato le sue tavole di 
esercizi molto fruibili e cariche di una forza di garbata semantizzazione che molte volte la poesia concreta e visiva non ha. Penso a Genesi, alla sua partitura vocalica».

3. E.C., Milano, 21 maggio 1978, lett. copia c. c. vel. ms.

«Le sono molto grato di quanto mi scrive e del suo particolare apprezzamento che mi conforta».

\section{Manlio Cortelazzo}

Lo studioso invitò Calzavara a presentare un intervento per il corso di dialettologia veneta, tenutosi a Padova nel giugno del 1980. Calzavara espose le tesi già sostenute dieci anni prima in occasione della comunicazione tenuta a Venezia presso l'Ateneo Veneto, dal titolo «Poesia in dialetto e poetiche moderne. Note» (Calzavara 1971), senza modificarne il contenuto. L'intervento è ristampato nel terzo volume della Guida ai dialetti veneti, curata dallo stesso Cortelazzo nel 1981 (Calzavara 1981).

1. M.C., Padova, 20 maggio 1974, lett. f. datt.

«Continuo ad apprezzare sinceramente la sua lirica e il Suo inedito atteggiamento verso un antico e rinnovato strumento linguistico».

2. M.C., Padova, 2 settembre 1980, lett. f. datt. fotoc.

«I partecipanti del Secondo Corso di dialettologia veneta (Padova, Giugno 1980) hanno espresso il desiderio di sentire dalla viva voce dei protagonisti il loro parere su che cosa significhi scrivere, oggi, poesia in dialetto».

3. E.C., Milano, 4 ottobre 1980, lett. f. datt. fotoc.

4. M.C., Padova, 13 ottobre 1980, lett. f. datt.

5. M.C., Padova, 25 marzo 1981, lett. f. datt.

6. E.C., Milano, 25 marzo 1981, lett. copia c. c. vel. datt.

7. E.C., Milano, 11 aprile 1981, f. ms. sf.

8. M.C., Padova, 20 maggio 1981, lett. f. datt.

\section{Maria Corti}

Calzavara inviò a Maria Corti una selezione di poesie per la pubblicazione in una delle sue riviste. 
1. E.C., Milano, 2 ottobre 1969, lett. copia c. c. vel. datt.

2. E.C., Milano, 16 dicembre 1975, lett. f. datt.

«Le mando le poesie per la rivista: un gruppo di composizioni, alcune delle quali in lingua, per il caso che quelle dialettali non fossero gradite. Tutte naturalmente inedite. Vedrà Lei, per favore, ben più esperta di me e conoscitrice dei gusti redazionali, quali scegliere e mandare. Ho aggiunto pure, nella eventualità che tutte o alcune di questo insieme, lasciassero dubbi, il gruppo scelto da C. Segre e da me per l'Almanacco dello Specchio ${ }^{36}$ e delle quali il direttore mi assicurò la pubblicazione non più, come pareva, per il '76, ma per il periodo successivo, dopo quelle di altri e nella speranza per tutti che non escano postume. Ombre sui veri, Galatea, L'arca, A Tottenham Court, Croze fata col gesso, Salvagnente, L'ingenuo computer, Done sul tran, Den, El menabò, Materiale, Quattro cieli, La morte del giorno, Aqua e piera, La porta tamburo, Pieni armaroni, La corriera di Nax, 'na man, L'imprevisto, Le macie de luce, Lamento de le do spose, Scarti semantici // Ombre sui veri, Galatea, L'arca, A Tottenham Court, Croze fata col gesso, Salvagnente, L'ingenuo computer, Done sul tran, Den, El menabò, Materiale, Quattro cieli, La morte del giorno, Aqua e piera, La porta tamburo, Pieni armaroni, La corriera di Nax, 'na man, Scarti semantici, Le ricordanze, Apuntamento».

3. E.C., Milano, 19 giugno 1978, lett. copia c. c. vel. datt.

\section{Robert Creeley}

Calzavara si informò presso Creeley circa una poesia di Charles Olson da lui edita, in merito alla congiunzione 'e'. Già titolo della raccolta poetica del 1966, è parola tematica nella produzione poetica di Calzavara, ${ }^{37}$ che decise di inciderla su una pietra del giardino della sua villa trevigiana. ${ }^{38}$

1. E.C., Milano, 10 giugno 1976, lett. copia c. c. vel. datt.

«Ho letto la poesia The Kingfisher di Charles Olson [...]. In questa poesia vi è, fra l'altro, un riferimento alla lettera E incisa in una vecchia pietra, che per ragioni particolari di carattere filosoficoletterario ha per me particolare interesse e molta importanza». 
2. R.C., Buffalo, 28 giugno 1976, lett. f. datt.

«It's my understanding that the $\mathrm{E}$ in question refers to an essay of Plutarch's, On the E On The Stone at Delphi - or a title similar to that. This was pointed out to me by Olson's German translator, Klaus Reichert [...], my presumption is that Reichert's identification is correct. But I never asked Olson directly myself [...]. I suggest you refer the question to George Butterick, Curator Charles Olson Archives».

\section{Gianfranco Crespan}

Crespan fu fondatore e presidente del Museo Etnografico Provinciale «Case Piavone» di Treviso. Nelle lettere Calzavara insistette sull'importanza della valorizzazione della cultura locale: uno dei progetti del poeta era l'istituzione di un «Archivio Storico Nazionale della Poesia italiana in dialetto», come annunciò nel filmato a cui fa riferimento Crespan, già nel 1985 (lettera nr. 2).

1. E.C., Milano, 14 ottobre 1980, lett. copia c. c. vel. ms.

«La vostra attività deve attirare l'attenzione [...] e promuovere nel pubblico quella consapevolezza della propria identità culturale che deve salvarsi dall'appiattimento in cui tra stampa Radio TV e pubblicità varie finisce col diventare sempre più di massa e perdere i migliori valori dell'individualità».

2. G.C., 15 novembre 1985, lett. f. ms. sf.

«Vorrei inviarle vivissime congratulazioni per il bel documentario che ho ammirato in televisione». ${ }^{39}$

3. G.C., Treviso, 2 aprile 1990, lett. f. ms. sf.

4. E.C., Milano, 11 ottobre 1993, lett. copia c. c. f. datt.

«La invito a [...] ritirare l'oca-girandola di ferro arrugginita, da me destinata al museo etnografico trevigiano che Lei dirige».

5. G.C., Treviso, 30 agosto 1995, lett. f. ms. sf.

39 Il filmato fu girato a Treviso nel marzo 1985, e trasmesso sul terzo canale dalla RAI il 22 ottobre dello stesso anno. Il testo si può oggi leggere integralmente in Rinaldin 2006, 95-103. 
«Ho letto un articolo sulla Tribuna di Treviso, a riguardo della sua poesia, e un raffronto con Andrea Zanzotto [...]. I lavori di restauro alle Case Piavone sono cominciati e speriamo che possano andare alacremente, cosicché il suo grande desiderio possa avverarsi, e che Treviso abbia in futuro il museo della civiltà contadina».

\section{Giorgio Cusatelli}

Il 28 aprile 1980 Cusatelli, germanista ed esperto di fiabe popolari e letteratura per ragazzi, pubblicò sul Corriere del Ticino l'articolo «Poesia senza premi», molto apprezzato da Calzavara.

1. E.C., Milano, 22 dicembre 1979, lett. copia c. c. vel. ms.

2. G.C., Milano, 11 febbraio 1980, lett. f. datt.

3. E.C., Milano, 14 marzo 1980, lett. copia c. c. vel. datt.

4. G.C., Milano, 26 maggio 1984, lett. f. datt. [fig. 8]

«Le porto molto ammirazione, dopo aver letto Le ave parole: aldilà dell'operazione plurilinguistica che Stefano Agosti ha decifrato con perizia, mi pare ne esca rafforzato lo scatto ideologico nella descrizione del rapporto con la mistificazione quotidiana (Finzion): è una paziente impazienza, un rodersi e divertirsi insieme, una scomposizion spinta talvolta sino ai grafismi, al segno ribelle».

5. E.C., Milano, 3 giugno 1984, lett. f. ms. sf.

\section{Manlio Dazzi}

Dazzi espresse più volte il suo apprezzamento per l'opera di Calzavara, che nel 1961 gli mandò alcune poesie inedite (lettera nr. 1).

1. E.C., Milano, 13 giugno 1961, lett. copia c. c. vel. datt.

«Aderendo al Suo invito gentile, Le mando questa breve raccolta di poesie che non inserii nel volumetto pubblicato l'anno scorso e che in parte non avevo ancora composto».

2. M.D., Asolo, 22 giugno 1961, lett. f. ms. sf.

«Ho letto le liriche che mi ha mandato. C'è la Sua voce».

3. E.C., Milano, 11 agosto 1962, lett. copia c. c. vel. datt.

4. M.D., Padova, 2 febbraio 1967, lett. copia c. c. vel. datt. 
«Freschissimo il tuo libretto, estroso, ma poeticamente, non intellettualmente, matto, ma umano, povero ma ricco di fantasia».40

\section{Libero De Libero}

De Libero inviò a Calzavara alcune considerazioni sulla fortuna editoriale della poesia.

1. L.D.L., Roma, 16 gennaio 1967, lett. copia c. c. vel. datt.

«Mala poesia di oggi va scritta e pubblicata senza attendersi alcunché, e penso che tu devi non far conto d'una realtà ormai ai limiti dell'assurdo».

\section{Carlo Della Corte}

Della Corte $^{41}$ fu una presenza costante nella vita di Calzavara. La prima intervista conservata nel Fondo è quella radiofonica trasmessa sul canale RAI di Venezia il 14 settembre 1974, in occasione della presentazione di Come se. Infralogie. ${ }^{42}$ L'11 settembre 1977 pubblicò un articolo dal titolo «Visita al poeta» comparso su Il Gazzettino (lettera nr. 2), nel 1978 un contributo nel volume Inchiesta sulla poesia. La poesia contemporanea nelle regioni d'Italia. Poeti nel Veneto (lettera nr. 3; cf. Della Corte 1979). Il 10 settembre 1979 mandò in onda per Radio Venezia un'intervista su Analfabeto, ${ }^{43}$ e il 18 giugno 1990 un contributo in merito a Ombre sui veri per la RAI, trasmessa poi anche a Radio Venezia (lettere nrr. 13 e 14).

1. C.D.C., Milano, 2 febbraio 1967, lett. copia c. c. vel. datt.

«La ringrazio di avermi dedicato il Suo splendido libretto di versi in dialetto». ${ }^{44}$

2. E.C., Treviso, 11 settembre 1977, lett. copia c. c. vel. ms.

40 Calzavara 1966

41 Come si è già detto in apertura, le «Carte del Contemporaneo» del Centro Interuniversitario di Studi Veneti accolgono anche il Fondo Carlo Della Corte. Si veda Gobbato, Uroda 2014, in part. 51-3, dove si fa riferimento alla stima e all'amicizia intercorsa tra Della Corte e Calzavara.

42 La trascrizione è proposta in Rinaldin 2020, 50-2.

43 Inedita, in Fondo Calzavara, Serie Prosa, fasc. 21, nr. 177.

44 Calzavara 1960 
«Contavo di farti a voce i rallegramenti per il tuo successo al Campiello, ${ }^{45}$ quando è venuta l'improvvisata sul Gazzettino di oggi».

3. E.C., Milano, 5 giugno 1979, lett. copia c. c. vel. ms.

«Solo ora ho potuto leggere quanto hai scritto su di me in Inchiesta sulla poesia per il Veneto. Sono contento del tuo giudizio molto preciso».

4. E.C., Milano, 15 ottobre 1979, lett. copia c. c. vel. ms.

«Eccoti [...] il libro di Steiner ${ }^{46}$ di cui ti avevo parlato, Teosofia».

5. E.C., 16 ottobre 1979, f. ms. sf.

6. C.D.C., Venezia, 1 novembre 1979, lett. f. ms. sf.

«Steiner è molto interessante, anche se mi lascia molte perplessità».

7. E.C., Milano, 19 settembre 1980, lett. copia c. c. vel. ms.

8. C.D.C., Venezia, 26 settembre 1980, lett. f. ms. sf.

9. E.C., Milano, 25 dicembre 1980, lett. copia c. c. vel. ms.

«Bravo. Un libro non comune. Non c'è soltanto la tecnica oramai consumata del romanziere, la rapidità, l'intensità vibratile moderna del tuo comporre». ${ }^{47}$

10. C.D.C., Venezia, 20 gennaio 1981, cart. ill. ms. sf.

«Grazie per la tua preziosa lettera, con tante e così acute indicazioni critiche, fin troppo benevole».

11. E.C., Milano, 7 dicembre 1985, lett. f. ms. fotoc.

12.C.D.C., Venezia, 18 febbraio 1990, lett. f. ms. sf.

«Ombre sui veri era il libro atteso da tutti e sono felice che sia uscito. Ne parlerò con solerte e appassionata adesione alla RAI».

13.C.D.C., Venezia, 2 luglio 1990, lett. 2 ff. fotoc. mss. sf.

14. E.C., Treviso, 16 agosto 1990, lett. copia c. c. vel. ms.

45 Della Corte ricevette il Premio Selezione Campiello con Cuor di padrone (Della Corte 1977).

46 Calzavara si rifà a Rudolph Steiner (anche esplicitamente) in molti testi poetici di Analfabeto, per cui si rimanda a Calzavara 2017b, passim.

47 Il riferimento è a Della Corte 1980. 
«Ho trovato a Milano la tua scheda culturale per la Radio, sulla mia antologia garzantiana».

\section{Alfredo De Palchi}

De Palchi, poeta di origini venete, trapiantato dal 1956 a New York, svolse un'intensa attività letteraria, destinata a diffondere la poesia italiana negli Stati Uniti. De Palchi e Calzavara si conobbero nel 1976, in occasione di un soggiorno di Calzavara a New York.

1. E.C., Levico-Vetriolo Terme, 16 luglio 1976, lett. copia c. c. vel. ms.

«Finalmente un poeta decente [...] fuori dalla tradizione e fuori dalle avanguardie, un indipendente [...]. Mi chiedo perché una poesia come la tua non sia conosciuta di più in questo nostro fetido paese».

2. A.D.P., New York, 15 novembre 1976, lett. 2 ff. mss.

«Ho letto le tue poesie subito dopo la tua partenza - sono bellissime, non solo come contenuto. Un linguaggio non solito d'un poeta che scrive in dialetto. Una tecnica (se così si può dire) non mortificata dalla mitezza e sdolcinatura che si riscontrano nella poesia dialettale in genere».

3. E.C., Milano, 18 dicembre 1976, lett. copia c. c. vel. ms.

«Il soggiorno stimolante di New York mi ha fatto inventare questa estate una cantata Ritorno de Orfeo del tutto speciale, da strutturare in musica (ma non ho ancora trovato il musicista). E il mito di Orfeo che risuscita a N.Y. in chiave moderna, assolutamente fuori da tutte le tradizioni letterarie». ${ }^{48}$

\section{Eugenio De Signoribus}

De Signoribus scrisse in merito alla rassegna di poesia dialettale comparsa sulla rivista Marka (numero monografico dal titolo «Poeti dialettali contemporanei», nrr. 6-7, novembre 1982-marzo 1983), dimostrando il proprio apprezzamento nei confronti della poesia di Calzavara, che vi compare con alcuni testi inediti.

48 Vedi Mario Baratto. 
1. E.C., Milano, 24 luglio 1982, lett. copia c. c. vel. datt.

2. E.D.S., Ascoli Piceno, 15 dicembre 1982, lett. f. datt. [fig. 9]

«Con sincerità e modestia, posso dirle che per me è uno dei più bravi. Quindi bisognerà cercare di comporre quello spazio in modo che presenti il meglio delle sue buone produzioni poetiche».

\section{Philippe Di Meo}

L'ampio carteggio con Di Meo, che merita una pubblicazione completa per la profondità della lettura testuale, è incentrato su questioni traduttive. Di Meo, traduttore in francese di molta letteratura italiana novecentesca (fra gli autori si ricordano Caproni, Gadda, Pasolini, Zanzotto), lavorò ad alcuni testi calzavariani ${ }^{49}$ in vista della pubblicazione in lingua francese sulla rivista Europe. Calzavara fu guida attenta e precisa, e glossatore di sé stesso. Il volume monografico della rivista uscì nel maggio 1983 (nr. 649) con il titolo Poètes dialectaux (Di Meo, Para 1983, 91-5).

1. E.C., Milano, 8 gennaio 1982, lett. f. datt. fotoc.

2. P.D.M., Parigi, 26 aprile 1982, lett. f. datt. fotoc.

«Preparando un'antologia di poesia italiana e ricordandomi della sua poesia intitolata Finzion pubblicata sul n. 67/68 di Nuovi Argomenti, vorrei tradurre e pubblicare alcune sue poesie in questa occasione».

3. P.D.M., Parigi, 9 maggio 1982, lett. 2 ff. datts. fotoc.

«Lei ha, tra molti pregi, quello di avere tolto la poesia in dialetto dalle secche del provincialismo arcadico astorico quanto la Sua lingua. In questo senso, la Sua è una poesia parificata con la poesia in lingua, anzi ritengo che Lei, con pochissimi altri, sia alla punta della ricerca poetica italiana in lingua o in dialetto che dir si voglia. Per di più integra felicemente il lato visivo (non soltanto iconico) come forse nessun altro [...]. Purtroppo non potrò tradurre più di 5 o 6 componimenti, per ragioni di spazio inerente ad ogni impresa antologica. Non è poco, giacché i due poeti che ne avranno 8 sono Luzi e Zanzotto».

4. P.D.M., Parigi, 5 dicembre 1982, lett. 2 ff. mss. fotoc.

49 Uscirono «Finzion» (da Calzavara 1980a), «Ombre sui veri», «Grafiti», «L'ingenuo computer», «La to presenza», «Nùmari sempre Lucrezio», «Den» (da Calzavara 1979). 
«Alcuni suoi componimenti mi han dato, e mi danno ancora, filo da torcere».

5. E.C., Milano, 11 dicembre 1982, lett. copia c. c. 5 vell. datts.

6. P.D.M., Parigi, 21 dicembre 1982, lett. f. ms. sf.

«La ringrazio sentitamente del suo indispensabile aiuto giacché il veneto lo afferro soltanto e senza di Lei non avrei fatto niente di buono».

7. E.C., Esino Lario, 31 dicembre 1982, lett. copia c. c. vel. ms.

«Salvo i punti da correggere sopraindicati, la traduzione adesso mi sembra che vada bene e, come già Le dissi, in certi punti ottima se non perfetta, per quello s'intende che è la mia conoscenza della lingua francese. Ho fiducia che essa sia ben giudicata dai competenti».

8. P.D.M., Parigi, 5 gennaio 1983, lett. f. ms. sf.

9. E.C., Treviso-Milano, 28 gennaio 1983, lett. copia c. c. vel. datt.

10. P.D.M., Parigi, 31 gennaio 1983, lett. f. ms. sf.

11.E.C., Milano, 16 febbraio 1983, lett. copia c. c. vel. datt.

12.E.C., Milano, 18 marzo 1983, lett. copia c. c. vel. datt.

13.P.D.M., Parigi, 30 marzo 1983, lett. f. datt.

«Sarei lieto di leggere e pubblicare le sue poesie francesi sulla mia rivista bilingue».

14.E.C., Milano, 19 aprile 1983, lett. copia c. c. vel. datt.

15. P.D.M., Parigi, 25 aprile 1983, lett. f. datt.

«C'è in questi versi una freschezza stupenda che non si potrebbe trovare nella poesia di un francese».

16. E.C., Milano, 16 maggio 1983, lett. copia c. c. 2 vell. datts.

«Circa il titolo della poesia $A n e a u^{50}$ di cui mi ha chiesto una spiegazione, l'avverto che esso non ha in se stesso un senso, ma si riferisce unicamente alle desinenze o finali delle parole della prima e seconda strofa che suonano rispettivamente an e eau [...]. L'opera è stampata bene, le scelte dei testi e le traduzioni sono ottime, come hanno riconosciuto anche i miei amici letterati».

50 In merito al titolo, si veda la lettera nr. 19 
17. P.D.M., Parigi, 19 maggio 1983, lett. f. ms. sf.

«La ringrazio di avere acconsentito a modificare le sue poesie».

18. E.C., Treviso, 4 agosto 1983, lett. copia c. c. vel. ms.

«Altre persone amiche hanno letto e trovato ottime le Sue traduzioni».

19. E.C., Milano, 30 gennaio 1984, lett. copia c. c. vel. ms.

«Ho rilevato che questo titolo ${ }^{51}$ non suona molto bene. Ne ho trovato un altro che la prego di sostituire al precedente, se arriverà ancora in tempo prima della pubblicazione. È il seguente Rides d'eau».

20. P.D.M., Parigi, 12 febbraio 1984, lett. f. ms. sf.

21. E.C., Milano, 24 febbraio 1984, lett. copia c. c. vel. ms.

22.P.D.M., Parigi, 15 aprile 1984, lett. 2 ff. mss. fotoc.

«La ringrazio di avermi fatto avere il suo bellissimo libro Le ave parole. L'uso che fa del latino è veramente mirabile».

23.P.D.M., Parigi, 5 maggio 1984, lett. f. ms. fotoc.

«In Lei la capacità di rinnovarsi è veramente sbalorditiva».

24. P.D.M., Parigi, 29 giugno 1984, lett. 2 ff. mss.

«Mi rallegro di vedere che la sua poesia si diffonda sempre più e interessi giustamente i massimi studiosi italiani».

25.E.C., Vetriolo Treme, 13 luglio 1984, lett. copia c. c. vel. ms.

26. E.C., Treviso, 26 agosto 1984, lett. copia c. c. vel. ms.

27. E.C., Milano, 4 ottobre 1984, lett. copia c. c. vel. ms.

«Le unisco Lentes Prières dopo averla un po' migliorata, tenendo conto anche delle sue particolari osservazioni e indicazioni tecniche».

28.P.D.M., Parigi, 19 ottobre 1984, lett. f. ms. sf.

«Sappia che l'interesse per la sua opera non è proporzionale alle (meritate e scontate) premiazioni di cui è l'oggetto anche se me

51 An eau, della lettera nr. 16. La poesia con questo nuovo titolo uscirà nello stesso anno per Le ave parole, e non sulle pagine della rivista. 
ne rallegro; la vera misura della validezza della sua operazione sta altrove».

29. P.D.M., Milano, 5 novembre 1984, lett. copia c. c. vel. datt.

30. P.D.M., Parigi, 8 novembre 1984, lett. f. ms. sf.

«Se Lei lo desiderasse, potrei pubblicare detto componimento ${ }^{52}$ in rivista, in Francia, ne varrebbe la pena!».

31. E.C., Milano, 3 dicembre 1984, lett. copia c. c. 3 vell. mss.

32.P.D.M., Parigi, 21 dicembre 1984, lett. f. ms. sf.

33. E.C., Milano, 21 gennaio 1985, lett. copia c. c. vel. ms.

«Le mie ricerche di poesia dialettale finora sono state poche e ancora non complete».

34.P.D.M., Parigi, 24 marzo 1985, lett. 2 ff. mss. fotoc.

«È Lei l'autore che ha più rinnovato la tradizione dialettale portandola al livello di ricerca di quella in lingua: da molto non capitava!».

35. E.C., Treviso, 16 agosto 1985, lett. copia c. c. vel. ms.

36. P.D.M., Parigi, 22 agosto 1985, lett. f. ms. sf.

37. P.D.M., Parigi, 18 dicembre 1985, lett. f. ms. sf.

«La ringrazio del suo interessante articolo sulla dialettalità nel quale sostiene l'unica giusta posizione. La pregherei di farmi di nuovo avere copia (fotocopia) del suo componimento intitolato $L a$ route des songes giacché lo voglio pubblicare sul primo numero di Vocativo». ${ }^{53}$

38.E.C., Milano, 27 dicembre 1985, lett. copia c. c. vel. datt.

«Le unisco la fotocopia da Lei richiestami della mia poesia (già dal titolo Lentes prières poi La route des songes [...]). Il 22 Ottobre u.s. dalla Radio Televisione Italiana, RAI (Sezione di Venezia) è stato trasmesso sulla rete veneta il mio filmato sulla mia poesia e sul mio ambiente originario che mi avevano invitato a fare a Treviso nella mia (ora solo in uso finché vivo, poi passerà a un Ente assistenziale e all'Università di Venezia Facoltà di Lettere) proprietà di Treviso dove nacqui: campi, giardino, vedute sulla città e paesi vicini.

52 «Lentes Prières» della lettera nr. 27, che uscì in Vocativo, 1, 1986, 170, e non comparve più nelle stampe, al posto di «La route des songes» di cui si dice alle lettere nrr. 37-38.

53 Si veda la nota alla lettera nr. 30. 
Il film della durata di mezz'ora "E.C. poeta della nostra terra", girato [...] in aprile, è riuscito abbastanza bene ed è stato apprezzato [...]. Mi piacerebbe che Lei un giorno lo potesse vedere e sentire». ${ }^{54}$

39. E.C., Milano, 3 giugno 1986, lett. copia c. c. 2 vell. datts.

«La rivista è una pubblicazione importante, di grande interesse, di notevole respiro per il livello dei testi originali e delle trattazioni critiche».

40. P.D.M., Parigi, 4 luglio 1986, lett. f. ms. sf.

«Se ci vorrà dare qualche poesia inedita, sarei ben lieto di pubblicarla e con tanto piacere».

41. E.C., Milano, 26 dicembre 1986, lett. copia c. c. vel. ms.

\section{Vico Faggi}

Vico Faggi, laureato in giurisprudenza e letterato come Calzavara, scrisse l'articolo «Sulla poesia di Calzavara», apparso il 22 maggio 1984 sul Giornale di Brescia.

1. E.C., Milano, 11 marzo 1980, lett. copia c. c. vel. datt.

2. V.F., Genova, 15 marzo 1980, lett. f. ms. sf.

«Ho ricevuto i preziosi volumetti e il materiale critico, molto utile».

3. E.C., Milano, 24 maggio 1980, lett. copia c. c. vel. ms.

«Così quando verrà il momento in cui potrò leggere il tuo testo scritto, sono sicuro che ne sarò altrettanto soddisfatto».

4. V.F., Genova, 28 maggio 1980, lett. f. ms. sf.

«Agli amici di qui vado raccontando che ti ho visto, e che ho imparato quanto sia importante e nuova la tua poesia».

5. V.F., Bologna, dicembre 1980, cart. ill. ms. sf.

6. V.F., Genova, 8 febbraio 1990, lett. f. ms. sf.

54 Vedi Mario Baratto. 


\section{Franco Fido}

Saggista e professore alla Harvard University, espresse il suo giudizio favorevole sulla poesia di Calzavara.

1. F.F., Cambridge, 6 marzo 1990, lett. f. ms. sf.

«Il collega e amico Dante Della Terza mi ha parlato con ammirazione del Suo recente libro di poesia veneta».

2. F.F., Rhode Island, 29 maggio 1990, f. ms. sf.

«Ho ricevuto la sua gradita lettera, e da Garzanti il volume Ombre sui veri: le migliori poesie in veneto da Noventa in qua, e degne di finire accanto ai migliori poeti viventi in italiano».

3. E.C., Milano, 2 aprile 1991, lett. copia c. c. f. datt.

4. F.F., Cambridge, 12 aprile 1991, lett. f. ms. sf.

\section{Armando Fiscon}

Nel 1972 Armando Fiscon istituì il «Premio Nazionale di poesia religiosa Camposampiero», a cui Calzavara partecipò con le poesie «Tra», «Vien da oltra quei oci», «Te si drento», «L'occhio di luce», «La gran volta». ${ }^{55}$ Calzavara risultò tra i primi dieci. Le poesie furono pubblicate nel volume miscellaneo Presenza religiosa nella poesia contemporanea italiana (cf. Fasolo, Fiscon, Rebellato 1974).

1. E.C., Milano, 20 aprile 1972, lett. copia c. c. vel. datt.

2. A.F., Camposampiero, 4 luglio 1972, lett. f. datt.

«Siamo lieti di comunicarLe che Lei è stato selezionato tra gli Autori che verranno inclusi nella Antologia della Poesia Religiosa italiana di cui al bando del presente I Concorso Nazionale di Poesia Religiosa».

3. E.C., Milano, 9 agosto 1972, lett. copia c. c. 2 vell. datts.

«Ho appreso con soddisfazione di essere stato prescelto tra i primi dieci classificati nel Concorso nazionale di Poesia Religiosa».

55 Le prime tre uscirono anche in Come se, la quarta era già uscita sulle pagine della rivista Il ponte, 11, nel novembre 1951, 1446, e l'ultima uscirà solo nel volume miscellaneo che si cita subito dopo. 
4. A.F., Camposampiero, 7 ottobre 1972, lett. f. datt.

«Ho l'onore di comunicarLe che Ella risulta incluso tra i dieci poeti vincitori del nostro Concorso Nazionale».

5. E.C., Milano, 6 novembre 1972, lett. copia c. c. vel. datt. [figg. 10-11]

«Innanzitutto desidero esprimerLe ancora il mio ringraziamento e la mia soddisfazione per il riconoscimento che mi è stato dato al Concorso».

\section{Gianfranco Folena}

Il 20 febbraio 1980 Calzavara fu al Circolo Filologico Linguistico Padovano su invito di Folena. La trascrizione da nastro è presente nel Fondo in cinque fogli dattiloscritti (FC, Serie Prosa, fasc. 9, nr. 97) ${ }^{56}$ Si tratta della presentazione della raccolta calzavariana Analfabeto. Di questo incontro scriverà a molti, e fra questi, l'anno dopo, il 9 dicembre 1981, all'amica Farida Galassi: «Una emozione quasi equivalente è stata per me l'anno scorso alla Facoltà di Lettere all'Università di Padova, dove il preside Folena, professore di fama, mi invitò a tenere un seminario e una lettura sulle mie ricerche per mie nuove poesie dialettali». Nello stesso 1980, un mese prima, Calzavara era stato invitato da Ettore Bonora (alla cui scheda si rimanda) all'Università di Torino, per un analogo seminario: i due eventi sono spesso ricordati come momenti importanti della propria esperienza poetica.

1. G.F., Padova, 18 gennaio 1980, lett. f. ms. sf.

«Vorremmo soprattutto far parlare Lei della Sua ricerca poetica e particolarmente linguistica e far parlare i testi che Lei potrebbe leggerci».

2. E.C., Milano, 30 gennaio 1980, lett. copia c. c. 2 vell. datts.

«La durata della mia esposizione sarà di 45 minuti poco più poco meno sulla scorta di appunti e un po' alla buona naturalmente, come già le dissi a Padova, da operatore di poesia e non da docente».

3. E.C., Padova, 1 marzo 1980, lett. copia c. c. vel. ms.

56 La trascrizione è proposta in Rinaldin 2020, 52-6. 
«Pensavo a Lei e sentivo come sento ora il desiderio di rinnovarle l'espressione del mio animo grato perché anche lei mi ha capito».

4. G.F., Padova, 16 ottobre 1987, cart. ms. sf.

«La lettera mi ha fatto molto piacere: trovare un cultore oggi è così difficile, e poi un cultore come te, non accademico».

5. G.F., Padova, 20 febbraio 1990, cart. ill. ms. sf.

«E un piacere nuovo e raro ritrovare tutte insieme le tue poesie in un volume». ${ }^{57}$

6. E.C., Milano, 4 marzo 1990, lett. copia c. c. vel. ms.

«Ti ringrazio molto delle tue parole così confortanti e amichevoli sulla mia antologia».

\section{Marco Forti}

Nel luglio 1969 Mario Sansone fece avere i versi di Calzavara a Marco Forti, per una possibile pubblicazione sull'Almanacco dello specchio edito da Mondadori. I versi non furono pubblicati, a causa della mancanza di spazio (Calzavara ne scrive sconsolato a Sansone e a Marin, a cui si rimanda). Nel 1975 si prospettò una nuova possibilità per l'Almanacco, il nr. 7, un volume monografico di poesia curato da Forti e Pontiggia. Il volume uscì tre anni dopo, nel giugno 1978, con versi inediti di Calzavara («Ombre sui veri», «La morte del giorno in paese», «La porta tamburo», «Croze fata col gesso», «Done sul tran», «El menabò», «Katacuna», «Le màcie de luce», «Disdoto respiri al minuto», «Iside», «Grafiti», uscite in seguito in Calzavara 1979). La sezione a lui dedicata ha il titolo «Ernesto Calzavara, Katacuna e altre poesie», con un'introduzione di Cesare Segre (159-80). Per la stessa rivista, nel nr. 10 del 1991, Calzavara pubblicò le poesie «Di una figura perduta», «Autonomia dell'oggetto», «Machina», «Reogio mato», «Qua ghe vorìa» (2-4, 26-9), di cui inedite «Di una figura perduta» e «Reogio mato», che usciranno in Rio terrà, e le altre già uscite in Analfabeto.

1. E.C., Badia, 15 luglio 1969, lett. copia c. c. vel. ms.

2. M.F. a Mario Sansone, Milano, 16 luglio 1969, lett. f. datt.

57 Calzavara 1990 
«Grazie del tuo ultimo biglietto relativo al nostro amico Calzavara. Puoi star certo che, per quanto riguarda me, farò tutto il possibile per i suoi versi. Il loro valore letterario è indubbio; purtroppo - non sembri assurdo - la letteratura e l'editoria non vanno sempre di pari passo: questo è lo scoglio da superare e che ancora non è stato superato. Volevo comunque assicurarti delle mie migliori intenzioni e del ricordo amichevole che ho della tua giusta raccomandazione per un poeta che merita».

3. M.F., Milano, 14 ottobre 1969, lett. 2 ff. mss.

«Col solo Specchio a disposizione [...], con più di due annate editoriali della collana praticamente già pronte, non possiamo dunque che restituirle i suoi libri, augurandole che presto un altro editore più agile di noi possa fare quella raccolta dei suoi versi».

4. E.C., Milano, 9 maggio 1975, lett. copia c. c. vel. datt.

«Le mando la raccolta delle mie poesie successive a Come se tutte inedite, tra le quali, Cesare Segre me ne ha scelte una decina che, secondo lui, potrebbero figurare nel prossimo Almanacco [...]. Scelta di poesie: Ombre sui veri, Galatea, L'Arca, A Tottenham Court, Croze fata col gesso, Salvagnente, L'ingenuo computer, Done sul tran, Den, El menabò».

5. M.F., Milano, 14 maggio 1975, lett. f. datt.

«Ricevo la sua del 9 maggio con accluso il manoscritto di versi nel quale, lei mi dice, Segre ha scelto quelle che potrebbero essere utilizzabili in Almanacco».

6. E.C., f. ms. sf.

7. E.C., Milano, 5 luglio 1975, lett. f. ms. sf.

8. E.C., 20 dicembre 1975, lett. f. ms. sf.

9. M.F., Segrate, 28 febbraio 1977, lett. f. datt.

«Lentamente, ma a seguito della tenacia sua e mia è venuto il momento di pubblicazione sull'Almanacco dello Specchio».

10. E.C., Milano, 21 marzo 1977, lett. copia c. c. vel. datt. 


\section{Maria Antonietta Grignani}

Nel 1985 Grignani scrisse la recensione di Le ave parole per Autografo (Grignani 1985). Nel 1990 espresse, all'uscita della raccolta antologica Ombre sui veri, un sincero apprezzamento.

1. E.C., Milano, 3 febbraio 1985, lett. copia c. c. vel. ms.

«Mi trovo quindi d’accordo sul Suo discorso critico e ammiro la sua ben provveduta ed elegante penetrazione del mio testo. Un'ottima recensione, veramente».

2. M.A.G., Pavia, 7 marzo 1990, lett. f. ms. fotoc.

«Ho tardato a ringraziarla per l'invio dello splendido volume che è un invito a festa per noi storici della lingua».

3. E.C., Milano, 18 marzo 1990, lett. copia c. c. vel. ms.

\section{Silvio Guarnieri}

Il critico comunicò a Calzavara il proprio apprezzamento.

1. S.G., Feltre, 11 febbraio 1990, lett. f. datt.

«Credo che d'ora in avanti chi parlerà di questa nostra poesia non potrà non tenerti presente».

2. E.C., f. ms. sf.

\section{Hermann Haller}

Haller fece uscire nel 1992 la recensione «Ernesto Calzavara. Ombre sui veri» sulla rivista L'anello che non tiene. Journal of Modern Italian Literature (Haller 1992). Calzavara ne apprezzò l'analisi, auspicando anche una pubblicazione in rivista italiana. Ma non sembra che il progetto abbia avuto seguito.

1. E.C., Milano, 6 maggio 1991, lett. copia c. c. f. datt.

2. H.H., New York, 30 maggio 1991, lett. f. datt. 
«Io terrò senz'altro conto del libro ${ }^{58}$ per un'eventuale nuova edizione del mio volume The Hidden Italy». ${ }^{59}$

3. H.H., New York, 25 aprile 1995, lett. f. ms. fotoc.

4. E.C., Milano, 17 maggio 1995, lett. f. datt.

«Insieme alla Sua del mese scorso, ho ricevuto L'anello che non tiene con la Sua recensione, una delle più persuasive, essenziali e penetranti che io abbia avuto. Perciò, sarei particolarmente felice se il Suo articolo fosse pubblicato anche in Italia. Le sono molto grato e vorrei scriverLe di più e meglio, ma sono diventato invalido e malato inguaribile. Spero di avere la fortuna d'incontrarLa un’altra volta».

\section{Vivian Lamarque}

Esprime a Calzavara le impressioni ricevute dalla lettura delle sue raccolte poetiche; il giudizio della poetessa è particolarmente apprezzato dal poeta.

1. V.L., Milano, 14 giugno 1980, lett. f. ms. sf.

«Le sue poesie sono state per me una scoperta molto bella [...]. Non mi chieda perché. Non so rispondere. Ma le dico che il suo libro mi ha commossa e che lo tengo tra quelli che mi sono più cari».

2. E.C., Milano, 30 giugno 1980, lett. copia c. c. vel. ms.

«Se le mie poesie Le sono piaciute, altrettanto mi è stata gradita la Sua lettera, ma di quel particolare gradimento che è proprio di chi anche da poche righe sente di essere stato capito nella sostanza».

3. V.L., Milano, 4 ottobre 1984, lett. f. ms. sf. [fig. 12]

«In libreria mi sono letta le tue preziose parole (me lo regalerò a Natale), sono proprio io la signora della macchina? Davvero? Che onore! Come ringraziarti?». ${ }^{60}$

58 Calzavara 1990

59 Haller 1986.

60 Si tratta forse della poesia «L'Auto-di-pioggia». 


\section{Alberto Limentani}

Lo studioso inviò a Calzavara una profonda analisi delle poesie contenute ne Le ave parole.

1. A.L., Padova, 29 aprile 1984, lett. f. ms. sf.

«Mi sembra di distinguere in queste sue creazioni due componenti [...]; una d'ordine formale-sperimentale, che si focalizza sui giochi sonori, le associazioni del significante, ecc, e che cerca prevalentemente per questa via di stringere nel dettato una realtà labile, che, in quanto ne resta nella morsa del linguaggio, si fa pregnante e creativa; l'altra di carattere evocativo, i valori perduti, la memoria trevigiana. [...] I risultati migliori sono dove la sintesi è completa, dal momento che l'una componente e l'altra rinviano alla carenza di vivibilità del mondo odierno e all'ancor più buio futuro. Dovessi suggerire io un testo, sarei per il beato Erico e La restera, da antologia».

2. E.C., Milano, 7 maggio 1984, lett. copia c. c. vel. ms.

«Le sono grato per l'attenta lettura del mio ultimo libretto di poesie. Mi è stato gradito quanto Lei mi ha scritto anche perché preciso, chiarificante».

\section{Niva Lorenzini}

Nel dicembre 1984 Niva Lorenzini recensì Le ave parole con un articolo intitolato La poesia imperfetta, uscito su Il Verri (Lorenzini 1984). Nel 1990 Calzavara chiese un'altra segnalazione sulla stessa rivista in occasione dell'uscita di Ombre sui veri.

1. E.C., Bologna, 5 marzo 1990, lett. f. ms. sf.

2. N.L., Bologna, 10 ottobre 1990, lett. f. ms. sf.

«È un libro importante e l'ho inserito, per ora, in una rassegna di poesia contemporanea che uscirà nel marzo '91 su una rivista del Mulino (L'informazione bibliografica)».

3. E.C., Milano, 10 novembre 1990, lett. copia c. c. vel. ms. 


\section{Biagio Marin}

Marin fu uno dei primi a scrivere sulla poesia di Calzavara: nel 1967 uscì su Il Gazzettino l'articolo «Un poeta dialettale. La e di Calzavara. Un linguaggio trevisano limpido e trasparente di una melodiosità che trascina come una pagina di Vivaldi». Nel 1967 Calzavara espresse al poeta gradense la delusione per la restituzione delle proprie poesie da parte di Mondadori, in quanto Marco Forti si era dichiarato impossibilitato a pubblicarle (vedi Marco Forti). Anche Marin raccontò a Ernesto le proprie vicende editoriali.

1. E.C., Milano, 18 dicembre 1969, lett. copia c. c. vel. ms.

«Ho fatto qualche cosa, forse non tutta da buttare; ma che vale, se dopo non puoi comunicare, se cioè è divenuto estremamente difficile veder pubblicate e diffuse le proprie opere?». ${ }^{61}$

2. B.M., Grado, 25 dicembre 1969, cart. post. ms. sf. [fig. 13]

«Alla fine di giugno uscirà a Trieste un volume unico con tutta la mia produzione di versi dialettali dal ' 12 al $70 .{ }^{62}$ Io di qui non $\mathrm{mi}$ muovo più».

3. E.C., Milano, 19 ottobre 1979, lett. copia c. c. vel. ms.

«Mi affretto a congratularmi con te per il doveroso omaggio che ti ha reso la tua terra. Tu ne sei lo spirito più vibrante e alto [...]. Il tuo Assoluto, il tuo Eterno sono anche i miei. La tua poesia e per quel passo anche la mia, tendono ad essi, cioè alla vera Essenza».

4. B.M., Grado, 30 ottobre 1980, lett. f. datt.

«Sono in attesa dell'uscita di un libretto di versi miei della Bur; ${ }^{63} \mathrm{e}$ forse nel prossimo anno la Garzanti pubblicherà un'antologia che verrà curata da Claudio Magris. ${ }^{64}$ Saranno i documenti del mio congedo dalla vita».

61 Calzavara fa riferimento al primo rifiuto di pubblicazione per l'Almanacco dello Specchio, per cui vedi Marco Forti.

62 Nel 1970 uscirono sia I canti dell'isola. 1912-1969 (Marin 1970a, a cui fa riferimento Marin nella lettera) sia La vita xe fiama. Poesie 1963-1969 (Marin 1970b).

63 Marin 1981a.

64 Marin 1981b. 


\section{Maria Calzavara Mazzolà}

Moglie di Natale Mazzolà (alla cui scheda si rimanda), Maria Calzavara (che non aveva rapporti di parentela con Ernesto) tenne un carteggio antico e molto fitto con Ernesto, meritevole di pubblicazione integrale. I tre si conobbero prima della partenza di Ernesto alla volta di Milano, nei primi anni Trenta. A Treviso Calzavara lasciò la sua stretta cerchia di amici, formata, oltre che dai due coniugi, anche da Giovanni Comisso, Gino Scarpa e Ciro Cristofoletti. Maria ed Ernesto adottarono un nome di penna, lei Gaia e lui Felton (e Natale Gaio). Lo scambio epistolare presente nel fondo risale alla fine degli anni Cinquanta, e consiste per lo più in informazioni letterarie: Maria lesse le poesie di Calzavara, annotando le proprie considerazioni sulle copie che Calzavara via via le mandava. Calzavara le vagliava attentamente e decideva se modificare o meno le proprie poesie. Dal canto suo Maria teneva al corrente Ernesto sulle pubblicazioni che curava col marito, e fra queste l'edizione del carteggio con Giovanni Comisso, ${ }^{65}$ di cui Maria non vedrà la stampa (morirà nel 1970). Il 9 dicembre 1959 Maria Mazzolà chiese il permesso a Calzavara di pubblicare parte di una lettera da lui inviata da Milano in un testo di memorie di guerra, Cronache partigiane. ${ }^{66}$

1. M.C.M., Roma, 9 dicembre 1959, lett. f. datt.

«Vi è un accenno di tre righe al bombardamento aereo su Treviso del 7 aprile 1944. Noi le chiediamo di poter mettere in nota - in quanto per economia di materia non possiamo allungarci nel testo - parte della lettera che lei mi scrisse il 16 da Milano sotto l'impressione immediata di quanto aveva visto a Treviso. È bellissima, tragica e poetica».

2. E.C., Milano, 11 dicembre 1959, lett. copia c. c. vel. datt.

«Sento con piacere che Gaio sta preparando per le stampe i Suoi ricordi di partigiano [...]. Quanto alla mia vecchia lettera sul bombardamento di Treviso, non credevo che l'aveste conservata e che meritasse l'onore di essere pubblicata sia pure in nota. Vi ringrazio».

3. M.C.M., Roma, 25 dicembre 1959, lett. f. ms. sf.

4. E.C., Milano, 5 gennaio 1960, lett. copia c. c. vel. datt.

«Ho avuto la Sua di Natale e la copia della mia del 16.4.44 alla quale, sempre che siate d'accordo anche voi, già che l'avete in qual- 
che punto giustamente corretta, direi di apportare le seguenti piccole modifiche».

5. E.C., Milano, 12 maggio 1964, lett. copia c. c. vel. datt.

6. M.C.M., Roma, 16 maggio 1964, lett. f. datt. e ms. sf.

«Inviandole il libro pensavo vagamente che lei, sempre tanto gentile, mi avrebbe forse scritto una bella lettera. Ma tanto bella non la immaginavo».

7. E.C., Milano, 24 febbraio 1965, lett. copia c. c. vel. datt.

«Se, a matita, mi indicherete con un segno quelle [poesie] che vi piacciono, quelle da scartare (e non saranno poche) ed eventualmente qualche breve nota marginale, senza però farvene obbligo, ve ne sarò molto grato».

8. E.C., Milano, 11 aprile 1965, lett. copia c. c. vel. ms.

9. E.C., Milano, 10 ottobre 1965, lett. copia c. c. vel. ms.

10. E.C., Milano, 23 ottobre 1965, lett. copia c. c. vel. ms.

11. M.C.M., Roma, 27 ottobre 1965, lett. f. ms. sf.

12.E.C., Milano, 10 dicembre 1965, lett. copia c. c. vel. ms.

«Ho già mandato a Scheiwiller figlio già dai primi di novembre [...]. D’altra parte oggi a stampar versi c'è quasi da vergognarsi».

13. M.C.M., Roma, 15 dicembre 1965, lett. f. ms. sf.

14. E.C., Milano, gennaio 1966, lett. copia c. c. 2 vell. mss.

15. M.C.M., Roma, 6 aprile 1966, lett. 2 ff. datts.

«Il Censore ${ }^{67}$ poi trema sulle due nuove poesie da inserire. Se sono porcherie vorrebbe vederle per prendersi il gusto di strapazzarla a squarciagola. Non avrà dimenticato, spero, gli urli della scorsa estate al Circeo».

16. E.C., Milano, 18 maggio 1966, lett. copia c. c. 2 vell. datts.

«La Censura vorrebbe dunque leggere le nuove poesie? Lo temevo e lo desideravo. Eccole qui unite».

17. E.C., Milano, 9 agosto 1966, lett. copia c. c. vel. datt.

18. M.C.M., San Felice Circeo, 15 agosto 1966, lett. 2 ff. datts.

67 Maria si riferisce scherzosamente al marito. 
«È un libretto nutrito, per niente monotono (una poesia tira l'altra); soffuso anche di un quid (poetico) che lascia l'eco nell'animo del lettore a libro chiuso [...]. Le dirò ora per ultimo che le sue nuove poesie mi sono piaciute; le nucleari, dovrei dire la nucleare, coglie poi un argomento nuovissimo, senza disumanizzarlo».

19. E.C., Milano, 3 settembre 1966, lett. copia c. c. vel. datt.

20. E.C., Milano, 4 ottobre 1966, lett. f. ms. sf.

21. E.C., Milano, 18 dicembre 1966, lett. copia c. c. vel. ms.

«Finalmente l'altro giorno, con alquanto ritardo [...], il libretto è stato stampato e rifinito. Nelle librerie viene distribuito tra un mese». ${ }^{68}$

22. M.C.M., Roma, 19 dicembre 1966, lett. f. ms. sf.

23.E.C., Milano, 13 febbraio 1967, lett. copia c. c. 4 vell. mss.

24. M.C.M., Roma, 13 marzo 1967, lett. 2 ff. datts.

25.E.C., Milano, 19 marzo 1967, lett. copia c. c. vel. ms.

26. M.C.M., Roma, 23 marzo 1967, lett. f. ms. sf.

27. E.C., Milano, 10 aprile 1967, lett. copia c. c. vel. ms.

28.E.C., Milano, 16 aprile 1967, lett. copia c. c. vel. ms.

29. M.C.M., Roma, 20 maggio 1967, lett. f. datt. e ms. sf.

30. E.C., Treviso, 31 maggio 1967, lett. copia c. c. vel. ms.

31. E.C., Milano, 4 ottobre 1967, lett. copia c. c. 3 vell. mss.

«Ho qui davanti le vostre lettere di A. Martini, di cui ho letto e vado leggendo e rileggendo pagine meravigliose e struggenti. Non è un libro comune». ${ }^{69}$

32.E.C., Milano, 23 ottobre 1967, lett. copia c. c. 3 vell. mss.

33. M.C.M., Roma, 7 dicembre 1967, lett. f. ms. sf.

34.E.C., Milano, 12 dicembre 1967, lett. copia c. c. 2 vell. mss.

35. M.C.M., Roma, 8 gennaio 1968, lett. f. ms. sf.

«Concludendo ho passato con le sue poesie due ore bellissime e la ringrazio di avermele mandate in anticipo».

36. E.C., Milano, 21 gennaio 1968, lett. copia c. c. 4 vell. mss.

37. M.C.M., Roma, 26 gennaio 1968, lett. 2 ff. mss. sf.

38. M.C.M., Roma, 29 gennaio 1968, lett. 2 ff. datts.

39. M.C.M., Roma, 5 febbraio 1968, lett. f. datt.

68 Calzavara 1966.

69 Mazzolà, Calzavara Mazzolà 1968. 
«Non si dia pena di rispondere ai miei pensamenti, non perda tempo. Glieli ho mandati perché le voglio bene e ogni tanto temo che lei svii e traligni in fumisterie, problemi inutili e sbagliati che fa lo stesso, con grave danno della sua personale vena poetica».

40. M.C.M., Roma, 28 febbraio 1968, lett. f. ms. sf.

41. M.C.M., Roma, 28 marzo 1968, lett. f. ms. sf.

42. M.C.M., Roma, 3 aprile 1968, lett. f. datt.

43. M.C.M., Roma, 5 aprile 1968, lett. f. datt.

44. M.C.M., Roma, 6 aprile 1968, lett. 2 ff. datts.

45. M.C.M., Roma, 7 aprile 1968, lett. f. datt.

46. E.C., Milano, 9 aprile 1968, lett. copia c. c. 3 vell. mss.

47. E.C., Milano, 11 aprile 1968, lett. copia c. c. 2 vell. datts.

«Eccole tre varianti di Quel sabo de Setembre». ${ }^{70}$

48. M.C.M., Roma, 17 aprile 1968, lett. f. datt.

49. E.C., Milano, 19 aprile 1968, lett. copia c. c. 2 vell. datts.

50. E.C., Milano, 9 maggio 1968, lett. copia c. c. vel. datt.

51. M.C.M., Roma, 14 maggio 1968, lett. f. datt.

52. E.C., Milano, 16 maggio 1968, lett. copia c. c. vel. ms.

53. M.C.M., Roma, 4 giugno 1968, lett. f. datt.

«Quel cancro a metà del Tennis ${ }^{71} \mathrm{mi}$ ha tolto il respiro e rialzato la pressione a 180 (sono in cura). Ho finito la poesia di volata e pensato che non l'avrei più riletta. Invece, dopo un'ora ci sono tornata sopra. Quel campo di tennis è una meraviglia, tutto presente, luminoso, agitato, e non per descrizione. Bravissimo».

54.E.C., Milano, 28 giugno 1968, lett. copia c. c. vel. ms.

55. E.C., Corvara, 29 luglio 1968, lett. copia c. c. vel. ms.

«La mia scoperta dell'America è stata straordinariamente interessante».

56. E.C., Milano, 11 agosto 1968, lett. copia c. c. vel. datt.

70 In una nota presente nel Fondo, Calzavara appuntava: «Poesia perfetta come Le sciat di Bodler [scherzosamente, per Le chat di Baudelaire] da fare col computer o no. Vedi in proposito Quel sabo de settembre di cui un verso fu rifatto 33 volte e poi tutta la poesia fu da me ripudiata. La poesia per me non deve nascere dalle parole ricche, ma piuttosto da quelle 'povere'. C'è più 'garanzia'. Non lasciarsi mai sfuggire l'occasione del tenero, semplice piacere di fare una carezza ad un gatto».

71 Calzavara cominciava a mandare le poesie che entreranno in Come se (Calzavara 1974). 
«Le mando queste nuove poesie (6) (Ne avrei un'altra lunga, quasi un poemetto per cantastorie: La storia de Meri, iniziata due anni fa, maturata poi in America, - quasi finita; gliela manderò un'altra volta)».

57. E.C., Milano, 7 ottobre 1968, lett. copia c. c. vel. datt.

«Ho finito il mese scorso la mia lunga filastrocca all'antica de $L a$ storia de Meri e gliene mando qui unita una velina».

58.E.C., Milano, 17 ottobre 1968, lett. copia c. c. 2 vell. datts.

«Per quanto riguarda La storia de Meri trovo giuste in gran parte le Sue osservazioni e ne terrò conto per l'aggiustamento».

59. E.C., Milano, 18 dicembre 1968, lett. copia c. c. vel. ms.

«In questi ultimi tempi non ho fatto molto. Solo pensato e riflettuto qualche momento».

60. M.C.M., Roma, 26 gennaio 1969, lett. f. ms. sf.

61. E.C., Milano, 10 febbraio 1969, lett. copia c. c. vel. ms.

«A questo punto mi sento trascinare dalla irresistibile tentazione di chiedervi consigli per la cernita, l'ordine e il montaggio dei pezzi di cui credo abbiate già quasi tutte le copie».

62. M.C.M., Roma, 13 febbraio 1969, lett. f. ms. sf.

«Di lui, ${ }^{72}$ belle e brutte, lettere e cartoline, ne abbiamo 302, e poi il resto».

63.E.C., Milano, 19 febbraio 1969, lett. copia c. c. vel. ms.

«È notevole il numero delle lettere di Comisso che voi avete. Un’altra raccolta?».

64. M.C.M., Roma, 5 marzo 1969, lett. f. ms. sf.

65.E.C., Milano, 7 marzo 1969, lett. copia c. c. vel. ms.

66. E.C., Milano, 15 marzo 1969, lett. copia c. c. vel. datt.

67. E.C., Milano, 13 aprile 1969, lett. copia c. c. vel. datt.

«Ho deciso di mutare tutta la struttura del libretto e soprattutto di non pubblicare (salvo qualche composizione) la seconda parte

72 Giovanni Comisso. 
del manoscritto (Poesie de 'na volta). Già fin da principio sentivo che c'era qualcosa che non andava nell'insieme naturalmente, ma ora mi sono convinto che le poesie della seconda parte non legano con quelle della prima».

68.E.C., Milano, 25 luglio 1969, lett. copia c. c. vel. ms. 69. M.C.M., Roma, 20 settembre 1969, lett. 2 ff. datts.

«Ora volevo invece consigliarla di riprendere il fascicolo delle poesie inedite, e di tentare nuovamente l'ordinamento, che non le è riuscito la prima volta».

70.E.C., Milano, 3 ottobre 1969, lett. copia c. c. vel. datt.

«Ho riflettuto e rifletto ancora sui Vostri suggerimenti circa una diversa composizione del mio nuovo libretto di poesie da pubblicare».

71. M.C.M., Roma, 9 ottobre 1969, lett. 3 ff. datts.

«Ho dovuto leggere tre o quattro volte la Meri prima di abituar$\mathrm{mi}$, io passatista, alla sua storia».

72.E.C., Milano, 20 febbraio 1970, lett. copia c. c. vel. ms.

«Quello che ormai temevo si è verificato: Mondadori non mi pubblica. L’appoggio di M. Sansone non ha servito». ${ }^{73}$

\section{Natale Mazzolà}

Avvocato e letterato trevigiano, collezionista di edizioni foscoliane, Natale Mazzolà vantava amicizie del calibro di Giovanni Comisso, Gino Scarpa e Arturo Martini. Il carteggio con Ernesto risale al 1965, e si interrompe nel 1975 con la morte di Mazzolà. In realtà, il carteggio è a tre punte, Ernesto, Maria e Natale, in quanto spesso uno richiama l'altro, anche se dal punto di vista quantitativo la mole maggiore consiste nelle lettere di e a Maria (alla cui scheda si rimanda): alla morte di Maria nel 1970 il marito trovò il plico delle loro lettere e propose al poeta la donazione alla Biblioteca di Treviso. Calzavara acconsentì con la clausola di lettura dopo la propria morte.

1. E.C., Milano, 27 maggio 1960, lett. copia c. c. vel. datt.

73 Si fa riferimento al primo tentativo di pubblicazione per l'Almanacco dello Specchio, per cui vedi Marco Forti. 
«Le Sue Cronache partigiane ${ }^{74}$ sono un libro interessante e avvincente per tutti, specialmente per coloro che come me vi riconoscono luoghi, persone e momenti [...]. Peccato che l'opera (che deve esserle costato fatica con tanta ricchezza e precisione di dati) non sia in vendita. La sua diffusione potrebbe giovare non solo agli studiosi ma anche a molti giovani e anziani per capire il prezzo della libertà, il valore della lealtà o della dignità, del sacrificio, il significato di essere o di non essere uomini».

2. N.M., Roma, 3 dicembre 1962, lett. f. ms. fotoc.

3. N.M., Roma, 15 marzo 1965, cart. post. ms. sf.

4. N.M., Roma, 30 marzo 1965, lett. f. datt.

5. N.M., San Felice Circeo, 6 ottobre 1965, cart. ill. ms. sf.

6. N.M., Circeo, 4 ottobre 1967, cart. post. ms. sf.

7. N.M., Circeo, 9 ottobre 1967, lett. f. ms. sf.

8. N.M., Roma, 4 dicembre 1968, cart. post. ms. sf.

9. N.M., Roma, 16 marzo 1969, lett. f. datt.

10. E.C., Milano, 20 marzo 1969, lett. copia c. c. vel. ms.

11. N.M., Roma, 23 agosto 1969, lett. f. datt.

«Scindere il libro $E$ e portarne una sola parte nella nuova edizione, non le pare efficace». ${ }^{75}$

12. N.M., Roma, 1 gennaio 1970, cart. post. ms. sf.

13. N.M., Roma, 13 febbraio 1970, cart. post. ms. sf.

14. E.C., Milano, 20 febbraio 1970, lett. copia c. c. vel. ms.

15.E.C., Milano, 16 marzo 1970, lett. copia c. c. vel. ms.

16. N.M., Roma, 18 marzo 1970, cart. post. ms. sf.

17. N.M., Roma, 11 giugno 1970, telegr.

18. E.C., Milano, 12 giugno 1970, telegr.

19. E.C., Milano, 13 giugno 1970, lett. copia c. c. 2 vell. mss.

20. N.M., Roma, 30 ottobre 1970, lett. f. ms. sf.

«In un cassetto di Maria ho trovato ben collocate per date le lettere Sue e quelle di lei [...]. Vorrei mandarle alla biblioteca in plico suggellato lasciando a Lei la scelta».

21. E.C., Milano, 16 novembre 1970, lett. copia c. c. vel. ms.

«Quello che Lei mi scrive mi commuove e mi onora. Se il carteggio Gaia-Felton che Lei ha trovato e che intende mandare alla Bibliote-

74 Il riferimento è a Mazzolà 1960 .

75 A Maria. Si tratta della divisione fra 'poesie nuove' (Parole mate) e 'poesie vecchie' (Parole pòvare), di cui è effettivamente composto il volume a stampa. 
ca di Treviso potesse interessare qualcuno, mi sembra più opportuno che sia letto dopo che me ne sarò andato anch'io».

22. N.M., Roma, 20 novembre 1970, cart. post. ms. sf.

«Pensavo anch'io quanto mi scrive e sarà fatto così».

23.E.C., Milano, 7 novembre 1972, lett. copia c. c. 3 vell. mss.

«Da tempo non ho Sue notizie che in vie frammentarie da comuni amici di Treviso quando ci vado».

24. N.M., Conegliano, 28 novembre 1972, cart. post. ms. sf.

«Donato l'appartamento di Roma agli Istituti di Ricovero di Treviso, sono ora ai piedi dei colli».

25.E.C., Milano, 11 dicembre 1973, lett. copia c. c. vel. ms.

«Desidero comunicarti che la stampa del mio libro Come se è a buon punto».

26. E.C., Milano, 19 marzo 1974, lett. copia c. c. vel. ms.

27. E.C., 15 giugno 1974, lett. f. ms. sf.

28. N.M., Treviso, 17 giugno 1974, lett. f. ms. sf.

«Passo un momento difficile che si ripercuote sul sistema nervoso [...]. Non sono adatto alla comunità, questo è tutto. Ho trovato il rimedio pur restando dove sono. Potremo rivederci, ma in via privata».

29. E.C., Milano, 15 dicembre 1974, lett. copia c. c. vel. ms.

«Spero che tu abbia letto sulla Fiera Letteraria [...] la lunga recensione di trecento lettere di F. Comisso in cui viene fatto largamente riferimento a Maria e a te». ${ }^{76}$

30.E.C., Treviso, 26 maggio 1975, lett. copia c. c. vel. ms.

31. E.C., Treviso, 8 novembre 1975, lett. copia c. c. vel. ms.

76 Armandi 1974 


\section{Mario Micozzi}

Micozzi ha legato il suo nome agli «Incontri nazionali tra la critica e la poesia» di Lanciano e alla rivista Punto d'incontro, fondata nel 1978 (lettere nrr. 11-12). Invitò Calzavara ad entrare nel Comitato scientifico della rivista, con Bàrberi Squarotti e Manacorda, ma Calzavara rifiutò, probabilmente perché non si riteneva sufficientemente competente.

1. M.M., Lanciano, 13 ottobre 1970, lett. f. ms. sf.

«A Lanciano presto nascerà una grande rivista che avrà per titolo "Punto d'incontro", incontro cioè di forze culturali di ogni estrazione politica e di diverse discipline. Tu sarai uno dei redattori. Va bene? Fanno parte del comitato della rivista: Squarotti, Manacorda, Pautasso, Montanelli, A. Levi, A. Parronchi, A. Bonsanti, Rosuto, Salinari e M. Corti. Quanto a quest'ultima, ti prego di invitarla a nome del comitato e per tuo caldo interessamento a far parte dirigente della rivista».

2. E.C., Milano, 14 ottobre 1970, lett. copia c. c. vel. ms.

«Ho telefonato subito a M. Corti. Mi ha detto innanzitutto che Vi ringrazia e che sarebbe lieta di collaborare, ma che è perplessa sulla sua adesione perché è molto impegnata col suo lavoro e specialmente con la sua rivista "Strumenti critici"».

3. M.M., Lanciano, 4 novembre 1971, lett. f. ms. sf.

«Ti ringrazio ancora una volta per il tuo interessamento nei riguardi della rivista "Punto d'incontro". So bene quanto stai facendo per far decidere la Corti a mandare uno suo scritto alla rivista».

4. E.C., Milano, 17 dicembre 1971, lett. copia c. c. vel. ms.

5. M.M., Lanciano, 21 aprile 1974, lett. f. ms. sf.

6. E.C., Sion, 29 aprile 1974, lett. copia c. c. vel. ms.

7. M.M., Lanciano, 9 maggio 1974, lett. f. ms. sf.

«Poesia in dialetto e poetiche moderne ${ }^{77}$ è una cosa pregevole, ricca di perspicaci e utili confronti, di citazioni opportune e mai esorbitanti, di considerazioni personali a volte originali. Ti ringrazio assai per avermelo spedito».

77 Calzavara 1971. Cf. Manlio Cortelazzo. 
8. E.C., Milano, 25 maggio 1974, lett. copia c. c. vel. ms.

«Ho incontrato l'altro giorno a un congresso Maria Corti e le ho fatto presente la tua lettera. [...] Mi ha detto però che risponderà a tutti e quindi si occuperà anche di te».

9. M.M., Lanciano, 28 giugno 1975, lett. f. ms. sf.

«Mi sono ormai convinto che [alcuni] critici non rispondono; hanno una specie di inedito-fobia congenita della quale - sono certo - non guariranno mai».

10. E.C., Vetriolo Terme, 21 luglio 1975, lett. copia c. c. vel. ms.

«D’altra parte c'è pure da tener presente, come mi diceva tempo fa un buon critico tra i più conosciuti che essi ricevono perfino due o tre pubblicazioni o manoscritti al giorno oltre le lettere, per cui diventa per loro praticamente impossibile una attenta lettura e una risposta a tutti».

11. M.M., Lanciano, 2 aprile 1979, lett. f. ms. sf.

«A rompere il silenzio sono io con l'annunciarti l'uscita di una rivista da me (modestamente) fondata, che, in breve, è riuscita a imporsi su piano nazionale con autorevolezza».

12.E.C., Milano, 20 maggio 1979, lett. copia c. c. vel. ms.

«Complimenti per la tua Rivista! Veramente ben fatta, ricca e soprattutto attendibile per il suo carattere interdisciplinare».

\section{Attilio Momigliano}

Il critico dimostrò di apprezzare la prima poesia di Calzavara, pubblicata nella prima delle tre plaquettes, Il tempo non passa, ${ }^{78} \mathrm{che} \mathrm{Cal}$ zavara fece stampare a sue spese in edizione numerata (Calzavara 1946). Le poesie sono in lingua. A partire dall'anno seguente, con l'uscita della seconda raccolta, I fiori di carta, Calzavara fece comparire le prime due poesie in dialetto, «Inverno» e «Can». L'anno dopo uscì la terza e ultima plaquettes, Il nuovo mondo, anch'essa chiusa da due poesie in dialetto, «I pavèri» e «La note de San Martin», prime prove di quello che qualche anno dopo diventerà un impegno 
pressoché totalizzante ${ }^{79}$ (Calzavara 1947, 1948).

1. A.M., Firenze, 20 aprile 1947, lett. 4 ff. mss. fotoc.

«Ma Il tempo non passa mi piace davvero [...]: c'è un sentimento forte, un pensiero grandioso, e un ritmo adatto a questa Sua poetica [...]. Mi duole non aver tempo di precisare meglio il mio pensiero. Ma sono lieto di dirLe, come sono solito, schiettissimamente le mie impressioni».

\section{Salvatore Nigro}

Nigro scrisse a Calzavara sull'intenzione di menzionarlo all'interno della Storia della Letteratura Italiana della Laterza, nel volume di aggiornamento al Novecento. Calzavara inviò materiale di studio e le trascrizioni dei seminari promossi da Bonora e Folena (a cui si rimanda).

1. S.N., Catania, 15 settembre 1980, lett. f. ms. sf.

«Conoscerà forse la Letteratura italiana. Storia e testi in 20 voll. dell'Editore Laterza. L'opera è già completa. Però stiamo preparando un volume di aggiornamento e di integrazioni al Novecento. E così ho pensato di dedicare un paragrafo alla sua poesia, soprattutto in dialetto».

2. E.C., Treviso, 25 settembre 1980, lett. copia c.c vel. ms.

«La ringrazio per avermi ricordato per il suo volume [...]. Penso tuttavia che il materiale che le invierò sarà sufficiente per il suo paragrafo».

3. S.N., Catania, 10 ottobre 1980, lett. f. ms. sf.

«La ringrazio della lettera e del suo pacco di libri e fotocopie. Mi metto subito al lavoro. Dovrò consegnare il libro a Laterza a metà gennaio. Prima dell'estate, quindi, dovrebbe uscire».

4. E.C., Milano, 20 ottobre 1980, lett. copia c. c. vel. ms.

5. E.C., Milano, 10 novembre 1980, lett. copia c. c. vel. ms.

79 Uscirono in Poesie dialettali, tranne «Inverno», che comparirà nella raccolta successiva e Parole mate Parole pòvare (Calzavara 1966). 
«Perché anche tu sei estimatore ed amico di Bonora e di Folena ti mando fotocopia delle loro brevi presentazioni (tutte da nastro) ai seminari tenuti quest’anno presso le loro facoltà».

6. E.C., Milano, 13 novembre 1980, lett. f. ms. sf.

7. S.N., Catania, 18 novembre 1980, lett. f. ms. sf.

8. S.N., 1984, cart. ill. ms. sf.

«Ho ricevuto e ho letto Le ave parole. Che dirti? È un libretto straordinariamente bello».

\section{Giovanni Orelli}

Romanziere e studioso di Lugano, cugino di Giorgio, ringraziò Calzavara per l'invio di Le ave parole.

1. G.O., Lugano, 13 aprile 1984, lett. f. ms. sf. [fig. 14]

«La ringrazio molto, di cuore, per avermi mandato (è stata una piacevolissima sorpresa!) e con dedica le Sue Ave parole. Mi scuso poi per il ritardo con cui le dico questo povero grazie. Perché (devo aggiungere) le poesie le ho lette subito, e rilette, e l'Homo praesens l'ho fatto a scuola con schietto piacere dei miei allievi [...]. Vorrei poi usare (se Lei non si oppone) i due versi de Le api del faraone "Non derubiamo i morti più di quanto / si derubano i vivi" come epigrafe per una Antologia delle Lettere nella Svizzera Italiana». ${ }^{80}$

2. G.O., Lugano, 16 marzo 1990, lett. f. ms. sf.

3. E.C., Milano, 26 marzo 1990, lett. copia c. c. vel. ms.

\section{Ivano Paccagnella}

Il 4 maggio 1980 uscì sul Giornale di Calabria l'articolo di Ivano Paccagnella «Analfabeto, versi in dialetto di Ernesto Calzavara. Parole... per salvarsi», che portò a una riflessione del poeta sulla propria poesia.

1. I.P., 4 marzo 1980, lett. f. ms. sf.

«Le prometto, infine, una recensione del suo libro in una zona lontana, sul Giornale di Calabria». 
2. E.C., Milano, 15 marzo 1980, lett. copia c. c. 2 vell. mss.

«Bonora tempo addietro mi parlò di Lei con convinzione. Ma Lei intanto mi ha regalato alcune ore d'una lettura che mi ha vivamente interessato. Peccato che la mia limitata competenza filologica e linguistica non mi permetta di valutare in toto l'importanza specifica di alcune fra le sue pagine più specializzate».

3. E.C., Milano, 22 maggio 1980, lett. 2 ff. mss. fotoc.

«La ringrazio di quanto Lei ha spiegato sulla mia poesia, sul suo naturale sviluppo evolutivo specialmente dal primo all'ultimo testo della mia piccola trilogia. Ė verissimo, per molte ragioni, che non ha molto senso voler a tutti i costi inserire $C$. in una linea di poesia dialettale veneta. Degli autori che stanno dietro ad Analfabeto quanto a Tessa fino a un certo punto (in Poesie dialettali) è vero, ma dopo $E$ gli ho camminato solo qualche volta parallelamente a molti diversi piani, ma senza veramente incontrarmi con lui. Lucini letto di sfuggita in questi ultimi anni in qualche raccolta non $\mathrm{mi}$ ha mai lasciato alcuna traccia. Con la linea lombarda può darsi che abbia qualche lontana affinità riflessa per l'influenza più o meno determinante del microclima lombardo in cui vivo da molti anni più di frequente. Con Noventa non ho assolutamente nulla da spartire anzi sono forse in antitesi: giustamente Lei in proposito ha detto esperienza più cosmica e meno scettica. Veramente più che altro, ho sentito, pur conservando le mie caratteristiche personali, simpatia per la poesia e le poetiche dell'area atlantica [...]. Ed è una esperienza questa che nella poesia dialettale italiana, non $\mathrm{mi}$ sembra, salvo errore, sia stata mai fatta finora da altri».

\section{Ferdinando Palmieri}

Studioso, sostenitore e scrittore di testi in dialetto, inviò a Calzavara parole di apprezzamento dopo l'invio di e Parole mate Parole pòvare.

1. F.P., 28 gennaio 1967, lett. copia c. c. vel. datt.

«Molte e molte grazie del ricordo e del libro. Il quale libro è veramente un dono preziosissimo di poesia».

\section{Geno Pampaloni}

Il 19 settembre 1974 Pampaloni scrisse per Il Mondo un articolo dal titolo (e sottotitolo) «In un angolo della letteratura. Ė un poeta, scu- 
satelo. Nella cultura di oggi, la poesia appare stritolata da forze troppo più potenti di lei. Eppure non è una poesia solitaria, ma gremita, popolosa, sollecita, stanza senza imposte dove vorticano spifferi e venti gelidi», sulla situazione della poesia italiana, e in cui è citato anche Calzavara. Nel 1984 commentò i versi contenuti ne Le ave parole, mandati in dono dal poeta qualche giorno prima.

1. G.P., Firenze, 31 marzo 1984, cart. post. ms. sf.

«I suoi versi si compongono in un'armonica libertà espressiva dalle molte soluzioni».

\section{Pier Paolo Pasolini}

Nel 1966 Calzavara aveva in progetto l'istituzione di un nuovo premio letterario, denominato «La barchessa», da tenersi a Levico. Nel fondo sono presenti degli appunti di quell'anno che portano l'indicazione «Abbozzo del Bando di concorso della nuova poesia dialettale veneta» insieme ad un «Abbozzo per l'istituzione di un concorso della nuova poesia dialettale veneta, denominato La barchessa». Calzavara conosceva bene il paese, in quanto mèta costante dei suoi soggiorni estivi, frequentato per lungo tempo anche per motivi di salute. Al progetto partecipava anche Sandro Zanotto, che gli comunicava come Scheiwiller fosse dell'idea di far aderire all'iniziativa sia Pasolini sia Zanzotto, e che a questo scopo li avrebbe contattati personalmente (vedi Sandro Zanotto, lettere nrr. 1-2). La lettera che Calzavara inviò a Pasolini contiene la richiesta di partecipazione alla nascente iniziativa. Tuttavia il progetto non ebbe seguito. Come si intuisce dalla lettera, poi, Pasolini conosceva già Calzavara: leggeva la sua poesia già dal 1946, dopo la pubblicazione delle tre plaquettes in lingua. Come Calzavara raccontava in una lettera del 1985 inviata a Mario Chiesa, Pasolini gli aveva scritto subito dopo averle lette (vedi Mario Chiesa, lettera nr. 23).

1. E.C., Milano, 13 novembre 1966, lett. copia c. c. vel. datt.

«Dopo tanti anni dal nostro primo incontro epistolare, mi faccio vivo ancora con Lei per la poesia veneta».

\section{Giuseppe Pontiggia}

Pontiggia scrisse la recensione apparsa sul Corriere della Sera dal titolo «Fascino di un titolo più che di una filosofia. Il mondo si regge su un Come se», il 3 settembre 1980. Curò con Marco Forti il nr. 7 
dell'Almanacco dello Specchio dedicato alla poesia dialettale, con una sezione su Calzavara (vedi Marco Forti).

1. G.P., Milano, 27 novembre 1984, lett. f. ms. sf. [figg. 15-16]

«Grazie della dedica e soprattutto del libro: ${ }^{81}$ leggerlo è come una avventura vivificante, un viaggio dentro gli strati del linguaggio, con incursioni in un latino reso improvvisamente vivo dalla contiguità delle altre lingue».

2. E.C., Milano, 12 dicembre 1984, lett. f. ms. sf.

3. G.P., Milano, 12 marzo 1990, lett. f. ms. sf.

«E un grande libro, ${ }^{82}$ grande per l'orizzonte storico e umano, e grande per la varietà, la ricchezza e la potenza espressiva».

4. E.C., Milano, 18 marzo 1990, lett. copia c. c. vel. ms.

«Tu puoi ben comprendere quanto siano necessarie la partecipazione e la solidarietà di coloro che stimiamo in modo particolare come io stimo te».

\section{Antonio Porta}

Il 29 luglio 1979 uscì sul Corriere della Sera l'articolo di Porta dal titolo «Scelte al di fuori delle false definizioni e degli steccati generazionali. Quattro poeti per una nuova collana» che toccava, seppur marginalmente, la poesia di Calzavara contenuta in Analfabeto, e il 22 agosto 1984 sullo stesso quotidiano una recensione a Le ave parole, dal titolo «Una raccolta di Ernesto Calzavara. Quel dolce ronzio delle parole». Per entrambi Calzavara espresse a Porta il proprio apprezzamento, nonostante in una lettera ad Agosti si dicesse non del tutto d'accordo sulle posizioni di Porta (vedi Stefano Agosti, lettera nr. 11).

1. E.C., Milano, 9 agosto 1979, lett. copia c. c. vel. ms.

«Ho letto in questi giorni il tuo articolo sul Corriere che riguarda anche il mio Analfabeto. Molto giusto quello che tu dici nelle premesse generali. Ci voleva. Per il resto sono contento di come hai guardato il mio libro, sia pure nell'ambito ristretto che ti era consentito dal breve ma importante articolo».

81 Calzavara 1984

82 Calzavara 1990 
2. E.C., Treviso, 24 agosto 1984, lett. copia c. c. vel. ms.

«Ti ringrazio molto di esserti fatto così felicemente intermediario della mia poesia col pubblico del Corriere».

\section{Giovanni Pozzi}

Calzavara inviò a Pozzi, esperto di poesia visiva, ${ }^{83}$ il Cembalo scrivano (Calzavara 1977; vedi lettera nr. 3), nonostante l'insoddisfazione per il risultato ottenuto, riferendo l'intenzione di pubblicare una seconda edizione del libro (che non ci fu mai). Nel 1980 lo studioso organizzò un seminario di analisi di testi poetici tenuto da due suoi allievi, Marco Guaita e Luisa Foletti, dell'Università di Friburgo (vedi la lettera nr. 5 di Angelo Zanon Dal Bo). Una delle lezioni è dedicata all'analisi della poesia «Tuti i mati fa i so ati» (conservata in $15 \mathrm{ff}$. datts.).

1. E.C., Milano, 5 dicembre 1977, lett. copia c. c. vel. datt.

«La Sua fama di illustre studioso italianista, la conosco dall'amico Cesare Segre».

2. G.P., Friburgo, 18 dicembre 1977, lett. f. ms. sf.

3. E.C., Milano, 22 marzo 1978, lett. 2 ff. mss. sf.

«Le mando questo mio piccolo divertissement per quanto [...] non sia proprio come avrei voluto».

4. E.C., Milano, 23 marzo 1978, lett. copia c. c. vel. ms.

5. G.P., Friburgo, 14 maggio 1978, lett. f. ms. sf. [fig. 17]

«La ringrazio moltissimo dell'invio [...] del delizioso Cembalo. Sto studiando i carmi figurati nelle loro varie manifestazioni ed il Suo invio colpisce diritto il cuore dei miei attuali interessi».

6. E.C., Milano, 21 maggio 1978, lett. copia c. c. vel. ms.

«E probabile che io ne rifaccia una seconda edizione migliorata in tutto il suo complesso (titoli non più sopra i blocchi dei dattilogrammi, che dovrebbero rimanere isolati, ma sulla facciata bianca o sinistra in fondo e forse... in una lingua antica anziché in dialetto. Ma ci debbo riflettere)». 
7. G.P., Friburgo, 10 luglio 1978, lett. f. ms. fotoc.

8. G.P., Friburgo, [1980], lett. f. ms. sf.

9. G.P., Friburgo, 3 gennaio 1981, lett. f. ms. sf.

10. E.C., Milano, 4 dicembre 1981, lett. copia c. c. vel. ms.

11. E.C., Milano, 13 dicembre 1981, lett. copia c. c. vel. ms.

12.E.C., Milano, 22 dicembre 1981, lett. copia c. c. vel. ms.

«Ricco di penetrazione e d'illustrazione, di notizie e di riferimenti, esauriente e chiaro, La parola dipinta è uno dei pochi libri di studio e di consultazione necessari e destinati a rimanere testi. Un unicum in materia».

13.E.C., Milano, 5 marzo 1982, lett. f. ms. sf.

14. E.C., Milano, 12 marzo 1983, lett. f. ms. sf.

15.E.C., Vetriolo Terme, 8 luglio 1984, lett. copia c. c. vel. ms.

«Ho ricevuto tempo fa a Milano il suo saggio sul Cantico di Frate Sole ${ }^{84}$ [...]. Il suo dono è stato un invito ad entrare nella Casa del Santo per sentirlo e conoscerlo meglio nella sua religiosità».

16. G.P., Lugano, 11 giugno 1990, lett. f. ms. sf.

\section{Massimo Rizzante}

Rizzante curò la pubblicazione di un numero monografico della rivista Baldus, «Per Ernesto Calzavara» (cf. Rizzante 1993). ${ }^{85}$ L'intervista di cui si dice nella lettera nr. 3 compare nella rivista. Rizzante fu anche il curatore dell'ultima opera di Calzavara, Rio terrà dei pensieri, costituita di testi in prosa, poesia e aforismi (lettera nr. 10).

1. M.R., Parigi, 16 aprile 1992, lett. f. datt.

«Sarebbe ora che la sua poesia venisse letta al di là della problematica dialettale. Gli sta stretta».

2. E.C., Treviso-Milano, 12 maggio 1992, lett. copia c. c. f. ms.

84 Si tratta di uno dei saggi di Pozzi sul Cantico; una delle sedi più prestigiose in cui pubblicò in materia fu Pozzi 1992. Nel Fondo è conservata la bozza di un saggio di Pozzi dal titolo Rileggendo il Cantico di Frate Sole, che Pozzi aveva inviato a Calzavara per la lettura (la data di mano di Calzavara è 5 giugno 1984).

85 Testi di Massimo Rizzante, Cesare Segre, Fabio Franzin, Gian Mario Villalta, Lello Voce, Biagio Cepollaro, Sandro Zanotto, Andrea Zanzotto. Inediti di Ernesto Calzavara e traduzioni in inglese, francese e friulano di Hermann W. Haller, Philippe Di Meo e Amedeo Giacomini. 
3. M.R., Parigi, 1 giugno 1992, lett. f. datt.

«Ciò che io e i miei amici vogliamo fare è ricondurre il suo discorso poetico entro le maglie della poesia, superando i confini della poesia dialettale. Avrei anche l'intenzione, se lei è d'accordo, di stilare un'intervista in grado di delineare un percorso letterario e biografico».

4. M.R., Parigi, 29 gennaio 1993, lett. f. ms. sf.

5. M.R., Monosque, 14 settembre 1993, cart. ill. ms. sf.

6. M.R., Jesolo, 27 dicembre 1993, lett. f. ms. sf.

7. E.C., Milano, 26 gennaio 1994, lett. copia c. c. f. ms.

8. M.R., 27 gennaio 1994, lett. f. ms. sf.

«In ogni caso è un bel risultato che un giovane ottantenne venga accolto con entusiasmo e interesse da alcuni giovani trentenni, poeti, critici, forse tra i migliori».

9. E.C., Milano, 12 febbraio 1994, lett. copia c. c. f. ms.

10. E.C., 27 febbraio 1994, lett. f. ms. sf.

«Ti ho incaricato di sistemare nel modo più opportuno il materiale della mia nuova opera letteraria Rio terrà dei pensieri».

11.E.C., Milano, 2 marzo 1994, lett. copia c. c. f. ms.

\section{Giorgio Ronconi}

Il critico lesse a apprezzò le poesie di Calzavara.

1. G.R., Padova, 13 dicembre 1979, lett. f. ms. sf.

«Ho ricevuto nei giorni scorsi il suo Analfabeto, che ho scorso tutto e letto qua e là con attenzione e con vivo interesse e diletto».

2. E.C., Milano, 22 dicembre 1979, lett. copia c. c. vel. ms.

3. G.R., Padova, 21 gennaio 1981, lett. f. ms. sf.

«Le auguro di star sempre bene, e di continuare a produrre con quella fertile vena che conosciamo e ammiriamo. Leggendo Folengo, per una tesi che ho in corso, mi sovvengono i suoi versi, e non stonano».

4. E.C., Milano, 28 aprile 1984, lett. f. ms. sf. 


\section{Max Roqueta}

Roqueta ricevette da Calzavara una lettera di apprezzamento per i suoi versi, in cui è sottolineata l'importanza di coltivare le varietà linguistiche locali.

1. E.C., Milano, 27 giugno 1977, lett. copia c. c. vel. datt.

«Ho letto con interesse i Suoi versi. ${ }^{86}$ Mi sono piaciuti la loro vivacità, il vigore, l'immaginazione e soprattutto la forza e l'abilità con cui Lei ha saputo innestare sulla poesia tradizionale del Suo paese una poesia originale moderna, piena di vibrazione e di passione. Ho apprezzato la Sua tecnica, il Suo discorso, la calda attualità e profondità delle Sue idee, la Sua bella lingua occitanica che ha risonanze affini anche alla mia lingua veneta. Lo spirito della terra dove siamo nati deve essere salvato prima che esso venga sommerso dalla marea montante della civiltà meccanica e consumistica. Lo si difende prima di tutto coltivando i linguaggi locali e i dialetti. La nuova Europa si difende ancora sulle mura antiche delle nostre piccole città. Sono tornato entusiasta dal Suo Midì. Ė uno dei paesi più belli del mondo che mi è caro per gli antichi rapporti dei suoi poeti con la mia Treviso medioevale e per qualche affinità di carattere tra le due terre».

\section{Giuseppe Rosato}

Poeta, scrittore, giornalista e collaboratore di programmi culturali per la RAI, Giuseppe Rosato fece parte dell'organizzazione del «Premio Lanciano» di poesia dialettale, vinto da Calzavara nel 1967 (vedi la scheda di Mario Micozzi).

1. G.R., Pescara, 11 novembre 1977, lett. f. datt.

«A Lanciano tutto è finito, miseramente spento dall'incuria dei nuovi amministratori. I dieci anni del Premio Lanciano restano sì un fatto ricordevole, ma sarebbe stato assai meglio farla durare, quella iniziativa, che poi con il relativo apparato dei Convegni sul dialetto era diventato cosa rara, unica in Italia».

2. E.C., Milano, 27 novembre 1977, lett. copia c. c. vel. ms.

«Il mio lavoro professionale è molto meno intenso di una volta [...]. Tuttavia ho avuto qualche soddisfazione: articoli, riferimen-

86 Forse Roqueta 1974-75. 
ti, presentazioni [...]. In questi giorni è uscita con Scheiwiller una mia plaquette di poesie diverse: Cembalo scrivano Esercizi per dattilogrammi che purtroppo dal punto di vista tipografico, non è riuscito come doveva [...]. Pur tuttavia continuo a restare dentro la ricerca che mi conforta quando mai sembra di essere sulla buona strada, ma non mi dà mai del tutto appagamento e tranquillità».

3. G.R., Pescara, 20 febbraio 1990, lett. f. datt.

«Ricevo il tuo bel volume, giusto coronamento di una vita dedicata alla poesia, e ne godo intimamente».

4. E.C., Milano, 4 marzo 1990, lett. copia c. c. vel. ms.

\section{Mario Sansone}

La corrispondenza con Mario Sansone prese avvio con le vicende legate ad una possibile pubblicazione con Mondadori, che non si realizzerà (vedi Marco Forti). Successivamente, nel 1973, Calzavara partecipò al Premio Nazionale di Poesia «Premio David» con le poesie «Le Onorevoli Amanti», «I contratti d'autunno», «Dopo il notevole benessere», che confluiranno in Come se (Calzavara 1974). Calzavara venne selezionato nella rosa dei vincitori; fra i componenti della commissione vi era anche Mario Sansone, che gli inviò il telegramma con la comunicazione del premio.

1. E.C., Milano, 10 aprile 1969, lett. copia c. c. vel. datt.

«Le mando, come d'accordo, fotocopie di recensioni e di articoli sul mio e insieme a una copia del libretto affinché Lei possa dare tutto a Forti nell'incontro che avrà prossimamente con lui».

2. M.S., Bari, 1 maggio 1969, cart. post. ms. sf.

«Parlai con Marco Forti e gli consegnai i suoi versi. Egli mi prospettò alcune difficoltà editoriali».

3. E.C., 7 luglio 1969, lett. f. ms. sf.

4. M.S., Roma, 9 luglio 1969, lett. f. ms. sf.

«Sono lieto che il suo lavoro abbia...passato il primo esame».

5. M.S., Capri, 15 luglio 1969, lett. f. ms. sf. 
«Ho buona fiducia che tutto andrà a buon fine e che Lei sarà finalmente laureato poeta - con merito - anche per il grande pubblico».

6. M.S., Capri, 23 luglio 1969, lett. f. ms. sf.

7. E.C., La Villa in Badia, 31 luglio 1969, lett. f. ms. sf

«Le sono veramente molto e molto grato di quanto Lei ha fatto». ${ }^{87}$

8. M.S., Napoli, 1 giugno 1970, cart. post. ms. sf.

9. M.S., Roma, 30 dicembre 1970, cart. post. ms. sf.

10. M.S., Marina di Carrara, 7 settembre 1973, telegr.

«Lieto comunicarle commissione premio David habet incluso suoi componimenti rosa sei segnalati cerimonia premiazione avrà luogo domani».

11. M.S., Roma, 7 novembre 1977, cart. post. ms. sf.

«Lei sa quanto La stimi e quanto sono orgoglioso di averne dato, credo per primo, pubblico riconoscimento!».

12.M.S., Roma, 9 dicembre 1977, cart. ill. ms. sf.

\section{Gregorio Scalise}

Scalise esordì come poeta negli anni Sessanta con tre plaquettes di poesia visiva sperimentale, pubblicate nelle edizioni torinesi Geiger di Adriano Spatola (alla cui scheda si rimanda), A capo, L'erba al suo erbario e L'uomo nell'astuccio, la prima del 1968, le altre due del 1969. I due si scambiarono informazioni sulle fonti filosofiche comuni.

1. G.S., Bologna, 13 aprile 1978, lett. f. datt.

«Sto cercando Vahinger, ma non lo trovo [...]. Negli appunti che gentilmente hai scritto a Milano, trovo anche un altro nome. Nathorp. Si tratta della scuola neokantiana? Sono per me informazioni molto utili».

2. E.C., Milano, 19 aprile 1978, lett. copia c. c. vel. datt.

87 Si riferisce all'intercessione per la pubblicazione sull'Almanacco dello Specchio, per cui vedi Marco Forti. 
«Ti avverto però che il mio campo non è la filosofia e che quella del Come se, che ha dato il primo titolo del libro c'è entrata di sfuggita, solo per la composizione omonima che mi fu ispirata da poche righe dell'Abbagnano sull'argomento, molto dopo completata con una breve lettura di Veihinger. Per tutto il resto i motivi di fondo della mia ricerca hanno un'altra impostazione del tutto personale».

\section{Giuseppe Scarpa}

Il carteggio tra i due amici rivela il lato umano di Calzavara. Entrambi scrittori, si scambiarono opinioni letterarie; sono particolarmente sentiti i racconti di vita vissuta narrati nelle lettere.

1. G.S., Oderzo, 12 ottobre 1979, lett. 2 ff. mss. sf.

«Dopo aver faticato una vita intera per farmi un nido per la vecchiaia ormai in atto, mi trovo sbattuto fuori casa».

2. G.S., Oderzo, 13 ottobre 1979, lett. 2 ff. mss. sf.

3. E.C., Milano, 19 ottobre 1979, lett. copia c. c. vel. ms.

«In questa vicenda ho ammirato il tuo sobrio distacco di gentiluomo e poi la ennesima riprova della tua invidiabile capacità scrittoria nella resa delle situazioni e degli ambienti. Ma perché non ti metti a scrivere un libro di ricordi?».

4. G.S., Oderzo, 27 ottobre 1979, lett. 3 ff. mss. sf.

«Sto mettendo a ferro e fuoco la bibliotechina della casa che annovera opere non disprezzabili, e soprattutto sto scrivendo le mie memorie che, almeno per me, appaiono interessantissime».

5. G.S., Oderzo, 21 novembre 1979, lett. 2 ff. mss. sf.

«A proposito di antichi ricordi, penso che ti farà piacere avere una tua foto scattata sul rio Fontanelle durante una delle tue sedute post-natatorie».

6. E.C., Milano, 27 novembre 1979, lett. copia c. c. vel. ms.

«Non ti so dire quanto mi abbia colpito quella foto di lontani tempi».

7. G.S., Oderzo, 18 gennaio 1980, lett. f. ms. sf. 
«Il Bepi Scarpa ha ripreso a scrivere le sue memorie! Memorie che, almeno per una non cospicua parte, saranno rese di pubblico dominio solo quando me ne starò rintanato per un po' di tempo nella quarta o quinta dimensione».

8. G.S., Oderzo, 31 gennaio 1980, lett. 3 ff. mss. sf.

9. E.C., Milano, 3 febbraio 1980, lett. copia c. c. vel. ms.

«Giorni fa ho ricevuto una tua lettera con la quale mi dicevi che avevi ripreso a scrivere il libro dei ricordi. Ne sono molto lieto perché penso che ora lo scrivere farà ciò che servirà a tenerti in forma sotto diversi aspetti».

10. E.C., Milano, 9 febbraio 1980, lett. copia c. c. 2 vell. mss.

«Quanto al sistema nervoso dei cani centocinquanta volte più sviluppato dell'uomo non capisco come mai non sia saltato fuori un Einstein cane e non sappiano contare almeno più di dieci».

11. G.S., Oderzo, 14 febbraio 1980, cart. ill. ms. sf.

12.G.S., Oderzo, 16 febbraio 1980, lett. 2 ff. mss. sf.

«Avrei da scrivere un volume sulla premonizione dei cani che comunque penso siano molto ma molto più umani della nostra civilissima razza umana [...]. Ti mando ad ogni modo uno stralcio delle mie Memorie che, per quanto purgate e limitate alla fine della Seconda Guerra Mondiale, devono aver suscitato malumori tra il parentume che, secondo quanto mi è stato riferito, non gradirebbero che venissero scoperti altarini di nessuna guerra anche a distanza di dieci lustri».

13.E.C., Milano, 23 febbraio 1980, lett. copia c. c. 3 vell. mss.

«Tu sai bene che per me gli animali (e per altri versi le piante) costituiscono veramente l'indispensabile ponte (le mie e) tra il mondo animato e quello inanimato delle pietre e dei minerali - senza dei quali la terra sarebbe come la luna».

14. G.S., Oderzo, 1 marzo 1980, lett. f. ms. sf.

«Hai perfettamente ragione per quanto riguarda le mie memorie».

15. G.S., Parco di Villa Varda, 9 maggio 1980, lett. 4 ff. mss. sf.

16. E.C., Milano, 21 maggio 1980, lett. copia c. c. vel. ms.

«Ho ricevuto la tua straordinaria lettera (che dovrebbe andare anch'essa tra gli atti delle tue memorie, se vuoi te ne faccio fotocopia)». 
17. G.S., Crosetta Cansiglio, 7 agosto 1980, lett. f. ms. sf.

«Tra gli intatti silenzi notturni la prosa (e penso la poesia) esce di getto come freschissima polla d'acqua montanina».

18. G.S., Oderzo, 23 ottobre 1980, lett. f. ms. sf.

19. E.C., Milano, 12 novembre 1980, lett. copia c. c. vel. ms.

20. G.S., Oderzo, 23 novembre 1980, cart. ill. ms. sf.

21. G.S., Oderzo, 28 novembre 1980, lett. 2 ff. mss. sf.

22. E.C., Milano, 23 dicembre 1980, lett. copia c. c. vel. ms.

«Purtroppo quest'anno che se ne va è stato per te e per me molto burrascoso per la nostra salute».

23. G.S., Oderzo, 1 gennaio 1981, lett. 2 ff. mss. sf.

24. G.S., Oderzo, 11 gennaio 1981, lett. f. ms. sf.

25. E.C., Milano, 18 gennaio 1981, lett. copia c. c. vel. ms.

«Devi però proseguire le tue scritture perché altrimenti sarebbe una piccola inammissibile vigliaccheria di fronte a te e agli interessi storico culturali e del costume della nostra comunità».

26. G.S., Oderzo, 23 gennaio 1981, lett. 2 ff. mss. sf.

«È vero che ho interrotto da qualche tempo la stesura delle memorie vere e proprie ma, in compenso, ho buttato giù qualche cosa».

27. E.C., Milano, 4 febbraio 1981, lett. copia c. c. vel. ms.

28. G.S., Oderzo, 7 febbraio 1981, lett. f. ms. sf.

29. G.S., Oderzo, 28 marzo 1981, lett. f. ms. sf.

«Per quanto concerne le mie memorie, l'uomo nato stanco [...] pensa di seguire il tuo consiglio e di redigerle sotto forma di romanzo».

30. G.S., Oderzo, 30 aprile 1981, lett. 2 ff. mss. sf.

31. E.C., Milano, 9 maggio 1981, lett. copia c. c. vel. ms.

32. G.S., Oderzo, 15 maggio 1981, lett. 2 ff. mss. sf.

33. E.C., Milano, 20 maggio 1981, lett. copia c. c. vel. ms.

34. E.C., 27 maggio 1983, lett. copia c. c. 2 vell. mss.

35. G.S., Treviso, 2 maggio 1984, lett. 2 ff. mss. sf.

«Sono convinto che ogni giorno passato in vita, nella situazione in cui mi trovo, serve a liberarmi dalle scorie accumulate nel mio spirito in quasi settantotto anni».

36. G.S., Treviso, 29 maggio 1984, cart. ill. ms. sf. 
37. G.S., Treviso, 31 maggio 1984, lett. 2 ff. mss. sf.

38. G.S., Treviso, 4 gennaio 1985, cart. ill. ms. sf.

39. G.S., Treviso, 21 gennaio 1985, lett. 2 ff. mss. sf.

40. E.C., Milano, 5 febbraio 1985, lett. copia c. c. vel. ms.

41. E.C., 13 febbraio 1985, lett. copia c. c. vel. ms.

42. G.S., Treviso, 17 maggio 1985, lett. 2 ff. mss. sf.

\section{Vanni Scheiwiller}

Con Scheiwiller Calzavara ebbe un rapporto privilegiato. Nel 1951 Vanni cominciò ad occuparsi della casa editrice ereditata dal padre, con un occhio di riguardo nei confronti della poesia dialettale di Tessa, Noventa, Marin, Giotti, e anche di Calzavara. Si riportano anche le belle lettere che Scheiwiller mandava ai critici per la promozione dei volumi, conservate nel Fondo. Le ultime lettere fra l'editore e il poeta sono incentrate sulla pubblicazione della raccolta antologica Ombre sui veri (Calzavara 1990).

1. E.C., Milano, 19 ottobre 1965, lett. f. datt.

«Le mando questo nuovo gruppo Secondo racolto di poesie dialettali un po' alla rinfusa, quasi tutte degli ultimi anni, pregandoLa di dirmi se questa volta, diversamente dalla precedente, possiamo metterci d'accordo per la loro pubblicazione. Come vedrà, la prima parte della raccolta contiene composizioni d'intonazione alquanto diversa dalla linea tradizionale e anche da quella ultima dei dialettali [...]. Il titolo naturalmente è suscettibile di mutamento. Per es. Ogni spin fa siesta o altro». ${ }^{88}$

2. E.C., Vetriolo Terme, 2 agosto 1966, copia c. c. vel. ms.

3. E.C., Milano, 10 dicembre 1970, lett. copia c. c. vel. ms.

4. V.S., Milano, 8 giugno 1971, lett. copia c. c. vel. ms.

5. V.S. a Giancarlo Vigorelli, Milano, 8 marzo 1972, lett. copia c. c. f. ms.

«Ora ti scrivo per un manoscritto che mi sta particolarmente a cuore e che vorrei tanto vederlo pubblicato prima possibile nella tua collana di Guanda».

6. E.C., Milano, 3 novembre 1973, copia c. c. 2 vell. datt. e ms.

7. V.S. a Premio Alte Ceccato, Milano, 6 luglio 1974, lett. copia c. C. f. ms.

88 e Parole mate Parole pòvare è effettivamente la prima raccolta pubblicata da Scheiwiller. 
8. V.S. a Nantas Salvalaggio, Milano, 6 luglio 1974, lett. copia c. c. f. ms.

9. V.S. a Alberico Sala, Milano, 6 luglio 1974, lett. copia c. c. f. ms.

10. V.S. a Bortolo Pento, Milano, 6 luglio 1974, lett. copia c. c. f. ms.

11. V.S. a Ferruccio Mazzariol, Milano, 6 luglio 1974, lett. copia c. c. f. ms.

«Sto annegando nelle mie carte e le devo sempre una risposta al suo progetto. Ci metto tanti anni: cinque anni per realizzare $\mathrm{Co}$ me se di Ernesto Calzavara».

12.V.S. a Enrico Niccolini, Milano, 6 luglio 1974, lett. copia c. c. f. ms. 13.V.S. a Giuliano Menato, Milano, 6 luglio 1974, lett. copia c. c. f. ms. 14. V.S. a Renata Ghiotto, Milano, 6 luglio 1974, lett. copia c. c. f. ms.

«È un poeta eccellente non solo veneto: e il premio ritengo serva solo a dare un piccolo compenso che un piccolo editore non è in grado di dare».

15. V.S. a Elio Filippo Accrocca, Milano, 6 luglio 1974, lett. copia c. c. f. ms.

«Ogni tanto ti invio dei libri di poesia: oggi è la volta dell'eccellente Ernesto Calzavara, Come se».

16. V.S. a Ugo Fasolo, Milano, 6 luglio 1974, lett. copia c. c. f. ms.

«Per il Premio, spero solo che un qualche premio possa compensare l'autore, che io ritengo un poeta eccellente».

17. V.S. a Giuseppe Mazzariol, Milano, 6 luglio 1974, lett. copia c. c. f. ms. 18.V.S. a Giorgio Caproni, Milano, 6 luglio 1974, lett. copia c. c. f. ms.

«Per espresso desiderio dell'A. invio al Premio Alte Ceccato Come se di Ernesto Calzavara, che io ritengo eccellente».

19. V.S. a Agenzia ANSA Vicenza, Milano, 28 ottobre 1974, lett. copia c. c. vel. datt.

20.V.S. a Il Gazzettino, Milano, 29 ottobre 1974, lett. copia c. c. $2 \mathrm{ff}$. datts.

21. V.S. a Il Gazzettino, Milano, 19 novembre 1974, lett. copia c. c. vel. ms.

22.E.C., Esino Lario, 6 gennaio 1976, lett. copia c. c. vel. ms. 
«Ho appena finito di rileggere [...] l'importante bellissimo volume del Calza: ${ }^{89}$ L'incanto sottile del dramma NÕ. La principessa Aoi. Devo proprio dire che ne ho avuto una impressione molto forte. Un lavoro sostanzioso e sostanziale».

23.E.C., Levico, 14 luglio 1976, lett. copia c. c. vel. ms.

24. E.C., Milano, 1 luglio 1979, copia c. c. vel. datt.

25.E.C., Milano, 21 aprile 1986, lett. 2 ff. datts.

«Ti mando con la presente due raccoglitori contenenti il materiale per la pubblicazione della Trilogia della e [...]. Tra noi da tempo non c'è mai stato modo di parlarci con un po' di calma. Tra l'altro per me, cinque operazioni, degenze, convalescenze ecc. E tu hai sempre tanta fretta. A fine maggio altra operazione. Ora mi tengo su a forza di cure e di medicine speciali, alcune delle quali, se mi aiutano a sopravvivere (ironia della sorte, mi sono perfino un po' ingrassato), arrestando l'avanzare della malattia, dall'altro mi danno una grande invincibile astenia. Sono da tempo in cura da un illustre clinico urologo della Università, ma la situazione non solo non migliora, ma si aggrava poco a poco. Io temo sempre più, soprattutto stante la mia età. Sono quasi ottanta e ben pochi dei miei vecchi sono riusciti a superarla. Aspettare ancora due o più anni [...] è un rischio per me evidente e lo sarebbe un po' anche per Voi, dato che avete accettato di pubblicarmi per l'86. Non ti scrivo per indurti a compassione. Ti prego quindi con tutte le mie più calde raccomandazioni, di darmi ascolto».

26. V.S., Milano, 10 giugno 1987, lett. f. datt. fotoc. [fig. 18]

27. V.S., Milano, 26 ottobre 1987, lett. f. datt.

28.E.C., Milano, 1 maggio 1988, lett. copia c. c. vel. datt.

29. E.C., Milano, 24 febbraio 1988, lett. copia c. c. vel. datt. e ms. sf. e mt.

«Qualunque possa essere l'esito e in qualsiasi evenienza confido sempre nella tua parola affinché entro la primavera dell'anno venturo al più tardi, secondo i nostri accordi, la mia antologia venga pubblicata».

30.E.C., Milano, 10 dicembre 1988, lett. f. datt. fotoc.

«Della terna dei titoli da me proposti per l'antologia garzantiana, in sostituzione di Trilogia della e e cioè: L'omo segno [...], L'Arca e Ombre sui veri [...] anche Segre preferisce, come noi due, Ombre sui veri». 


\section{Willy Schwarz}

Calzavara cercò informazioni presso il musicista Schwarz per musicare la cantata «Ritorno de Orfeo» (vedi Mario Baratto, Alfredo De Palchi, Ludovico Zorzi).

1. W.S., 15 giugno 1976, lett. f. ms. sf.

«Non sono riuscito a reperire passi di Steiner su Orfeo. ${ }^{90} \mathrm{Nep}$ pure ha potuto indicarmeli, per Lei, il Rag. Titobello. Il quale ha però avuto la gentilezza di copiare per Lei l'accluso brano di Schuré». ${ }^{91}$

2. E.C., 24 giugno 1976, lett. copia c. c. vel. ms.

«Mi dispiace che nelle opere di Steiner non si sia potuto trovare qualche cosa sull'argomento, poiché era proprio il pensiero di Steiner quello che desideravo conoscere».

\section{Cesare Segre}

Cesare Segre fu una delle guide di Calzavara. Il 25 ottobre 1967 Segre scrisse l'articolo «Due nuove figure nella letteratura dialettale contemporanea. Sospesi tra lingua e dialetto» per La Fiera letteraria. In seguito, e con lui, Calzavara scelse le poesie che compaiono nell'Almanacco dello Specchio del 1978 (vedi Marco Forti), in cui Segre introdusse la sezione dedicata al poeta. Nel 1980 Calzavara pubblicò in Tabula le poesie inedite «Chiccus» e «Vis grata puellae» (pp. 61-5), per le quali Segre scrisse la nota introduttiva (Calzavara 1980b). La scelta delle poesie che formano le raccolte poetiche di Calzavara fu compiuta con la consulenza di Segre, che scrisse le prefazioni a Come se nel 1974, a Ombre sui veri nel 1989 fino all'ultima opera dello scrittore, Rio terrà dei pensieri nel 1996. La decisione del titolo Ombre sui veri fu presa in tre, da Calzavara, Segre e Scheiwiller (perché il volume sarebbe dovuto uscire in coedizione con le edizioni All'Insegna del Pesce d'Oro, per cui vedi Vanni Scheiwiller, lettera nr. 30, e qui la lettera nr. 34), e Segre stesso si prese la responsabilità per la buona riuscita del lavoro (lettera nr. 33): Calzavara non smise mai di esternargli il suo riconoscimento (lettera nr. 23). [fig. 19]

91 Schuré 1889 
1. C.S., Milano, 26 febbraio 1967, lett. copia c. c. vel. datt.

«Lei ha saputo, senza snaturare la nostra migliore tradizione in dialetto (il mio caro Giotti...) portarvi i temi attuali, persino sociali».

2. C.S., 3 marzo 1967, lett. copia c. c. vel. datt.

3. E.C., Milano, 3 dicembre 1969, lett. copia c. c. vel. ms.

«È sfumata per me la speranza di essere pubblicato da Mondadori».92

4. C.S., Milano, 15 ottobre 1972, lett. f. ms. sf. [fig. 20]

«La rivista "Nuova Presenza" farà un numero dedicato a 4 o 5 poeti italiani. Ho suggerito che tra questi ci sia Lei. Si tratterebbe di pubblicare una dozzina di poesie, con mia introduzione; l'estratto verrebbe poi diffuso come volumetto. Non è proprio quello che Lei desiderava, ma un modo di rientrare in circolazione sì. ${ }^{93}$

5. E.C., Milano, 18 ottobre 1972, lett. copia c. c. vel. ms.

6. E.C., Milano, 18 novembre 1972, lett. f. ms. sf.

«Dopo il Suo scritto sui miei versi per Nuova Presenza il ricordo della recente rilettura d'un passo di Gustave Cohen dai Souvenirs d'Anphitéàtre mi ritorna alla mente».

7. E.C., Milano, 1 giugno 1973, lett. f. datt.

8. E.C., Milano, 22 marzo 1975, lett. copia c. c. vel. ms.

9. E.C., Milano, 4 aprile 1975, lett. copia c. c. vel. ms.

«Le mando questa volta qualche cosa di diverso, non so se meglio o peggio del solito: El menabò, scritto a Treviso la settimana scorsa».

10. E.C., Milano, 9 dicembre 1975, lett. vel. ms. sf.

11. E.C., Milano, 16 giugno 1977, lett. copia c. c. vel. ms.

«In relazione alla nostra conversazione di ieri sera sul Contenuto, ti mando, per il caso che tu non le avessi già e ti fossero utili, fotocopie di alcuni miei appunti inediti frammentari, che ho utilizzato in qualche occasione: 1) Confidenze agli amici per l'uscita di Come se. Infralogie ${ }^{94}$ 2) Poesia di Calzavara (I) 3) Poesia di Calza-

92 Si riferisce al rifiuto per l’Almanacco dello Specchio, per cui vedi Marco Forti.

93 Non risulta pubblicato.

94 Le pagine - riviste e aggiornate - uscirono per la prima volta in Calzavara 1996, 37-43. Cf. anche Calzavara 2017a, 26-7. 
vara II. Questo materiale mi è servito in parte a suo tempo per un breve articolo sul settimanale 7 giorni in Veneto del 18/9/75 N. 37 (Presenza di Calzavara) pubblicato senza firma». ${ }^{95}$

12. E.C., Milano, 26 novembre 1977, lett. copia c. c. vel. ms.

13. E.C., Milano, 29 gennaio 1978, lett. copia c. c. vel. ms.

14. E.C., Milano, 21 febbraio 1978, lett. copia c. c. vel. ms.

15.E.C., Milano, 30 novembre 1979, lett. vel. ms. sf.

16. E.C., Milano, 26 febbraio 1980, lett. copia c. c. vel. ms.

«Ieri proprio alla vigilia di fare una scappata a Treviso, mi hanno telefonato da Genova per invitarmi a una riunione di poeti tra il 21 e il 25 Aprile. ${ }^{96}$ Credo che la proposta sia partita soprattutto per tua indicazione e te ne sono molto grato».

17. E.C., Milano, 30 aprile 1980, lett. copia c. c. vel. ms.

18. E.C., Milano, 1 luglio 1982, lett. copia c. c. vel. ms.

19. E.C., Milano, 3 novembre 1982, lett. copia c. c. vel. ms.

20. E.C., Milano, 1 gennaio 1983, lett. copia c. c. vel. ms.

21. E.C., Milano, 1 marzo 1983, lett. copia c. c. vel. ms.

«Sulla proposta tua e di Ávila, mi sono provato a tradurre in veneto Guillen [...]. ${ }^{97}$ Bene o male non è stata però una cosa facile».

22.E.C., Milano, 3 aprile 1983, lett. copia c. c. vel. datt. e ms. sf. mt. 23. E.C., Milano, 30 aprile 1984, lett. copia c. c. vel. ms.

«Ti sono infinitamente grato del tuo aiuto».

24. E.C., Milano, 25 ottobre 1984, lett. copia c. c. 2 vell. datts.

«Lo scorso Agosto, a Vetriolo, quando meno me lo aspettavo, mi è venuto fuori un gruppetto di composizioni senza pretese [...]. Per quanto invece riguarda l'Antologia [...] ho capito anch'io, come tu mi avvertisti dopo, che sia meglio non inserire niente delle mie tre prime plaquettes, salvo conservare quale motto per

95 «Poesia. Presenza di Calzavara». Sette Giorni nel Veneto, 18 settembre 1975, 36-7.

96 Fernanda Pivano, «Autori italiani e americani per un convegno a Palazzo Doria Spinola. Genova-New York, via poesia». Corriere della Sera, 27 aprile 1980, 3.

97 Pablo Luis Ávila, a cui Calzavara scriveva il 2 aprile 1983: «Spero che le mie traduzioni siano accettabili. Ho fatto del mio meglio». Le poesie allegate sono «Albaro de istà», «Quei che no xe mai queti», «2 de novembre», «El viagio». Le traduzioni scelte furono «Quei che no xe mai queti» e «Albaro de istà», uscite con la «traduzione in trevigiano di Ernesto Calzavara», in Ávila 1983, 103-4. Il volume sarà presentato da Franco Fortini e Cesare Segre il 7 giugno 1985 a Milano. 
l'Antologia, i quattro versi eponimi dopo il frontespizio de Il tempo non passa».

25.E.C., Milano, 26 ottobre 1984, lett. copia c. c. vel. datt.

26. E.C., [ottobre 1984], lett. copia c. c. vel. ms.

27. E.C., Milano, 10 dicembre 1984, lett. copia c. c. vel. datt.

28. E.C., Milano, 18 marzo 1985, lett. copia c. c. 2 vell. datts.

«Tanto più che questa antologia sarà forse l'ultima raccolta di poesia che pubblicherò, oramai».

29. E.C., Milano, 19 maggio 1985, lett. copia c. c. vel. ms.

«Ti mando come d'accordo il gruppo delle mie sei poesie dell'anno scorso (Gita nei palui delle basse - Dona Litania - Xe tardi - Gli alberi - Destini - La me piera) delle quali però non ho proposto nessuna, per ora, per la Trilogia dell'e».

30.E.C., Milano, 1 giugno 1985, lett. copia c. c. vel. ms.

31. E.C., Milano, 16 maggio 1987, lett. copia c. c. 2 vell. mss.

«Ti mando queste sei poesie (Notte - Stagion d'istà - Ormai - Analisi - San Simplician - Yeti) pregandoti di leggerle quando avrai voglia e tempo».

32.E.C., Milano, 18 febbraio 1988, lett. copia c. c. vel. ms.

33. C.S., Pavia, 26 febbraio 1988, lett. f. datt.

«Quanto al volume, puoi stare certo che in qualunque situazione, in qualunque evenienza me ne considero responsabile».

34.E.C., Milano, 18 maggio 1988, lett. copia c. c. f. datt.

«Ti mando qui accluso per un esame generale, quanto occorre per il completamento della Trilogia della $e$ che pubblicherà Garzanti in coedizione con Scheiwiller e cioè: 1) L'indice generale di tutte le poesie già scelte e raccolte [...]. 2) Le poesie da me scelte non incluse nel manoscritto [...]. 3) n. 16 poesie nuove inedite [...]. 4) Testimonianze critiche [...]. Questa antologia dovrebbe essere la più importante indicazione panoramica di tutta la mia opera e della sua evoluzione attraverso decenni di ricerche laboriose e sofferte, tentativi e tentazioni forse non del tutto inescusabili».

35.C.S., Pavia, 10 luglio 1989, lett. f. ms. sf. [fig. 21]

36.E.C., Milano, 16 agosto 1989, lett. copia c. c. vel. ms. 
37. E.C., Milano, 7 gennaio 1990, lett. copia c. c. vel. datt.

38. E.C., [febbraio 1990], lett. f. ms. sf. mt.

39. E.C., Milano, 30 aprile 1990, lett. copia c. c. vel. ms.

40.E.C., Milano, 24 febbraio 1991, lett. copia c. c. vel. ms.

41. E.C., Milano, 7 ottobre 1991, lett. f. ms. fotoc.

\section{Mario Soldati}

Lo scrittore ringraziò Calzavara per l'invio di e. Parole mate Parole pòvare.

1. M.S., Milano, 22 febbraio 1967, lett. copia c. c. datt. vel. datt.

«Ricevo due copie, una da Lei e una da Vanni, delle Sue poesie che ho letto subito con vero trasporto».

\section{Giacinto Spagnoletti}

Spagnoletti scrisse l'articolo apparso il 21 marzo 1990 su Italiaoggi dal titolo «Protagonisti. Ernesto Calzavara, Maria Luisa Spaziani e Dario Bellezza. Le tre generazioni a confronto: come si è rinnovata la poesia in Italia»; lo stesso articolo uscì nella stessa data anche su Il Messaggero, con il titolo di poco diverso «Poesia. Calzavara, Spaziani e Bellezza a confronto. Tre campioni di eresia» (lettera nr. 4).

1. E.C., Milano, 22 febbraio 1990, lett. copia c. c. vel. ms.

2. G.S., Roma, 9 marzo 1990, lett. f. datt.

«Il suo libro rappresenta un grande passo in avanti della nostra poesia contemporanea».

3. E.C., Milano, 18 marzo 1990, lett. copia c. c. vel. ms.

4. G.S., Roma, 21 marzo 1990, lett. f. ms sf.

«Giacché è improbabile che lei veda presto quest'articolo apparso oggi sul Messaggero, ho piacere di spedirglielo».

5. E.C., Milano, 26 marzo 1990, lett. copia c. c. vel. ms.

«Debbo dirle che Le sono gratissimo di aver capito come pochi, quello che, fra gli altri problemi, è stato per me, in poesia il continuo dramma del linguaggio, della sua ricerca, della sua carica, delle sue scelte, del suo divenire e così pure il dramma della parola allo stato nascente [...]. Sono stato molto contento di quello che Lei 
mi ha scritto perché attendevo da tempo che si arrivasse a questa vera comprensione e così come Lei l’ha espressa».

\section{Adriano Spatola}

Nel 1969 Adriano Spatola scrisse il saggio Verso la poesia totale definendo il fenomeno dell'avanguardia poetica di quegli anni (Spatola 1969). Fondamento della sua teoria è la forte tensione verso un linguaggio universale, basato sulla poesia dell'immagine e sulla poesia fatta immagine come segnale visivo, le cui radici sono indicate nei pittogrammi primitivi, negli ideogrammi, nei grafismi infantili, nei giochi tipografici e concettuali di Mallarmé, nelle avanguardie del Futurismo e del Surrealismo. In quel periodo più poeti iniziarono, indipendentemente l'uno dall'altro, a sperimentare nuove forme di fare poesia, fra questi Calzavara con il suo Cembalo scrivano del 1977 (vedi la scheda su Gregorio Scalise).

1. E.C., Milano, 20 febbraio 1979, lett. copia c. c. vel. ms.

«Come d'accordo, Le mando un'altra, anzi due altre copie del mio Cembalo scrivano. Tenga presente in ogni modo, se, come gentilmente mi ha promesso, scriverà qualche cosa sul mio libretto, che esso dovrà comparire un giorno in veste tipografica migliore di quella che disgraziatamente ha avuto, fra l'altro con titoli in veneto dei vari dattilogrammi che non figureranno sopra il significato ma in altra pagina».

\section{Giovanni Tesio}

Insieme a Mario Chiesa scrisse il volume Il dialetto da lingua della realtà a lingua della poesia. Da Porta e Belli a Pasolini (Chiesa, Tesio 1978, su Calzavara in part. 2-7, 212-15, 220-1; lettera nr. 2). Il 10 novembre 1979 uscì l'articolo di Tesio «Le poesie di Ernesto Calzavara. Orfeo si perde a Manhattan» apparso su Tuttolibri (p. 11, lettera nr. 3). Sono del 1984 i due tomi de Le parole di legno, anch'essi in collaborazione con Mario Chiesa (alla cui scheda si rimanda; qui lettera nr. 11). Nel 1986 uscì «Ascoltando quel che dicono gli autori, si può disegnare una mappa della poesia dialettale. Come chiamarli: country o innovativi?», per la Rubrica "il nuovo Raccoglitore", sulla Gazzetta di Parma (89, 3 dicembre 1986, lettere nrr. 8-10).

1. E.C., Milano, 18 aprile 1978, copia c. c. 2 vell. datts.

2. G.T., 25 aprile 1978, lett. f. ms. sf. 
«La ringrazio veramente di cuore [...] delle parole troppo gentili, troppo generose che ha voluto indirizzarci sulla nostra impresa».

3. E.C., Milano, 16 novembre 1979, lett. copia c. c. vel. ms.

«Sono veramente molto grato della Sua recensione su Tuttolibri, specialmente per alcuni punti da me particolarmente graditi».

4. E.C., dicembre 1980, lett. f. ms. sf.

5. G.T., Torino, 7 gennaio 1981, lett. f. ms. sf.

6. G.T., Torino, 19 ottobre 1984, lett. f. ms. sf.

«Poiché io e Chiesa dovremmo essere direttamente implicati nell'organizzazione scientifica di un congresso sui poeti dialettali del Novecento e la cultura europea, ho deciso di cogliere l'occasione per scrivere un saggio [...]. Ho bisogno per questo di parlare con te».

7. E.C., Milano, 28 ottobre 1984, lett. copia c. c. vel. ms.

8. G.T., Torino, 21 aprile 1986, lett. f. datt.

«Ti trascrivo qui due o tre domande perché sto preparando un servizio sulla poesia in dialetto per il "Raccoglitore". Eccoti le domande: 1. Quando hai cominciato a scrivere in dialetto hanno agito su di te ragioni, per così dire, endogene, o anche ragioni sociologiche, ideologiche?».

9. E.C., Milano, 3 maggio 1986, lett. copia c. c. 2 vell. datts.

«1) Quando ho incominciato a scrivere in dialetto, hanno agito in me prevalentemente ragioni interne, endogene, come tu scrivi, che riempivano lo spazio dei sentimenti, degli impulsi fondamentali. Quelle sociologiche e ideologiche possono avere aperto forse senza accorgersene, qualche raro spiraglio, ma molto alla lontana, di riflesso».

10. G.T., Torino, 24 dicembre 1986, lett. f. ms. sf.

«Ti mando finalmente la cosetta che è venuta fuori dall'inchiesta [...] in cui ti ho coinvolto».

11.E.C., Milano, 9 gennaio 1987, lett. f. datt.

«Essa chiarisce e integra anche la situazione che tu e Chiesa avevate precisato nella prefazione a Parole di legno e meriterebbe una estensione maggiore». 


\section{Diego Valeri}

Lo scrittore commentò la raccolta e Parole mate Parole pòvare.

1. D.V., Venezia, 14 febbraio 1967, lett. copia c. c. vel. datt. «Mi pare che la Sua poesia sia giunta alla sua piena maturità».

\section{Angelo Zanon dal Bo}

Zanon Dal Bo informò Calzavara del seminario di poesia che Giovanni Pozzi stava organizzando presso l'Università di Friburgo (vedi Giovanni Pozzi; qui lettera nr. 5). Egli fu il tramite fra Calzavara e le varie attività che si svolgevano nella Svizzera italiana.

1. A.Z.d.B., Sonvico, 23 gennaio 1976, lett. f. datt.

«Quello che dici vale e come lo dici incanta».

2. E.C., Milano, 25 gennaio 1976, lett. copia c. c. vel. ms.

«È una poesia molto naturale e tu potresti farne una raccolta più ampia da pubblicare».

3. A.Z.d.B., Sonvico, 16 ottobre 1976, lett. 2 ff. mss. sf.

«Ho letto il tuo Come se e ne sono rimasto ammirato, dico ammirato per l'essenzialità poetica delle tue infralogie».

4. A.Z.d.B., Sonvico, 27 ottobre 1977, lett. f. datt.

«Ho riletto $E$ e Come se [...]. Arrivato a quelle che cominciano colle donne di Tombuctu, solo dopo un po' mi sono reso conto che sono in italiano. La tua sostanziosa personalità poetica ha conquistato la libertà del mezzo. [...] Il mezzo è il messaggio».

5. A.Z.d.B., Sonvico, 25 gennaio 1978, lett. f. datt.

«Ho avuto qualche notizia sul seminario indetto da Padre Pozzi. Presi in esame saranno sei poeti italiani. Tre in lingua: Pagliarani, Antonio Porta, Zanzotto. Tre in dialetto: tu, Biagio Marin e Loi».

6. A.Z.d.B., Sonvico, 9 dicembre 1979, lett. f. datt. 
«Ho ascoltato iersera alla RSI la intervista che ti ha fatto Brevini per il Diario Culturale [...]. ${ }^{98}$ Non è stata trasmessa la lettura della seconda poesia, quella che doveva esemplificare il tuo sperimentalismo».

7. E.C., Milano, 20 dicembre 1979, lett. copia c. c. vel. ms.

8. E.C., Milano, 16 aprile 1980, lett. copia c. c. vel. ms.

«Ho ricevuto finalmente l'analisi strutturale della mia poesia Tutti i mati fa i so ati, tratta dal Come se, composta dalla Foletti». ${ }^{99}$

9. A.Z.d.B., Sonvico, 21 aprile 1980, lett. f. ms. sf.

«Mi sono informato presso il redattore [...] del Corriere del Ticino: Cusatelli gli ha promesso un articolo su di te, ma non lo ha ancora mandato». ${ }^{100}$

10. E.C., 23 aprile 1980, lett. copia c. c. vel. ms.

11.A.Z.d.B., Sonvico, 17 gennaio 1983, lett. f. datt.

12.E.C., Milano, 19 marzo 1991, lett. f. ms. sf.

\section{Sandro Zanotto}

Sandro Zanotto fu caro amico di Calzavara, oltre a essere stato tra i primi a considerare il recupero del dialetto veneto. Su L'Avvenire d'Italia pubblicò l'articolo «E di Ernesto Calzavara» il 12 luglio 1967, e nel luglio 1975 l'articolo su Venetonotizie «Recupero letterario dei dialetti veneti. Con la stessa dignità e grandezza di quello in lingua e con in più una capacità di incidenza nella realtà sociale e umana della regione». Su Il Gazzettino il 12 ottobre 1979 uscì «Poesie di Ernesto Calzavara. Analfabeto», e il 19 gennaio 1984 la recensione a Le parole di legno, di Mario Chiesa e Giovanni Tesio: «Un'antologia della poesia italiana del '900 in dialetto. Quelle parole di legno» (vedi Mario Chiesa, Giovanni Tesio) [fig. 22].

1. S.Z., Padova, 16 ottobre 1966, lett. f. ms. sf.

«Scheiwiller [...] è senz'altro d'accordo su Pasolini e farà di tutto per farlo aderire, il che va molto bene. Egli è poi della mia opinione

98 Radio della Svizzera Italiana, 8 dicembre 1979, 1 f. datt.

99 Vedi la scheda su Giovanni Pozzi.

100 Vedi la scheda su Giorgio Cusatelli. 
sul fatto che sia necessario invitare anche Zanzotto». ${ }^{101}$ [figg. 23-25]

2. E.C., Milano, 27 ottobre 1966, lett. copia c. c. f. ms.

«Mi tengo in contatto ogni tanto con Scheiwiller il quale però non ha ancora potuto incontrarsi con Pasolini».

3. E.C., Milano, 18 dicembre 1977, lett. copia c. c. 2 vell. mss.

4. S.Z., Treviso, 8 febbraio 1990, lett. f. ms. sf.

\section{Antonio Zanussi}

Antonio Zanussi ebbe l'incarico di seguire i lavori per l'incisione della 'e' sul sasso del giardino di casa Calzavara (si veda a scheda su Adriano Bellotto).

1. E.C., Milano, 30 giugno 1975, lett. copia c. c. vel. datt.

«Poiché Lei gentilmente si è offerto di farmi l'ingrandimento della lettera $e$ per il marmista, tratta da quell'alfabeto arcaico latino che ebbi a mostrarLe, le mando oltre che la fotocopia di questo, anche fotocopia dell'ingrandimento che sono riuscito a farmi ottenere. Sotto certi aspetti esso è abbastanza interessante, come vedrà, perché nell'operazione è saltata fuori inaspettatamente una lettera e di una sorprendente primitività e magicità quasi picassiane. Ma temo che scolpita in quel sasso non venga fuori bene o ne sia difficile la riproduzione con la stessa evidenza e plasticità, anche per motivi tecnici. Quindi è necessario, sempre sulla stessa falsariga, farne un esemplare regolare e normale (eventualmente anche un altro un po' grezzo). Dal confronto, così si potrà vedere meglio (anche sentito lo scalpellino) quale convenga di più riprodurre di questi prototipi emblematici dei rapporti universali. Forse in rapporto alla misura del sasso, si potrebbe ingrandire leggermente il modello di un centimetro o due».

2. A.Z., Treviso, 3 luglio 1975, lett. f. datt.

«Ho ricevuto l'ingrandimento della lettera $\mathrm{E}$ in forma primitiva direi arcaica. Cercherò di ingrandirla di quel poco che Lei desidera, ma è importante sapere per me, come dovrebbe essere la incisione nel sasso, se con una punta, rozza e ineguale come dovrebbe riuscire adoperando questo semplice strumento».

101 Si tratta dell'istituzione di un nuovo premio letterario, denominato «La barchessa», da tenersi a Levico, per cui si rimanda alla scheda su Pier Paolo Pasolini. 
3. A.Z., Treviso, 5 dicembre 1977, lett. f. datt.

4. E.C., Milano, 21 dicembre 1977, lett. copia c. c. vel. ms.

5. A.Z., Treviso, 14 gennaio 1978, lett. f. ms. sf.

6. E.C., Milano, 21 gennaio 1978, lett. copia c. c. vel. datt.

7. A.Z., Treviso, 8 marzo 1978, lett. f. datt.

8. A.Z., Treviso, 22 novembre 1978, lett. f. datt.

9. E.C., Milano, 7 dicembre 1978, lett. copia c. c. vel. datt.

10.A.Z., Treviso, 14 dicembre 1978, lett. f. datt.

\section{Ludovico Zorzi}

Nel 1979 Calzavara chiese informazioni allo studioso per musicare la cantata «Ritorno de Orfeo» che Calzavara aveva composto tre anni prima (vedi Mario Baratto, Alfredo de Palchi, Willy Schwarz).

1. L.Z., Firenze, 5 luglio 1978, lett. f. ms. sf.

2. E.C., Treviso, 13 agosto 1979, lett. copia c. c. 2 vell. mss.

«Ho pensato a Lei che conosce bene anche il teatro per chiederLe il Suo parere su un'idea che mi è venuta sin da quando in America composi il Ritorno de Orfeo [...]. Proporre cioè quella cantata come un testo da musicare o quanto meno da rappresentare o solo recitare o leggere per il pubblico di qualche ambiente più o meno teatrale o solo culturale. Lei che ne pensa? In caso positivo a chi potrei rivolgermi (magari a Suo nome)? Qualche anno fa scrissi a Ripa di Meana per la Manifestazione della Biennale di Venezia, ma non ebbi risposta. Mi rivolsi ad un noto musicista d'avanguardia di Firenze, G. Cardini [...]. Qualche vaga promessa di interessamento e poi nulla. Non ne parlai più a nessuno. Fra l'altro, infine, ho pensato che potesse interessare a qualche cineasta anche la trasposizione in film di gruppi delle mie poesie, un film naturalmente di fantasia, surreale ecc. Ma a chi far capo?».

3. L.Z., Firenze, 21 agosto 1979, lett. f. datt.

«Baratto è molto amico di Luigi Nono; e questa, secondo me, è la via diretta per avvicinare un importante musicista contemporaneo. Altri compositori moderni io non ne conosco; e così pure cineasti (ma l'idea del cinema mi sembra meno realizzabile, rispetto alla messa in musica di un brano lirico sotto la forma della cantata)». 


\section{Bibliografia}

\section{Testi di Calvazavara}

Calzavara, E. (1946). Il tempo non passa. Milano: Tip. Maserati.

Calzavara, E. (1947). I fiori di carta. Milano: Tip. Omnia.

Calzavara, E. (1948). Il nuovo mondo. Milano: Tip. Omnia.

Calzavara, E. (1960). Poesie dialettali. Treviso: Libreria Canova.

Calzavara, E. (1966). e. Parole mate Parole pòvare. Milano: All'Insegna del Pesce d'Oro.

Calzavara, E. (1971). «Poesia in dialetto e poetiche moderne. Note». Ateneo Veneto, 9, genn.-dic. 1971, 199-210.

Calzavara, E. (1974). Come se. Infralogie. Prefazione di C. Segre. Milano: All'Insegna del Pesce d'Oro.

Calzavara, E. (1977). Cembalo scrivano. Esercizi per dattilogrammi. Milano: All'Insegna del Pesce d'Oro.

Calzavara, E. (1978). «Katacuna e altre poesie». Introduzione di C. Segre. Almanacco dello specchio, 7, 159-61.

Calzavara, E. (1979). Analfabeto. Milano: Guanda.

Calzavara, E. (1980a). «Finzion», «Studio n. 4», «L'atto pubblico». Nuovi Argomenti, 67-68, 78-81.

Calzavara, E. (1980b). «Chiccus», «Vis grata puellae». Tabula, 3-4, 61-5.

Calzavara, E. (1981). «Poesia in dialetto e poetiche moderne. Note». Cortelazzo, M. (a cura di), Guida ai dialetti veneti, vol. 3. Padova: CLEUP, 119-50.

Calzavara, E. (1984). Le ave parole. Introduzione di S. Agosti. Milano: Garzanti.

Calzavara, E. (1989). Ombre sui veri. Poesie in lingua e in dialetto trevigiano (1946-1987). 1a ed. Introduzione di C. Segre. Milano: Garzanti.

Calzavara, E. (1990). Ombre sui veri. Poesie in lingua e dialetto trevigiano (19461987). 2 ed. Introduzione di C. Segre. Milano: Garzanti.

Calzavara, E. (1996). Rio terrà dei pensieri. A cura di M. Rizzante; prefazione di C. Segre. Milano: Scheiwiller.

Calzavara, E. (2001). Ombre sui veri. Poesie in lingua e dialetto trevigiano (19461987). Introduzione di C. Segre. Milano: Garzanti.

Calzavara, E. (2006). Poesie dialettali. 2a ed. Treviso: Canova edizioni.

Calzavara, E. (2017a). Raccolte poetiche 1, 1960-1974. Ed. critica commentata a cura di A. Rinaldin. Venezia: Edizioni Ca' Foscari. https://phaidra.cab. unipd.it/o:432253.

Calzavara, E. (2017b). Raccolte poetiche 2, 1979-1984. Ed. critica commentata a cura di V. Gobbato. Venezia: Edizioni Ca' Foscari. https://phaidra.cab. unipd.it/o:432254.

\section{Fonti}

Agosti, S. (1995). «La lingua di Calzavara». Poesia italiana contemporanea. Saggi e interventi. Milano: Garzanti, 113-17.

Armandi, G. (1974). «Gli sfoghi di Giovanni Comisso (Raccolte trecento lettere)». La Fiera letteraria, 17 novembre, 18-19.

Ávila, P.L. (1983). Sonreido va el sol. Poesie e studi offerti a Jorge Guillén. Milano: All'Insegna del Pesce d'Oro. 
Bonora, E. (1976). «Profilo della poesia dialettale. La poesia napoletana da Salvatore Di Giacomo a Eduardo de Filippo. Tessa, Giotti e altri». Storia della letteratura italiana. Torino: Petrini, 799-804.

Bonora, E. (1981). «Il dibattito sulla letteratura dialettale dall'età veristica ad oggi». Giornale Storico della Letteratura Italiana, 158, 481-517.

Bonora, E. (1984). «La condizione del poeta dialettale oggi». Giornale Storico della Letteratura Italiana, 161, 337-55.

Borsetto, L. (1979). «La poesia di Ernesto Calzavara tra lingua e dialetto». Medioevo e Rinascimento Veneto con altristudi in onore di Lino Lazzarini. Vol. 2, Dal Cinquecento al Novecento. Padova: Antenore, 439-56.

Borsetto, L. (1980). Recensione di Calzavara 1979. Paragone, 360-362, febbraio-aprile, 185-8.

Borsetto, L. (1982). «Tra normalizzazione e sperimentazione: appunti sulla questione del verso». Baldassarri, G. (a cura di), "Quasi un picciolo mondo». Tentativi di codificazione del genere epico nel Cinquecento. Milano: Unicopli, 91-127.

Borsetto, L. (1984). «Dialetto, antidialetto, "deriva". La scrittura poetica degli anni Settanta». Mazzamuto, P. (a cura di), La letteratura dialettale in Italia. Dall'Unità ad oggi. Palermo: Università di Palermo, 829-50.

Borsetto, L. (1989a). Lingua dialetto poesia. Cinque analisi. Marin, Cergoly, Calzavara, Giacomini, Bartolini. Ravenna: Edizioni Essegi.

Borsetto, L. (1989b). «"Can-can, can-ton [...] can-icole”. Ernesto Calzavara tra lingua e dialetto». Borsetto 1989a, 69-90.

Borsetto, L. (1989c). «"Se domandâti, lune...". Emblemi dell'“io" e della natura nel canzoniere di Giacomini». Borsetto 1989a, 91-132.

Borsetto, L. (2007). «Il "bestiario" di Ernesto. Per un ritorno alla poesia di Calzavara». Tamiozzo Goldmann 2007, 49-71.

Brevini, F. (1990). Le parole perdute. Dialetti e poesia nel nostro secolo. Torino: Einaudi.

Calza, G.C. (1975). L'incanto sottile del dramma No. La principessa Aoi. Milano: Edizioni di Vanni Scheiwiller.

Chiesa, M. (1982). «Appunti sullo studio della poesia contemporanea in dialetto». Lettere Italiane, 34(1), 85-94.

Chiesa, M. (1984). «Per una mappa della poesia contemporanea in dialetto». Giornale Storico della Letteratura Italiana, 161, 356-84.

Chiesa, M.; Tesio, G. (a cura di) (1978). Il dialetto da lingua della realtà a lingua della poesia. Da Porta e Belli a Pasolini. Torino: Paravia.

Chiesa, M.; Tesio, G. (a cura di) (1984). Le parole di legno. Poesia in dialetto del '900 italiano. 2 voll. Milano: Mondadori.

Conte, G. (1983). L'Oceano e il Ragazzo. Milano: Rizzoli.

Cima, A. (1984). Ipotesi d'amore. Milano: Garzanti.

Comisso, G. (1972). Trecento lettere di Giovanni Comisso a Maria e Natale Mazzolà (1925-1968). Introduzione, commento e note a cura di E. Dematté. Treviso: Editrice trevigiana.

Della Corte, C. (1977). Cuor di padrone. Venezia: Edizioni del Ruzante.

Della Corte, C. (1979). «Poeti nel Veneto». Inchiesta sulla poesia. La poesia contemporanea nelle regioni d'Italia. Foggia: Bastogi Editrice Italiana, 97-9.

Della Corte, C. (1980). Grida dal palazzo d'inverno. Milano: Mondadori.

Di Meo, Ph.; Para, J.-B. (1983). «De l'autre côté des Alpes, un autre poésie». Europe, 649, 91-5. 
Fasolo, U.; Fiscon, A.; Rebellato, B. (a cura di) (1974). Presenza religiosa nella poesia contemporanea italiana. Premio Camposanpiero 1972. Introduzione di F. Ulivi. Padova: Rebellato.

Gobbato, V.; Uroda, S. (a cura di) (2014). "Una raffinata ragnatela»: Carlo della Corte tra letteratura e giornalismo nel secondo Novecento italiano. Venezia: Edizioni Ca' Foscari. http://doi.org/10.14277/978-88-97735-70-๑.

Grignani, M.A. (1985). Recensione di Calzavara 1984. Autografo, 4, febbraio, 97-100.

Haller, H.W. (1986). The Hidden Italy. A Bilingual Edition of Italian Dialect Poetry. Detroit: Wayne State University Press.

Haller, H.W. (1992). «Ernesto Calzavara. Ombre sui veri». L'anello che non tiene. Journal of Modern Italian Literature, 4, 1-2, spring-fall, 99-101.

Lorenzini, N. (1984). «La poesia imperfetta». Recensione di Calzavara 1984. Il Verri, 3-4, luglio-dicembre, 138-9.

Marin, B. (1970a). I canti dell'isola. 1912-1969. Trieste: Cassa di Risparmio di Trieste.

Marin, B. (1970b). La vita xe fiama. Poesie 1963-1969. A cura di C. Magris, prefazione di P.P. Pasolini. Torino: Einaudi.

Marin, B. (1981a). Nel silenzio più teso. Introduzione di C. Magris, scelta e note a cura di E. Serra, traduzione italiana a fronte di G.B. Pighi e E. Serra. Milano: Rizzoli.

Marin, B. (1981b). Poesie. A cura di C. Magris e E. Serra. Milano: Garzanti.

Mazzolà, N. (1960). Pietro aspetta il sole. Cronache partigiane. Roma: Tip. Farri.

Mazzolà, N.; Calzavara Mazzolà, M. (1968). Gino Scarpa. Colloqui con Arturo Martini. A cura di M. e N. Mazzolà, introduzione di G. Piovene. Milano: Rizzoli.

Orelli, G. (1986). Svizzera italiana. Brescia: La Scuola.

Pasolini, P.P. (a cura di) (1952). Poesia dialettale del Novecento. Con traduzioni a piè di pagina a cura di M. Dell'Arco e P.P. Pasolini. Parma: Guanda.

Pasolini, P.P. (a cura di) (1995). Poesia dialettale del Novecento. Prefazione di G. Tesio. Torino: Einaudi.

Pozzi, G. (1981). La parola dipinta. Milano: Adelphi.

Pozzi, G. (1992). «ll cantico di frate Sole di san Francesco». Asor Rosa, A. (a cura di), Letteratura italiana. Le opere, 1. Dalle origini al Cinquecento. Torino: Einaudi, Torino, 3-26.

Rinaldin, A. (2006). Gli strumenti del poeta. Notizie dal Fondo Calzavara. Roma; Padova: Editrice Antenore.

Rinaldin, A. (2020). «"Parole che risignificano". Materiali inediti dalle "Carte del Contemporaneo": Carlo Della Corte e Gianfranco Folena leggono Ernesto Calzavara». Santagata, E.; Zanato, T. (a cura di), Dieci brevi studi per Silvana Tamiozzo, con un inedito di Fabio Pusterla. Padova: libreriauniversitaria.it edizioni, 49-57.

Rizzante, M. (a cura di) (1993). «Per Ernesto Calzavara», num. monogr., Baldus, 1. Roqueta, M. (1974-75). Vèrd paradis. 2 voll. Tolosa: Institut d'estudis occitans.

Schuré, É. (1889). Les Grands Initiés. Esquisse de l'histoire secrète des religions: Rama, Krishna, Hermès, Moïse, Orphée, Pythagore, Platon, Jésus. Paris: Perrin.

Spatola, A. (1969). Verso la poesia totale. Salerno: Rumma editore.

Tamiozzo Goldmann, S. (2006). «ll laboratorio di Ernesto Calzavara». Rinaldin 2006, 7-13.

Tamiozzo Goldmann, S. (a cura di) (2007). Giornata di Studio su Ernesto Calzavara $=$ Atti della Giornata di Studi (Venezia, 9 giugno 2006). Ravenna: Angelo Longo Editore. 


\section{Appendice iconografica}

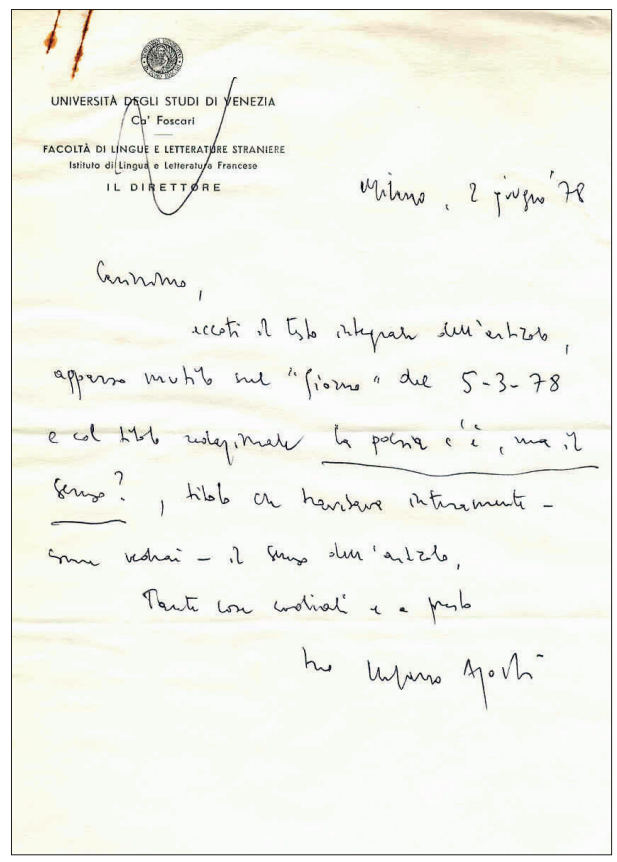

Figura 1

Lettera di Stefano Agosti

a Ernesto Calzavara.

Milano, 2 giugno 1978

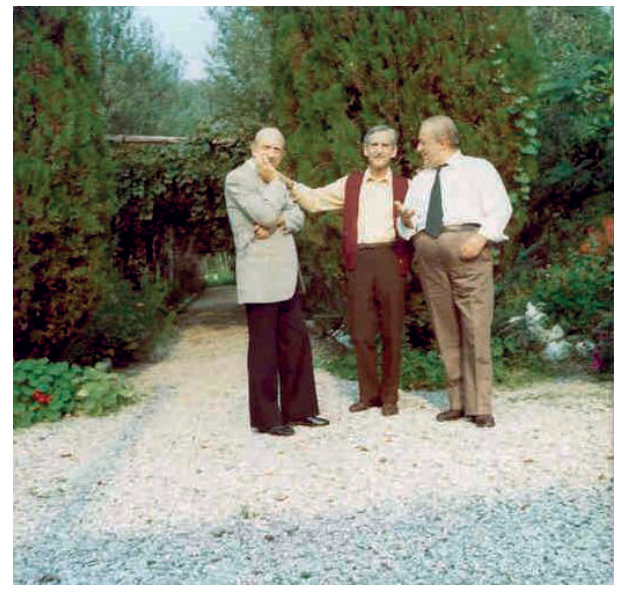

Figura 2

Giuseppe Mazzariol,

Ernesto Calzavara e Mario Baratto nella casa trevigiana del poeta (1975) 


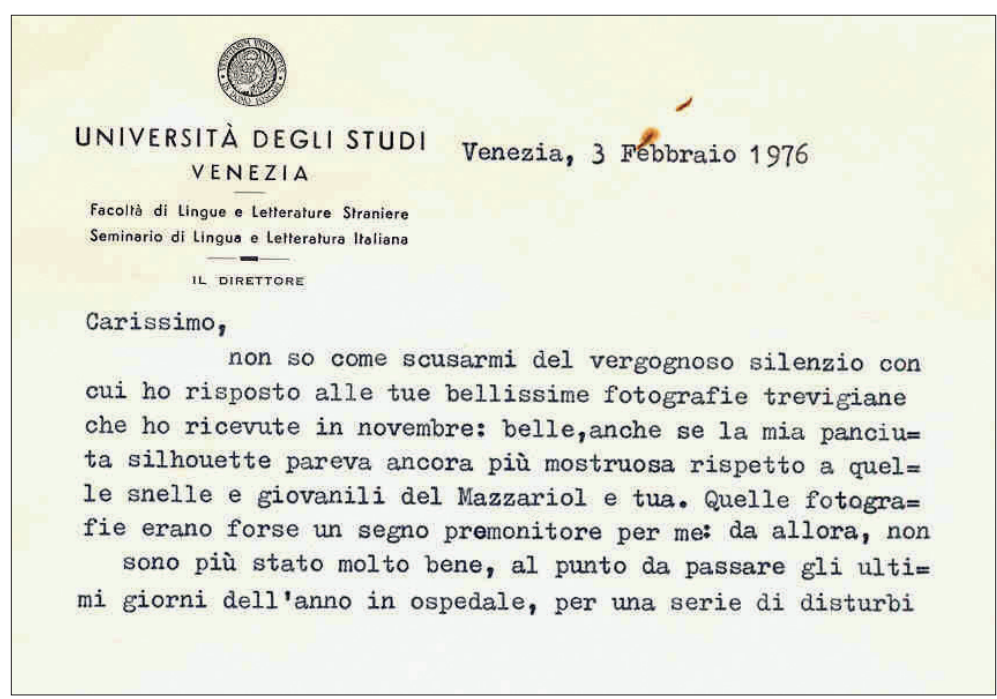

cardiocircolatori. Ora, sono da quindici giorni sottoposto a una dieta molto rigorosa, che dovrebbe, in prospettiva, dar= mi la linea "Mazzariol",e una maggior scioltezza a tutto il mio metabolismo (?). Ma non è facile tenere il ritmo, e qual= che volta sono preso da profonda depressione. Grazie ancora, per quelle splendide immagini di campagna tre= vigiana di primo autunno, e un augurio in ritardo ma affettuo= so per l'attività del nuovo anno. Tuo

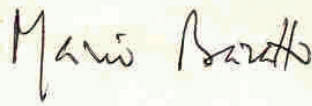

Figure 3-4 Lettera di Mario Baratto a Ernesto Calzavara. Venezia, 3 febbraio 1976 

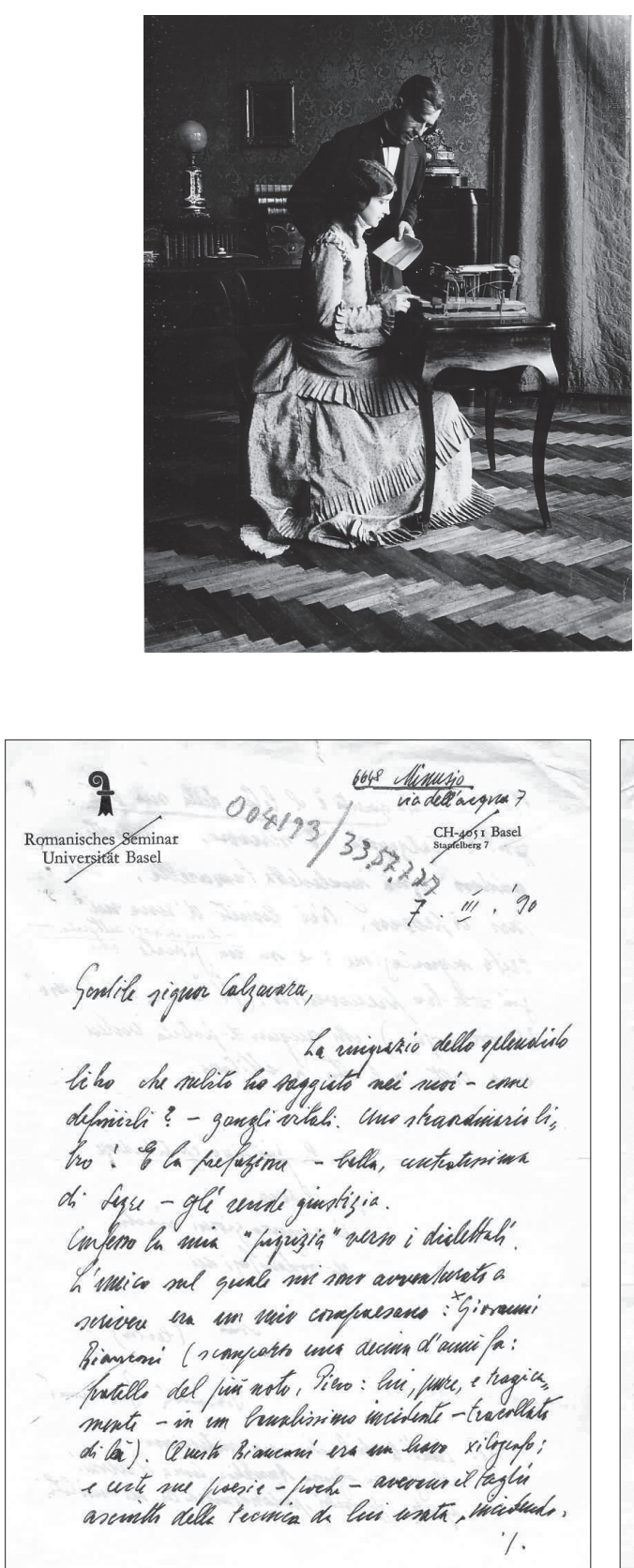

Vods, routh che quent i is likes dells men vita.

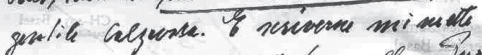

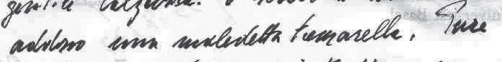
mi cifioness.' Nai essicite a'une nec bita jö ho presumestits. (Al Crevica del Tivino:

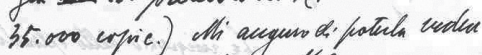
un inta o l'alts 1 elficoms.

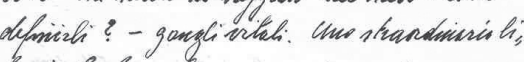

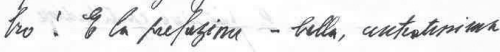
d. Lyu - gle sende giostijia. Conferso la mia "frizusis" quars i diclettsh"

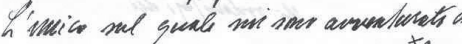

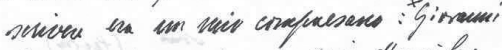

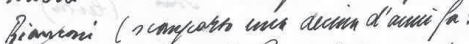

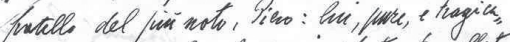
mente - in em homalisimo wecisente - tracoluts

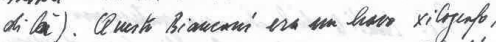
1 cuct sue fovie - forch - averour el logln

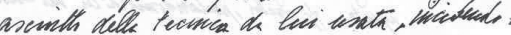

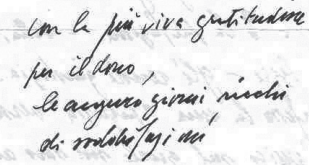

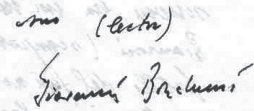

Figure 6-7 Lettera di Giovanni Bonalumi a Ernesto Calzavara. Minusio, 7 marzo 1990 


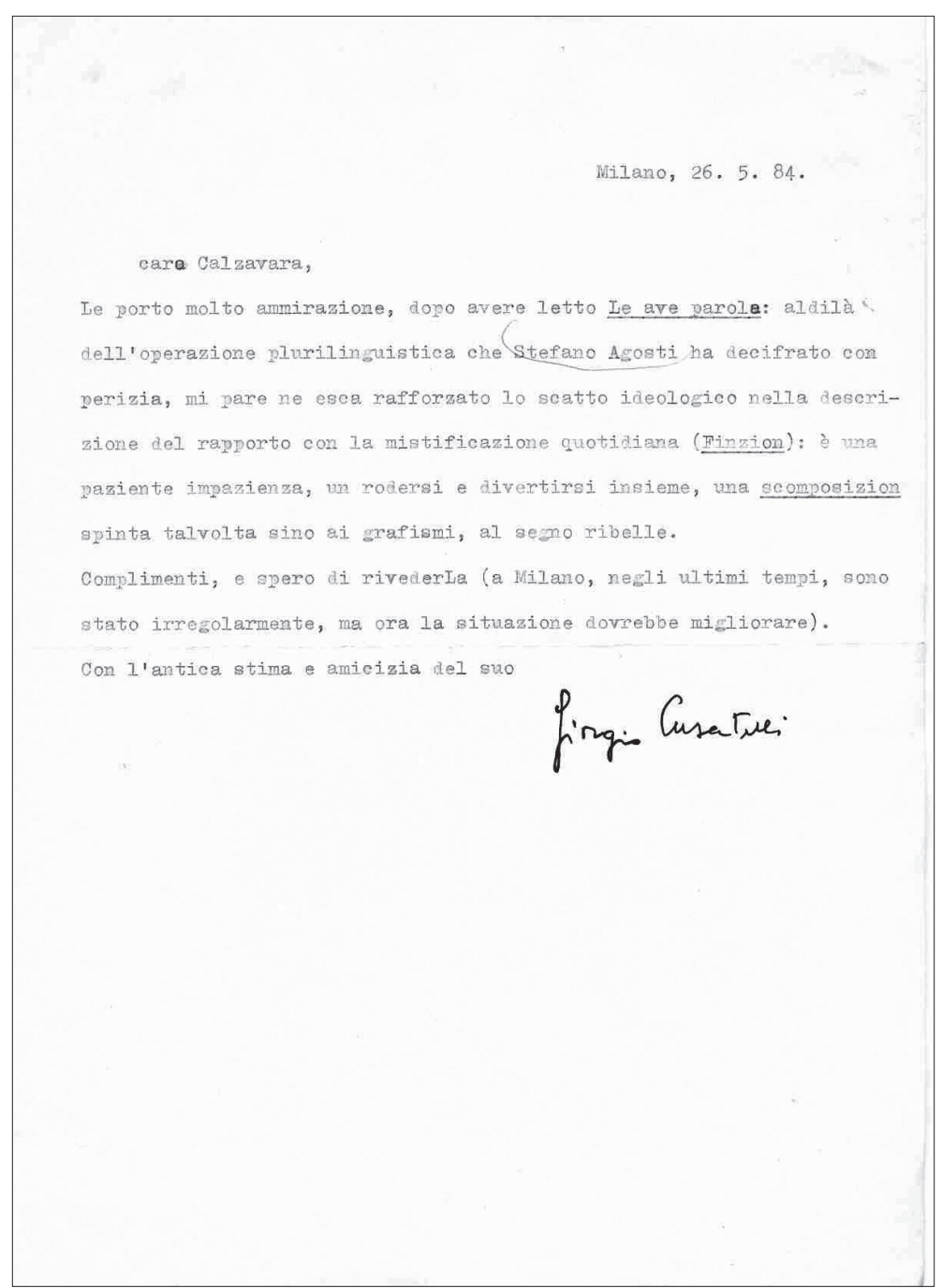

Figura 8 Lettera di Giorgio Cusatelli a Ernesto Calzavara. Milano, 26 maggio 1984 


\section{menta \\ rivista di confine \\ via s. emidio rosso, 9 \\ 63100 ascoli piceno}

Caro Calzevera,

quasi sicuramente la rassegna di

marka sui poeti dialettali sarà ristampata in vo-

lume (anzi, in due) da una casa editrice giovane

ma seria. Il lavoro sarà curato da me, con l'aiu-

todi Tiziana Alberti, della redazione. Ia mia idea

è di presentare almeno un panorama della produzione dialettale dell'ultimo ventennio, per cui, le sarei molto grato se volesse farmi conoscere le pubblicazioni che precedono Analfabeto.

Dovrebbe anche sistemare, in due-tre cartelle,

I'intervento di poetica che, grossomodo, covrisnonde al primo quesito del nostro formulario e preparare una scheda con la sua bibliografia critica completa.

Ciascun autore avrà uno spazio compatto: prima i testi poi la nota di poetica. In fondo al voliume, la scheda biobibliografica e quella critica. Lo scopo è quello di offrire uno sguardo non superficiale del lavoro testuale e teorico di ogni poeta.

Se la casa editrice opterà per i due tomi, lei sarà senza dubbio nel primo, E non solo per anzianità.

Con sincerità e modestia, posso dirle che per me è uno dei più bravi. Quindi bisognerà cercare di comporre quello 'spazio' in modo che presenti il meglio delle sue buone produzioni noetiche.

Contiamb di consegnare il materiale entro febbraio o marzo. Le darò comunque conferma della nuove pubblicazione appena possibile. Intanto la ringrazio della collaborazione già accordataci e le auguro, anche a nome della redazione, buone feste

affettuosi saluti

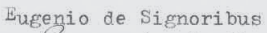

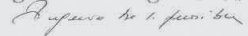

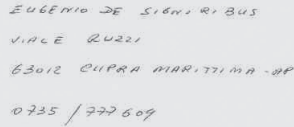

Figura 9 Lettera di Eugenio De Signoribus a Ernesto Calzavara. Ascoli Piceno, 15 dicembre 1982 
Anna Rinaldin

Rapporti culturali nella tradizione poetica del Novecento dalle lettere di Ernesto Calzavara
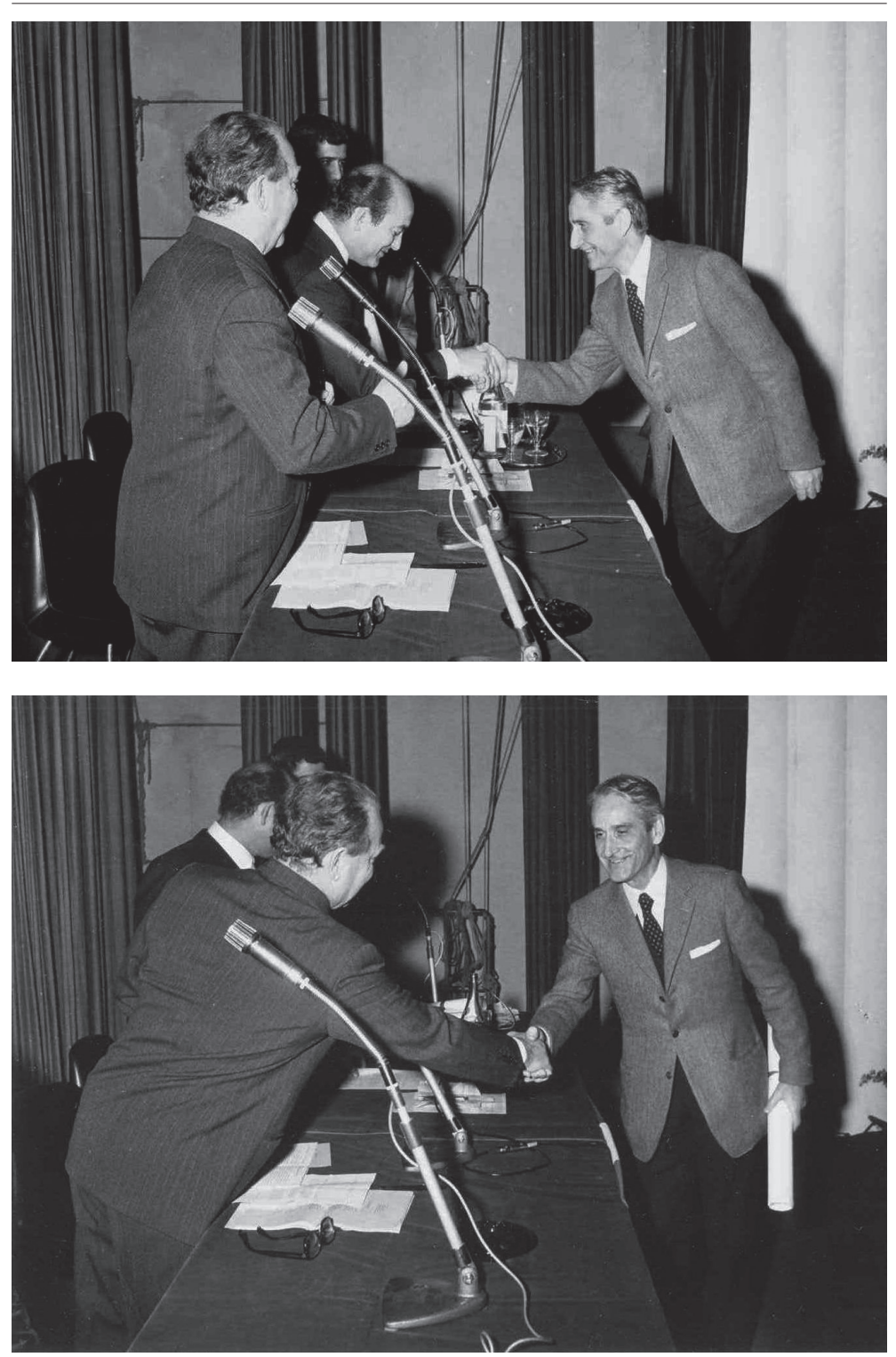

Figure 10-11 Calzavara scrive sul retro delle foto: «Premio Camposampiero 29/X/1972». Si riconoscono Ugo Fasolo, forse anche Armando Fiscon e Bino Rebellato, organizzatori del Premio 


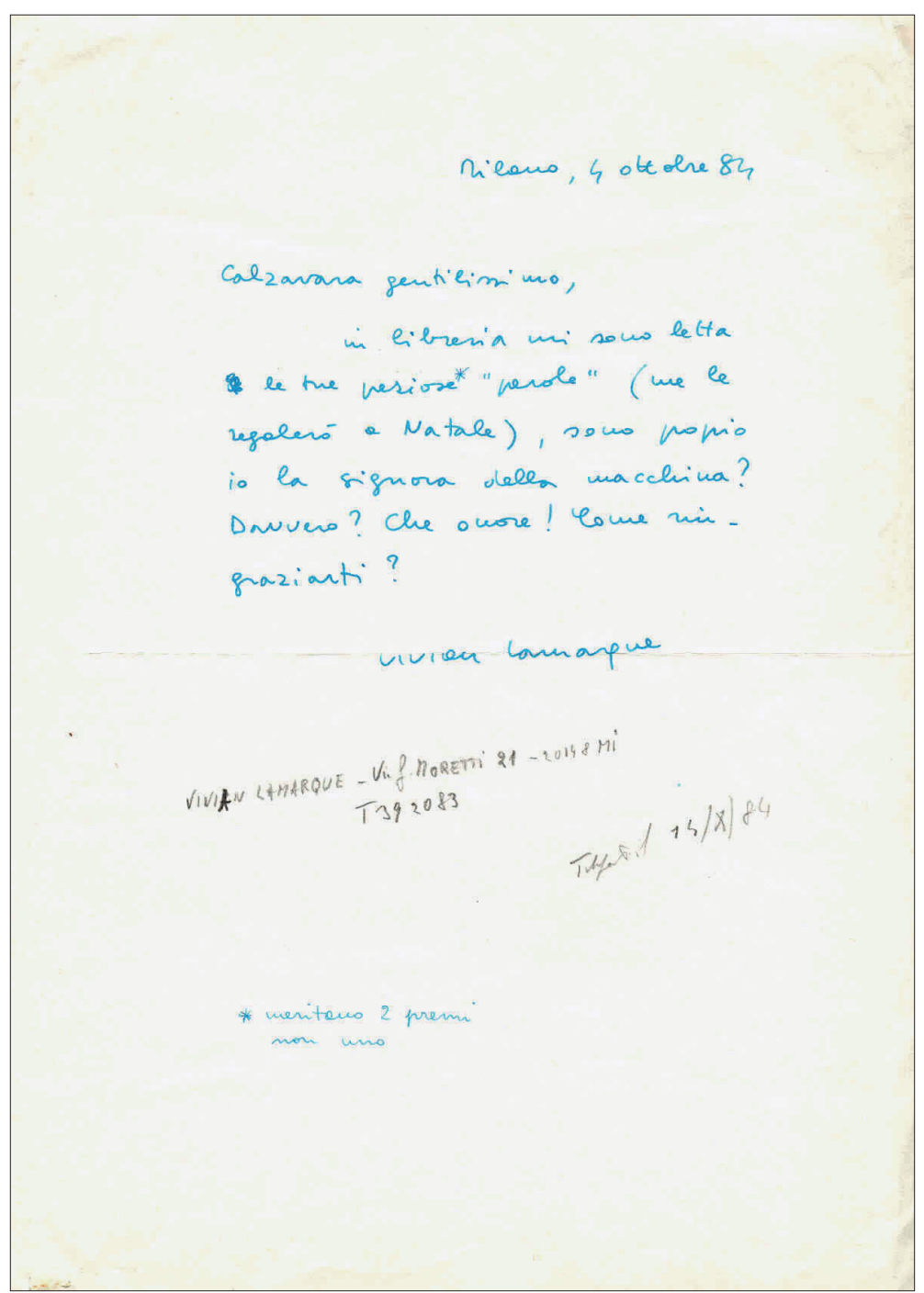

Figura 12 Lettera di Vivian Lamarque a Ernesto Calzavara. Milano, 4 ottobre 1984 


\section{curo colpawares.}

Grado $25 \times 1{ }^{\prime}$ be

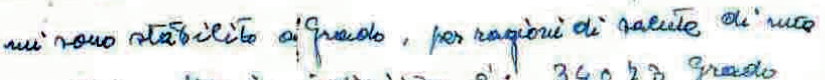

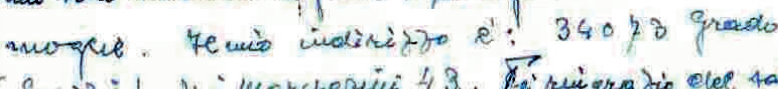

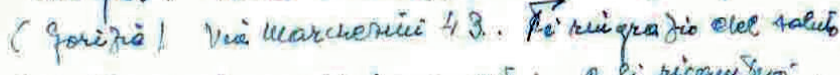

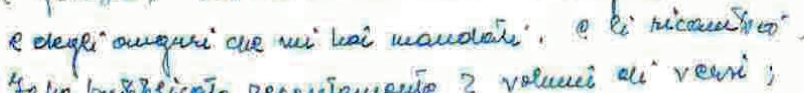

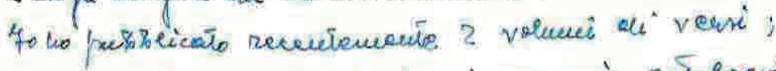

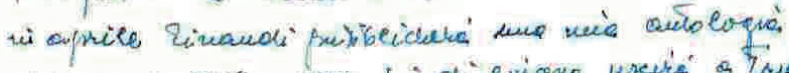

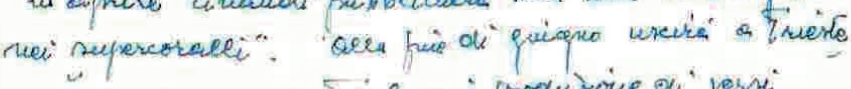

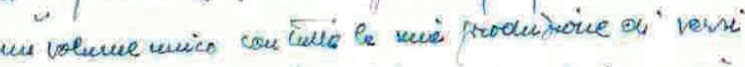

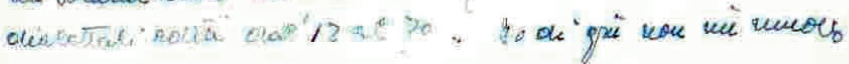
peis. oqui beve a te.

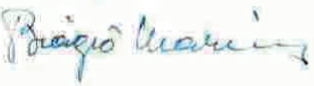

Figura 13 Lettera di Biagio Marin a Ernesto Calzavara. Grado, 25 dicembre 1969 


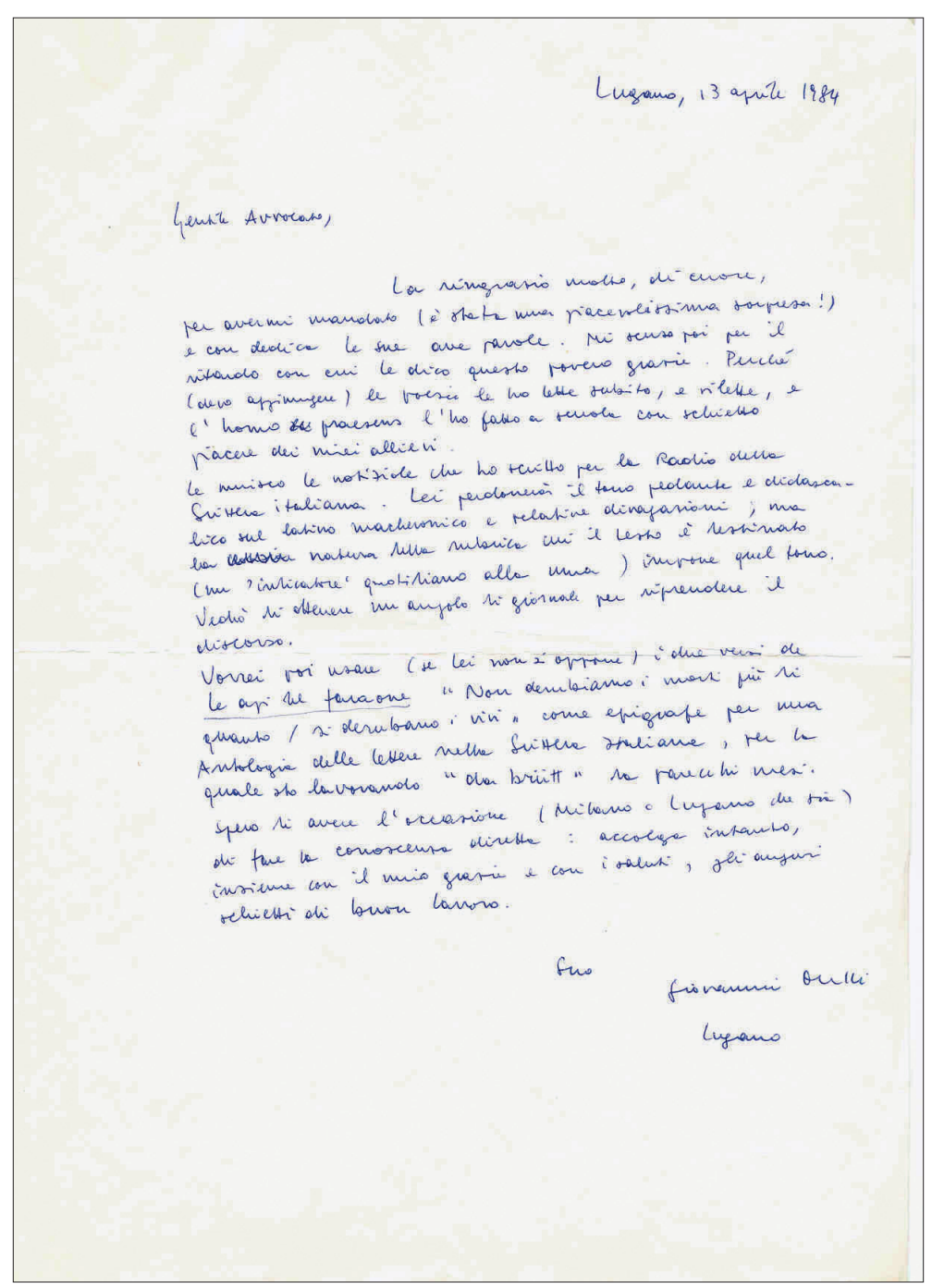

Figura 14 Lettera di Giovanni Orelli a Ernesto Calzavara. Lugano, 13 aprile 1984 
Anna Rinaldin

Rapporti culturali nella tradizione poetica del Novecento dalle lettere di Ernesto Calzavara

Giuseppe Ponliggia

20129 Milana - Via R. Forneti, ?

Milans, 27.11.1984

aro Calzavare,

wopaltutto del liens: legferb e cone

me curculure sicifuente, un viaffis dentio gli stroti del

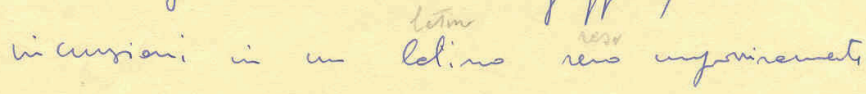
vive dalle contiguit: delle altre lingue.

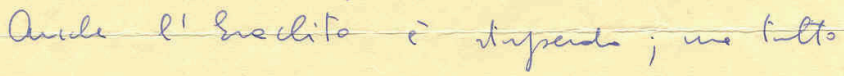
$l^{\prime}$ opere i ingere per $l^{\prime}$ antonits' e iniene le undilits del m toms: eamch ju le me unifonire seticuse, bu allevien;

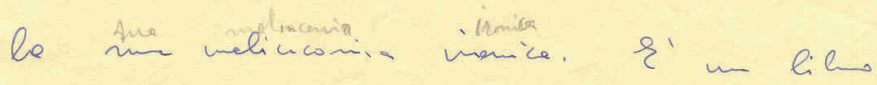

Figure 15 Lettera di Giuseppe Pontiggia a Ernesto Calzavara. Milano, 27 novembre 1984

163

Quaderni Veneti

e-ISSN 1724-188X 


$$
\begin{aligned}
& \text { bello e ne nows lieto pur te. } \\
& \text { A parto, yous. Ti wask i wiei argur } \\
& \text { di trun levons, il wi solets coro }
\end{aligned}
$$

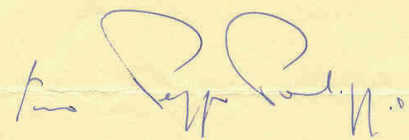

Figure 16 Lettera di Giuseppe Pontiggia a Ernesto Calzavara. Milano, 27 novembre 1984 
Figura 17 Lettera di Giovanni Pozzi a Ernesto Calzavara. Friburgo, 14 maggio 1978

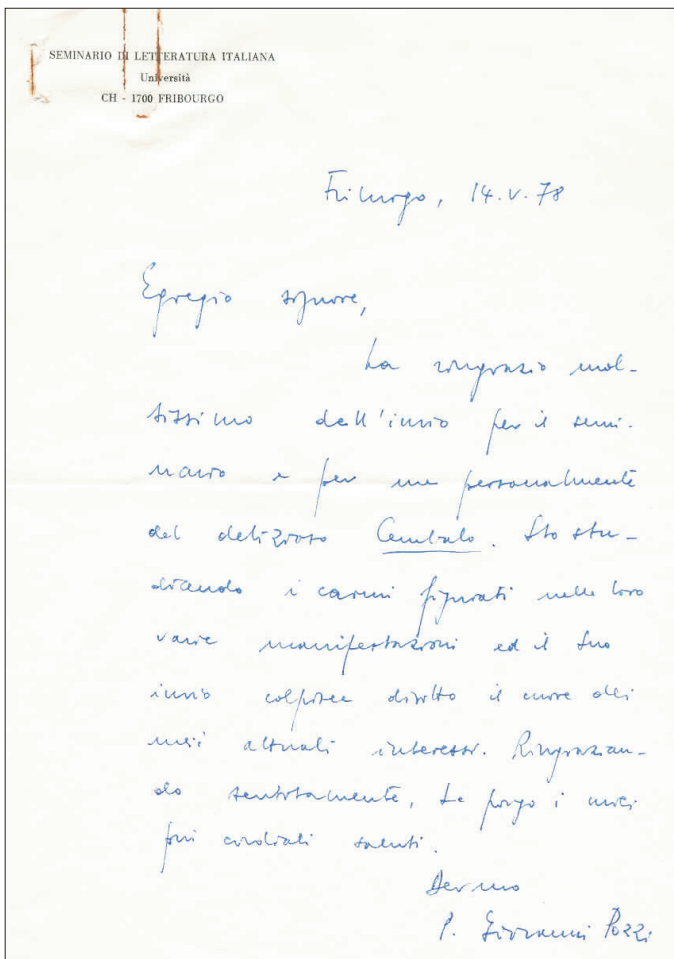



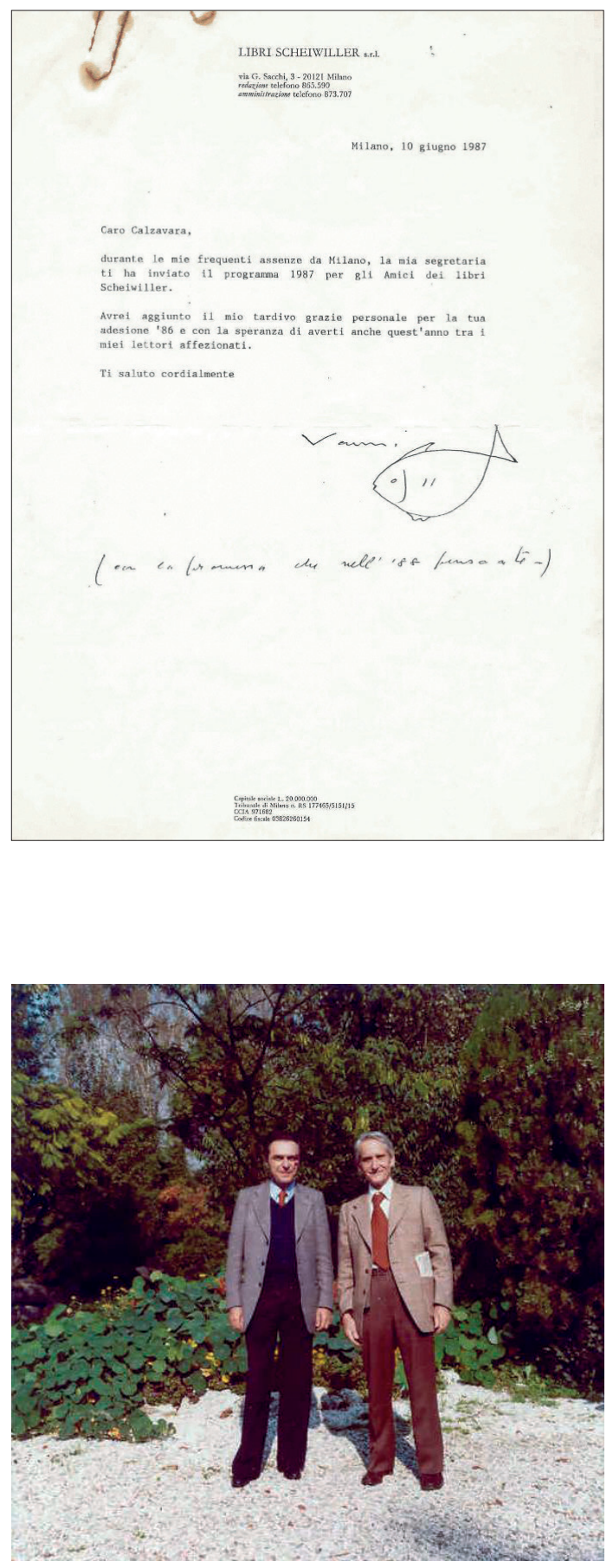

Figura 18

Lettera di Vanni Scheiwiller a Ernesto Calzavara. Milano, 10 giugno 1987
Figura 19

Calzavara scrive

sul retro della foto:

«Treviso, settembre 1978,

Ernesto Calzavara

in compagnia

di Cesare Segre» 


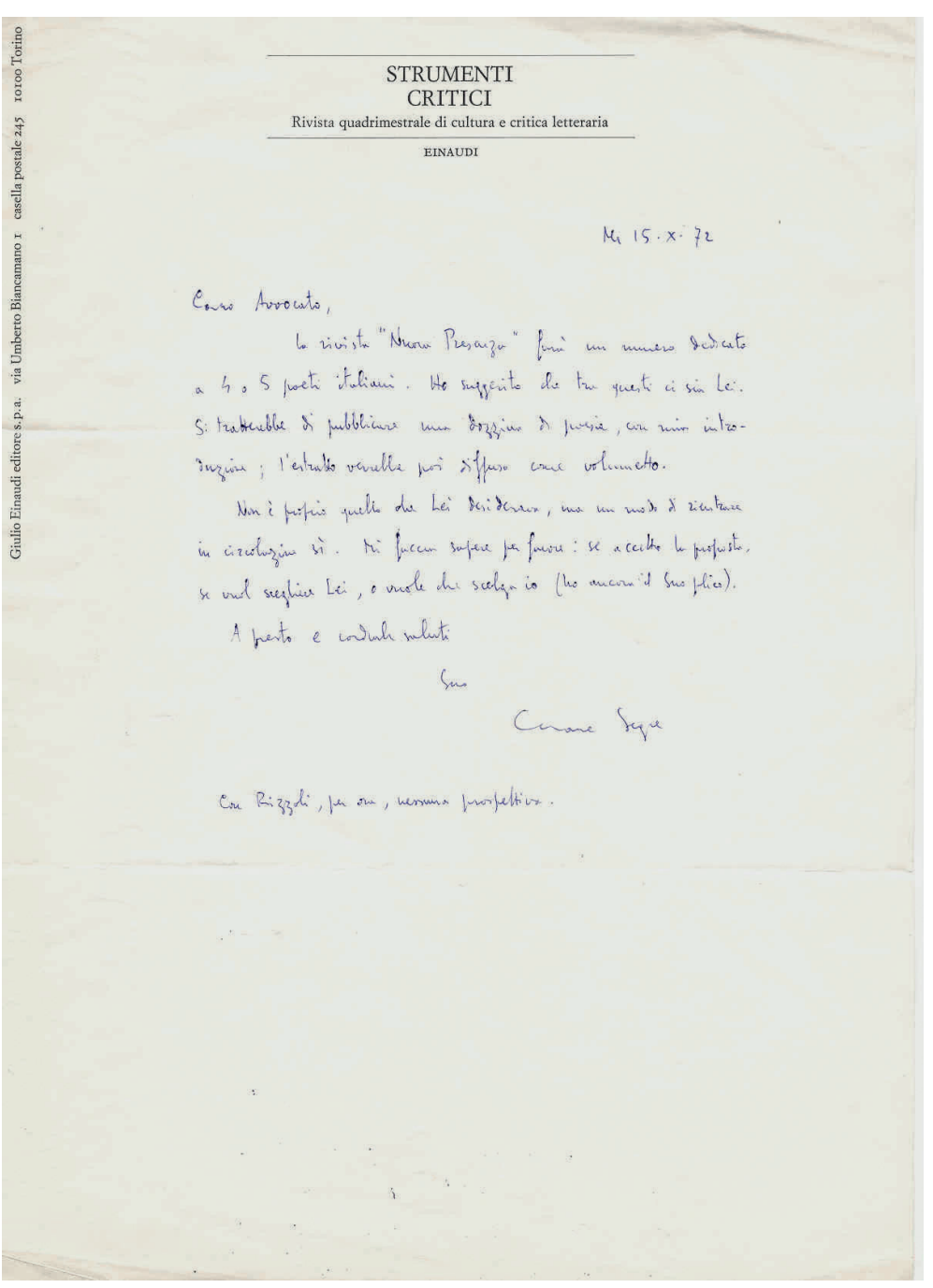

Figura 20 Lettera di Cesare Segre a Ernesto Calzavara. Milano, 15 ottobre 1972 


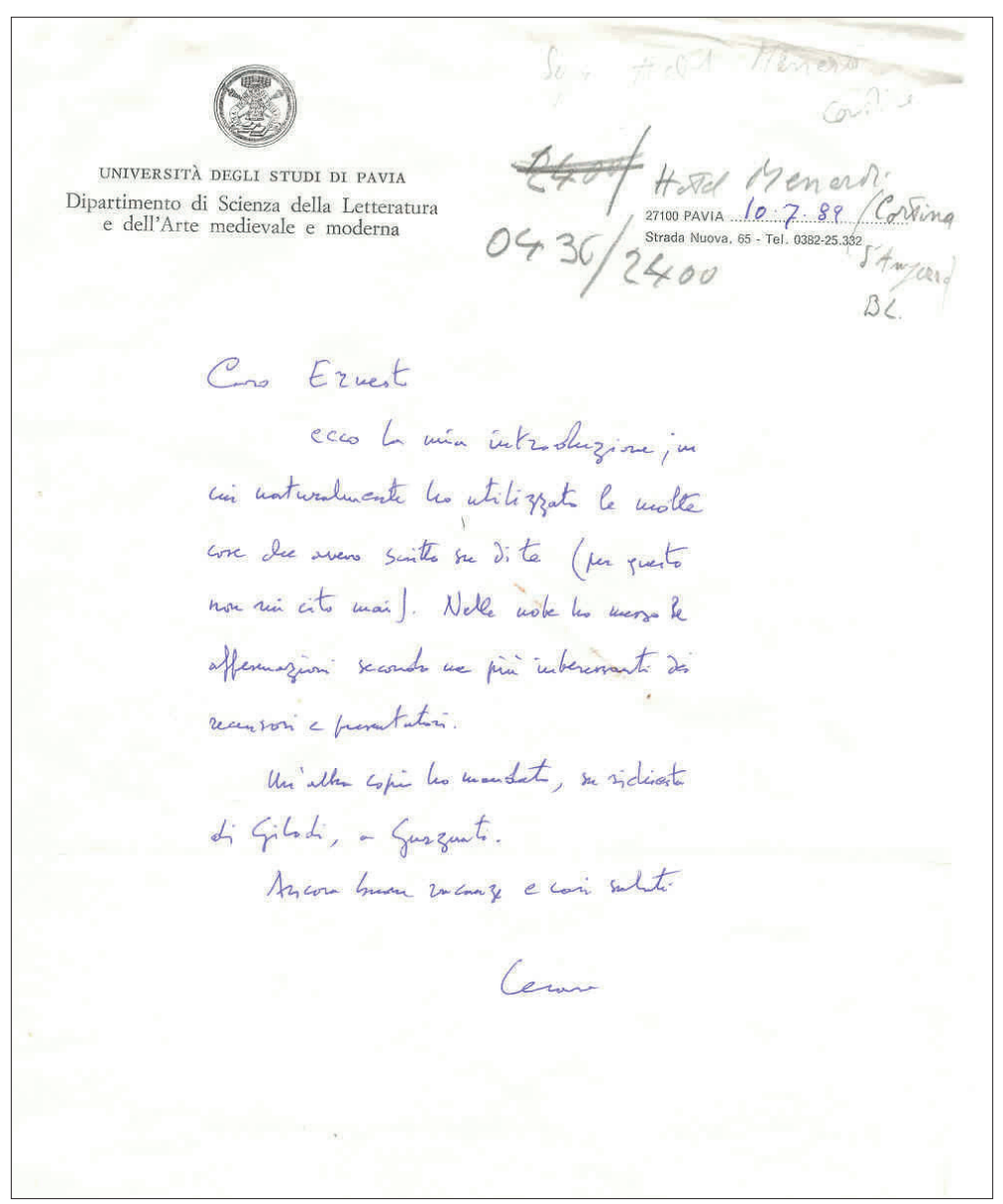

Figura 21 Lettera di Cesare Segre a Ernesto Calzavara. Pavia, 10 luglio 1989 
Anna Rinaldin

Rapporti culturali nella tradizione poetica del Novecento dalle lettere di Ernesto Calzavara

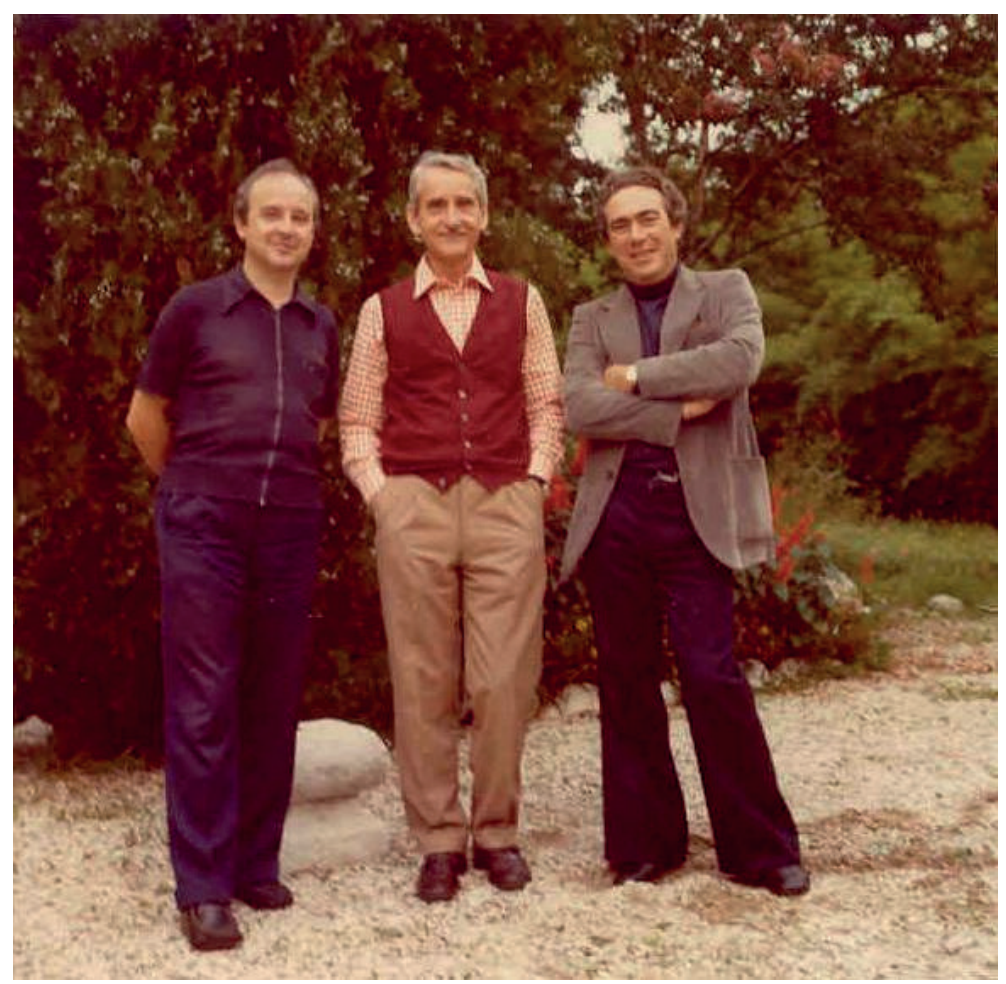

Figura 22 Calzavara scrive sul retro della foto: «TVIX-76 (o 78) Vanni Scheiwiller io Sandro Zanotto (manca la negativa un'altra copia l'ha Scheiwiller), 

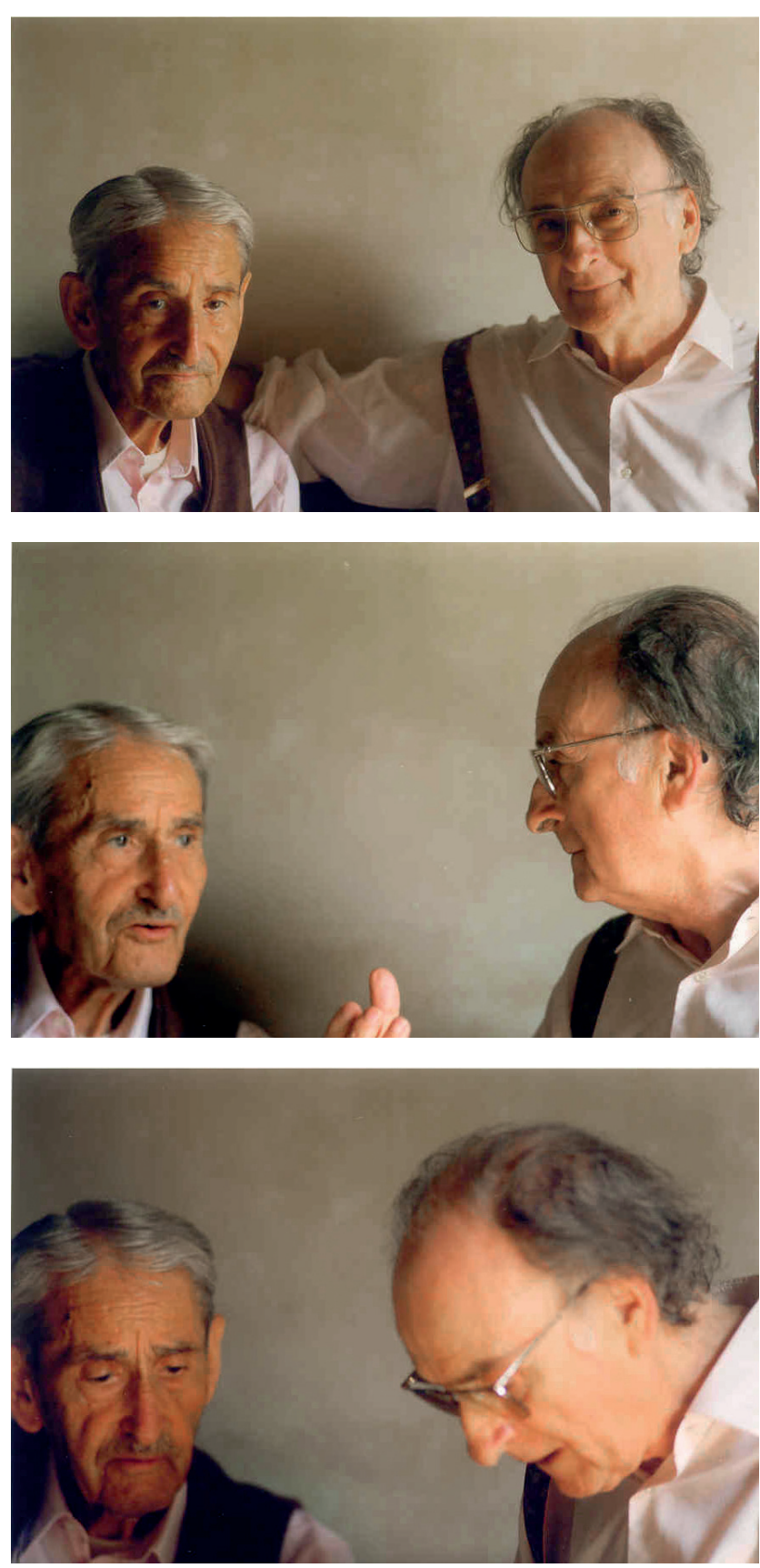

Figure 23-25 Ernesto Calzavara a Stra nel 1998 con Andrea Zanzotto (foto Pervinca Rizzo Calzavara) 


\title{
Omaggio ad Andrea Zanzotto Patrizia Valduga e Riccardo Held
}

\author{
Silvana Tamiozzo Goldmann \\ Università Ca' Foscari Venezia, Italia
}

Per il centenario della nascita di Andrea Zanzotto, ho pensato che un modo bello di rendergli omaggio fosse quello di chiedere una 'riflessione-lampo' a due poeti e traduttori, entrambi ben noti: l'intento è stato quello di ascoltare due voci che dessero una prospettiva inedita in mezzo ai numerosi interventi critici, alle molte iniziative che circondano questo grande poeta.

Patrizia Valduga è stata legata a Zanzotto da una profonda amicizia, una vera corrente di energia intellettuale testimoniata da numerosi incontri e da alcuni interventi in rivista: ${ }^{1}$ ha saputo illustrare

1 Tra gli interventi di Valduga ricordo: «A Zanzotto». l'immaginazione, 230, maggio 2007, 66 (numero monografico dedicato al poeta e da questi aperto con il breve scritto «I miei 85 anni»); «Per ricordare Andrea Zanzotto». finnegans, 21, 2012, 18-21; «Appunti sulla "comunione dei vivi e dei morti"». Zanzotto e Raboni, Hommage à Andrea Zanzotto $=$ Actes du colloque (Paris, les 25 et 26 octobre 2012). Cahiers de l'Hôtel de Galliffet, 2014, 65-70. Degli incontri e tavole rotonde mi limito a ricordare, tra i tanti (al-

$\begin{array}{lll} & \text { Peer review } & \\ & \text { Submitted } & 2021-07-12 \\ \text { Edizioni } & \text { Accepted } & 2021-07-26 \\ \text { Ca'Foscari } & \text { Published } & 2022-02-17 \\ & \text { Open access } & \end{array}$

(c) 2021 | (-) Creative Commons Attribution 4.0 International Public License 
con rara efficacia nelle diverse sedi (tavole rotonde, convegni, lezioni universitarie) la forza e l'incanto dei suoi testi poetici: la sequenza delle «nove schegge» è il dono che fa alla rivista di una storia di poesia davvero rara e preziosa.

Riccardo Held non ha 'viaggiato' con Zanzotto, l'ha piuttosto incrociato più volte sul suo sentiero e per lui ha organizzato nel 2005 un incontro veneziano non dimenticabile. ${ }^{2}$

In numerosi incontri in Italia e all'estero, Held mette da molti anni a disposizione del pubblico la sua memoria poetica (l'espressione francese par coeur è più calzante!). Anche in quell'occasione la sua capacità espressiva si era fatta riconoscere e amare. Le pennellate critiche del suo scritto ne sono una conferma che non abbisogna di chiose.

cuni all'Istituto Veneto di Scienze, Lettere ed Arti di Venezia), uno dei più belli, quello del 23 giugno 2010 all'Auditorium Comunale di Follina, visibile su YouTube: https:// www.youtube.com/watch?v=VZ3aGoeuMb8.

2 Mi riferisco alla Intervista in scena con Andrea Zanzotto organizzata e introdotta da Riccardo Held la sera del 16 giugno 2005 presso il chiostro del Telecom Italia Future Centre di Venezia nell'ambito della rassegna Bel rumore Estate. In dialogo con Zanzotto Francesco Carbognin, Renato Capoia e chi scrive in veste di moderatrice. Il testo, ricavato dalla registrazione video dell'evento da Francesco Carbognin, è entrato, come lo scritto di Valduga, in Hommage à Andrea Zanzotto = Actes du colloque (Paris, les 25 et 26 octobre 2012). Cahiers de l'Hôtel de Galliffet, 2014, 247-59. 


\title{
Schegge di nove lustri con Zanzotto
}

\author{
Patrizia Valduga
}

«non sta nel cammino esemplare

di un Soligo trapunto a piccoli cieli-meli-steli» ${ }^{3}$

Quando sono stata la prima volta a Pieve di Soligo? L'ho annotato nel quaderno del 1976: mercoledì 24 novembre, pomeriggio. Avevo fatto avere una lettera a Zanzotto tramite una sua ex allieva e ottenuto un appuntamento alla stazione di Conegliano. Arrivo in treno da Venezia: l'aspirante poetessa che non conosce la riconosce subito da lontano, e mi viene incontro. In auto verso Pieve dice che sono «cerea», «imbullonata»... E domanda a se stesso: «Ma perché non viene mai da me una giovane sana e allegra?». E io, un po' risentita: «E perché mai una giovane sana e allegra dovrebbe venire da lei?». Si volta verso di me, e mi guarda per qualche secondo: uno sguardo fiammeggiante, e profondo come un colpo di sonda, come una radiografia del mio cervello. Poi, con gli occhi di nuovo sulla strada: «Vero anca questo». In quel momento, io così piccola mi sono sentita accolta da lui così grande.

«o memoria con meco t'incammini,

lo sparso accordi e riconformi il fratto ${ }^{4}$

Ogni volta arrivo a Conegliano con un carico di angosce, che gli scarico addosso ogni volta. Tutti i problemi fisici, psichici, sessuali, affettivi mi vengono fuori come vengono fuori da uno psicoanalista. Che bene mi fanno, che bene mi fanno le sue parole: illuminazioni, vertigini, sprazzi di grande gioia... Mi dice di non fumare: «il fumo fa male, soprattutto a me»... E l'Ipersonetto del Galateo in bosco, quanto ci ho messo a impadronirmene, nutrirmene, assimilarlo? Pochissimo: nella primavera del '79 comincio a metter su la mia sonetteria come si mette su una baracca, una baracca abusiva della «metropoli Zanzotto». I materiali li prendo da lui, e li assemblo meglio che posso: è la mia seconda vita che comincia.

«Dimmi che cosa ho perduto

dimmi in che cosa mi sono perduto» ${ }^{5}$

3 Andrea Zanzotto, «Possibili prefazi o riprese o conclusioni», vv. 9-10 (La Beltà, 1968).

4 Andrea Zanzotto, «Ipersonetto (Sonetto dell'amoroso e del parassita)», vv. 5-6 (Galateo in bosco, 1978).

5 Andrea Zanzotto, «Collassare e pomerio», vv. 1-2 (Fosfeni, 1983). 
Nei quaderni degli anni Ottanta trovo: «La forma diventa contenuto: sembra il latte si fa formaggio». Andrea vuol dare le dimissioni dalla letteratura: «Son proprio stomegà». «Funzione ipnotica»: «settima, tra la conativa e la poetica». E negli anni Novanta: «Ieri sono uscito abbastanza felicemente senza beccarmi niente di nuovo». «Cioranetti»: Cioran + Ceronetti, epitome del catastrofismo sentenzioso. E i ricordi scivolano l'uno sopra l'altro... «Aria di fessura, aria di sepoltura», dico timidamente a chi guida in una giornata caldissima e abbassa un po' il finestrino; e lui, definitivamente: «Aria di finestra, colpo di balestra». Il finestrino viene chiuso all'istante.

«Per carità

esci dalla virtualità ${ }^{6}$

Gli dico al telefono che passo, cupa e ossessiva, intere giornate in internet per cercare un fidanzato. Esclama: «No, non devi!: lì ci sono solo i rimasugli». La parola «rimasugli» mi distoglie d'un colpo da quella occupazione disperata: l'ossessione svanisce, mi sento quasi allegra, e mi fidanzo persino, poco dopo, anche se per poco, molto poco; perché non viene mai meno il potere terapeutico delle sue parole.

«Nell'anno dei vermi

dei vermi dei vermi e dei vermi $\gg^{7}$

Zanzotto il mio ansiolitico, il mio antidepressivo? Anche, ma non soltanto. È soprattutto un distributore di energia psichica, e non soltanto per me, ma per tutti quanti, oltre che una potente medicina morale contro le ignavie e le infamie che infettano il nostro oggi. Sono fiera di aver bussato alla sua porta, sono piena di gratitudine perché l'ha aperta e mi ha fatto entrare. Oggi per stare con lui sto con i suoi versi, e quando sto con i suoi versi è come se lo pregassi: Andrea, affàcciati dal bordo del tuo cielo, rimetti in circolo il sangue della mia ultima vita.

«nessun tempo è mai passato

ogni tempo - unicamente - verrà ${ }^{8}$

6 Andrea Zanzotto, «Per carità», v. 1 (Conglomerati, 2009).

7 Andrea Zanzotto, «Silenzio dei mercatini 1», vv. 1-2 (Conglomerati, 2009).

8 Andrea Zanzotto, «Stanza immaginata o intravista», v. 8-9 (Idioma, 1986). 


\title{
Per Andrea Zanzotto
}

\author{
Riccardo Held
}

«Les lieux comme les dieux, sont nos rêves» ${ }^{9}$

Sono per natura diviso in due parti che non comunicano volentieri l'una con l'altra. Spero di avere sentimento quando scrivo poesia, ma quando cerco di capire, di analizzare poesia tendo alla razionalità e al sistema. Non mi è mai piaciuto parlare di un poeta da poeta, non l'ho mai fatto. Questa è la prima volta e mi obbliga a farlo la circostanza che da più di un anno vivo lontano dalla mia biblioteca, in cima a una collina che guarda Pieve di Soligo, e dunque queste brevi osservazioni sull'opera di Andrea Zanzotto le scrivo in tutti i sensi 'a memoria'.

\section{«Pace non trovo et non ò da far guerra» ${ }^{10}$}

Credo davvero, come alcuni studiosi e poeti, che la sua opera, da Dietro il paesaggio a Conglomerati, sia da intendersi come un unico grande libro. Il disegno esplicito nella sua prima raccolta, il dare avvio e consistenza alla rimozione del doppio trauma: guerra-guerre-sorelle, non si interrompe mai, varia e si amplia. Il rappresentato non è mai un problema in Zanzotto, pochi scriventi nel suo tempo hanno tanto robusta chiarezza di cosa deve essere detto e di come stanno le cose, il suo punto drammatico, come in ogni scrittore di razza, è quello di come si può dire, con quali suoni, con quali gomitoli, quali fonie si possa rendere inconfutabile quella certezza di com'era e com'è il mondo, innegabile per qualsiasi occhio che legga. Metterei in relazione due sequenze: 1) mondo dei significanti conciliati, forma governata buona, possibilità anche se parziale di riconoscersi in una tradizione, elementi del reale nominabili e davvero viventi, memoria non impedita dalla regressione, compassione; 2) mondo dei significanti inconciliati, il mondo della regressione psicologica e linguistica, la percezione non distinta e non governata, fantasmi che possiedono il paesaggio, la depressione, l'estraniazione. La prima sequenza si direbbe petrarchismo nel governo della materia formale, la seconda dantismo nella enormità della capienza lessicale, salvo che quest'ultima è ancora petrarchismo perché è una restrizione estrema alla rovescia, è un accumulo di tossine propiziatorie al compimento di quella contaminazione definitiva che è il fine del grande libro. Queste due serie si ritrovano nella sua poesia in combinazio-

9 Yves Bonnefoy, L'arrière-pays.

10 Francesco Petrarca, Canzoniere, CXXXIV, v. 1. 
ni differenti, quando esse toccano un punto di equilibrio si producono le sue poesie più grandi. Gli episodi di metricismo stretto, anche il più clamoroso, l'Ipersonetto di Galateo in bosco, che sono presenti in tutti i suoi libri, non sono affatto momenti di riposo dentro le certezze della tradizione, sono il suo metronomo, la sua catena di ancoraggio, sono il palo e la corda che dà al puledro la misura della sua libertà, lo lascia scorrazzare in giro.

Il mito fondante la sua scrittura ha a che fare con le sostanze sepolte, ma non con sostanze generiche e astratte, esse sono proprio lì sotto il suo paesaggio dal Montello al Piave. Il suo compito è doppio. Far riemergere, portare alla coscienza di tutti, elaborare e sedimentare il disastro di una guerra che il nostro paese sembra aver rimosso e poi conciliare quello che emerge con quel che ancora vive, con noi, con il suo paesaggio. Egli deve in altre parole compiere un doppio esorcismo. Il girovagare di Zanzotto per la lingua, dentro e sotto e intorno alla lingua è di tipo alchemico (l'autore del quale egli ha tradotto il maggior numero di pagine è il Balzac entusiasta di Svedenborg, egli stesso incontrerà i suoi seguaci in Svizzera), Zanzotto cerca la formula tra migliaia di accumulazioni di linguaggi e saperi. La formula alchemica ha anch'essa doppia funzione, quella di accelerare il processo di consunzione della sequenza-bosco-paesaggio-lingua-forma di vita associata moderna (il trionfo dell'oscuro) e nello stesso tempo cercare, esattamente come il ricercare dei provenzali, ma non la formula della perfezione amorosa, bensì un codice, una possibile scienza che aiuti la vita del dopo.

«den gib uns der die Wissenschaft gewinnt

das Leben aufzubinden in Spaliere

in welche zeitiger der Mai beginnt...» ${ }^{11}$

Quando sarà compiuta la consunzione, quando l'oscuro dell'orrendo massacro che ha fatto del Montello uno dei più grandi cimiteri a cielo aperto d'Europa avrà compiuto la sua opera di contaminazione, solo allora il bosco che si aggirava come uno spettro inconciliato tornerà a essere tale, un bosco vero, non un gorgo regressivo, e così la lingua, il paesaggio la forma umana.

«Mondo, sii, e buono

esisti buonamente». ${ }^{12}$

11 Rainer Maria Rilke, Das Stundenbuch, 1918, 87 («Dai quella a noi che scopra quella scienza | Di appoggiare la vita a dei tralicci | Dove maggio si affretti a cominciare»: trad. dell'Autrice).

12 Andrea Zanzotto, «Al mondo» (La Beltà, 1968, v. 1). 
Andrea Zanzotto è un poeta che pensa: un poeta che pensa con la mente e che pensa nel cuore. E tale pensare nel cuore egli compie dentro una forma. E questa forma, che dà cose, ne dà sempre una, ben definita, precisa, che si tratti della materia, che si tratti delle emozioni, del sentimento, o dell'immagine di una porzione del suo adorato, temuto, offeso paesaggio, sradicato e ritrapiantato in segni. È una sorta di diga di prevenzione per quanto ci viene incontro quotidianamente di svilito, di impreciso, di non commestibile e di non trasferibile entro la quotidiana esperienza del dolore e del piacere umano. La sua poesia è un girasole che il mondo e la storia han costretto a voltarsi verso l'ombra. 

Note e recensioni 



\section{Mario Isnenghi Vite vissute e no. I luoghi della mia memoria}

Francesca Bisutti

Università Ca' Foscari Venezia, Italia

Recensione di Isnenghi, M. (2021). Vite vissute e no. I luoghidella mia memoria. Bologna: il Mulino, 327 pp.

Vengo dal lavoro appassionante e impegnativo di leggere Vite vissute e no. I luoghi della mia memoria. Si tratta dichiaratamente di una autobiografia, cioè della ricerca di una autentica rappresentazione di sé, ma titolo e sottotitolo ci avvertono che c'è anche dell'altro: scoprire man mano che cos'è questo 'altro' è il valore aggiunto che offre la lettura dell'autoritratto di Mario Isnenghi. Nei 23 capitoli che compongono il libro ritroviamo lo storico, il docente, l'oratore, il poligrafo, il promotore di cultura, l'esempio animatore per tanti allievi, ma riconosciamo anche lo scrittore, l'artista della pagina che sa dare ai contenuti la forma che questi richiedono.

Intanto - è bene dirlo subito -, anche questo ultimo, a modo suo, è un libro di storia, dopo gli importanti studi storiografici - numerosissimi - che hanno dato fama al loro autore. Così scrive Isnenghi ricordando la pubblicazione del suo primo libro: «Non mi fermerò più. Avrò sempre un altro libro in fase di prima ideazione, mentre il precedente è in cantiere» (135).

Un'autobiografia è materialità dei fatti ma è, allo stesso tempo, interpretazione dei fatti e dunque luogo prediletto della soggettività. Il punto di vista di questo libro, sostenuto dall'inizio alla fine, è infatti

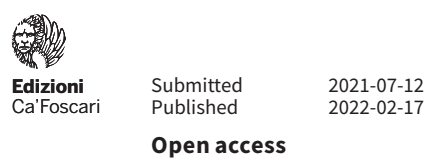

(C) 2022 | ()( Creative Commons Attribution 4.0 International Public License 
quello dell'io-Isnenghi che racconta in retrospezione i casi della propria vita: precisamente, la storia di sé dai 5 anni agli 82. Una storia personalissima che si intreccia però in modo organico e originale agli avvenimenti di quasi un ottantennio del nostro paese.

La narrazione inizia negli anni bui del dopo 8 settembre 1943, «in un'Italia fascista che si svincola da ciò che è stata e che cambia entrando in una terra di nessuno» (11), quando, scrive Isnenghi, «io sono piccolo, ma le cose che stanno accadendo sono grandi» (17). Il primo episodio che l'autore rammemora diventa un passo memorabile per noi che lo leggiamo oggi:

Una rottura primordiale, nell'infanzia. Svolta eterodiretta, decisionismo familiare che si riverbera su di me. [...] E le mie belle bretelle bianche incrociate di Figlio della lupa finiscono tagliuzzate e a brani nello scarico del water di servizio. I miei genitori avrebbero potuto farlo di nascosto, ma vogliono darci letteralmente un taglio e mi chiamano a vedere. Gesti sorprendenti e parlanti, senza spiegazioni: mi fanno assistere in silenzio. [...] Mentre mia madre traffica con forbici e sciacquone, cominciano i silenzi di mio padre, complice presente e inattivo. $(9,11)$

La madre agisce. Il padre tace. Lui, partito volontario, è reduce da una guerra perduta e per di più combattuta dalla parte sbagliata. Più avanti gli toglieranno il lavoro importante che ha alla Breda di Marghera: epurato. Il padre è figura tragica e i suoi silenzi, che sono i silenzi del vinto, tornano più volte durante la narrazione. Sono i momenti, molto intensi, in cui si ha la percezione che questa è anche un'autobiografia sentimentale, non solo intellettuale: «Ho sprazzi visivi di un viaggio di fortuna, soli io e papà - un'avventura iniziatica -, [...] su un camioncino, di quelli attrezzati all'epoca con panche e sedili» (35). C'è anche una «notturna locanda da romanzo» molto affollata dove i due sono venuti a riabbracciare il fratello del padre. Siamo nell'immediato dopoguerra e il bambino Isnenghi, che vede il padre guardarsi intorno e sente lo zio sussurrare la parola 'partigiani', «una parola che a casa è circondata di astio», ha paura. Forse anche lo zio è uno di loro? Del viaggio di ritorno, in treno, l'autore scrive solo delle lunghe gambe del padre, penzoloni fuori dal carro bestiame. E qui salta su dal profondo la memoria emotiva, quella che salda insieme passato e presente, quella che non si perde mai: «Nient'altro in mente: potremmo ancora essere lì in partenza da Milano, su quel carro, 'cavalli 8, uomini 40'» (36). Quel «potremmo ancora essere lì» è un fermo immagine, un'impressione che ferma il tempo, con l'accaduto che non smette mai di accadere di nuovo: una dichiarazione d'amore per il padre.

La madre, determinata custode del futuro della sua famiglia, ha già preso in mano la situazione e, all'arrivo degli occupanti a Venezia, visto che parla bene la lingua - è insegnante di inglese -, ha in- 
vitato a cena un ufficiale. Il padre «un po' subisce la disinvolta iniziativa diplomatica della moglie, ma lascia dignitosamente fare. Sta entrando, renitente, in una nuova fase di vita» (28).

L'Isnenghi bambino è parte inconsapevole di questa «comunità in scioglimento» (9). La sua naturale incapacità di comprendere i fatti enfatizza la complessità dell'epoca e il disorientamento generale: l'aereo che sembra puntare sul battello pieno di villeggianti/sfollati che lo sta portando a San Pietro in Volta è 'dei nostri' oppure è del nemico? Gli 'alleati' di cui la radio continua a parlare sono i buoni o sono i cattivi? Lui gioca sotto il tavolo del soggiorno e sente i discorsi dei grandi. Capisce e non capisce, come il Carlino Altoviti nella cucina di Fratta. ${ }^{1}$ Intanto, l'Isnenghi autobiografo ricuce per noi questi frammenti di memoria con il suo sapere storico e ci offre un racconto che è insieme testimonianza e documento.

Nel libro, la lente del punto di osservazione è mobile e molto duttile. Se la progressione del testo rimane cronologica e lineare, il fuoco dell'attenzione continuamente si restringe sul dettaglio e si amplia al contesto, mentre la memoria produce cortocircuiti del tempo e dello spazio, sospensioni, anticipazioni, retropensieri. Il lettore si ritrova così impegnato a seguire un percorso che si dà come rettilineo, ma che lo sorprende con le molte deviazioni, anche con curve che possono sembrare a gomito, come i passaggi, intorno ai 18-20 anni, nelle federazioni universitarie giovanili, dalla Fuci, dove «si fanno grandi letture, di saggistica e non solo di narrativa, si parla di concezioni del mondo, princìi, scelte» (69), alla Fgs, all’Ugi. Insomma dal cattolicesimo, via Socialismo, alla laicizzazione e all'impegno politico nell'area della sinistra. La transizione è lenta e meditata e nasce già all'interno della Fuci, dove «si sa ormai di dover 'aprire le finestre', leggere Gramsci» (72). Più avanti, la svolta pericolosa verso la zona grigia dell'Autonomia e dell'Operaismo non ci sarà per Isnenghi, che rimarrà un indipendente. Come scrive, «la figura dell'indipendente di sinistra è la mia, non ne ho mai avuta una di più adeguata» (245).

Nelle pagine programmatiche che aprono il libro, l'autore scrive di aver lavorato sull'io-me (la dimensione auto-riflessiva), forma sdoppiata di soggetto e complemento oggetto. Un'eresia grammaticale ma un accorgimento non solo retorico, assai fruttuoso, che permette altri sdoppiamenti: quello dell'io-noi (la dimensione collettiva) e quello dell' io-non io. Meglio sarebbe chiamarlo l'io no di Isnenghi: non sono mai stato democristiano; non sono mai stato comunista.

Il bello di questa scrittura è che ci ritroviamo, per via di associazioni e di indizi rivelatori, a passare senza apparente soluzione di con-

1 Nel 1968 Isnenghi curerà un'edizione delle Confessioni d'un Italiano per le scuole. Del romanzo di Nievo lo attrarranno «la grande narrazione, l'avventura ideologica, le dinamiche attivistiche della storia partecipata» (102). 
tinuità dalla storia di un individuo alla storia di tutti. Perché c'è un ulteriore pronome personale che si declina: il tu dei contatti e degli scambi con gli innumerevoli interlocutori (maestri e discepoli, colleghi professori e presidi, editori e politici, amici del cuore e amici del gruppo). Questi tu, così tanti e così diversi, li cogliamo come pluralità e il plurale di tu - come ha detto qualcuno - è, appunto, tutti.

Ma come si tiene insieme una struttura così complessa? Non certo solo secondo i modi di una catena logico-discorsiva, fatta esclusivamente di argomentazioni serrate, catena che sarebbe in ogni caso tanto forte quanto il più debole dei suoi anelli. Semmai, l'ordine del libro può essere assimilato a un tessuto, i cui fili, qui e là, si possono anche perdere, lasciando magari qualche smagliatura, qualche vuoto o qualche non detto. Ma è un tessuto che comunque tiene. Tiene alla prova della scrittura: grande ricchezza ed estrema cura del lessico, scansione della frase ora ampia ora sincopata, sorprendente capacità di stringere il concetto e di offrirne una sintesi espressiva. E dire, come dico, che è una questione di lingua e stile equivale a dire che è una questione di sostanza.

Non manca l'umorismo. Un quadro ritrae in poche righe il gruppo di artigiani e pittori anarchici che dipingono sapidi proverbi popolari su servizi in ceramica e «si muovono sempre tutti insieme nelle calli veneziane» (131). Nello stesso capitolo, dedicato agli anni belli e importanti dell'iniziazione all'insegnamento, due scene speculari sono puro teatro: gli scrutini a Chioggia, dove Isnenghi è professore incaricato, e un esame di stato a Nuoro, dove si trova a fare il commissario esterno. Giovane «illuminista e astratto», vuole voti secchi. Se è 4, sia 4 e non 6 meno. I due presidi, che conoscono «il contesto, le famiglie, i punti di partenza», vogliono negoziare. Intanto i comprimari (gli altri professori) «sfollano infastiditi a prender aria» nella prima scena o assistono silenziosi e imperturbabili nella seconda. Anche qui, come in tante pagine del libro, l'autoironia fa da sorvegliato contrappeso all'autoreferenzialità. E anche qui la vicenda personale diventa parte integrante del quadro generale: gli esami di stato «sono o non sono un meccanismo di unificazione del sistema culturale? O i rispetti territoriali relegano i giudici nazionali in un ruolo di pallida ratifica?» (137).

Ci sono momenti nei quali l'intimità ha la meglio sul distacco, per esempio quando l'autore scrive della genealogia degli Isnenghi/Dal $\mathrm{Ri}$, quasi una mitografia che ha per sfondo la Riva sul Garda delle sue estati di ragazzino, «quel tempo fondativo» (42) nella grande casa dove «vivi e morti [sono] ugualmente presenti» (30) perché la vecchia zia, grande affabulatrice e sua prima maestra di storia, non smette di raccontargli delle loro vicende. ${ }^{2}$ una vita a passo di carica, | adesso mi soffermo volentieri | sui sogni, sui dolori e le spe- 
I suoi maestri, per magistero diretto o indiretto, saranno poi Francesco De Sanctis, letto al Liceo Foscarini e poi riletto, il coltissimo don Germano Pattaro, guida sprirituale alla Fuci, Luigi Russo, penna esuberante e apripista degli studi culturali. Ma c'è stato anche Wladimiro Dorigo, modello di concretezza politica. L'eredità che Isnenghi ha ricevuto da loro è forse riassumibile in una frase che sta a metà del volume, quindi nel cuore di questa autobiografia: «Il problema è decifrare i segni dei tempi e la ricaduta dei grandi orizzonti nel 'qui e ora'» (153). Altrove scrive che è anche necessario fare il percorso inverso, studiare l'intrico dei piccoli fatti e da quelli risalire ai grandi rivolgimenti. Isnenghi, il decifratore di segni, è lo storico che conosciamo perché è, insieme, anche un letterato. Docente per vocazione, questa doppia appartenenza gli ha permesso di trasmettere ai suoi allievi un lascito importante, che, come ha scritto Silvana Tamiozzo Goldmann, rappresenta «una corrente di energia di pensiero che continuerà a scorrere negli anni». ${ }^{3}$

E gli allievi sono i protagonisti dell'ultima parte del libro, dove Isnenghi-professore, dopo i vent'anni di docenza a Ca' Foscari, fa i bilanci sulle loro diverse sorti accademiche. Ammessi o no ai concorsi. Vincitori o no. Esultanza oppure amarezza. Quando scrive di loro, gli scappano un po' di quelle effusioni che, come narratore, vorrebbe tenere a bada, ma che, come maestro, non riesce a controllare del tutto. Possiamo anche chiamarla affettività. Proprio a uno dei suoi eredi, Alessandro Casellato, devo la mia recente, ulteriore e diversamente consapevole rilettura dei Promessi Sposi. Un noto passo merita di essere ricordato qui, perché tematizza l'altro versante di competenza dello storico Isnenghi, la politica, per lui vitale quanto la letteratura:

Ma cos'è mai la storia [...] senza la politica? Una guida che cammina, cammina, con nessuno dietro che impari la strada, e per conseguenza butta via i suoi passi; come la politica senza la storia è uno che cammina senza guida. ${ }^{4}$

Certamente, quando per Isnenghi si è trattato di scegliere tra la politica e l'insegnamento, la decisione è stata netta e definitiva, presa già nei primi anni Sessanta. Così scrive di quando è giovane insegnante di Italiano e Storia tra Feltre, Venezia e Chioggia: «voglio fare quello che sto facendo, l'insegnante, voglio fare scuola (scuola,

ranze | di tutti i miei romantici antenati» (Bracco, G. Sull'orizzonte dei binari in fuga. Carme famigliare. Milano: La vita felice, 2000, 25).

3 Tamiozzo Goldmann, S. «Recensione di Vite vissute e no. I luoghi della mia memoria di Mario Isnenghi». L'immaginazione, 323(63-4), 2021, 63.

4 Manzoni, A. I promessi sposi. Commento critico di L. Russo. Firenze: La Nuova Italia, 1967, 516 . 
non università: l'università è venuta dopo, per conto suo)» (10). Una scelta professionale che è stata anche scelta di vita. Sposerà una sua ex-studentessa e con lei dividerà lunghissimi anni.

Dunque le vite non vissute del titolo sono quelle che l'autore ha scartato o scansato. Ma sono anche le vite che si sono accompagnate alla sua, parallele o solo tangenti, comprese quelle dei suoi allievi capaci che non hanno potuto trovare spazio professionale nell'università. Moltissime di queste ha saputo raccontarci, magari solo a sprazzi, perché uno scrittore sa anche vivere le vite che non sono state la sua.

La bella fotografia della copertina è di Laura Isnenghi, la figlia di Mario. Lo sguardo dall'alto non ci offre un panorama, ma la vista dello stretto rio di Ca' Pesaro, quasi uno squarcio tra le case che lo costeggiano. A lettura conclusa, l'immagine si rivela in tutta la sua forza dinamica: è un invito alla discesa, dai tetti pieni di luce all'acqua del canale in ombra, verso il recupero di qualcosa che sta giù in basso, per farlo riemergere dall'oscurità. Rappresenta il viaggio dell'autore che è andato a riscoprire per noi il suo percorso. Ė il metodo ed è l'insegnamento di questo libro: guardare oltre se stessi e dentro se stessi, con coraggio, fino in fondo. 


\section{Rivista annuale}

Dipartimento di Studi Umanistici Università Ca' Foscari Venezia

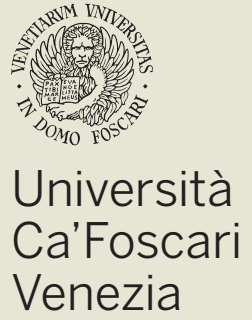

